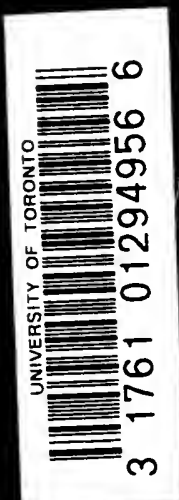

RGEMENT OF THE PROSTATE

C. W. MANSIEIJ MOULIIN 


$$
\begin{aligned}
& \text { Bought of } \\
& \text { BI,AKISTON'S SON \& CO.. } \\
& \text { MEDICAL BOOKSELLRR, } \\
& \text { No. 10I2 WaInut Street, } \\
& \text { PHILADELPHA. } \\
& \hline
\end{aligned}
$$

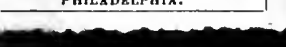




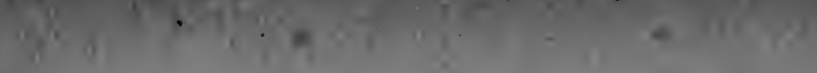


ENLARGEMENT OF THE PROSTATE. 
BY THE SAMF ALTHOR.

The Operative Treatment of Enlargement of the Prostate. (II unterian Lectures llelivered at the Royal College of Surgeons, 1:92). Royal svo, 5s. With fonr lingravings.

Sprains: their Consequences and Treatment. second Eitition, 1894. ('rown svis, ts. 6rl.

The Treatment of Sarcoma and Carcinoma by the Injection of Mixed' 'l'oxins. Deny 8vo, 3s. 6il. nett.

Inflammation of the Bladder and Urinary Feyer, $8 \mathrm{vo}, 5 \mathrm{~s}$.

When to Operate in Inflammation of the Appendix. Seconil Eitition, 1901. 2s. 6il. nett.

The Surgical Treatment of Uicer of the Stomach. 1902 , 2N. Gil. nett. 


\title{
ENLARGEMENT OF THE PROSTATE
}

ITS

\author{
TREATIENT AND RADICAL CURF
}

BY

C. MANSEll MOUllin, M.D. Oxon., F.R.C.S.

SENIOR SURGEON AND LECTURER ON SURGERY AT THE LONDON HOSPITAL; MEMBER OF THE COUNCIL OF THE ROYAL COLLEGE OF SURGEONS; EXAMINER IN SURGFRY IN THE UNIVERSITY OF CAMBRIDGF;

LATE RADCLIFFE'S TRAVELLING FELLOW, FELLOW OF PEMBROKE COLLEGE AND EXAMINER IN SURGERY IN THE UNIVERSITY OF OXFORD;

AND HUNTERIAN PROFEGSOR AT THE ROYAL COILEgE OF SURGEONS,

THIRD EDITION.

LONDON

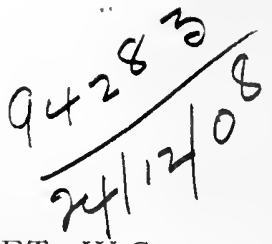

H. K. LEWIS, I36 GOWER STREET, W.C. 


$$
1+1
$$

PRINTED BY

H. R. LEWIS, I 36 GOWER STREET LON NuN, wac. 


\section{PREFACE TO THE THIRD EDITION.}

IN the five years that have elapsed since the last edition of this work was published, the surgical treatment of enlargement of the prostate has continued to make good progress. Wider experience has amply confirmed the opinions which I expressed then with regard to the relative value of the methods that were advocated; and now, I think, there are few who will fail to agree with me, that the pain and inflam. mation which used to occur in the course of this complaint with such frequency were often as much the result of the treatment as of the disease. It can no longer be doubted that enlargement of the prostate, in a very large proportion of instances, is perfectly capable of being cured, without undue risk, if only the consequences that follow from the indiscriminate use of catheters have not already inflicted irreparable injury upon the patient's bladder and kidneys.

\section{MANSELL MOULLIN.}

69 Wimpole Street, W. 



\section{PREFACE TO THE FIRST EDITION.}

Two years ago, in the Hunterian Lectures delivered at the Royal College of Surgeons, I pointed out that, in striking contrast to the great advance made by surgery in other directions, there had been but little progress of late in the treatment of enlargement of the prostate. Operation with a view to permanent cure was still regarded as upon its trial, and the catheter was looked upon as almost the sole means for obtaining relief.

Since then much has been accomplished. New methods have been devised. Wider experience has confirmed the opinion I expressed as to the merits of more active measures than those usually adopted. The old view as to the purely sexual character of the prostate, which had been almost forgotten and which I supported strongly, has gained ground again, and, in its turn, has led to the discovery of other methods; and now it is not too much to say that perfect relief can be assured even in the most advanced stages of the disorder. Further improvements no doubt will follow; but it has seemed to me that the time has come when that which has been done already might profitably be gathered together and recorded.

I am aware that in many respects the conclusions I have ventured to express differ materially from those still current. It will be thought, for example, that I have laid undue stress upon the evils that attend the habitual employment of catheters. But the very considerable opportunities I have enjoyed of studying the course of prostatic disease under the most varied conditions have left in my mind no doubt as to the comparative merits of the different methods that I have tried to describe impartially. 



\section{CONTENTS.}

\section{CHAPTER I.}

The Normal Structure and Function of the Prostate.

Size and Weight-Relations-General Structure-The Median LobeBlood Supply-Minute Anatomy-The Prostatic Urethra-The Veru Montanum-The Prostatic Utricle-Henle's Muscle-The Development of the Prostate-Its Function-The Caput Gallinaginis, and the Relation of the Prostate to the Sphincter of the Bladder Pages I-rg

\section{CHAPTER II.}

\section{The Enlarged Prostate.}

Histology-Rate of Growth-Prostatic Tumours-Physical CharactersVarieties of Enlargement-Influence upon the Urethra-Frequency with which Enlargement Occurs-Frequency with which the Different Portions are Enlarged . . . . . . Pages 20-33

\section{CHAPTER III.}

The Causes of Prostatic Enlargement.

The Time of Life at which the Enlargement Begins-The Analogy between Fibroid Tumours of the Uterus and Enlargement of the Prostate-The Connection between General Atheroma and Enlargement of the Prostate-The Theory of Compensative Hypertrophy-A Possible Solution in the Relation between the Prostate and the Testes. 


\section{CHAPTER IV.}

The Effects of Enlargement of the Prostate.

The Effect upon the Bladder-The Increase in the Amount of WorkCongestion of the Prostatic Plexus-Irritability of the Bladder-The Influence of Age, and of Cystitis-The Effect of the Enlargement upon the Kidneys . . . . . . . . Pages 50-65

\section{CHAPTER V.}

\section{The Symptoms of Prostatic Enlargement.}

Decrease in the Force of the Stream-Difficulty in Starting-Dribbling at the End-Intermittent Micturition-Residual Urine-OverflowInvoluntary Escape of Urine-Incontinence-Retention-Increased Frequency of Micturition-Impairment of Sexual Power-PainRectal Symptoms-Alteration in the Character of the Urine-Influence

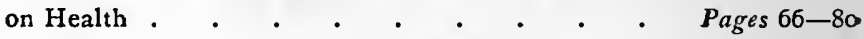

\section{CHAPTER VI.}

\section{Diagnosis.}

The Condition of the Prostate-Rectal Examination-Urethral Examination-The Length of the Prostatic Urethra-Its Direction-The Shape of the Orifice-The State of the Bladder-The Amount of Residual Urine-The Tone of the Muscular Coat-The Condition of the Mucous. Membrane-The Character of the Urine-The Condition of the Kidneys . . . . . . . . Pages 81-roo

\section{CHAPTER VII.}

The General Treatment of Enlargement of the Prostate. 


\section{CHAPTER VIII.}

The Local Treatment of Enlargement of the Prostate.

Palliative Measures: the Maintenance of the Urethra-The Prevention of Retention and of Residual Urine-The Prevention of Irritability of the Bladder and Cystitis . . . . . . Pages ro7-130

CHAPTER IX.

The Local Treatment of Enlargement of the Prostate.

Its Complication-Cystitis-Retention-Hæmaturia and Calculus

Pages 13I-15I

CHAPTER X.

The Radical Treatment of Enlargement of the Prostate. PArtial Prostatectomy.

Pages $\mathbf{5}_{52-175}$

\section{CHAPTER XI.}

The Effect upon Enlargement of the Prostate of Orchidectomy and of Operations upon the Structures contained in the Spermatic Cord

Pages 176-199 



\title{
ENLARGEMENT OF THE PROSTATE
}

ITS

\author{
TREATMENT AND RADICAL CURE.
}

\section{CHAPTER I.}

The Normal Structure and Function of the Prostate.

Size and Weight-Relations-General Structure-The Median LobeBlood Supply-Minute Anatomy-The Prostatic Urethra-The Veru Montanum-The Prostatic Utricle-Henle's Muscle-The Development of the Prostate-Its Function-The Caput Gallinaginis, and the Relation of the Prostate to the Sphincter of the Bladder.

ThE prostate is a glandular organ, containing a large amount of fibrous and unstriped muscular tissue. It is placed around the orifices of the ejaculatory ducts, behind and on both sides of the urethra. In function it is entirely sexual.

Its structure and relations are best studied in the young adult. At that time of life it has attained its full development, and has not yet undergone any pathological change.

In the fœtus the prostate consists of two separate parts, one belonging to each of the vasa deferentia. Before birth these join together across the middle line, forming what are known as the lateral lobes of the prostate. In the adult the shape of the gland is that of a truncated cone, placed with its apex downwards. The base is reniform in outline, convex in front, with, in the middle line behind, a depression as evidence of its mode of growth. Below the base the outline is more nearly circular, but the extent to which the separation of the lobes can be traced on the exterior varies greatly in different subjects and at different ages.

Size and weight.-The greatest length is four centimetres; breadth from 4 to 4.5 ; depth from 2 to 2.5 . The average 
weight is from 4.5 to 4.75 drachms (Thompson, "Diseases of the Prostate," 6th edit.).

Relations.-Above it, in the erect position, and resting upon the base, is the bladder, distinctly separate in the young subject. Below it is the deep layer of the triangular ligament of the urethra, with which its sheath is continuous. In front, at a distance of about half-an-inch, is the posterior margin of the symphysis pubis. On either side are the levatores prostatæ muscles, descending from the pubes, and, as it were, suspending the gland between them. Behind, entering the depression in the middle of the upper border, are the two ejaculatory ducts. These, with the vesiculæ seminales and the vasa deferentia, are held against the under surface of the bladder by a thin sheet of fascia, which, lower down, becomes continuous with the capsule of the gland. This sheet of fascia is all that separates the upper part of the prostate from the middle third of the rectum. Towards the apex of the gland the interval grows wider, the urethra inclining forwards, the rectum backwards.

The sheath that encloses the prostate is derived from the recto-vesical portion of the pelvic fascia. It is attached above to the posterior surface of the pubes and the sides of the pelvis. From this it descends to the groove that marks the junction of the bladder and prostate, except behind, where it does not reach lower than the posterior extremities of the vesiculæ seminales. At this level it divides into two. One layer passes upwards, and is lost in the wall of the bladder. This forms the floor of the pelvis, and shuts out from the proper pelvic cavity the prostate, the under surface of the base of the bladder, and the vesiculæ seminales. The other passes downwards, forms the sheath of the prostate, binds the vesiculæ seminales to the bladder, and is lost in the deep layer of the triangular ligament of the urethra. The prostatic plexus lies in the groove left by the divergence of the two layers, in the substance of the sheath outside the capsule of the gland.

Two parts of this fascia are distinguished from the rest as the anterior and lateral true ligaments of the bladder. They pass downwards and inwards from the posterior surface of the pubes and the sides of the pelvis respectively, and are 
somewhat stronger than the rest. Between the two anterior ones in the middle line is a narrow depression, formed by the fascia sinking down to be attached to the capsule of the gland near its apex, at its junction with the triangular ligament.

The urethra passes from above downwards, between the anterior margins of the lateral lobes. As it descends it curves forwards, so that the posterior surface of the gland is slightly longer than the anterior. The relative thickness of the tissues in front and behind the canal varies a good deal in different subjects. "It appears to me, judging from the conformation of the specimens, and not from the cut surfaces merely, that there is a rather larger mass of prostatic substance below (behind) than above (in front of) the urethra in all these specimens, except two or three "(Thompson). The bulk of the gland lies on either side, projecting outwards and backwards to form the lateral lobes.

The ejaculatory ducts enter side by side in the notch between the lateral lobes. They run forwards and slightly downwards, to enter the prostatic portion of the urethra a little below the middle. The lateral lobes, so far as their upper extremities are concerned, lie along the outer borders of these ducts. Below, they converge and meet upon the wall of the urethra. As they descend they fill up the open angle formed at the point of junction.

The anatomical relation of the prostate to the bladder.-As the circumference of the bladder diminishes towards the neck the longitudinal muscular bands collect into two main groups, one in front and one behind. These interlace to some extent with each other at the sides. Then changing their direction they sweep obliquely round, and are lost upon the outer surface of the prostate. The circular coat, if examined by making a vertical median section after the parts have been hardened in spirit, increases slightly in thickness at the neck. There is not, however, here anything that could be called a sphincter. Then it suddenly diminishes and is continued into the circular muscular coat of the urethra. In this the bundles, according to Griffiths, are more closely packed, and form a denser layer than in the bladder. The muscles of the ureters. which meet near the vesical orifice are prolonged 
down the urethra, chiefly along its posterior wall. Here they form an innermost longitudinal layer, interlacing behind and in the substance of the veru montanum, and lying almost immediately under the epithelium. The contraction of these fibres tends to shorten and dilate the urethra, and by bringing the surface of the trigone closer to the caput gallinaginis to level the angle between it and the bladder.

The lobes of the prostate do not belong to the urethra but to the ejaculatory ducts.

It occasionally happens when a kidney is deficient that the corresponding vas deferens fails to be developed. When this is the case the testes may be perfect, but the corresponding half of the prostate is small and ill-formed. This is a fact of very great significance. It shows that the dependence of the prostate upon the perfect development of the vasa deferentia is closer than its dependence upon that of the testes.

In many mammals, the lobes of the prostate are attached to the ejaculatory ducts upon their outer sides at some little distance from the bladder. In man there is no interval, and hence they appear to be interposed for a very short distance between the two chief muscular layers of the bladder. The longitudinal muscular coat, which has to a certain extent an independent origin from the pubo-prostatic and other ligaments, is lost upon their outer surface. The circular one is continued down between them in the wall of the urethra.

\section{Structure.}

The capsule.*-The prostate, except at its base and apex, is invested by a capsule of strong fibro-muscular tissue. From this trabeculæ pass into the interior, dividing and supporting the gland tubules. The prostatic plexus of veins surrounds the base in front and along the sides, lying in the substance of the sheath, outside the capsule. Over the lower part of the gland the capsule and the sheath are continuous with each other. Both are lost upon the deep layer of the triangular ligament.

* It has been suggested by Shattock that as the term capsule is mis. leading, and this layer is not definitely separate from the tissues beneath, it should be termed the cortex of the gland rather than the capsule. 
The secreting tissue of the prostate is collected chiefly into two masses. These are placed on each side of the urethra and the ejaculatory ducts, and form the lateral lobes. Usually there are a few isolated glands in the median line behind, with ducts that open into the urethra between the posterior end of the veru montanum and the vesical outlet. "The secretion may be seen oozing from these orifices when the median part of the prostate behind the veru montanum is compressed" (Griffiths, Four. of Anat. and Phys., 1888-9). More rarely a few are to be found in front as well.

The stroma, which consists of fibrous and muscular tissue, is distributed with greater uniformity. In front of the urethra it forms the whole of the prostate, and is known as the anterior commissure. Glandular tissue here is the exception. The closely-packed bundles of fibres extend transversely from side to side, and radiate outwards among the gland tubules of the lateral lobes. Forming the capsule or cortex, and immediately around the urethra, there is a thin uninterrupted layer, except that in the latter situation it is perforated here and there by the ducts.

Behind the urethra the arrangement varies at different levels. Near the apex the gland tissue of the opposite lobes may come into contact across the middle line. Higher up, just below the ejaculatory ducts, there is an interval, bridged across by stroma as in front-the posterior commissure. Higher still, between and above the ducts, there is in some instances a distinct little glandular mass, separate from the lobes on either side, but rarely large enough to form a projection between them.

The median lobe.-The existence of a median lobe as a normal structure is not admitted by Sir Henry Thompson. "I cannot find in healthy bodies below fifty years of age any formation in the situation described capable of being recognised as a distinct 'third' or 'middle lobe,' and am compelled to conclude that any marked prominence there which appears to possess independent characters (as regards size or form) must be considered abnormal or morbid." Sir H. Thompson, however, goes on to say that he considers it desirable to adopt for the upper segment of this posterior commissural part, a term which the French anatomists have employed, 
viz., "the median portion," on the ground that it bas a specific character which distinguishes it from other parts. "This character seems to be connected with that tendency to enlarge which this portion of the organ undoubtedly possesses. It is this, that the portion in question certainly contains a larger proportion of glandular structure than most other parts of the entire organ. Thin slices from this portion placed under the microscope and compared with slices from other parts demonstrate this very clearly." ("Diseases of the Prostate," 6th edit.).

I have repeatedly found tubules in this situation myself, both in the infant and in the adult.

Whether this part of the prostate lying between and above the ejaculatory ducts should be called the "median portion" or the "median lobe" seems to me to be immaterial. In many it is absent ; in a fair proportion the tubules are easily found; in a few they are so numerous and so large as to form a definite little mass, thicker in the middle than at its borders, where it joins the lateral lobes. It depends upon the extent to which the prostatic tubules have been displaced from their original site in the course of racial development. In some they all retain their primitive bilateral position; in others a greater or smaller number have been shifted towards the middle line. The pathological median lobe, the structure that projects so frequently into the vesical orifice, and is such a serious impediment to micturition, has nothing to do with the anatomical median portion. As I have shown in my "Hunterian Lectures on the Operative Treatment of Enlargement of the Prostate" in many instances, if not in all, it originates entirely independently of the anatomical " median portion," and bears a totally different relation to the circular muscular coat of the bladder.

Blood vessels. - The arterial supply of the prostate is not very large. One important branch comes off from the inferior vesical artery, and others, smaller ones, from the internal pudic directly and from its middle hæmorrhoidal branches. Occasionally there is an accessory pudic. Branches from these vessels pierce the capsule near the base and form a network around the secreting tubules.

The veins on the other hand, are naturally capacious, and 
in old people frequently attain an enormous size. In the sub-mucous layer of the urethra, chiefly along its posterior wall, there are large venous sinuses which communicate freely with the sub-mucous plexus around the neck of the bladder. Sometimes these form great varicose masses projecting under the mucous membrane, like the hæmorrhoidal veins in the neighbouring bowel. In the prostate itself there is an immense number of small thin-walled veins around and between the gland lobules. From these, short wide channels pass upwards and outwards through the capsule, to open directly into the plexus that surrounds it.

This plexus lies outside the capsule in the substance of the sheath of the gland. It is formed in front by the dorsal vein of the penis and some small vessels that come from the lowest part of the anterior surface of the bladder. As it passes back it divides, surrounding the base of the prostate at its junction with the neck of the bladder. Farther back the two halves unite again, between the under surface of the trigone and the vesiculæ seminales. Here they communicate with the veins from the anus and the peri-rectal tissues, and receive nearly the whole of those from the bladder. Mr. H. Fenwick (fournal Anat., xix., 1885) has shown that in the young subject all these veins are accurately fitted with valves, so that none of the blood from the rectum can enter them, and there can be no backward pressure in the vessels in the walls of the bladder. In later life, however, these veins, which even in the infant are of very considerable size, become more and more varicose, until at last they form an enormous plexus from which the valves have long since disappeared, and through which the blood circulates with extreme slowness. Congestion therefore is of common occurrence, and is often followed by thrombosis. It is the exception not to find phleboliths somewhere or other, and often long chains of them, around the prostate in elderly subjects. Normally the whole of the blood from this region returns through the radicles of the internal iliac, but in later life there is a free communication between this plexus and the rectal veins. 


\section{Minute Anatomy.}

The glands are of the compound tubular type. The ducts, forty or fifty in number, short and wide, converge from the lateral lobes to the floor of the prostatic sinus on each side of the veru montanum. Those forming the median portion open as already mentioned above this, between it and the vesical orifice. Many of them run some little distance downwards in the wall of the urethra before opening into it. In most places they have no wall of their own, but are mere channels in the stroma lined with low columnar epithelium. Here and there, where for instance they traverse the mucous membrane, they are surrounded by a thin layer of fibrous tissue, but in no part are there any muscular fibres, transverse or longitudinal, belonging to them. In the infant the ducts constitute the whole of the glandular part.

The secreting tubules appear in section as groups of rounded spaces lined with a single layer of tall columnar cells. These rest upon a basement membrane in which there are a few nuclei. Between the cells, at their attached ends, are, according to Langhaus, a number of small pear-shaped ones which Griffiths describes as ultimately becoming columnar. The spaces communicate with each other freely, and groups of them unite together to open into the commencement of the smaller ducts. These in their turn converge to form the main ones.

The stroma.-Unstriped muscular fibres of unusual size are grouped around the terminal alveoli. In many parts the interspaces appear to be completely filled by them, without their forming definite layers. In the young adult they are especially distinct, fusiform in shape, rather short and broad in proportion to their length, with large ovoid nuclei. Later in life they are largely replaced by fibrous tissue.

The rest of the stroma consists of firm and dense connective tissue, witl a few unstriped muscular fibres scattered through it. In the outer part of the gland they are so numerous as almost to form a distinct layer.

In front of the urethra is a great transverse bar of stroma. Behind, except at the apex of the gland, is another almost 
FIG. I.

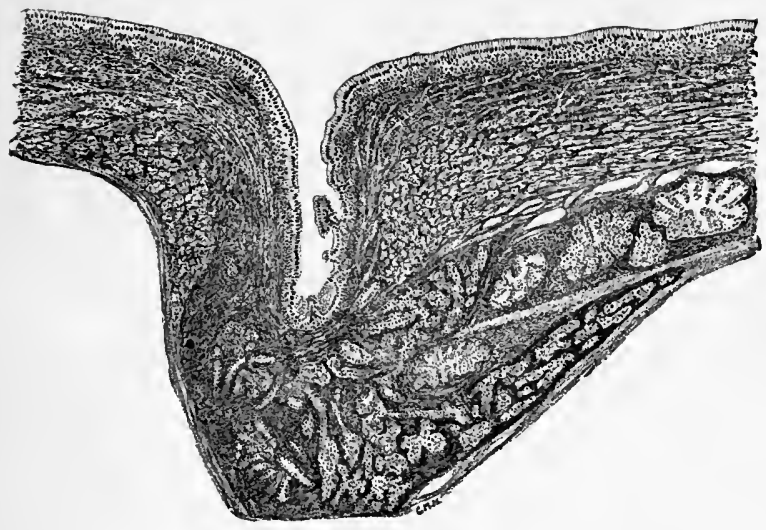

Sagittal section through the neck of the bladder from an infant six months old (a little to one side of the median line), showing the structural identity and continuity of the prostatic stroma with the tissue surrounding the vesiculæ seminales and the vasa deferentia. 

equally thick, but not so wide. From both of these branching strands radiate obliquely outwards, interlacing with the glandular tissue and meeting the prolongations inwards from the cortical layer.

The stroma of the prostate, like the glandular tissue it supports, belongs to the sexual organs and not the urinary system. In its histological features and in its origin from the genital cord, it resembles the tissue that surrounds and forms the wall of the vesiculæ seminales. If a sagittal section is made through the neck of the bladder and the prostate after they have been hardened in spirit, the stroma of the latter can be seen to be independent both of the circular muscular coat of the bladder and that of the urethra. In function it is entirely distinct. It does not aid in the expulsion of the urine. That, so far as the prostatic portion of the urethra is concerned, is carried out by the circular layer of muscular fibre immediately around it. It has to do solely with the discharge of the prostatic fluid, and as Dr. Griffiths (loc. cit.) has shown, its development during the rutting season in animals varies with that of the sexual organs. After castration it wastes as rapidly as the glandular tissue.

Dr. Griffiths regards it as merely part of the circular muscular fibre of the urethra. "At the level of the veru montanum, in the prostate of a fœtus of nine months, this circular coat is seen as a continuous sheet along the anterior wall of the urethra, whereas at the sides and posteriorly it spreads out in slender fasciculi, which invest the developing tubules of the prostate. It undergoes a special thickening in this region behind the veru montanum, where the developing tubules of the prostate grow out from the urethra, and the fasciculi derived from this part and accompanying the tubules in their divisions constitute the muscular element of the prostate. Thus the muscle fibres and the gland tubules grow on together, the former accompanying the latter in their whole extent, so that the muscle fibres form an investment to the gland tubules to their very extremities. The gland tubule growth, however, exceeds that of the muscle fibre, so that the glandular constituent forms a relatively and gradually increasing element of the organ. It is further to be observed that the ducts or excretory part of the prostatic 
gland tubules are devoid of a muscular investment. This results from the fact that these ducts occupy the situation near the veru montanum, between the mucous lining of the urethra and the circular coat, in which situation there is in the early fotal state, a considerable quantity of connective tissue. Hence the developing ducts traverse this tissue before reaching the circular muscular coat of the urethra and acquiring from it their muscular sheath." For the reasons above given, however, I am unable to assent to this view, and am compelled to regard the stroma of the gland as independent of the circular muscular layer of the urinary apparatus, and as much a part of the sexual system as the prostatic glands themselves.

The prostatic urethra extends from the vesical orifice to the triangular ligament, from three to three-and-a-half centimetres in length. Normally, its walls are in contact with each other (except when urine is passing down) and its shape on transverse section is crescentic with the convexity forwards. The internal diameter is about eight or nine millimetres, but it is capable of great distension without being torn or bruised.

The mucous membrane, which is covered with low columnar epithelium, is loose and soft, and contains a large amount of elastic tissue with numerous longitudinal muscular fibres. According to Dr. Griffiths there is a thin layer of circular muscular fibre inside this. Outside is a well-marked circular coat, continued down from that of the bladder.

The veru montanum.--In the middle line running downwards from the vesical orifice along the posterior wall of the prostatic urethra is an elevation, the veru montanum. It begins very gradually just below the uvula vesicæ, descends for a centimetre and a half or two centimetres, rising slowly and then suddenly widens out and comes to an abrupt termination. On each side of it is a depression, the prostatic sinus, on the floor of which are the orifices of most of the prostatic ducts. At its termination in the median line is a narrow slit, the opening of the prostatic utricle, and on the margins of this one on each side, are those belonging to the ejaculatory ducts. The mucous membrane over it is peculiarly soft, with large thin walled venous spaces in it, and is said to contain 
erectile tissue. Underneath are a great number of decussating muscular fibres, prolonged from the longitudinal muscular layer of the urethra.

The prostatic utvicle, the homologue of the uterus and vagina in the female, is a minute sac lying between the two lobes of the prostate, and opening into the prostatic urethra by a small orifice at the inferior end of the veru montanum. It is developed separately from the prostate, and is merely included in it in the process of growth. The cavity is lined with a single layer of columnar cells (said by some to be ciliated), resting upon a layer of loose connective tissue, in which are numerous venous spaces. Outside are some scattered bundles of smooth muscular fibre running transversely. The ejaculatory ducts lie on each side of it, closely adherent to it, and open close to it in the prostatic urethra.

Henle's muscle.-In transverse sections of the prostate about the middle of the gland a few striped muscular fibres are usually to be seen running across the front. Lower down these become thicker and longer and form what is known as Henle's muscle, or the external sphincter of the bladder. At the apex of the prostate there is a very distinct layer surrounding the front and sides of the urethra immediately outside the unstriped circular layer; just below this it passes imperceptibly into the constrictor urethræ.

\section{Development.}

The development of the prostate is of importance owing to the comparison that has been instituted between enlargement of the prostate on the one hand and fibroid disease of the uterus on the other.

The bladder and the first portion of the urethra, as distinguished from the prostate that surrounds it, are developed from the allantois, absolutely distinct from the rest of the genito-urinary passages. In the Monotremata they remain distinct; the penile part of the urethra, which is formed by the growth of the genital eminence on the anterior wall of the cloaca, in them never becomes organically united to the rest, or serves, as it does in the males of the higher order of mam-. 
malia, for the transmission of urine. As is the case with the corresponding organ in the female, it retains throughout life its purely sexual character. The same thing is sometimes seen in man, in cases where development has been arrested. In extreme forms of hypospadias, for example, the urethra opens in the perineum, and the penile groove is never closed in.

The Wolffian ducts become the vasa deferentia and ejaculatory ducts in the male, the ducts of Gärtner in the female. They open at first into the cloaca; later into that part of it which is marked off from the rest as the urogenital sinus. In some animals the ducts of Gärtner can be traced down the wall of the uterus and the vagina as far as the opening of the latter into the corresponding space, the vestibule.

Müller's ducts form the prostatic utricle in the male, the uterus and vagina in the female. As Bland Sutton has shown, the utricle corresponds to both uterus and vagina, and its orifice into the prostatic portion of the urethra to the orifice of the vagina into the vestibule.

The prostatic glands are described as originating from the mucous membrane that lines the urethra. If this were the case, they would be developed either from the allantois or from the urogenital sinus, which forms a small portion of the wall of the urethra below the orifice of the utricle. In other words, they would be developed from the urinary organs, and have no connection with the vasa deferentia or any other part of the sexual system.

The difficulty is apparent only. The explanation is to be found in the shifting of the site of origin in the course of racial development. It has gradually been transferred from the lower end of the Wolffian ducts to the structure into which the ducts open.

In many mammals the prostate retains its connection with the Wolffian ducts. It is attached to the distal ends of the vasa deferentia and consists of two separate glands. In the human fotus the lobes are still distinct, they lie behind the urethra, and even at birth their connection with it is much less intimate than it is in later life. Other organs change their point of origin in the same way. "The ureters, which are originally prolonged from and open into the Wolffian 
ducts, subsequently become shifted in position, so as eventually to open into the enlargement of the allantoic stalk from which the bladder is formed" (Schäfer). If reliance is to be placed upon the evidence of comparative anatomy there can be no doubt that the glandular part of the prostate has become shifted with the ureters a shorter distance in the same direction.

As I have already mentioned, if one of the vasa deferentia fails to be developed, the testis may be perfect, but the corresponding half of the prostate is small and ill-formed.

The stroma of the prostate is developed from the genital cord, a name given to the thickened mass of tissue that surrounds the Wolffian ducts as they course together to the cloaca behind the stalk of the allantois. The formation of this cord, according to Schäfer, is connected with the separation of the cloaca into a dorsal or anal, and a ventral or urogenital part.

The uterus, therefore, is homologous with part of the prostatic utricle and in no way with the prostate itself. The representatives of the prostatic glands in the female are to be sought near the orifice of the vagina, where it opens into the vestibule, around the ends of the ducts of Gärtner, and that of the stroma in the same way, in the substance of the perineum. So far as the evidence derived from development and comparative anatomy is concerned, the homology that has been supposed to exist between the prostate and the uterus is based upon error.

\section{The Function of the Prostate.}

The function of the prostate is entirely sexual. It assists the passage of urine by the mechanical support it gives to the urethra, and that this is of value is shown by the diminished thickness of the circular muscular layer in the middle of the prostatic portion. It does not take any other share in micturition.

The thin layer of longitudinal muscular fibres, lying immediately under the mucous membrane of the first part of the urethra, continued downwards from the muscles of the ureters, 
may help in micturition by tending to level the surface of the trigone and obliterate the angle between it and the urethra, but these fibres do not belong to the prostate.

If castration is performed in early life neither the glandular nor the muscular part of the prostate is developed.' If later, the prostate atrophies, and nothing is left but a small and hard fibrous nodule. In neither case is micturition interfered with in any way.

Dr. Griffiths (loc. cit.) has shown that, as with other sexual organs in animals, the size and perfection of the prostate (again both glandular and muscular parts) rise and fall with the breeding season.

It has been said that the function of the prostate is to act as a funnel-shaped muscular support to the bladder. This can only be true so long as the body is upright, yet the gland is proportionately as large and as well developed in animals that maintain babitually the quadrupedal position.

Moreover in children, in whom the prostate is not developed, and in women, the absence of this support never gives rise to any difficulty. This view, therefore, is quite untenable.

The secretion of the prostate, according to Fürbringer (Berlin Klin. Woch., July I9, I886), is a thin, slightly milky, acid fluid containing an emulsion (not of fat, but of lecithin; Posner) suspended in an albuminous medium. It is discharged into the prostatic urethra with the semen. If it is wanting, the semen fails in its characteristic odour; no Böttcher's crystals can be developed from it, and the spermatozoa, though living, are motionless.

Fürbringer arrived at the conclusion that its function was to arouse and maintain the slumbering vitality of the spermatozoa. He obtained the secretion of the testes, mixed with that of the vesiculæ seminales, from a patient who was under his care suffering from atonic spermatorrhœa. The semen that escaped passively during micturition or defæcation was entirely deficient in the qualities above mentioned. That, on the other hand, which was discharged after sexual excitement was normal. The difference he attributes to the absence in the former case of the prostatic secretion.

Further experiments made with the fluid yielded in his 
hands corroborative results. Small quantities excited to action motionless spermatozoa; larger ones killed them, probably from excess of acidity.

A peculiar structure, described by Lataste ( $A m n$. de la Sociêté de Biologie, Paris, I886) as the "bouchon vaginale," is found in the vagina of certain rodents after copulation. It is a gelatinous body, moulded to the interior of the cavity in which it lies, and secreted, except perhaps so far as its external layer is concerned, by the accessory male organs of generation. It liquefies on exposure to air and coagulates and becomes opaque on the addition of water. It is worthy of note that semen, after normal ejaculation, reacts in the same way; when it escapes in atonic spermatorrhœa, and therefore, is probably not mixed to any extent with the prostatic secretion, it is diffluent from the first.

The terminal secreting tubules of the prostate in all men over fifty years of age contain minute spheroidal or (where several are in contact) polyhedral bodies, concerning the nature of which there is a certain amount of doubt. They exhibit a concentric marking, as if formed by deposition upon a central nucleus in successive layers, and consist of an organic substance mixed with a variable amount of carbonate and phosphate (bone phosphate) of lime. When small, they are yellow, almost translucent, and strongly refract light. The older ones are darker and more opaque. According to Sir H. Thompson, the proportion of inorganic matter varies from $4^{6}$ per cent. in the smaller to 86 per cent. in the larger ones. In one case under my care, in which they were especially large, the percentage was higher still. The nucleus may be formed either of detached epithelial cells or from the secretion, which contains a mucoid or nucleo-albuminous substance and a considerable quantity of phosphate. The subsequent growth is undoubtedly due to the deposition round the central body of material secreted by the cells. From the extreme frequency with which these bodies occur, with or without enlargement of the gland, they may be regarded almost as one of its normal constituents.

The stroma of the prostate has to effect the discharge of the secretion from the gland tubules into the urethra, where it mixes with the fluid from the testes and the other sexual 
glands. "The secretion is retained in the tubules where it is being formed, but especially is it retained in the parts of the tubules nearest to the urethra, which are usually larger. Therefore there exists in this gland the simplest and, at the same time, the most primitive means for retaining its secretion until a demand for its expulsion into the urethra arises. The secretion thus accumulated is expelled into the urethra during the sexual act by means of the contraction, which begins at the distal end of the tube, of the complete and continuous sheath of non-striped muscle that surrounds each tubule in its whole length " (Griffiths, loc. cit.).

Secretion is in all probability a more or less continuous act throughout adult life. The discharge, at least of any amount, can only take place at long and irregular intervals. A considerable quantity must, therefore, accumulate in the tubules and the short, but wide, ducts, and the function of the peculiarly arranged museular fibre is to secure the expulsion of the whole of this practically at the same instant.

From an observation of the late Mr. W. Anderson, it may also be part of the work of the muscular fibre of the prostate to prevent the regurgitation of semen into the bladder. This sometimes occurs in cases of chronic inflammation of the prostate, owing in all probability to enfeebled action of the muscular fibres in the upper part of the gland near the neck of the bladder.

Finger, who describes the ejaculatory ducts as surrounded through their whole length by a layer of circular muscular fibre, regards the prostate as a kind of sphincter for them and for the vesiculæ seminales, in addition to its other duties. In chronic prostatitis the muscular fibre fails, and true spermatorrhœa occurs, the seminal fluid escaping into the prostatic urethra whenever there is an increase in the abdominal pressure, as in micturition or defæcation (Medical Fortnightly, April, 1893).

The caput gallinaginis. - The function of this structure, which contains a certain amount of erectile tissue, is said to be to cut off the urethra from the bladder during coitus. There is no doubt that it is functionally connected with the generative act, more especially with ejaculation. Irritation or inflammation of this part of the prostatic urethra leads to priapism 
and precipitate emission. Atrophy and induration cause impotence, with absence or considerable diminution of sexual sensation, and in extreme cases complete failure of ejaculation" (Finger, loc. cit.). It may, therefore, serve to prevent regurgitation (though this is with greater probability effected by the contraction of the muscular fibre in the base of the prostate and around the neck of the bladder); but it certainly has other duties. In connection with this it is worthy of note that it occupies the situation, and may, therefore, be the modified representative of the male organ for intromission in many of those animals which, like the Urodelous amphibians, retain the primitive cloacal outlet, such as exists in the human fœetus at an early period of life.

The relation of the prostate to the sphincter of the bladder.Guthrie ("Anatomy and Diseases of the Urinary Organs") long since pointed out that the sphincter of the bladder is at the end of the prostatic portion of the urethra, not at the so-called neck of the bladder. Fluid that enters the urethra anterior to the constrictor urethræ escapes by the penis; that which enters posteriorly, when it has filled the prostatic urethra, regurgitates into the bladder.

The bladder, when empty, is flattened from above downwards; the walls are in contact, and the cavity is obliterated. As urine enters, little by little the bladder expands, the muscular coat keeping up a slight tonic contraction on the contents. When a certain point is reached the tonic pressure of the muscle distributed over the body of the bladder overcomes that of the muscle around its neck, and the elastic resistance there as well. If the bladder is healthy this does not occur until it is distended; if it is inflamed, or the urine is irritating, it may occur with only a few drops. In infants micturition follows immediately as a reflex act. In adults, Henle's muscle and the constrictor urethræ, which being voluntary muscles, the infant has not yet learned to use, are called into play.

$\mathrm{Up}$ to this point there is a close parallel between the mechanisms of micturition and defæcation. Here the parallel ceases. The unstriped muscular coat of the bowel is stronger than the voluntary sphincter, and if the stimulus continues can overcome it. In the bladder, on the other 
hand, the muscular coat is the weaker, and becomes tired out first. Later, however, when the elastic tension of the wall is called into play by ever-increasing distension, the sphincter gives way and allows the urine to escape, drop by drop.

So long as the bladder contains only so much urine as it can accommodate without exciting the tonic contraction of its muscular coat, the internal orifice is closed. When this point is overstepped urine enters the prostatic part of the urethra, and this becomes physiologically (as it is embryologically) part of the bladder. "If we introduce an elastic catheter into the urethra of a man who has as yet no desire to urinate, until the urine begins to escape, and measure the portion of the catheter thus inserted, and then do the same thing with the same subject, when the bladder is full and desire is present, we always find that in the latter case the length of catheter necessary is from two to three $\mathrm{cm}$. shorter, and that as a matter of fact the urethra is so much shorter with a full bladder. Repeated experiments, carried out on healthy persons, showed that with a moderately full bladder the urethra was eighteen to twenty-one $\mathrm{cm}$. long; with a full bladder and a desire to urinate it was sixteen to nineteen" (Finger, Med. Fort., Chicago, I893).

Confirmative evidence may be obtained both in animals and in man by injecting plaster of Paris through the ureter into the empty bladder post-mortem. If only a small quantity is injected under light pressure the vesical orifice remains closed, and the cast is egg-shaped. If the quantity is larger and the pressure greater, the plaster forces its way into the prostatic part of the urethra, and the cast becomes pearshaped, the conical part representing the first portion of the urethra. dilated and physiologically forming part of the bladder.

Probably much of the discrepancy of opinion that exists with regard to the existence of a sphincter has arisen from the different condition of the bladders that have been examined. It is undoubtedly true that, as a result of continued irritation, the circular muscular coat may become so hypertrophied as to form a kind of sphincter; and in cases of enlargement of the prostate in which there has been much 
spasm for some length of time an immense degree of thickening, most marked around the neck, is not infrequent; but nothing deserving the name of a sphincter is to be found in this situation in the healthy bladder of a young adult. 


\section{CHAPTER II.}

\section{The Enlarged Prostate.}

Histology-Rate of Growth-Prostatic Tumours-Physical CharactersVarieties of Enlargement-Influence upon the Urethra-Frequency with which Enlargement Occurs-Frequency with which the Different Portions are Enlarged.

By enlargement of the prostate is understood a more or less uniform increase in size which not unfrequently makes its appearance as age advances, and which is in many instances associated with grave impairment of the function of the bladder.

It is not inflammatory. The course that it pursues, the symptoms to which it gives rise, and the histological changes it undergoes are entirely different from those that occur in inflammation.

Nor is it mere hypertrophy, compensatory or otherwise. The structure and arrangement of the tissues present material differences even in the earliest stages.

It is essentially a fibro-adenomatous growth, resembling in some measure the normal structure of the gland, not spreading through the sheath, but extending upwards in the mucous and sub-mucous coats of the urethra and bladder.

Histology.-So far as I have been able to ascertain, in the specimens that $I$ have examined, the primary change is the growth of imperfectly formed glandular tissue, recalling the tubular glands of the prostate, but without ducts and without arrangement. It may spread from the lateral lobes, causing them to increase enormously in size, or from the median, or from all three together. Or it may make its first appearance separately from any of these, in the sub-mucous layer of the urethra, originating, in all probability, in some of the small detached glands. Whatever its source it spreads upwards, towards the bladder, for in this direction there is least resistance. When the growth is small it may remain outside the circular muscular layer, merely raising the floor of the bladder from below. As it increases in size it nearly always 
grows through this layer and projects into the cavity of the bladder, covered over only by its mucous membrane.

The primary change is the invasion of the surrounding tissue by this adenomatous growth, which pushes everything to one side. The new tubules, for the most part, have an open lumen, and are lined with a single layer of low columnar cells, resting upon a basement membrane. Occasionally there is a tendency to the formation of acini, and sometimes these are so large that they deserve to be called cysts. Nowhere is there the orderly arrangement characteristic of the normal gland. The rate of increase may be rapid and irregular, leading to the formation of outlying bosses and nodules, perhaps far away from the parent mass and almost isolated from it, or it may be slow and uniform, so that the gland enlarges equally in all its dimensions. Wherever I could find the beginning it has always been the same.

Except in the region of the cortex, the stroma at first takes but a small share in the enlargement. There are merely a few long fibre cells, with large oval nuclei, arranged concentrically around the imperfectly formed tubules. In the cortical layer, however, the fibro-muscular tissue, from the very first, continues to increase in thickness, until at last it forms a kind of adventitious capsule surrounding the gland. Some of this increase is probably apparent only, caused by the eccentric pressure of the rapidly growing adenomatous tissue, which flattens all the structures that lie between it and the sheath, until they are spread around it in layers; but in most instances there is a definite growth of stroma tissue as well.

In certain parts, around the lower portion of the prostatic urethra, for example, the glandular arrangement is always less conspicuous than it is elsewhere. The growth often appears to be made up of nothing but a dense fibrous stroma. Even the long fibre cells, with large ovoid nuclei that are usually present, are not to be seen. Sometimes there are a few groups of epithelial cells lying in clefts and interspaces. More often there is only some granular débris to show where they once were. Occasionally this production of fibrous tissue begins at a comparatively early stage of the enlargement, and is not limited to the region of the urethra and cortex, but involves the whole, almost from the first, so that the gland becomes exceedingly hard and tough. 
The physical characters of the enlarged prostate depend upon the rate of growth, and the relative proportion of the fibrous and glandular elements. If the increase is rapid, and the glandular structure predominates, the prostate may form a tumour of enormous size, comparatively soft and elastic in consistence. Great lobes may grow out around the vesical orifice, and almost fill the lower part of the bladder. Masses the size of a cocoanut have been described. If, on the other hand, the glandular tissue is small in quantity, and is early replaced by fibrous stroma, there may be but little increase in size, or change in shape. But the substance of the gland may become as hard and as difficult to cut as scirrhus, and the resistance offered to the passage of the urine may be as serious as when the gland is of enormous size.

In enlargement of the prostate, pure and simple, the changes that are produced by the fibro-adenomatous growth are the only ones to be described. But when the enlargement is complicated by persistent urinary troubles, there are others, too, which are perhaps even more important from a clinical point of view. They arise from the venous congestion and the septic inflammation of the bladder and urethra, which are always present in these cases. Some of these additional changes, such as thickening of the mucous and sub-mucous layers of the urethra, solid œdema, thrombosis, and extravasation of blood, leave sufficiently clear traces behind them. They are as evident post-mortem as they were during life. Others disappear as soon as the circulation fails. The prostate is a much more vascular organ than is usually believed; and even when the inflammation is confined to the mucous membrane covering it, it is always more or less engorged. The hyperæmia and exudation spread into it from the affected surface: it becomes swollen, and the urethra, running through it, is compressed, and may be completely closed. Acute retention of urine, in cases of enlargement of the prostate, is nearly always due, in the last instance, to congestion.

After death, when the blood pressure has fallen, the swelling due to congestion disappears to a very large extent, and the gland then bears a very small resemblance, in point of size, to what it was during life. Some idea of the difference 
FIG. II.

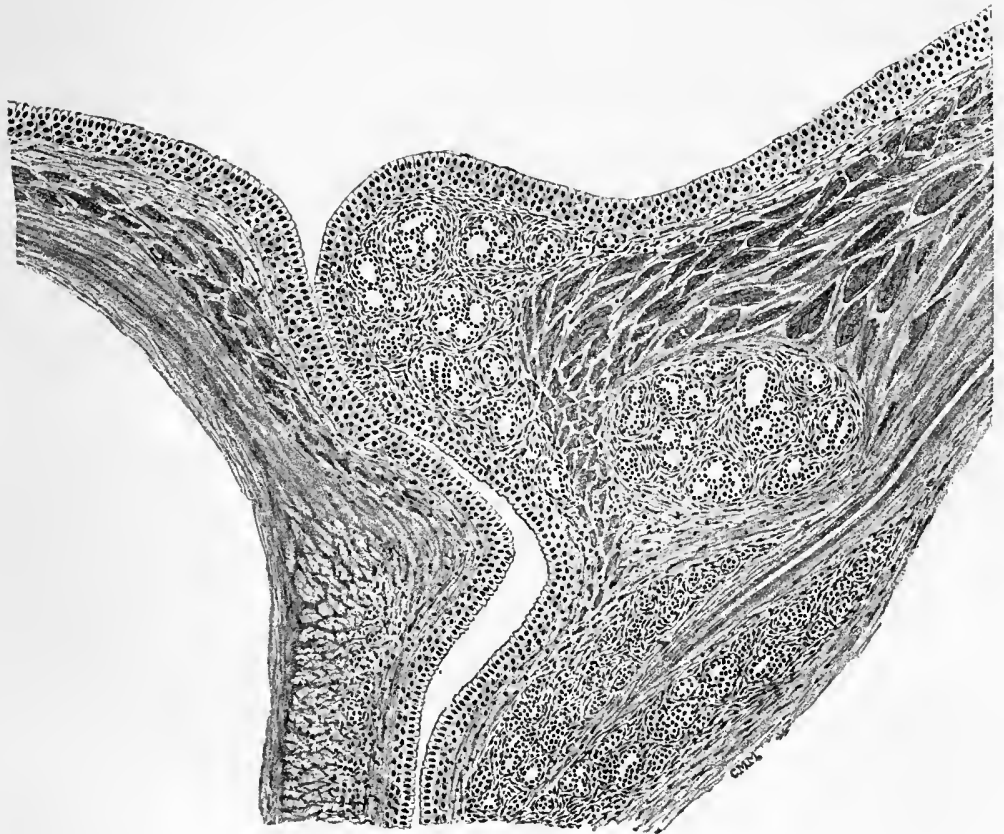

Median sagittal section through the neck of the bladder of a man forty-nine years of age, showing the growth of the adenoma up the posterior wall of the urethra into the neck of the bladder. Seen from the inside the vesical orifice was markedly crescentic in shape, as if the median lobe were projecting forwards into it. 

may be formed from the way in which portions of the prostate, removed during life, shrink and diminish in size in the course of a few hours; and also from the improvement in the symptoms, especially in the power of micturition, which so often follows partial operations upon the prostate, such, for example, as perineal puncture. It is not uncommon to find that a urethra which was so compressed, that it was impossible to pass a soft catheter down it, after a few days, when all the congestion has subsided, will admit one with ease.

The rate of growth may be uniform. More often it is very irregular, one part increasing more rapidly than those around it, and pressing upon them and even displacing them. A group of tubules, for example, with the stroma that invests them, lying in one of the lateral lobes, or in the median, begins to grow at a disproportionate rate. For a short time the glandular tissue is in excess: the cells multiply more rapidly, and the alveolar spaces enlarge more quickly. Then the investing stroma begins to increase in quantity. Soon the gland tubules become compressed and flattened; the cells undergo fatty degeneration, and disappear; the fibrous tissue becomes thicker and denser, and at length it grows into a hard rounded mass, with a laminated structure, separated by the way in which it has developed from the tissues around it, and exerting such pressure upon them that, if exposed on the surface of a section through the gland, it starts out from its bed of itself.

These are the so-called "prostatic tumours," which are erroneously held to be analogous with the fibroid tumours of the uterus. They occur in greater or smaller numbers in nearly every case of enlargement of the prostate. "These tumours do not appear to affect any particular part of the prostate more than another, and may be found in any part of it. Perhaps they are more numerous in the lateral lobes, especially at their posterior extremities, than elsewhere. Occasionally they are embedded in an enlarged median portion. It often happens that the small multiple eminences so frequently seen at the neck of the bladder, in the site of the uvula, are due to these small tumours, there situated under the mucous membrane and a few sub-mucous fibres, there being no enlargement of the median portion. . . . . Rarely 
they are found in the anterior commissure of the prostate. Sometimes they appear just under the capsule, and so spring from the surface, carrying in an outward direction the capsule as a covering, but nevertheless escaping altogether the contour of the gland, and looking almost like an independent or outlying formation. Occasionally they are really outlying, i.e., separated by an interval from the prostate itself. A space of half-an-inch has been seen to intervene between such a tumour and the adjacent gland, a narrow line of ducts with other vessels and a little tissue alone connecting them " (Sir H. Thompson, "Diseases of the Prostate").

The size of these masses is very variable. They may be no larger than a hemp seed, or they may be the size of a hazel nut. They may be single, and quite isolated, or they may be grouped together into compound masses, which may be so large as apparently to make up the whole prostate. Around the whole is"the thick, laminated, adventitious capsule, formed in part from the hypertrophied cortex, in part from the stroma and flattened acini, the remnants of the unaltered portion of the gland, pushed to one side. In many instances these adenomatous masses are entirely separated from the structures around, but sometimes they are still connected over a wider or smaller surface, and they always appear to be under pressure, so that when a section is made through the gland, their cut surface projects above the general level. Socin (Billroth and Pitha, iii., II., 39) described them as adenomyomata, but they contain very little muscular tissue. There are many long fibre cells which somewhat resemble unstriped muscle, but they are not arranged like muscle fibres, and in all probability are of connective tissue origin.

These masses are not tumours at all, in the proper sense of the term. They are merely pronounced local growths even when they form apparently the whole of the enlarged prostate. True myomata do occur in the prostate at times, as Griffiths has shown, but they are entirely different.

The peculiarly isolated character which enables them to be shelled out so easily by pressure is simply the result of their mode of growth. I have on several occasions met with similar rounded isolated masses, denser than the surrounding parts, and as easily separated from them, in tumours, such 
FIG. III,

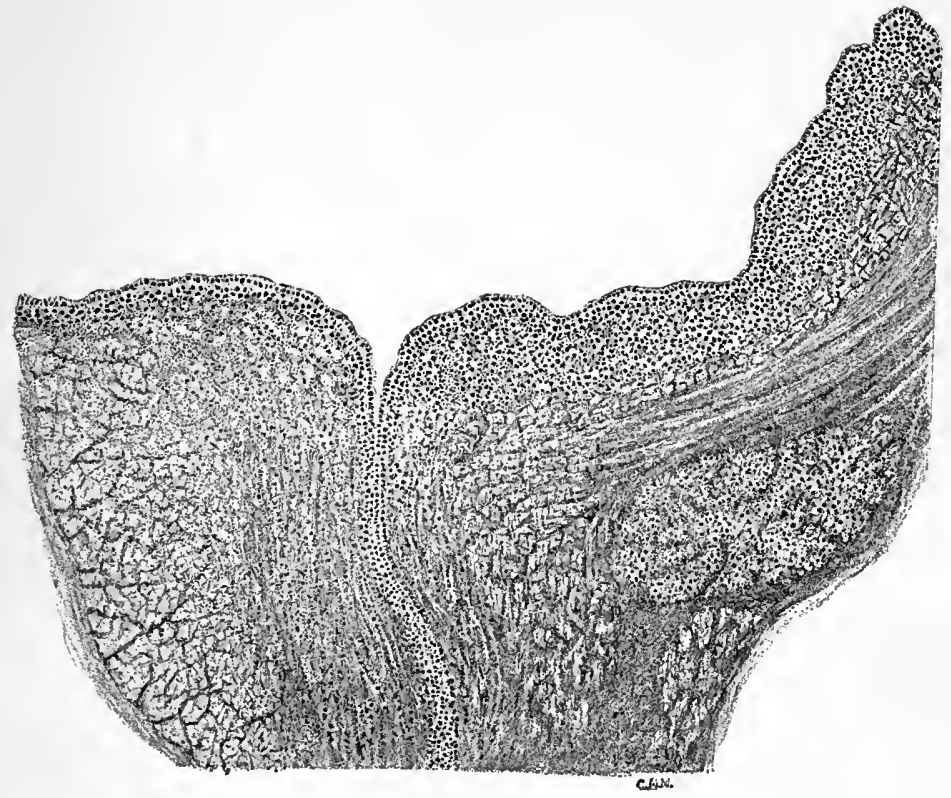

Sagittal section through the neck of the bladder, from a patient aged sixty-nine. The growth has extended up both the anterior and posterior walls of the urethra, and has reached quite to the back of the trigone. The muscular fibres around the neck are somewhat hypertrophied, and there is an indication of a commencing post-trigonal pouch. 

FIG. IV.

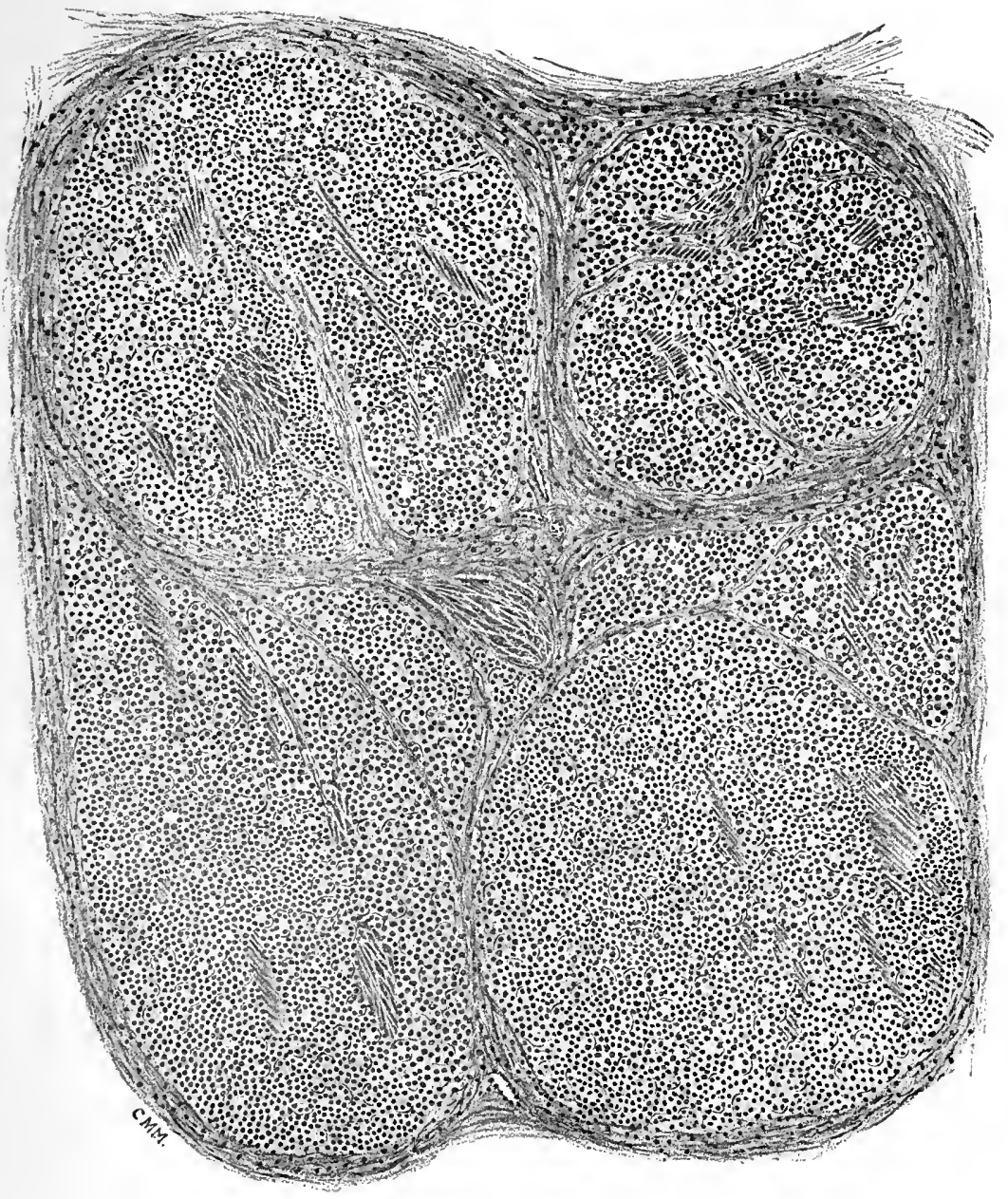

Microscopic section through the new growth, taken from the posterior boundary of the trigone of figure 3. It shows the peculiar character of the adenomatous tissue, composed apparently of irregularly arranged tubules, each with a basement membrane, a single layer of low columnar cells, and a few long fibre cells on the outer side, and here and there bundles of muscular fibre surrounded and isolated by the new growth. 

as fibro-sacromata, which possess somewhat of the same physical characters, and grow at unequal rates.

The growth of fibrous tissue in the enlarged prostate is often associated with marked alterations in the walls of the vessels, chiefly fibrous thickening of the intima and media. This association has been noted in other organs, in the kidneys for example, and the vascular change is considered by Ziegler and others to be the primary one (Griffiths, loc. cit.). It is to be noted, however, that the fibroid transformation which follows or accompanies the adenomatous growth in enlargement of the prostate is not merely fibroid degeneration, such as occurs in the kidney and other organs, as a consequence of or in association with general arteriosclerosis. There is a very distinct augmentation in volume, increasing considerably the pressure upon the parts around. The stroma does not grow so quickly as the tubules, and therefore is less in evidence at first, but there is actual growth, not mere degeneration. When the adenomatous tissue disappears there is no shrinkage in size: the increase is continuous, if slower, and is due to the continued growth of the stroma.

The adenomatous tissue is best developed and most conspicuous in that part of the enlargement which adjoins the bladder. In other parts the pressure caused by growth inside a tough unyielding fibrous sheath leads to early obliteration of the tubules. The immense power of resistance possessed by the combined capsule and sheath in the neighbourhood of the triangular ligament is probably the cause that determines the upward direction always followed by the enlargement.

Physical characters. - The prostate never grows downwards towards the triangular ligament, or invades that part of the urethra which is developed from the genital eminence; nor does it spread through its sheath, though isolated growths may carry this to an immense distance in front of them. When, therefore, it enlarges it raises the neck of the bladder from the triangular ligament, and alters the relations that it bears to the floor of the pelvis.

Size.-Sir H. Thompson considers that a weight of seven drachms fairly indicates that there is some enlargement when 
found in a subject over sixty years of age, though he has seen a prostate weighing less than six drachms which afforded unmistakable evidence of increase. Clinically, the size can only be estimated by measurement of the urethra and of the prostate itself through the rectum. The length of the prostatic urethra is about $3 \mathrm{~cm}$.; if it measures 3.5 there is enlargement.

Both methods should always be employed. Occasionally in enlargement of the prostate the first portion of the urethra is dilated, and if a catheter is passed, urine begins to flow long before the bladder is reached. Rectal examination in such a case corrects the mistake at once.

Shape.-The external shape of the prostate is altered when it enlarges. At first it becomes more uniform and rounded; the tension inside the sheath increases, and the cross section at the base becomes circular. Later it may become irregular.

The length is nearly always increased, sometimes so much that the finger introduced into the rectum cannot reach above it, and the first portion of the urethra measures seven or eight $\mathrm{cm}$.

The transverse and antero-posterior diameters are also increased, sometimes one, sometimes both, according to the direction of the enlargement. The notch between the upper ends of the lateral lobes behind is nearly always obliterated, and the groove that lies between the prostate and the bladder partly filled up. In extreme cases outgrowths of various shape and size make their appearance around the base of the gland, giving it an irregularly lobulated shape; but these are not nearly so common as the outgrowths that project into the urethra and bladder. Exceptionally they are pedunculated.

Varieties of enlargement.- The shape of the interior and the relation that the gland bears to the neck of the bladder and the first portion of the urethra are more variable.

I. The lateral lobes only may be enlarged. Usually they form two great ovoid masses, one on each side of the urethra, with their inner surfaces flattened, or even slightly convex, where they face each other. The prostatic urethra is reduced to a cleft between them, measuring from fifteen to twenty $\mathrm{mm}$. antero-posteriorly. At the posterior end it may be a little wider, owing to the projection into it of the veru 
montanum. The distance of the vesical orifice from the triangular ligament may be merely increased without its shape or plane being altered, the growth being mainly if not entirely extra-vesical, or the upper ends of the lobes may project upwards, one on each side of the opening through the muscular coat, into the cavity of the bladder. When this occurs they usually lift up between them a fold of the mucous membrane, and raise the orifice above the level of the trigone. When one lateral lobe, as is not uncommon, is enlarged to a greater extent than the other, the urethra is deflected towards the side of the smaller; and when their surfaces are nodulated from the projection of so-called prostatic tumours it may become very tortuous and irregular.

2. The enlargement may begin in the anatomical median portion. If the growth is limited to this the shape of the vesical orifice and the distance between it and the triangular ligament may not be altered, although the latter is usually slightly increased. The enilargement lies behind the urethra, outside and beneath the circular muscular coat of the bladder, and may be purely extra-vesical. As it increases it spreads backwards, below the trigone, filling up the notch between the two lateral lobes, and giving the posterior surface the characteristic rounded outline of the enlarged gland as felt through the rectum. Usually it presses forwards into the urethra as well, carrying the veru montanum in front of it, so that the cross-section of the canal becomes tri-radiate.

3. The enlargement may begin at the orifice of the bladder, in the posterior median line. At first sight this may be taken for a protrusion from the median portion; it is, however, entirely distinct. The cause of the enlargement is an upgrowth of adenomatous tissue in the sub-mucous coat of the urethra at its junction with the bladder. Between this and the median portion there is the layer of circular muscular fibre prolonged down on to the urethra from the bladder. The median portion may or may not be enlarged at the same time. Sometimes it can be seen in a sagittal section, lying by itself. In the later stages it is lost in the mass that is developed in the posterior wall of the urethra.

The size and shape of this growth have a very important bearing upon the outlet from the bladder. Even when quite 
small it raises this, and makes it crescent-shaped instead of circular. As it gets larger the posterior lip becomes thicker and more prominent. Then it grows out into a rounded sessile mass behind the opening; and at length, as it projects more and more, it forms a pedunculated lobe, which is often regarded as a product of the enlarged median portion.

In other cases the adenomatous tissue not only grows up behind the orifice, but extends around it as well. A thick semi-circular or horse-shoe shaped rampart may be formed in this way; and this again may grow out into lobes, especially if it is associated (as it usually is) with upgrowths from the enlarged lateral lobes below. Sometimes there are two of these upgrowths, one on either side; sometimes there is a third behind as well; and they may attain a very considerable size, and meet together over the orifice, so as almost to cover it when the bladder contracts.

Occasionally the elevation around the orifice forms a complete ring, the adenomatous growth having spread to the front from the sides, or extended up the anterior wall of the urethra from below. Exceptionally the anterior lip is thickened by itself, or grows out into a sessile or even a pedunculated mass. In extreme cases not only the margins of the orifice, but the surface of the trigone and the contiguous parts of the interior, are covered with growths of various shapes and sizes, usually more or less rounded, sessile, or with pedicles of different degrees of thickness, according to the extent to which they project. Masses the size of a Tangerine orange may be seen in every museum, and specimens as large as a cocoa-nut have been described. Whenever they occur they always lie on the inner surface of the muscular coat, covered over only by mucous membrane.

Whether an anatomical median portion exists or not is, so far as these outgrowths are concerned, entirely beside the question. In the specimen from which fig. 2 was taken there was a median projection at the junction of the bladder with the urethra, and the orifice was crescent-shaped instead of circular. But this was due to an entirely independent growth of adenomatous tissue in the mucous and submucous layers. The median portion, which happened also to be slightly enlarged, was quite separate and lay some little distance apart. 
A more advanced stage of the disease, from a patient 69 years of age, is shown in fig. 3. In this the growth spread not only up the urethra, but into the bladder. The whole trigone was invaded by it, up to the posterior boundary; and the level was raised in such a way as of necessity to leave a post-trigonal pouch. The microscopic structure, as shown by a section taken from near the base of the trigone (fig. 4) is almost the same as in the former case. In places, however, the glandular tissue has invaded the muscular layer. Here and there, surrounded by newly-formed masses of adenomatous tissue, are a few scattered bundles of muscular fibre. The circular muscular coat of the bladder is considerably thickened (it has had unusual work) and forms a welldefined layer which is continued down into the urethra. The growth in question lies upon its inner surface.

Infuence of enlargement of the prostate upon the urethra.-The shape and direction of the prostatic portion of the urethra are differently affected in these cases. The length is always increased and may even reach seven or eight $\mathrm{cm}$.

So far as the lower half is concerned the lateral lobes are the dominant force. If they are enlarged the urethra is compressed from side to side, and slightly elongated. The cavity may be reduced to a narrow slit, and the walls so closely pressed together (especially when there is any congestion) as to present a serious obstacle to the passage of the urine. In the upper part, nearer the bladder, their increase has not so much influence.

Enlargement of the median portion by itself leads to widening of the transverse diameter with protrusion of the veru montanum. If the lateral lobes are enlarged at the same time, they grow forwards, away from the median portion, and do not prevent the widening of the posterior part of the urethra. In this case they merely regulate the length of the anterior of the three rays into which the cavity of the urethra is compressed.

When there is an upgrowth of adenomatous tissue in the posterior wall of the urethra, not only is the length increased, but the direction and shape are materially altered. The orifice of the bladder is carried forwards, the urethra no longer follows an uniform curve, and a sharp angle is deve- 
loped under the projection. An ordinary metal catheter gliding along the posterior wall is stopped, and requires either to have the handle depressed or the curve increased before the point can ride over it.

When this upgrowth is accompanied by uniform enlargement of the median and lateral lobes it sometimes leads to permanent dilatation of the prostatic urethra. As it grows it spreads forwards and outwards as well as upwards into the bladder, and holds the sides of the urethra apart. If they are soft and flaccid the canal collapses, and merely its shape and direction are changed. If, on the other hand, they are dense and rigid, and especially if the median portion below is enlarged, so that the sides below the growth are held apart as well, the canal is kept open and a permanent cavity formed. This space is often capable of retaining several drachms of urine, and sometimes is of such a size that a short-beaked sound can be rotated in it without diffculty. I have known it, owing in part to its distance from the meatus, mistaken for an empty and contracted bladder.

As the true sphincter of the bladder is situated lower down, at the apex of the prostate, incontinence of urine does not occur in these cases, as might have been expected.

In one patient, however, under my care, in whom this condition was present, and who ultimately died from slowly progressive softening of the brain, true incontinence did make its appearance towards the end of life. The urine dribbled away, so that the bladder, which had been able to retain as much as fifty ounces, never held more than five or six. In this instance the sphincter ani was paralyzed as well.

Possibly this may have been the explanation in Mercier's four cases, in which the bladder was found empty and contracted post-mortem, while the prostate was enlarged and the internal meatus patent. In any case, however, as Sir $H$. Thompson has pointed out, true incontinence such as this is a very rare event.

The frequency with which enlargement occurs.-According to Sir H. Thompson enlargement of the prostate is met with in about one-third of the number of those who pass sixty years of age. The proportion, however, of those who suffer 
from it is very much smaller, probably not more than one in ten.

Frequency with which the different portions are affected.-It is very difficult to form an idea of the relative frequency with which the different portions are affected. Museum specimens are of little help. They are nearly always collected with a definite object, because the disease they illustrate is rare, or there is some special interest attached to it, or the specimen presents some unusual feature. And nearly always they illustrate only that stage of the disease which is no longer compatible with life, very rarely the beginning or even that period in which there is hope of operation or palliative treatment.

Vignard ("De la Prostatomie et de la Prostatectomie," Paris, I89o), analyzing the specimens in Civiale's Museum, with the view of ascertaining the frequency with which an isolated valve-like growth occurs, classifies them as follows:-

General hypertrophy, without distinct obstruction at the neck $\quad \ldots \quad \ldots \quad \ldots \quad \ldots \quad$ ro

$\begin{array}{llll}\text { With a distinct valvular growth } \quad \ldots & \ldots & 9\end{array}$

$\begin{array}{lllll}\text { Hypertrophy of the lateral lobes only } & \ldots & \ldots & 6\end{array}$

Partial hypertrophy, limited to the neck $\quad \ldots \quad 3$

28

General hypertrophy, therefore, being present in (approximately) 68 per cent.; a valvular median outgrowth by itself in only II per cent.; and in conjunction with general hypertrophy in 32 per cent. As Vignard points out, these statistics require to be taken with a certain amount of caution, as it is probable that specimens exhibiting hypertrophy of the median lobe would have been preserved to the exclusion of others from the interest attached to them.

Watson, on the other hand ("Operative Treatment of the Hypertrophied Prostate"), states that out of thirty cases that he figures the median enlargement formed the chief obstacle to urination in twenty-seven, and in ten out of thirty absolutely the only one. 


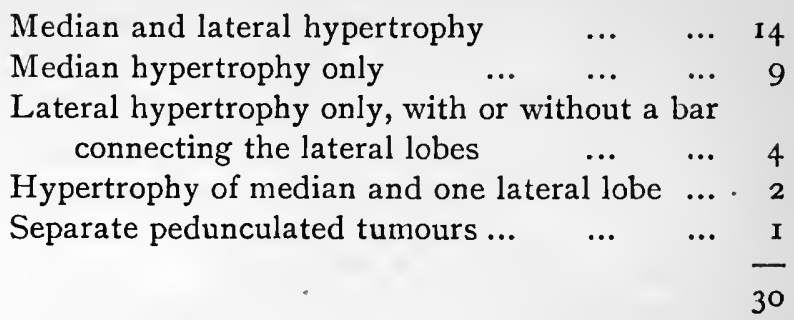

Vignard, therefore, in a series of favourably selected museum specimens (in all of which there had been retention) finds that in only 43 per cent. is there a median (or lateral) tumour or valve at the neck causing obstruction. Watson describes the median enlargement as forming the chief obstacle to urination in no less than 90 per cent.

Watson's results agree much more closely than Vignard's with those published by Sir $\mathrm{H}$. Thompson, drawn from four of the principal museums in London, and with those of Dittel. It is interesting that they all agree in regarding lateral enlargement by itself as forming a very small proportion of the whole.

It is probable that urinary symptoms were present during life in the majority of these cases, if not in all.

Statistics drawn from cases of enlargement of the prostate taken indiscriminately without reference to urinary difficulties agree with these fairly well. I have examined over a hundred taken in this way, and find that in at least four.fifths all parts of the gland are affected to a greater or less extent. The lateral lobes often show it least, because of the amount of fibrous stroma they contain, but their minute anatomy leaves no doubt as to the alterations they have undergone. It is the exception to find definite enlargement of one lobe without the rest being involved to some extent.

Evidence of this kind, however, is of little value in the question of treatment. The majority of the cases of enlargement of the prostate present no symptoms. If any importance is to be attached to the relative frequency with which the various lobes are affected, only those cases should be considered in which difficulty of micturition is present.

In the Hunterian Lectures upon "The Operative Treatment of the Enlargement of the Prostate" (I892), I published 
full details of all the cases of supra-pubic prostatectomy that had been performed up to that date, not only those that had appeared in the medical journals, but many that were privately communicated to me by surgeons in England and America. In eighty-six of these the part of the gland that caused the obstruction and was removed is specified. In forty-three per cent. there was either enlargement of the whole gland, or a "collar" around the orifice; in another forty-three per cent. the median lobe only is mentioned; and in the remaining fourteen per cent. it is stated that the lateral lobes were in fault.*

The proportion of those in which median enlargement alone is mentioned is considerably higher, not only than it is in Vignard's, but in the other statistics also. It must, however, be remembered that they were cases in which an operation was performed to remove an obstruction, and, therefore, the investigation was not carried farther than was absolutely necessary, and that in many instances, even when the lateral lobes were known to be enlarged, the surgeon may not have mentioned it, considering that as they did not form an obstacle to micturition, and were not interfered with, their overgrowth had no bearing upon the case. The proportion in which median enlargement with as well as without that of the lateral lobes ( 86 per cent.) existed is strikingly like that in the series collected by Sir H. Thompson, Watson and Dittel.

The main point is that in forty-three per cent. of cases that required operation the median upgrowth was found to be the sole obstructing cause, and in as many more a considerable factor in it.

* In these statistics, my own as well as those of other surgeons, I have, for the sake of uniformity, employed the term median lobe in its ordinary sense, viz., an enlargement under the mucous surface in the median posterior line. Probably in a large proportion, especially of those in which the overgrowth is described as taking the form of a collar, it was really an adenomatous outgrowth in the submucous layer, as described already, and had little or no connection with the anatomical median portion. 


\section{CHAPTER III.}

The Causes of Prostatic Enlargement.

The Time of Life at which the Enlargement Begins-The Analogy between Fibroid Tumours of the Uterus and Enlargement of the Prostate-The Connection between General Atheroma and Enlargement of the Prostate-The Theory of Compensative Hypertrophy-A Possible Solution in the Relation between the Prostate and the Testes.

Of all the theories that have been brought forward to explain enlargement of the prostate, and they are very many, not one is satisfactory. Most of them have been disproved already, times without number, and these I shall not mention. There are three, however, supported by the weight of such authority that they cannot be.lightly dismissed.

One is based upon a supposed analogy between prostatic enlargement and fibroid disease of the uterus. This was first suggested by Velpeau, and is supported by $\mathrm{Sir} \mathrm{H}$. Thompson, and Professor White, of Philadelphia.

According to another, enlargement of the prostate is merely one of the occurrences in a constitutional disorder which begins as arterial sclerosis and ends in fibroid degeneration, affecting the genito-urinary organs in an especial manner. This has the support of Guyon, and following him, the whole French school.

The third has been suggested by Mr. Reginald Harrison. According to this, the primary change is in the bladder; the enlargement is secondary to it, and developed as a compensatory measure.

The time of life at which the enlargement begins is a part of this question; for although old age is no longer regarded as a cause, there is a great tendency to speak of the enlargement as "senile," and to associate it with advancing years as one of the more or less likely events. In this matter it is especially necessary to distinguish the age at which the enlargement begins from that at which the symptoms it 
causes become so urgent that the patient is compelled to seek relief. Their may be a difference of many years.

It is generally stated, on the authority of Sir H. Thompson, that enlargement of the prostate never occurs at so early a period as fifty-three years of age. There is no doubt, however, that although it is unusual for it to cause symptoms that attract the patient's attention before then, the beginning may precede this by many years. McGill, for example (Brit. Med. Assoc., August, r 889), pointed out that the ages of two of the cases in which he operated were fifty-three and fifty-four respectively; and in the latter in which there could be no fallacy, the enlargement must have been present at or before the age of fifty.

I have met with several such cases, some of them younger still. In one (from which fig. 2 was taken) there was a distinct median projection into the neck of the bladder, due to glandular overgrowth at forty-nine years of age. Another, who was only forty-eight, was under the care of Dr. Henderson of Highgate. For some years he had been unable to empty his bladder without a catheter, and at the time that I saw him he was suffering from a typical attack of prostatic retention, caused by an enormous median lobe. A third, who was sent up to me by Dr. Walters, of Reigate, was only forty-one. In this case the mischief may have been exaggerated by attacks of chronic inflammation. However, on digital exploration through the perineum a distinct median lobe was felt, projecting into the urethra. For months before the patient had been unable to empty his bladder without a catheter; the median projection was divided freely, and six months later he had discarded his instrument, and could hold his urine for three or four hours without inconvenience. In a fourth, who consulted me first when he was fifty-three years of age, there was great dilatation of the prostatic urethra, showing that the enlargement was general, and therefore probably of some duration, and in addition an enlarged median lobe, which occasionally fell over the orifice.

Many other similar cases at even earlier ages have been recorded by other surgeons. McGill, subsequently to his two other operations in June, 1890 , removed and identified as such a certain amount of prostatic tissue that was acting as 
an obstacle to the outflow of urine from a patient only thirtyfive years of age (Mr. Moynihan, Leeds Gen. Infirmary). One of Teale's patients (Brit. Med. Assoc., August, 1889) was only fifty-four. Meinhardt Schmidt's, at the time of the first operation, was only fifty-two, and the symptoms had already lasted a very considerable time. Packard writes to me that in one of the cases mentioned in his paper on suprapubic cystotomy, read before the American Surgical Association, there was a very large prostate projecting into the bladder, although the man was only forty-three. White, of Philadelphia (Medical Neres, Oct. 13, 1890), found an enlarged and distinctly pedunculated median lobe in another patient of the same age. Belfield, of Chicago ( $A$ mer. Four. Med. Sciences, Nov., I89o), removed a mass the size of a walnut from the left lateral lobe of a patient forty-nine years of age, and he mentions several others-Dunn's, for example, in which a tumour was excised from the lateral lobe in a man of forty. five; Iversen's, in which the age was only thirty-six; and one by Mudd, of St. Louis, who exhibited at the Association of Urinary Surgeons in June, 189o, an enormously overgrown prostate, proved by microscopical examination not to be the result of malignant disease, taken from the body of a negro at the early age of twenty-seven.

When instances of this kind are met with in such numbers, at such various ages, it is clear that senility has little or nothing to do with enlargement as a cause.

If it were so the tendency to enlargement would increase, not perhaps in proportion, but still would increase as men grow older. This is not the case. As Sir H. Thompson has shown, enlargement rarely commences after seventy. Where it exists the disease has generally made considerable progress before seventy or seventy-five. Consequently it is met with but infrequently in later years, and is exceptional after eighty or eighty-five. Sir G. Humphrey states that of seventy-two persons between eighty and ninety, only seventeen, and of thirty above ninety, only one suffered from urinary troubles referable to enlargement of the prostate.

One deduction must be made from these figures. Enlargement of the prostate does not occur in all people; nor does it cause urinary symptoms in the majority of those in whom it 
does occur. Consequently it is possible that many who reached these advanced ages were among those who were exempt either from the disease itself, or the consequences to which it gives rise, and that those who suffered from it died at an earlier period because they suffered. Still, even when full allowance is made for this possible source of fallacy, it is a striking fact that extreme old age, instead of being more susceptible to this disease, is to a great extent exempt from it.

On the analogy between the growth or growths which make up enlargement of the prostate and the fibro-myomata of the uterus.-The view that these are analogous has received, and still receives, the highest support.

It was first suggested by Velpeau, on the ground that there was a striking resemblance between them in situation, structure and mode of growth. The prostatic growths are fibrous tumours in exactly the same sense as the growths in the wall of the uterus are fibrous tumours of the uterus. They are developed in the substance of the prostate, and sometimes acquire a volume much greater than the structure from which they spring, like the growths in the uterus. They become pedunculated, and project into the cavity of the bladder precisely in the same way that the polypi of the uterus grow out into the cavity of the uterus; or they may project, just as these do, towards the perineum, the side of the pelvis, or the rectum.

Sir $\mathrm{H}$. Thompson lends this opinion the weight of his authority not only for these reasons, but because the observations of modern anatomists confirm the view that the analogue of the uterus, or rather of the uterus and vagina combined, is in the male the prostatic vesicle or utricle. "The prostate, although not of itself the absolute equivalent of the uterus, contains in it the utricle, situated as this cavity is in the centre of the organ." The resemblance between the two organs in structure (they are both formed mainly of unstriped muscular fibre), the identical histological character of the growths (they differ only in the fact that the glandular part is more marked in the one than in the other), the close similarity in the relations that they bear to their respective organs (they may be encapsuled tumours, shelling out with 
ease, or polypoid outgrowths, or gigantic masses far exceeding in size the structure from which they spring), and the fact that the two organs are alike subject to considerable hypertrophic enlargement, with or without tumour formation, are all regarded by him as strong evidence in favour of close analogy. Moreover, the age at which these growths are most common is almost the same for the two sexes, if the difference in the duration of sexual life is taken into consideration; they are most frequent in the one as in the other during the latter half of the reproductive period; they seldom begin after this is past.

Prof. White, of Philadelphia (" Annals of Surgery," I 893), accepts this theory provisionally, and certainly there is more to be said in favour of it than there is in favour of the others. But there are arguments against it which are simply unanswerable.

It is not the case that it is supported by the facts of development. The homologue of the uterus is not the prostate, but the prostatic utricle, an entirely independent structure, which is included in the prostate gland merely by an accident of growth, and which has never been shown to take the least active share either in tumour formation or in general enlargement of the prostate gland. The homologue of the prostate, if it exists in the opposite sex, is to be found in the perineum, near the lower ends of the ducts of Gärtner.

The histology of the growths does not support it. Uterine growths originate as fibro-myomata. They have little or nothing to do with the mucous membrane or the glands that it contains. Enlargement of the prostate, on the other hand, is glandular from the first; it begins and spreads as an adenomatous growth, and even the rounded masses that it contains, with few exceptions, originate in the glandular tissue.

Both organs, it is true, contain unstriped muscular fibre (though the proportion in which this is present and its arrangement and uses are exceedingly different), and the growths which originate in them resemble each other to a certain extent, it is true, in shape (they are usually rounded), and in relations (sometimes they are encapsuled, sometimes they assume a polypoid form), but these peculiarities are by 
no means confined to these organs or their growths. Unstriped muscular fibre occurs in abundance elsewhere; rounded encapsuled masses that shell out when they are exposed by section, are by no means rare in fibro-sarcomata ; and tumours, when they project into the cavities of other viscera, not unfrequently become polypoid in shape.

That both these organs should be liable to tumour formation and enlargement during the latter half of the reproductive period, when the first flush of full sexual activity is over, is of great significance in many ways ; but it is scarcely suffcient to recommend the adoption of this theory, even provisionally.

Enlargement of the prostate may bear the same relation to the testes that fibroid disease of the uterus does to the ovaries. But the fact that they bear the same relation to two different organs is no proof that they bear any relation to each other.

On the theory that enlargement of the prostate is mevely one of the events that occur in general atheroma.-According to Guyon ("Leçons Cliniques sur les Affections Chirurgicales de la Vessie et de la Prostate"), and Launois ("De l'Appareil Urinaire des Viellards," Paris, I885), enlargement of the prostate is not a local affection, or the cause in more than a limited degree of the changes in the structure of the bladder. and the other urinary organs that accompany it. It is part of a general disorder of common occurrence in advancing age, characterised by the substitution of rigid fibrous tissue for active muscular and glandular structure; in other words, part of a general sclerosis affecting the genito-urinary organs in particular, and beginning in the vascular system, but not confined to it.

The prostate, so it is argued, becomes altered in structure. Hard, unyielding fibrous tissue is slowly but steadily substituted for the glandular part.

The bladder is affected in a similar manner. Hypertrophy: of its muscular coat and condensation of its mucous and submucous ones are normal events of old age. As the structure of the prostate is altered that of the bladder is altered too. There is over-production of fibrous tissue, which spreads everywhere in the intermuscular spaces, and in the mucous 
and sub-mucous coats. It increases more and more until at length great columns and ridges stand out from its surface.

The kidneys and ureters undergo a similar change. The cortex atrophies, and the capsule, or the vessels, or the glomeruli, or all together, become thickened and hardened by the development of rigid fibrous tissue.

Everywhere throughout the urinary tract there is the same widely spread and general sclerosis. It begins in the vascular system as endo- and peri-arteritis, and is aggravated by repeated attacks of chronic and sub-acute inflammation. Everywhere there is the same change pointing to diminished vitality.

Sclerosis precedes obstruction to the flow of urine and paves the way for the inflammatory changes that always follow. The arteries are the first to suffer, and the loss of force entailed by their rigidity leads to passive congestion and venous dilatation.

All other organs in the body suffer more or less in the same way. Enlargement of the prostate never occurs without general atheroma.

Enlargement of the prostate and failure of the muscular strength of the bladder are coincident events of the same disorder. The one is not the cause of the other. Sometimes one is the more prominent, sometimes the other; and all the symptoms that are ordinarily regarded as the consequence of enlargement ("la prostatisme") may occur without enlargement. It follows that it is useless if not dangerous in a case of vesical atony to rush into all kinds of therapeutic measures that appear capable of waking up the contractility of the muscular fibres. These either no longer exist, or else are so weakened and compromised by sclerosis that they can no longer respond.

The evidence against this view, which would prohibit any but the simplest palliative treatment, is to my mind insuperable. It is true, as Sir Benjamin Brodie pointed out, that enlargement of the prostate and atheroma frequently occur together; the time of life at which they are both most common is the same. But this does not show in the least degree that the one is the cause of the other.

Enlargement of the prostate does not begin as fibroid 
degeneration, but as glandular overgrowth. Arterial sclerosis and passive congestion cannot cause this; and still less can they cause the growth of the masses that sometimes almost fill the lower part of the bladder.

Enlargement of the prostate is compatible with perfect health, and may continue even to extreme old age without its existence being known. Those who suffer from urinary troubles form but a small proportion of those in whom enlargement occurs. The vast majority are free, and though some may be atheromatous, many live on with no evidence of vascular degeneration other than that which is the normal accompaniment of healthy old age.

Enlargement of the prostate may be present and may have already attained considerable dimensions long before the age at which atheroma occurs; and, on the other hand, after a certain time of life, if it is not present already, it rarely begins.

Nor is it the case that enlargement of the prostate and loss of power of the bladder of necessity occur together. The bladder in a large proportion of cases retains its power in the face of even considerable enlargement. And if the enlargement is removed, and it is not too late, the bladder may regain the power it has lost. In what proportion this occurs I shall discuss later. Among the earlier cases it was said to be only in one-third of those in which supra-pubic prostatectomy had been performed, a proportion that is not surprising if the previous condition of the patient is considered for a moment. In later cases, and still more among those which have been treated by orchidectomy, the proportion is a great deal higher. But that is not the question here. If recovery occurs at all it is sufficient. If the obstructing growth and the loss of power of the bladder were both due to general fibroid degeneration and impaired vitality, removal of the one would have no effect upon the other.

On the theory that the primary change is in the bladder, and that the enlargement of the prostate is developed in compensation.-Mr. Reginald Harrison ("Lectures on the Surgical Disorders of the Urinary Organs") regards the prostate as chiefly muscular, and sums up its structure as consisting of unstriped muscular fibre, in which a considerable amount of tissue engaged in 
secretion is embedded. The enlargement he considers is developed to compensate for the sinking into the floor of the pelvis of the posterior wall of the bladder. This is the primary change and one of the consequences of advancing years.

"I have long felt that the solution of the question (the cause of the enlargement) would probably turn on a further recognition of the function of the part involved, and that thus the urinary apparatus might furnish another instance of a compensatory contrivance to meet exigencies arising out of structural deterioration.

"We have not yet sufficiently recognised the use of the prostate as a muscular support-primarily to the bladder and its contents, and secondarily to the adjacent parts - a support which in the event of muscular tissue failing must be supplemented by other means and other tissues.

"Assuming that from any cause, such as long retention of urine, habit, position of the body, or weakness connected with advancing years, the floor of the bladder sinks lower within the pelvis, relatively to the prostate, so as to offer some difficulty in expelling the last portion of the urine, the effect will be frequently repeated efforts in all the muscles immediately adjacent to a part of the bladder, which by reason of its connections and structure, has but little power of contracting. This will eventually lead, as I have endeavoured to show, to the development of a strong muscular buttress between the orifices of the ureters, and also, I believe, to similar changes in the muscular tissue so largely entering into the prostate and principally affecting its posterior segment.

"Quantity is substituted for quality, and as age advances structural deterioration is thus provided against."

The outgrowths which are so frequent and so large in connection with enlargement of the prostate, Mr. Harrison accounts for as the upheavings of a frequently contracting muscular ring.

This theory, like the other two, is untenable. The prostate, both as regards, its glandular substance and its stroma, is of sexual origin, and has to deal only with sexual functions. It develops with the testes, and atrophies if they are removed. 
It has nothing to do with micturition, which, if the prostate remains undeveloped or undergoes atrophy, takes place just as well as it does if the prostate is normal.

Nor is there any evidence that a sinking of the posterior part of the bladder is a prinary change; nor that when it does sink the prostate enlarges in compensation. This can only be imagined so long as the erect position is maintained and the prostate bears the same relation to the bladder that it does in man, whereas enlargement takes place in dogs and other quadrupeds, in which the anatomical relations are entirely different.

The present state of our knowledge does not enable us to say why enlargement of the prostate is prone to occur at a particular period of life, any more than it enables us to say why fibro-adenomata so frequently occur in the breast shortly after its evolution at puberty, or why fibro-myomata so often make their appearance in the uterus towards the latter end of its active sexual existence.

It is certain, from the facts of human as well as of comparative anatomy, that the normal development of the prostate is dependent upon that of the testes and vasa deferentia. If the testes are removed in early life, the prostate does not grow. If the vas deferens is not developed upon one side of the body, the prostate upon that side often fails too, even though the corresponding testis is present. If the testes are removed after puberty, the prostate shrinks and atrophies. There are exceptions, it is true, but they are not sufficient to prevent the acceptance of the general principle.' It is almost as certain that the enlargement depends upon some influence exerted by the same structures.

The evidence is fairly conclusive.

It has long been a matter of popular tradition that enlargement of the prostate has a great tendency to show itself soon after a second marriage contracted late in life. By itself this means very'little; the fact, if true, is capable of being interpreted in other ways. In conjunction with other evidence it becomes of high significance.

Enlargement of the prostate, as Sir H. Thompson and Sir G. Humphry have shown, is more uncommon in very old 
men, in whom it may be presumed the testes are no longer functionally active, than it is in younger ones, those for example who have just passed middle life. This may mean either that the prostate never became enlarged in the former, or that the overgrowth, which had developed without attracting attention, disappears again when the testes lose their power. Whichever explanation is adopted, this at least stands out, that the relative frequency with which enlargement occurs, diminishes as the activity of the testes fails.

But there is better evidence than this. It may be taken as proved that, as a general rule, simple enlargement of the prostate disappears within a few months after the testes have been removed. It is true that cases have been recorded in which no perceptible change has been found even after a year, just as there are a few instances known in which atrophy of the prostate has not followed castration in younger subjects. But neither in the one case nor in the other are the exceptions sufficiently numerous to invalidate the general rule.

It is almost certain therefore that this abnormal overgrowth must be in some way dependent upon the masterorgans of the male sexual system. In what way it is not possible to say. We know too little of the influence that one organ has upon the rest. Affections of the thyroid can produce the most profound structural alterations throughout the body. It is believed on experimental evidence that the pancreas has duties in connection with general nutrition entirely independent of its secreting action. The same is probably true of the kidneys. There is reason to believe that the condition of the ovaries has some influence in connection with mollities ossium; there are cases, at any rate, in which removal of the ovaries is stated to have been followed by recovery from this otherwise almost universally progressive disease. Removal of the uterine appendages, inducing the menopause prematurely, undoubtedly has an effect upon the growth of fibro-myomata of the uterus. And it may be that certain conditions of the testes usually occurring at that time of life (but sometimes earlier) may stimulate the prostate to increased and irregular growth.

In connection with this it has been pointed out by Griffiths 
in the Hunterian Lectures for 1895 (Lancet, March 30, I895), that the function of the testes is twofold :-

"It is of much interest to find that the undescended testes, though incapable of producing their special secretion of spermatozoa, yet are capable of exerting their peculiar and important influence upon the growth and development of the body. When only one testis fails to 'descend' to the termination of its destined course, the opposite organ having duly reached the scrotum, the person grows, as we often have the opportunity of observing, just as if both organs were in their natural position. The fully descended testicle produces spermatozoa in abundance and usually acquires a larger size than natural, due to what is called 'compensatory hypertrophy.' Thus, a person who has one testis natural and in the scrotum, whether the opposite be undescended or absent, is in possession of full virility. But when both testes fail in their descent they, as we have just seen, are incapable of producing spermatozoa, and in consequence the person is sterile. In spite, however, of the imperfection of the organs, such a person acquires all the external bodily characteristics of the male, and is in all respects, except in the power of procreating, like an ordinary man. His shoulders are broad, his pelvis is narrow, his beard and moustache and hair on the pubes are well grown, his voice is deep and manly, and his penis is large and well developed, though the scrotum is small and empty; so that the external appearance, except that of the scrotum, would give no clue to the condition. I know of one such person who is well formed and who is capable of erection and emission, but without issue. It is evident, therefore, that the influence of the testis upon the growth and maintenance of the characteristics of the male is a property independent of their power of producing spermatozoa; and the remarkable fact is established that the spermatozoa-producing work of the testes, possibly even their potentiality for that purpose, is not necessary for the establishment of the influence which those organs exert upon the rest of the body. It is further of interest that, although the natural involution of the testis in the aged is unaccompanied by any corresponding change in the system, yet if the testes in' an elderly person are removed the accessory sexual glands, 
at any rate, are found to undergo changes somewhat similar to those which are observed in eunuchs and in eunochoid persons.

"The function of the testes is, therefore, clearly twofold, viz., (I) to control and determine the development of the characteristics of the male sex, and (2) to produce spermatozoa for the reproduction of the species. These two functions are usually exercised together, but that the former may be exercised when the latter fails seems to indicate that the production of spermatozoa is the more specialised property and attained with more difficulty. In what manner is this sexual effect of the testes upon the body produced? Is it through the medium of the nervous system as an ordinary reflex, or is it through the medium of some substance produced by the seminal cells (whether they form spermatozoa or not) and absorbed into the system, which by influencing the nerve centres or in some other way controls growth and nutrition? Brown-Séquard tried upon himself, when he was seventy-two years of age, the effect of the subcutaneous injection of a watery extract of the testes of a vigorous dog two or three years of age, and relates that after five daily injections he lost his feebleness, felt many years younger, and was capable of doing more work. The testicle extract has since been used in various diseased conditions, chiefly those associated with nervous debility, but with only temporary results. During the last few years a watery extract of the thyroid gland has been administered with signal success in myxœdema, in which disease the thyroid gland atrophies and ultimately disappears. The disease myxœdema arises from the want of the influence of some unknown substance, which the thyroid gland, as is supposed, elaborates, upon the nutrition centres of the central nervous system. It may be that the testis in like manner elaborates, irrespectively of its spermatic secretion, some chemical substance which by a similar influence not only controls the growth and development of the body at puberty but maintains the manly characters then acquired throughout life."

So far as the prostate is concerned there is evidence to show that its growth and nutrition are independent of the spermatozoa-producing power of the testes. The accessory 
sexual glands may be well developed even when the testes fail to descend. And Lütkens (Deutsch. Med. Woch., 5, 1894) has shown that reduction in size of the enlarged prostate may follow removal of the testes even when no living spermatozoa can be found. It seems, therefore, that the nutrition of the prostate is controlled by some other property resident in the testes, distinct from this. But it does not follow that that property is the one mentioned by Dr. Griffiths, which determines the development of the secondary sexual character-, istics. It may be distinct from this one also.

It must be remembered that the relation that exists between the testes and the accessory sexual organs is not identical with that which exists between the testes and the secondary sexual characteristics. It is of a different character altogether. For while removal of the testes in infancy prevents the development of both, the same operation performed after puberty has no effect upon the latter. After castration performed in adult life, the secondary sexual characters, the general appearance, the voice and figure remain unchanged. Once developed they are permanent and independent. The vesiculæ seminales, on the other hand, in the same circumstances shrink and contract, and the prostate atrophies. Removal of the testes not only checks the development of these accessory structures, but, if they have attained their full growth, causes them to atrophy and waste away.

There are some other points that deserve mention in connection with this. The testis on each side, with its accessory organs (the epididymis, vas deferens, vesicula seminalis, and corresponding half of the prostate) forms one complete functional group. But pathologically and genetically the testis is distinct from the others. The accessory organs are developed from the Wolffian duct with which the testis has no connection. If the kidney and the vas deferens are wanting upon one side of the body, the vesicula seminalis and the corresponding half of the prostate are generally wanting too, but the testis may be fully developed. And the liability of the testis to be attacked by different diseases, such for instance as tubercle, is very different from that of the epididymis. The testis and its accessory organs work together and form 
one functional whole; but it seems probable, that so far as their growth and nutrition are concerned the several accessory organs (including in these the epididymis) are much more intimately connected with each other than they are with the testes. They may exist independently of the testis and the testis may exist independently of them. The fact that neither the normal growth nor the abnornal enlargement of the prostate appears to be necessarily associated with the spermatozoa-producing power of the testes helps to confirm this.

The question whether the influence which the testes possess over the normal and abnormal growth of the prostate is exerted through the medium of the nervous system, or by virtue of some unknown chemical product which they form and discharge into the circulation, is of great practical importance in connection with the success of unilateral orchidectomy. If the former view is correct, and it could be proved that each testis had sole control over the corresponding half of the prostate, or even that, without having sole control, it had a strongly predominating influence, removal of one testis or of one vas deferens might not unfrequently be sufficient. The corresponding half of the prostate, cut off from the organ that maintained it, would atrophy, and, in a certain proportion of cases, leave the exit from the bladder sufficiently free. On the other hand this would be most unlikely if the influence of the testes were due to some chemical substance produced by them and discharged into the general circulation.

This point, however, I intend to reserve until the results of bilateral orchidectomy and of other operations upon the structures contained in the spermatic cord have been considered. I would only remark that in determining a question of this kind, but little reliance can be placed upon the results obtained by experiments upon the lower animals. In many of these the prostate is from the first, and remains, bilateral. There are two separate organs, one on each side of the body. In man this is not so. There may be two separate organs at first, but in process of development they grow towards each other and fuse in the middle line; and the degree to which they fuse differs in different individuals. In some the lateral 
lobes remain quite separate from each other, so far as the glandular structure is concerned. In others, in whom the displacement of the gland tubules in the course of development has been more considerable, the two halves approach each other in the posterior median line and are more or less united. And in others again the fusion is carried so much farther that the bilateral character is lost and the gland appears to be a median growth.

I have recorded elsewhere (Lancet, Feb. I, I896) a case of enlarged prostate in a man who was a monorchid almost from birth. One testis had been destroyed in very early infancy. But this did not prevent the formation of a large median outgrowth that projected into the neck of the bladder and caused serious obstruction to the outflow of urine.

It is clear, therefore, that even if it were proved that the influence of the testes upon the prostate is exerted through the agency of the nervous system, and that the unilateral operation is followed by unilateral atrophy in animals, it cannot be relied upon to succeed with any degree of certainty in the case of man, although it may advantageously be tried when it is known beforehand that the enlargement involves the lateral lobes and does not affect the median portion. 


\section{CHAPTER IV.}

The Effects of Enlargement of the Prostate.

The Effect upon the Bladder-The Increase in the Amount of WorkCongestion of the Prostatic Plexus-Irritability of the Bladder-The Influence of Age, and of Cystitis-The Effect of the Enlargement upon the Kidneys.

THE gravity of enlargement of the prostate is due to the changes that it induces in the bladder and kidneys. That it is, directly or indirectly, the cause of these changes there is no doubt. They are met with constantly in association with enlargement of the prostate. They do not occur without it or without some similar cause, such as calculus, or neglected and inveterate stricture; and therefore, as might be expected they are rarely found in women.

The size and shape of the bladder are altered. It may be so small that it can hold only three or four ounces, or so large that it can contain as many pints. Its shape may be normal, or it may be conical, or pushed out into a great pouch behind, or studded with rounded sacculi. These sometimes consist of all the coats, but the largest and most numerous are formed of little more than the mucous membrane projecting under the serous layer from between the bundles of muscular fibre.

The inner surface may retain its natural colour, or it may be stained all shades from red to black by old and recent hæmorrhages. It may be smooth or marked by interlacing ridges caused by the thickened muscular coat beneath. The lozenge-shaped spaces between these may be mere shallow depressions, or they may lead, often by very narrow orifices, into sacculi filled with stagnant and often decomposing urine. The epithelial lining may be intact and preserve its normal smooth appearance, or it may be roughened, ulcerated, and coated over with an offensive and tenacious mixture of pus and phosphates. 
The walls may be rigid and thickened by the growth of fibrous tissue, so that they can neither relax nor contract; or they may be thin and flaccid. In the earliest stages there is sometimes a certain amount of true muscular hypertrophy, but afterwards power is always impaired. The muscular bands may undergo fatty degeneration and disappear, or they may grow out into great columns that project into the cavity of the bladder; but, as a rule, there is more fibrous tissue in these than muscle. Sometimes the mucous and submucous layers are stretched and thin. More often, especially when the bladder is small, they are thickened and indurated, or filled with varicose venous plexuses, which, in cases of retention, are plugged with thrombi in various stages of organization or decay.

The ureters and the pelvis of the kidneys suffer in a similar way. The mucous membrane is swollen and congested. In the later stages, when septic cystitis has set in, it becomes inflamed, and even ulcerated. The muscular coat is wasted, and to a great extent, replaced by fibrous tissue. Sometimes the walls are thickened; more often they are thinned and stretched until they sometimes attain an enormous size.

The kidney itself is smaller and harder than natural. The capsule is adherent and puckered on the surface. The cortex is atrophied and studded here and there with small cysts. The medulla is thinned, and the general texture is tougher and more resistant. Later, when septic organisms have found their way into the pelvis and spread through the lymphatics into the cortex, it may be riddled with abscesses. The primary change throughout is wasting of the active muscular and glandular structures. In their place, passive and inert fibrous tissue makes its appearance, and this either contracts, or yields and stretches, according to the degree and persistence of the pressure which is brought to bear upon it. Then, when the vitality of the tissues has become impaired and their power of resistance broken down, septic organisms enter and set up suppurative pyelonephritis.

The immediate cause of these changes is the upgrowth of the prostate at the neck of the bladder, partly by the mechanical difficulty that it offers to the passage of the urine, partly by the venous congestion that arises from the constant and 
prolonged straining. If there were no such difficulty or venous congestion, the entrance of septic organisms would lose much, if not the whole of its importance, for any organisms that did get in, would be expelled at once.

The effect of enlargement of the prostate upon the bladder.-It does not matter what part of the prostate is affected. It may be the median or the lateral lobes; it may be all three, or it may be the urethral portion only. Wherever it occurs it obstructs the exit of urine from the bladder and increases the work.

Every form of enlargement, for example, interferes with the action of the neck of the bladder. As micturition begins, the first part of the urethra relaxes, dilates, and becomes physiologically, as it is genetically, part of the bladder. So long as the gland retains its normal shape and size, this takes place without difficulty. When enlargement begins, no matter what may be the shape (though some are much worse than others in this respect), or when the prostate, instead of enlarging becomes hard and dense, expansion is checked; the sides of the urethra remain in contact, and the amount of resistance the bladder has to overcome is increased.

Every form again interferes with the level of the floor of the bladder. Enlargement of the prostate is always accompanied by an increase in the length of the prostatic urethra, especially of its posterior wall, and this necessarily raises the outlet. Growth cannot take place downwards because of the triangular ligament. It must take place upwards, and carry with it the neck of the bladder. So far as the anterior portion and the sides are concerned, this is not of material consequence; they too are raised, not so much perhaps, but sufficiently to prevent their becoming receptacles for stagnant urine. The posterior wall, however, stretching farther backwards, does not escape so easily. Its even gradual slope from above downwards is broken; the apex of the trigone is raised, and the part of the bladder that lies behind it becomes the lowest and the last to be emptied. So long as the muscular coat is equal to the task this does not matter; there is a little more work to be done in emptying the bladder, but if the tissues are healthy and not too old they grow stronger and do it. If, on the other hand the balance is upset, if the 
amount of resistance in front is increased too quickly, or if the strength begins to fail (and as Mr. Hurry Fenwick has shown the posterior wall of the bladder is thinner than the anterior), this part of the bladder, because it is the weakest and has most to do, gives way first, a certain amount of urine is left behind, and at length a pouch is formed.

The alteration in the shape and direction of the orifice has a similar effect to the alteration in its position. In the child the opening is circular, and when the bladder is full and micturition beginning, funnel-shaped with shelving margins. As the growth invades it, it becomes bent into the form of a crescent, the posterior lip enlarging and pressing forwards. into it, or elongated from before backwards, or irregular, according to the part and extent of the circumference involved. Later, when the adenomatous tissue spreads upwards and projects into the bladder, the alteration is much greater. A thick rim that is not by any means soft or yielding may be built up around it, or around the posterior half; a rounded mass may spring up from behind and hang over it ; or there may be two, one on each side with or without a fold of mucous membrane to unite them; or three, one on each side, and one in the middle line behind as well. Much more rarely there is a single one in front. Sometimes, as in fig. 3 , the growth spreads backwards upon the upper surface of the trigone; sometimes it grows out irregularly in all directions, forming lobulated masses of the most varied shape and size.

Every growth of this kind increases the amount of work thrown upon the bladder. They all raise the orifice, and so form a pouch behind, and they all increase the resistance in the prostatic urethra; but some are much worse than this. They may grow round and over the orifice, making it smaller and smaller until at length they close it altogether, in spite of the most strenuous efforts on the part of the muscular coat.

If, for example, there is a single lobe projecting upwards either from behind or one side, or if there are three arranged symmetrically around the orifice, micturition may be impossible unaided. As soon as the bladder begins to contract and the urine is pressed from all sides on to the exit, the lobe or lobes are shut down like a valve, and the more the muscles. contract the more tightly the orifice is closed. 
If the median growth, instead of projecting upwards into the bladder, juts forwards from behind into the urethra, below the orifice, the result is almost as bad. Only in this case the flow of urine is not stopped at the beginning, for at that time the muscles around the lower part of the bladder are relaxed, but later, when the bladder has partially emptied itself and these are beginning to take their turn. The immediate result is a dilatation of the post-prostatic part of the cavity, and this follows all the sooner because the apex of the trigone is in these cases always raised as well as prolonged forwards.

But this is not the only way in which enlargement of the prostate obstructs the exit of the urine from the bladder and makes its work more difficult.

Sooner or later all those in whom the prostate reaches any considerable size are certain to suffer from attacks of congestion, and, perhaps, thrombosis of the prostatic plexus of veins.

The arrangement of this plexus has already been described. In childhood and in youth it is only of moderate dimensions, and all the vesical and other veins that enter it are accurately valved (Fenwick). In old age it enlarges to an enormous extent; the valves disappear, and lying as it does almost at the lowest point of the venous system of the pelvis, surrounded by organs the blood supply of which presents the greatest variations, it is exceedingly liable to attacks of congestion and thrombosis.

Enlargement of the prostate, when it gives rise to any symptoms at all, always causes straining, and straining, frequently repeated, acting upon an intricate plexus of valveless veins, always causes congestion. Often the bowel is affected as much as the bladder. Hæmorrhoids and prolapse of the rectum are seldom wanting in cases of long standing prostatic enlargement.

So universal is congestion in connection with enlargement of the prostate and so grave are the consequences it entails that some have felt inclined to regard it as the cause not merely of the symptoms, but of the enlargement itself. This, plainly, it cannot be. No amount of congestion can lead to glandular overgrowth. 
The immediate effect of congestion is to narrow the exit from the bladder. The mucous membrane becomes thickened and swollen: the loose submucous tissue that enables it to glide over the subjacent muscle, becomes hard and dense; and the channel, already contracted by the enlargement of the gland, is narrowed still more. In many cases the orifice becomes completely closed. Congestion is the immediate cause of most of the cases of sudden retention of urine that occur in old men suffering from enlargement of the prostate. The resistance becomes too great, and the bladder cannot overcome it.

But this is not all. Besides obstructing the passage from the bladder and rendering the task of emptying it more difficult, congestion of the prostate increases in another way the amount of work that is thrown upon the bladder. It makes the bladder contract so much more frequently. The mucous membrane lining the neck becomes so sensitive that the bladder is never able to rest. No sooner do a few drops of urine collect than they must be expelled. The desire is so intense that it cannot be resisted, and so persistent that the muscular coat scarcely has time to relax.

This occurs to a greater or less extent (independently of cystitis which makes it infinitely worse) in nearly all cases of prostatic enlargement, but especially in those in which there are prostatic outgrowths projecting upwards into the bladder. These appear to act in the same way as true vesical tumours springing from the trigone. Owing to the hyperæmia that attends them, the mucous membrane becomes so sensitive that the least stimulus, even the presence of a few drops of urine, acts as a powerful irritant, and the bladder is kept incessantly contracting.

Now the effect that increased work has upon a muscular structure, such as the bladder, is not always the same.

So long as the patient is young and healthy, so long as he still retains the power of ready adaptation to changing conditions, increased work (always provided that the increase is not too sudden or too great) leads to increased strength. The muscles, called upon for greater exertion, respond by growing in size and strength; the walls of the bladder grow thicker; the muscular bands become more prominent and 
stand out as ridges, and the cavity diminishes a little in size.

But if on the other hand everything is not so favourable; if the amount of work to be done is increased too suddenly, or if the patient's constitution is beginning to give way, the result that follows is very different. The muscular coat of the bladder, called upon to do more than it is able to do, tries and fails. The fibres lose their power of contracting and waste. The cavity increases in size. The walls become thinned and stretched, the weakest part, and that on which the work falls heaviest, of course giving way the first; the power of expelling the urine grows feebler and feebler; more and more is left behind, until at last the bladder is only able to get rid of a few drops at a time and complete retention follows.

As a rule the presence of a mechanical obstruction at the exit is more likely to lead to dilatation of the bladder, increased irritability to diminution in size, but there are many exceptions. It is not uncommon, for example, as age advances and the conditions of life change, for the one to succeed the other; there is hypertrophy for a time and then dilatation and atony. And occasionally different parts of the bladder, owing to differences in strength, behave in different ways. That part of the wall, for instance, which lies between the ureters, and is exceptionally strong, may become hypertrophied, and give rise to what has been called the interureteral bar, while that which lies above and below yields and gives way.

Now when the prostate is enlarged the conditions are rarely so simple or uniform as this, even at the first. Other considerations, besides the mere amount of mechanical resistance which has to be overcome, enter in and introduce complications.

Old age is one of these. So long as health is maintained, compensatory hypertrophy is possible at almost any time of life. But as years go on it no longer takes place with the same degree of readiness and it never reaches the same degree of perfection.

The walls of the bladder in old people are naturally stiffer and more rigid; the mucous membrane is more dense and less flexible; the muscular coat is weaker; and the fibres, 
instead of responding to increased work by increased strength, waste and yield. A comparatively slight degree of resistance, particularly if it develops suddenly, leads to dilatation and atony; and even when the strength is increased at the first, it is only for a while.

Venous congestion is another condition for which allowance has to be made. The effect that it has in increasing the work of the bladder, by helping to block the outlet and by making the bladder so irritable that it is perpetually contracting, has been mentioned already. But, besides this, venous congestion, especially when it is long continued, has another and if possible more injurious influence still. It seriously weakens the muscular power of the bladder. Just at first, while the work to be done is still within its range, the muscular coat may become a little hypertrophied and columns and ridges may begin to stand out under the mucous surface. But inevitably as the congestion increases and the blood supply grows worse, the nutrition of the fibres fails; many of them undergo degeneration and disappear, and a condition approaching solid œdema sets in. The walls grow hard and rigid, the exudation that fills the interstices becomes organized, flexibility is lost, the bladder can neither contract nor expand as it should, and even though the walls grow thicker, the power of expulsion diminishes.

And above all, congestion increases the liability to inflammation. The interior of the bladder, especially the most sensitive and the most exposed portion, that which lies around the neck, is kept in a state of constant slight catarrh. The epithelial cells are badly developed and unable to protect the structures beneath, and the mucous membrane is swollen and œdematous, and filled with varicose and thrombosed veins. Now, so long as the bladder is healthy, its surface is impervious to organisms. I have known the pus from an appendix abscess flow through it for days together without affecting it in any way. Even if the surface is injured, scratched, for example, or rubbed, the liability is but little increased. But let congestion, or what is worse still, congestion and retention set in, the power of resisting infection is lost, and septic inflammation follows at once.

Enlargement of the prostate, therefore, whatever form it 
assumes, and without taking into consideration any of the complications that are nearly always present, invariably increases the amount of work by adding to the resistance in front, or by causing increased frequency of micturition, or by both together; and, at the same time, owing to the straining and congestion which it always brings with it, it impairs the nutrition of the bladder, weakens its power of expulsion, and renders it much more susceptible to inflammation. The result depends partly upon the rate and the amount of increase; partly upon the extent to which the nutrition is affected.

(a). In many instances the difficulty is so slight or is overcome so easily that there is never any serious amount of straining or congestion; the patient's attention is never attracted to it, and the condition of the bladder does not suffer. A sufficient degree of hypertrophy takes place, the walls become a little thicker and more rigid, the bladder loses a certain amount of its distensibility, that is to say, it cannot hold without inconvenience quite so much urine as it used to do in younger life, but the obstruction never becomes serious, complications never set in, and the patient is never troubled by it.

(b). In another class the result is not so good. The balance between work and strength is lost. In some of these the obstruction becomes too great. The physical conditions at the neck of the bladder grow worse and worse; the orifice is raised high above the level of the base, or the posterior wall of the urethra grows forward and blocks the channel. In others the power of the bladder fails. The muscular coat never becomes hypertrophied, or if its strength does increase at first it is only for a time. In many cases both these conditions are present. The bladder becomes unable to expel the whole of its contents. A certain amount of urine always remains behind, occupying the hollow immediately at the back of the trigone, the part that is emptied the last. At first the quantity is not considerable, but gradually, the muscular coat gives way more and more (this part of the bladder has the weakest walls, and owing to its being emptied last has most work to do), and at length a thin walled postprostatic pouch is pushed out. When once a beginning has 
been made further progress is a matter of less difficulty. The conditions that occasioned the dilatation at the commencement have much greater influence over it when it is once established, and the only limit to the size the pouch may reach is the pressure of the other organs around. It has been known to extend down between the prostate and the rectum until it rested upon the perineum.

(c). In other cases the enlargement ends by causing complete retention. The upgrowth acts as a valve, falling over the orifice as soon as the urine presses down upon it, and the more vigorous the effort the more tightly is the valve wedged in. Atony, followed sooner or later by cystitis and decomposition of the urine, is the invariable result. If relief is not given at once, the muscular coat becomes stretched until it loses the power of contracting. If relief is given and a catheter is used, the result is the same, only it follows more gradually. Habitual catheterism ruins the bladder. The muscular coat, relieved of all work, undergoes degeneration, and the bladder is reduced to the condition of a passive receptacle, in which sooner or later cystitis always breaks out. How long it may be before the loss of power caused in this way is complete and final varies very much. Two years, according to Sir Henry Thompson, is enough in any case; but I have known recovery take place after a much longer interval than this. The fact, however, remains, retention caused by a valvular outgrowth always leads to atony, and this as invariably to cystitis and septic urine.

(d.) In a fourth class again the early occurrence of irritability is the prominent feature. The work is increased whether the resistance in front is affected or not. The bladder contracts more and more frequently; the walls grow thicker and stronger; the muscular bundles increase in size and strength, standing out in great columns under the mucous membrane: sometimes a sphincter is developed around the neck, and the cavity diminishes in size. For a time the bladder keeps equal to its work. Then, sooner or later, owing to the persistent straining, chronic congestion and impairment of nutrition follow. Solid œdema sets in. The muscular fibres waste and are replaced by lowly organized fibrous tissue. Some parts of the walls yield and give 
way. Others retain their thickness and grow hard and rigid, and at last the bladder degenerates into a sac of irregular shape, no longer capable of emptying itself, but always retaining a certain amount of urine stagnant in its recesses.

As these different conditions not unfrequently occur together or follow each other, obstruction, for example, increasing until it becomes complete, or irritability suddenly supervening when the bladder can scarcely overcome the resistance, it is easily understood how enlargement of the prostate, acting by itself, without any help from inflammation of the bladder, effects the most profound alterations. It may lead to dilatation or contraction. It may make the walls grow thick or thin, or thick in one part and thin in another. It may alter the shape completely, causing great pouches to protrude. It may leave the mucous membrane smooth and unaltered in colour, or irregularly thickened and covered with depressions. It may cause hypertrophy of the muscular coat, or fatty degeneration, or first one and then the other, and with the aid of old age and chronic congestion it may lead to such a condition of fibroid induration that the walls become hard and rigid, much thicker than natural, and lose their flexibility completely.

When inflammation sets in and the effects that it produces are added to those already described, the condition of the bladder becomes infinitely worse.

Enlargement of the prostate, as already mentioned, is not in any sense of the term inflammatory. There is no connection between it and inflammation; but sooner or later inflammation always does set in, beginning in the mucous membrane of the bladder, and extending thence into the deeper layers and into the substance of the prostate.

There is everything to encourage it. The tissues are badly nourished and unable to resist injurious influences, owing to the state of chronic congestion in which they are living. The epithelial lining can no longer act as a protection against infective organisms. Urine collects and lies stagnant in one part of the bladder. Foreign bodies, in the shape of catheters, are continually being introduced, and though the greatest care may be taken, septic organisms are always being introduced with them. 
Even when the inflammation is transient, it makes all the consequences of the prostatic enlargement much more serious. The mucous membrane becomes more swollen and irritable. The contraction of the bladder is more frequent. The congestion and œdema of its walls grow worse. The nutrition suffers more. The tendency to hæmorrhage is greater. The work is increased in every way and the strength to do it reduced more and more.

The organism that causes cystitis more frequently than any other is the bacterium pyogenes of Clado and Albarran. Achard and Renault (Compt. Rend. de la Société de Biologie, I89I, p. 830) have shown that this is the same as the bacillus coli, which varies to such an extent in the intensity of its action. So long as the mucous lining of the bladder is healthy it is harmless. If, however, the walls become congested, or if retention sets in, the bacillus, which, unless the greatest precautions are taken, is certain sooner or later to gain access by means of the catheter (and may without), breaks through the ill-developed epithelial layer, spreads into the mucous and submucous tissue, and sets up the most intense inflammation.

Septic inflammation of the bladder, under ordinary conditions, without any enlargement of the prostate, is always serious. If the attack is acute a fatal result may ensue in the course of a few days from uræmia and septic absorption. Even when not sufficiently severe for this, the bladder, and, later, the kidneys, sustain an amount of injury that in many cases can never be repaired. The remaining epithelium is detached in flakes and masses. The mucous membrane becomes more and more congested and swollen. In some places the surface is coated with a slimy mixture of phosphates and pus; in others it is reddened or blackened by hæmorrhages of different ages; in others, again, it is. raw and ulcerated, so that it bleeds at the slightest touch. Underneath this the submucous tissue is engorged with lymph and filled with thrombosed veins. Many of the muscular fibres disappear, and are replaced by dense fibroid tissue; and this, in course of time, if the inflammation continues, collects into great ridges, separated from each other by depressions that are often the mouths of sacculi. Every- 
where the pathogenic organisms multiply more and more quickly as the resistance of the living tissues diminishes; and the normal structures are either destroyed altogether and replaced by masses of exudation or so far disorganised as to be incapable of working.

These are the results that ensue when septic cystitis occurs without any obstruction to the exit of the poisonous urine. When the prostate is enlarged, so that the bladder is unable to empty itself, the consequences are, if possible, worse still. The residual urine becomes converted into an irritant poison of the most virulent type. Every drop as it falls from the ureters passed through a similar change, and adds to the amount. The bladder is entirely unable to help itself. Unless some artificial exit is provided, its walls, already weakened and inflamed, are constantly bathed with a poison which increases in amount and in intensity the bonger it is left; and in a very little while, if the patient survives, they are reduced to a condition from which thorough recovery is. almost impossible.

\section{The Effect of Enlargement of the Prostate upon} THE KIDNEYS.

The influence of enlargement of the prostate is not confined to the bladder. The kidneys soon begin to feel it as well; the size diminishes; the pelvis and the calyces become dilated: the capsule grows adherent, so that it cannot be stripped off without bringing away little fragments with it; the delicate connective tissue that lies between the tubules is replaced by a tough fibrous substance; many of the secreting tubules disappear, and the general texture becomes dense and hard.

Changes of this kind are found in most cases of enlargement of the prostate in which there is residual urine, if not in all. Similar changes, more severe in character, are found in general atheroma. The important point is to determine in each case how far they are of constitutional and how far of local origin.

According to Guyon the cause is always and wholly con- 
stitutional. Renal degeneration is merely one part of a general disorder of which prostatic enlargement is another. The enlargement has no direct influence upon the kidneys.

I have shown this to be incorrect in the case of the bladder, and there can be no doubt that it is equally incorrect in the case of the kidneys. That renal degeneration is the rule in general atheroma, and that this is of common occurrence at the same time of life as prostatic enlargement there can be no question. But it does not follow that atheroma is always the cause of the renal changes, much less that it is the sole cause. They may be and often are produced in other ways. It is by no means uncommon to meet with serious renal inefficiency in patients suffering from enlargement of the prostate, whose heart and arteries, so far as they are accessible, afford no evidence of the existence of atheroma.

Increase in the pressure in the urinary passages, for example, if it is kept up for any length of time, leads to very serious changes in the structure of the kidney. Undue frequency of micturition in the face of increased resistance, a condition that is almost invariable in enlargement of the prostate, is sufficient. Dilatation of the pelvis, with well marked atrophy of the secreting portion of the kidney and increase in the amount of interstitial tissue, has been found in cases in which the obstruction was no greater and no nearer than that produced by a contracted prepuce.

A condition almost the same, differing only in the fact that the increase in the amount of connective tissue is relatively greater, is caused according to Albarran ("Etude sur le rein des urinaires") by the action of pyogenic organisms, which are either not sufficiently active to excite suppuration, or else have just succeeded in causing it, and then have succumbed without being able to do any further harm. Positive evidence on such a point, in cases from which the influence of all other causes can be excluded, is naturally difficult to obtain. But if the possibility of the microbic origin of one form of renal sclerosis is admitted (and there is a great deal in favour of it) there can be no question that all the conditions necessary for its development are present in abundance in cases of enlargement of the prostate, especially in those in 
which catheterism is needed to prevent the collection of residual urine.

Another cause for renal degeneration in these cases, and one to which considerable importance has been attached, is the continued congestion of the kidneys which is kept up by the peculiar irritability of the neck of the bladder. The vasomotor nerves of the kidney are affected by everything that irritates the mucous membrane of the deep part of the urethra and neck of the bladder. The passage of a catheter, for instance, is said always to cause a certain amount of renal flushing. As a rule it is transient and quickly passes off, but it has been known to cause renal hæmaturia. When the prostate is enlarged the sensitiveness of the mucous.membrane at the neck of the bladder is often intensely acute. The least irritation is followed by an effect that appears to be altogether disproportionate; and this if constantly repeated many times a day for years together may very well give rise to changes in the kidney which at last are permanent. Those in whom the frequent passage of a catheter renders the mucous membrane of the urethra hard and callous may escape. In others the renal congestion becomes chronic, and at length is followed by contraction and degeneration. Renal degeneration is rarely absent if there is much residual urine.

When due to general atheroma renal degeneration no doubt is more extensive and perhaps advances more rapidly; but I am convinced that although its influence may not be so powerful, enlargement of the prostate, partly by the mechanical resistance that it offers to the descent of the urine, and the encouragement it gives to the growth of septic organisms, partly by the long continued reflex congestion that it maintains, is the actual cause, in a large proportion of cases, of the degenerative changes in the kidneys that have hitherto been laid to the credit, almost exclusively, of age and atheroma.

In the later stages of the disease, when septic inflammation has broken out in the bladder and spread up the ureters to the pelvis of the kidneys, the changes are much more extensive. The walls of the pelvis become swollen and thickened. Abscesses form in them here and there. The surface is 
destroyed by ulceration. The projecting portions of the pyramids are eaten away, and the cavity is filled with an intensely offensive mixture of decomposing urine and pus. The kidney itself soon becomes riddled with abscesses, the pathogenic organisms spreading along the lymphatics and under the capsule, so that the cortex is involved as much as the medulla. The congestion becomes greater and greater; and in a little while, partly owing to the dense and unyielding transformation that the organ has already undergone, partly owing to the actual-destruction of tubules and glomeruli, the secretion of urine begins to fail. At first the quantity may keep up, or may even increase; but inevitably, as these changes advance, the specific gravity begins to fall; less and less of the solid constituents of the urine are discharged, and at last partial suppression follows. 


\section{CHAPTER V.}

The Symptoms of Prostatic Enlargement.

Decrease in the Force of the Stream-Difficulty in Starting-Dribbling at the End-Intermittent Mictarition-Residual Urine-OverflowInvoluntary Escape of Urine-Incontinence-Retention-Increased Frequency of Micturition-Impairment of Sexual Power-PainRectal Symptoms-Alteration in the Character of the Urine-Influence on Health.

IN many patients enlargement of the prostate never causes any symptoms. They live their lives through, even to advanced old age, without suffering a moment's inconvenience from it or being aware of its existence. The increase is so slow that it does not stretch the fibrous capsule and cause pain like malignant disease or inflammation. There is no obstruction, or only so much as the bladder can overcome without difficulty. There is therefore no straining, or congestion of the mucous membrane that lines the neck; and there is no irritability of the bladder or impairment of general health.

- In others, the minority, it is true, but a large one, certain symptoms very soon make their appearance. Some of them are due to the mechanical impediment at the neck of the bladder, caused by the alteration in the shape and size of the gland and the swelling of the mucous membrane. Others arise from the intense hyperæsthesia of the congested or inflamed mucous lining; and others again are secondary, to the consequences induced by these.

Decrease in the force with which the urine is ejected is one of the earliest. The stream flows away from the meatus, dropping vertically down, and no amount of straining is of the least use. Sometimes indeed it makes matters worse, the stream becoming feebler and smaller or stopping altogether for a while, and then beginning again when the contraction around the orifice relaxes. 
Difficulty in starting the strean is another, due to the alteration in the mutual relations of the structures that form the neck of the bladder. Normally as micturition begins the orifice dilates, and the neck becomes funnel-shaped. The contraction of the bladder begins with the longitudinal fibres and those circular ones that surround its main body; at the neck everything is relaxed. When there is a prostatic upgrowth in the uretlira all this is changed; the walls are dense and rigid; the longitudinal fibres cannot shorten the neck or smooth down the projection caused by the apex of the trigone, and the first portion of the urethra cannot dilate and form a single cavity with the bladder. Instead of its being filled with urine ready to pass out, the urine has to be forced down it by muscles that are placed at a disadiantage and soon become tired. All this takes time, and stronger impulses must be sent down the nerves before the bladder can gather up sufficient force.

Dribbling at the end of micturition is a third sign. It usually means that the bladder is beginning to fail. The clean expulsion of the last few drops of urine is mainly a voluntary act. It commences with the involuntary muscle that surrounds the lower part of the bladder, passes on from there to the first portion of the urethra, and is finished by the voluntary muscles that surround the apex of the prostate and the canal beyond. In many diseases of the central nervous system it is imperfectly carried out; and so it may be after exposure to cold. In this case the fault is with the voluntary muscles which do not act aright. In enlargement of the prostate it arises from a different cause. The urine towards the end of micturition is not sent down into the prostatic urethra with sufficient regularity or in sufficient quantity. The voluntary muscles cannot expel it as they should; their power is not impaired, but they do not receive the urine into their grasp at the proper moment.

Intermittent micturition, or sudden stoppage of a full stream without previous slackening (which must be clearly distinguished from simple increased frequency), is not of common occurrence. It can only take place when the prostate has grown so that it can close the passage like a valve. The growth may be confined to the urethra, or it may jut out into 
the bladder from the median or lateral lobes, or from all three together, but it must be in such a position that it can close the orifice when the muscles contract, and leave it free again when they relax.

Residual urine. - In the majority of cases of enlargement of the prostate the bladder after a time fails to empty itself: a certain amount of what is called residual urine is habitually left behind. There may be only a few drops, or there may be niany ounces, thirty and even forty. The quantity in each case varies a good deal from day to day, being greatly influenced by nervousness, the state of health of the patient at the time, and other causes, but throughout there is a continuous, if fitful, tendency to increase. The patient, unless it has actually been shown him already, is hardly ever aware of it. If the quantity is small there is no means of his knowing it. If it is large it diminishes the carrying capacity of the bladder by so much, and leads to frequent micturition, not unnaturally conveying to his mind the conviction that he is passing urine too frequently, or in too great a quantity; never that he is constantly retaining some.

The diagnosis is easily made by passing a catheter after the patient has, as he imagines, emptied his bladder; and no examination of any case of enlargement of the prostate is complete without this. The precautions that must be taken will be detailed later, but it may be mentioned here that the proceeding, especially if the amount is large, is by no means devoid of risk. To form a correct estimate of the quantity, this must be repeated three or four times under different conditions. The amount is liable to vary, and careful note should be taken of any difference that is found. If it is at all considerable it is an indication not only that part of the muscular coat has lost its power of contracting, but that a further portion is almost in the same plight.

Residual urine is not peculiar to enlargement of the prostate. It is met with in stricture and other conditions as well. Wherever it occurs the immediate cause is failure in the muscular power: the work is too great. It may be simply due to fatigue; the muscles begin well, but become tired out before their task is finished. This is especially likely to occur in old people, and in those in whom the nutrition of the coats 
of the bladder has been impaired by long continued congestion. Or it may be due to the prostatic growth in the urethra acting as a valve. When the muscular fibres that surround the lower part of the bladder begin to contract, the orifice is closed before the cavity is emptied, and some of the contents are left behind. Or it may be due to both of these causes.

The urine of course collects in the lowest part of the bladder. This is no longer the region of the orifice, for this has been raised by the elongation of the urethra, but the thin-walled post-prostatic portion. Here it rests almost undisturbed in all the ordinary positions of the body, standing, sitting, or lying down. As the quantity increases this part of the bladder is pushed out into a pouch, which may stretch down between the rectum and the prostate until it reaches the perineum.

This portion of the bladder-wall yields the first, partly because it is weaker and thinner than the rest, partly because the raising of the outlet places it in a position of peculiar disadvantage. As the end of micturition approaches pressure is brought to bear from all sides upon the fluid that is left. If the orifice is open the resistance is least at that spot; the urine flows out and the pressure is relieved. If it is rigid, or, still more, if it is closed by a valvular outgrowth, the weakest part of the bladder-wall ceases to contract the first, and then begins to yield and stretch under the pressure thrown upon it by the rest. For this reason when once there is a commencement of residual urine, more or less rapid increase is invariable. In old standing cases the size of the post-prostatic pouch is only limited by the pressure of the organs around it.

The fact that the urine is retained in the bladder does not in any way affect its character so long as the mucous membrane remains healthy and no-irritant reaches it. The residual amount is mixed with that which comes down from the ureters and is gradually changed. But as the bladder always exerts a certain amount of tonic contraction upon the fluid inside it and (unless the residual urine is suddenly drawn off) is never too large for its contents, these remain almost stationary. As a consequence, when the mucous membrane becomes congested or cystitis sets in, and a large amount of 
epithelial débris or of pus is added to the urine, this, being heavier, slowly settles down through the layer of urine at the bottom of the pouch and remains stagnant there.

Overflow. - As the amount of residual urine constantly tends to increase, a time at length is reached at which the potential capacity of the bladder is greatly diminished. Micturition in consequence becomes more frequent and at last almost continuous. The bladder is as full as it can be; its walls are so. tensely stretched that it can hold no more, and as the urine enters it by the ureters some must leave it by the urethra. This is known as overflow, and must be clearly distinguished from incontinence.

Involuntary escape of urine at night is the first sign of this. Overflow cannot take place so long as the external sphincter is in active work. It begins, therefore, at night during sleep. Later it takes place during the day as well, especially when the abdominal pressure is raised by muscular exertion; the sphincter or the nerve centre that controls it is either tired out or taken unawares.

Incontinence.-This is one of the rarest of the symptoms of enlargement of the prostate. Formerly it was often confused with overflow, but as Sir H. Thompson has shown, the two conditions are entirely distinct. In true incontinence the bladder is empty and remains so; in what is commonly called incontinence, but which is really overflow, the bladder is over full and continues over full throughout.

Enlargement of the prostate can only cause incontinence of urine when the growth at the neck of the bladder is of such a shape that the urine flows continuously into the prostatic urethra; and when, in addition to this, the sphincter at the apex of the gland is unable to act. Either of these conditions alone is insufficient; they must be present together.

The first is not uncommon. . It is by no means rare to find on passing a catheter that the prostatic urethra is dilated into a chamber lying in front of the bladder. Sometimes it is so large that a short beaked sound can rotate in it without difficulty. The orifice by which it communicates with the bladder may be the widest portion of the cavity, so that it is funnel-shaped with the apex downwards, or it may be quite small. The essential point is that the communication 
is kept open. The urine is only prevented from flowing away by the contraction of the voluntary sphincter in front, and the resistance of the urethra. The enlargement in cases of this kind is, generally speaking, uniform. All parts of the gland are involved pretty nearly equally. Mercier, in his four cases, described the median lobe growing forward, like a wedge, separating the lateral ones, and opening up the neck of the bladder. As Sir H. Thompson points out, the wedge in these cases is usually directed upwards towards the bladder, and not forwards. Nor is this action necessary so long as the median portion of the gland is sufficiently wide; and such a condition is not very rare.

Loss of power over the external sphincter, the other factor necessary to the production of true incontinence, is only met with in affections of the central nervous system, or of the nerves themselves. I have met with it, for example, in cases of locomotor ataxy and in cerebral softening; and in one of these it was associated with this particular form of prostatic enlargement, and with true incontinence. The bladder which, when I first saw the patient only a few weeks before, had been of very large capacity, could only retain a few ounces. As soon as more than this collected it began to flow away. The only forces left in action were the tonic contraction of the muscular coat, which was not very strong, and the resistance of the urethra. As soon as the one exceeded the other incontinence resulted; and had the tone of the bladder been good it would have remained persistently empty.

Retention of urine.-Complete retention may occur without warning or anything that could raise a suspicion of enlargement. Much more frequently there has been trouble for a long time before, such as delay at the beginning, want of force in the stream, or some other of the symptoms already mentioned. The imniediate cause is either sudden failure in power, or sudden increase in the resistance, or both together.

The former is seldom altogether wanting, although it is rarely entirely sufficient by itself. Occasionally, however, retention follows voluntary postponement of the act of micturition. Urine is allowed to collect in the bladder until the usual quantity is exceeded. The muscular coat has been 
weakened by congestion or inflammation, but is still able to deal with a moderate amount. Suddenly it is called upon for a much greater effort, and it fails.

Sudden increase in the resistance is more common. It arises nearly always from congestion and swelling at the neck of the bladder. There may be a single valvular outgrowth langing over the orifice, or there may be three meeting over it when micturition begins; or there may be merely enlargement of the lateral lobes compressing the urethra from side to side. For some time past the bladder has been acting at a disadvantage, but has been able to get rid of its contents. Suddenly the mucous membrane becomes congested and swollen; the size of the valve is increased, or the half-closed passage is completely shut, and nothing more can pass.

Patients with enlargement of the prostate are peculiarly susceptible to these attacks of congestion, especially when the kidneys have be̊come affected. Or perhaps the true explanation is that congestion at the neck of the bladder is more common in old men than is usually imagined, but it does not cause retention or any other trouble unless it is superadded to already existing enlargement. Whichever of the two is correct, in retention of urine due to enlargement of the prostate congestion is almost invariable.

The congestion may be inflammatory, arising from cold or from an irritating condition of the urine, or it may be passive, the result of obstruction to the return circulation. Excess in food or drink, especially in alcohol ; exposure to cold or wet; attacks of constipation; sexual indulgence; riding; long railway journeys; anything in short that increases the amount of blood flowing into the pelvic organs may act as the exciting cause; and when it has once set in the straining that attends the act of micturition makes it tenfold worse. Often the veins in the mucous membrane become so distended that they give way, and the blood pours out either into the thickness of the wall or into the interior of the bladder, where it mixes with the urine.

The relative share taken by glandular overgrowth and by congestion in causing prostatic retention can only be estimated in any individual case by the events that follow. Simple congestion, if the urine is drawn off at regular inter- 
vals, and the patient is kept quietly in bed, without stimulants and secure from all sources of irritation, soon begins to subside. Even when thrombosis has occurred, a certain degree of improvement usually shows itself in the course of two or three days, although it is often many weeks before control becomes as good as it was. Enlargement, on the other hand, remains unaffected, or slowly increases.

Retention must be distinguished from the accumulation of residual urine. In the former the exit is closed, and immediate relief is imperative. In the latter the surplus flows away without hindrance, and although it is not a condition that should be allowed to continue longer than can be helped, there is not the same demand for urgency. If retention is not relieved at once the pain becomes more and more severe; the bladder and then the ureters and the pelvis of the kidneys are distended to their utmost; the secretion of urine is checked by the rising pressure, and symptoms of urinary poisoning rapidly follow.

Even when the urine is drawn off with the least possible delay, retention always inflicts a certain amount of injury upon the urinary organs. The muscular coat of the bladder is strained and loses power, especially if the walls are thin and the cavity large. For weeks after an attack of retention the residual urine is more abundant than it was before; and even complete atony may occur. Nor do the kidneys escape; fever always follows acute retention, and it not unfrequently happens that it is some considerable time before the secretion of urine becomes normal again.

Increased frequency of micturition begins very early, long before there is any considerable amount of residual urine, and is due to the irritability of the mucous membrane lining the neck of the bladder and the first part of the urethra.

The stimuli that excite micturition always start from this part, the rest of the bladder being comparatively insensitive. Morbid growths, for example, situated in this part, or calculi impacted there, act as intense irritants, while in other parts of the bladder they have but little effect; and bulbous sounds while passing through the orifice often excite a keen desire to urinate, which ceases as soon as the bladder is reached. In enlargement of the prostate, this part is very liable to become 
congested; the mucous membrane lining it grows exceedingly sensitive, and causes that would ordinarily have very little influence, act with the greatest vigour.

Great increase in the frequency of micturition during the early stages of the disease usually means that there is some irritant present, such as a growth springing up in the neck of the bladder or some morbid condition of the urine. Later, when the mucous membrane is inflamed, the slightest stimulus is sufficient, the mere contact of the urine with the tender surface or the pressure caused by spasmodic contraction of the muscles. When this stage is reached the frequency becomes greater and greater, until at last micturition is almost incessant, and causes the most acute suffering. The muscle around the neck of the bladder is in a state of constant spasm. The mucous lining, which is so tender that it cannot bear the slightest touch, is crushed by its contraction, and the burning pain at the neck of the bladder never ceases. The only time when there is the least relief is the moment that the urine is actually passing.

The worst cases are those in wlich a minute ulcer or fissure is formed between the congested folds at the neck, exposing some of the terminal nerve filaments. Every few minutes some drops of urine are ejected spasmodically, and the moment micturition is over the intense irresistible desire begins again, worse, if possible, than it was before, until the patient's strength and endurance are worn out.

In many cases of enlargement of the prostate the quantity of urine that is secreted within twenty-four hours is considerably increased, and this naturally has a direct, though not very important, influence upon the frequency of micturition. The changes in the kidney that lead to it may be the result of advancing age, or of backward urinary pressure, or of commencing atheroma, or of chronic congestion set up by reflex irritation from the neck of the bladder, or of all of these acting together. The specific gravity of the urine is lowered at the same time, and this, again, when the mucous membrane is so exceedingly sensitive, is another cause especially as the urine secreted under these conditions not unfrequently contains minute crystals of oxalate of lime.

It is usually said that the frequency is greater during the 
night time than during the day. This does occur sometimes, but I am inclined to doubt whether it is so common as is usually believed. Careful inquiry into cases that give this history often shows that the patient passes water much more frequently than he imagines. There is nothing during the daytime to impress the act of micturition upon his mind, so long, that is to say, as there is no difficulty. The conveniences are so great that they are taken advantage of almost unconsciously - at any rate, without sufficient mental effort to ensure the number of times being remembered unless every single thing done during the day is passed under review. Micturition has become an acquired automatic act. At night it is different, and until habit has become second nature, or the number of times is considerable, each one is recollected and counted.

The time when the increased frequency occurs is generally the early morning. The patient sleeps fairly well for the first few hours after going to bed, a longer interval without micturition than is usual with him during the daytine. At last the call becomes so urgent that it wakes him. By that time the bladder is uncomfortably distended; the muscular coat is stretched beyond its custom; the work it is called upon to perform is greater than usual, and it becomes tired before its task is finished, so that the patient has to rise two or three times again before morning. The first interval was too long.

In a few cases, however, the frequency of micturition during the night is certainly greater, and the cause, I believe, is to be found in the character of the urine. There is nothing in the recumbent position to occasion it, nor, as a matter of fact, is it noted when the patient is lying down in the daytime. It cannot be assigned to congestion of the sexual organs (such as not unfrequently comes on during the nighttime in cases of prostatic enlargement), except in the most distant way, by increasing the amount of blood flowing through the pelvis. Penile erections, as a rule, exert an inhibitory influence upon micturition. On the other hand, there is no doubt that late-dinners, and particularly the common habit of taking spirits the last thing at night, augment the quantity of the urine secreted during the next few hours, and frequently render it more irritating in quality. 
Under normal conditions the cause may be too slight to produce any effect, but when the neck of the bladder is congested and unduly irritable exceedingly little is required. Whether this is the general reason or not I am not prepared to say; certainly it was the cause in some of the cases that have come under my notice, for by careful attention to particulars of this kind, and by relieving the congestion as far as possible, I have not unfrequently succeeded in prolonging the intervals of rest very considerably.

It is probable that the greater frequency at night would never have attracted so much attention had it not been for the striking contrast presented in this respect by patients who are suffering from stone in the bladder. Under these circumstances the frequency of micturition is very much greater during the day. At night, and when the patient is lying down, so that the calculus does not move or come into contact with the neck of the bladder, it subsides almost entirely.

In the later stages of the disease, when the muscular fibre of the bladder has lost, or almost lost the power of contraction, frequency of micturition may also be caused by the accumulation of residual urine; but this cannot occur until the amount is considerable. "If a bladder, which holds ten ounces comfortably, fails to evacuate the last ounce of water, the only effect of this is to reduce the comfortable capacity of the viscus to nine ounces, thus necessitating an increase in the number of urinations not sufficient to be appreciated and not commensurate with the frequency noted in many cases where the residual urine is small in quantity. That residual urine alone, without the supervention of congestion or inflammation of the vesical mucous membrane, is not sufficient to cause frequent urination, is shown by the fact that a healthy man may voluntarily retain in his bladder portions of the urine at each micturition and yet will suffer no appreciable increase in diurnal number of micturitions." (White).

Impairment of sexual powier. - Whether enlargement of the prostate has any direct influence upon the sexual power is uncertain. As already mentioned, the prostatic fluid appears to be essential to the continued activity of the spermatozoa; 
and certainly in most cases of enlargement the structure of the whole gland is so affected that it is scarcely probable that its function can be carried out efficiently. It is conceivable that certain forms of enlargement might interfere with the due carrying out of the sexual act itself, but the question is not yet ripe for settlement.

The other symptoms that are met with in cases of prostatic enlargement arise almost entirely from the congestion and cystitis which accompany it.

Pain is never caused by mere enlargement; the rate of increase is too slow. It is always due to congestion or inflammation increasing the sensitiveness of the mucous membrane at the neck of the bladder or stretching the fibrous tissue that surrounds the gland, and is proportionate to the acuteness of the attack. There may be merely a little aching in the perineum, with some increase in the frequency of micturition, or there may be intense pain shooting down the thighs into the testes, or round the back, down, in short, every branch of the sacral plexus, with burning at the neck of the bladder and at the end of the penis so intolerable that the patient can hardly be held responsible for his actions.

In one case under my care, in which the amount of residual urine was very large, the symptom of which the patient complained most, and for which he consulted me, was pain along the course of the two great sciatic nerves, but especially the left one. It was caused, there was no doubt, by the weight of the fluid pressing upon the sacral plexuses, for it was relieved immediately when the quantity was reduced; but such a condition is exceptional.

Rectal symptoms. - A sense of fulness in the rectum, as if there was something there that could not pass, is a common cause of complaint. The patient is always desiring to go to stool and perpetually straining without result. In a few cases this arises from direct pressure, the gland attaining an enormous size or growing backwards to an unusual degree. Instances have been known in which it has proved an actual source of difficulty in this way. But a far more frequent reason is the congestion of the mucous lining, arising from the constant straining. In the later stages hæmorrhoids and sometimes prolapse make their appearance, and as the power 
of control diminishes, involuntary escape of flatus and fæces during micturition becomes of common occurrence, and a source of great distress.

The chavacter of the urine.-So long as the bladder remains healthy the most important change is an increase in the quantity and a diminution in the specific gravity. This is due to the fibroid induration and contraction of the kidneys which is so commonly present in long-standing cases of enlargement, and which is the product of many different causes acting together-age, arterial degeneration, the strain thrown upon the secreting structure by the backward pressure from the bladder, and the persistent hyperæmia of the kidneys kept up by reflex irritation from the over-sensitive neck of the bladder.

In several instances of definite, but not very great, enlargement of the prostate, I have met with curiously sudden changes in the character of the urine. Within half an hour there may be a difference of many degrees in the specific gravity, independently of the quantity or the quality of the fluid consumed. The urine may be almost colourless, and of very low specific gravity; and then, in a few minutes, become much darker, and contain a fair proportion of solids. Often too there is a trace of albumen. I believe these sudden changes are due to the action of the nerves of the kidney, which in such cases are kept in a constant state of irritability by stimuli from the intensely sensitive mucous membrane at the neck of the bladder. Whatever the cause, these rapid fluctuations emphasize very strongly the necessity for repeatedly examining the urine in all cases of prostatic enlargement before forming a final conclusion as to the condition of the kidneys. In all the cases under my care in which this variability occurred, there was good reason to suspect that a certain degree of contraction was present already.

In the early stages of the disease albumen and casts are seldom found. But if, when the specific gravity of the urine is habitually low, there is the least disturbance, such for example as that induced by passing a catheter and drawing off the residual urine, they make their appearance at once.

In the bladder the urine in uncomplicated cases undergoes but little alteration. There is a more rapid proliferation of 
the epithelial lining; many of the cells are detached, and these mixed with leucocytes form a thin floating cloud; but nothing more. The least degree of congestion produces a marked change at once. The number of leucocytes increases; there is no longer a faint cloud but a distinct deposit that collects at the bottom, and blood is often present. Occasionally there is free bleeding, a varicose vein at the neck of the bladder giving way, or more commonly, some injury being inflicted by the catheter. For a day or two the colour is bright red, then it becomes brown and smoky, growing lighter and lighter until it is normal again. In exceptional cases the hæmorrhage is so free that the bladder becomes distended with coagula, which if not broken up and removed slowly melt down into a dark slimy mass, coming away only with difficulty and after many days.

Other and more serious changes follow the entrance of septic organisms. The mucous membrane that lines the ureters and the pelvis of the kidneys, and then the tubules themselves become involved. Albumen and casts make their appearance in abundance. The quantity diminishes; the reaction becomes alkaline: the odour most unpleasant, and all the phosphates and salts that are insoluble in an ammoniacal fluid are precipitated in the deposit that covers the inflamed and often ulcerated surface. In the bladder the decomposition advances farther still. The mucous membrane in a state of acute inflammation pours out a quantity of pus which mixes with the phosphates, and acted upon by the alkalies forms a tenacious glairy mass that separates from the rest and clings to the side. The odour becomes peculiarly offensive, owing in all probability to the bacillus coli, the ordinary organism of suppurative cystitis; and the irritation so intense that the bladder is forced to expel its contents every few minutes with the most painful spasm, crushing together the raw and ulcerated surface at the neck.

The state of health.-Enlargement of the prostate has no influence upon the general health, except through the secondary changes it induces in the kidneys and the bladder. In the early stages there is nothing to notice. As micturition becomes more frequent, and an undue strain is thrown upon the secreting structure of the kidncy, vague evidence of 
failing strength begins to appear. The patient seems to have aged rather suddenly. His friends describe him as not the man he was. $\mathrm{He}$ is more liable to colds and slight feverish attacks, and he does not rally from them at once. There is not the same amount of energy in what he does; neuralgia is common ; sleep is broken, and the appetite capricious. Loss of weight is very general; the skin feels dry and harsh, and wrinkles become much more evident, especially around the eyes. But with all this the patient may complain of nothing very definite; the failure is general, and the strength and nutrition of all the tissues suffer.

Events move much more rapidly when acute congestion sets in and the neck of the bladder becomes irritable. The kidneys are congested, their secreting power is impaired, and the tissues cannot dispose of their nitrogenous waste. The loss of flesh and vigour rapidly increases, and the constant pain at the neck of the bladder and the ceaseless desire to urinate undermined the patient's strength completely. The bowels become disordered; the perpetual straining leads to hæmorrhoids, which often bleed profusely; the appetite is lost; the tongue becomes dry and red; sickness is not uncommon; and all the signs of incipient uræmia make their appearance.

When cystitis and pyelonephritis set in septic absorption is added to the rest. The pulse becomes small, quick, and feeble: the temperature rises to $102^{\circ}$ or $103^{\circ} \mathrm{F}$.; rigors sometimes occur ; and the patient passes into a dreamy state, with low muttering delirium, until diarrhœa with a subnormal temperature or coma, and perhaps at length convulsions follow. 


\section{CHAPTER VI.}

Diagnosis.

The Condition of the Prostate-Rectal Examination-Urethral Examination-The Length of the Prostatic Urethra-Its Direction-The Shape of the Orifice-The State of the Bladder-The Amount of Residual Urine-The Tone of the Muscular Coat-The Condition of the Mucous Membrane-The Character of the Urine-The Condition of the Kidneys.

THE diagnosis of enlargement of the prostate does not appear to present much difficulty. Diminution in the force with which the stream of urine is propelled, and increased frequency of micturition, especially during the night and in the early morning, occurring in a man past middle age, suggest it at once. But a diagnosis distinguishing with certainty this form of enlargement from other diseases, some of which are attended by an increase in the size of the gland, can only be made by careful rectal and urethral examination.

Stricture is met with at all ages, but it is much more common before middle life. When an instrument is passed down the urethra the obstruction is in the penile or the membranous portion, in front, therefore, of the prostate. Organic stricture is unknown in the prostatic portion.

$A$ calculus if it can move freely in the bladder is attended by symptoms that bear but little resemblance to those present in enlargement of the prostate. There is no failure in the force of the stream. The frequency of micturition is greater during the daytime, and is increased by movement; hæmorrhage is of common occurrence, especially after exercise, but it follows the stream of urine; and pain is felt chiefly at the end of the penis. But it must not be forgotten that in many cases of enlarged prostate there is a calculus lying in the postprostatic pouch, and giving rise to none of these symptoms. Sometimes it is movable though it may not leave the recess 
in which it lies. Often it is firmly fixed either by its shape, or by the contraction of the fibres of the posterior wall of the bladder which hold it against the prostate. This is very much more common than is usually believed, and in every case of obstinate cystitis associated with enlarged prostate, special examination of this part of the bladder should be made with a long, sharply curved sound, the beak of which can dip behind the gland. If this is not perfectly satisfactory an $\mathrm{X}$ ray photograph should be taken of the bladder. I have on several occasions detected calculi in this way which had eluded every other method of investigation.

In one case under my care some difficulty was caused by the presence of a calculus of unusual size, fixed in but projecting out of the lower end of the ureter. The rectal surface of the prostate was enlarged, and, for a time, the calculus was taken to be a pedunculated outgrowth almost distinct from the main body of the gland.

Malignant disease of the prostate, in its earlier stages, is very difficult to distinguish from simple enlargement. The most characteristic symptom is persistent hæmorrhage. Bleeding from the prostatic urethra is of frequent occurrence in ordinary enlargement, but nearly always some direct exciting cause can be found, such as an attack of congestion, or the passage of an instrument. Hæmorrhage, apparently without cause, and of frequent occurrence, should always excite suspicion. In doubtful cases it is possible that the microscopic examination might reveal the presence of cancer cells. Afterwards, in the later stage of the disease, the rapidity with which the gland increases in size, the intense pain and the profuse hæmorrhage are characteristic.

Chronic inflammation of the prostate, when it begins about middle life, is sometimes confused with the early stages of true enlargement. The difficulty is the greater as the two may occur together, either the prostate after having been the seat of chronic inflammation for years, becoming enlarged as age creeps on ; or a gland that is enlarged already, becoming inflamed. Sometimes the inflammation begins on the mucous surface, from rough usage of instruments or from septic or specific urethritis; sometimes, as in cases in which thrombosis is a prominent feature, it starts in the periprostatic 
tissues. The history, and the examination under the microscope of the first few drops of the urine that are passed, afford the best means of judging. Frequency of micturition and slight hæmorrhage are common to both; but in enlargement the bleeding usually precedes or accompanies the flow of urine, while in chronic prostatitis it occurs towards the end. In simple enlargement there should be no tenderness on rectal or urethral examination. Pain at the end of micturition points either to inflammation or the presence of some irritant. The difficulty is greatest in cases of early fibroid degeneration of the enlarged gland, when the symptoms are often well marked although the size of the prostate is not materially increased. There is little or no resemblance between the symptoms of chronic prostatitis and those that occur in the rapidly growing adenomatous form of enlargement.

Tubercular disease of the prostate for the most part occurs in young adults, and is accompanied by deposits in the mucous membrane of the bladder, or in the vesiculæ seminales and the epididymis. I have known it, however, late in life, in a case of senile tuberculosis. As a rule there is not much enlargement, and no elongation of the prostatic portion of the urethra. The rectal surface may be very irregular in outline and in consistence. The symptoms rarely attract attention until caseation and softening have set in, and the mucous membrane lining the urethra has given way. Then their intensity leaves no doubt as to the presence of ulceration. If the urine is centrifugalised, tubercle bacilli can generally be detected.

Examination of the Prostate.

At a first examination it is not easy to determine the share taken by the growth, vascular congestion, and chronic inflammation respectively, in causing the increase in size. The symptoms that attract the patient's attention are nearly always due to the two latter; he seldom applies for relief on account of the growth alone.

Time soon tells. Congestion, if the cause is removed, 
begins to subside at once. Thrombosis, which, judging from the chains of phleboliths so frequently found in the prostatic plexus of old men, must be of common occurrence, takes a little longer. A certain amount of inflammation is always associated with it. The capsule and the loose cellular tissue that holds the veins together are swollen and filled with lymph; and there is pain and tenderness when pressure is made with the finger in the rectum. But in a few days, if thorough rest is enjoined, the thrombi disappear or shrink up, and the exudation around the vessels becomes absorbed. The increase that is due to growth, on the other hand, remains permanent.

It follows that after an attack of acute retention (in which congestion nearly always takes a very important share) no definite opinion as to the size of the prostate or the condition of the muscular tone of the bladder should be given until the effects due to these accessory causes have had time to disappear. Still less should any operation, except one of urgency, be performed.

The alteration in the shape of the prostate is ascertained by rectal and urethral examination separately and in combination.

Rectal examination.-If the patient is lying on his side the posterior surface of the gland can be explored by introducing the finger into the rectum. The size, however, and especially the degree of projection upwards towards the bladder, can be ascertained a great deal better (if the abdomen is not too stout) by placing the patient on his back with the knees well drawn up, and while the forefinger of the right hand is in the rectum, making firm pressure over the pubes with the fingers of the other hand. The bladder should, of course, be emptied first. In thin patients it is quite possible to grasp, and even to manipulate, an enlarged prostate in this way.

In the young adult the prostate does not project backwards into the rectum, though it can be plainly felt through it. The finger can reach above it easily, and explore the trigone, and with more difficulty can pass a little way round on either side. The consistence is uniform and firm, and pressure does not cause pain or any additional discomfort. The upper. border in most cases is slightly notched; definite convexity 
points to commencing enlargement. The lower end can be traced into the membranous part of the urethra.

When the median lobe of the prostate is enlarged, or when all portions are enlarged together, it grows backwards against the anterior wall of the bowel. The finger meets it at once, and has to be directed towards the sacrum in order to pass it. The upper border becomes convex and massive, and the trigone recedes so far that the finger may be quite unable to reach it. When the lateral lobes only are involved the trigone is carried as high, but the backward projection is less, and the notch remains distinct. Any conspicuous inequality between the two sides can be detected easily.

In general enlargement the surface is rounded and uniform. Exceptionally it is nodular with projections upon it slightly softer than the parts between. Examination always causes discomfort, but it should not cause pain. General tenderness. points to recent congestion. When it is most marked upon the sides and the upper part of the gland, and the central portion near the apex is unaffected, there is in addition thrombosis with inflammation of the cellular tissue around the veins. In these cases the increase in size is rapid. The mucous membrane of the rectum is hotter than natural, and. the sphincter is not unfrequently in a state of rigid spasm.

Examination by the rectum, however, gives but a very. limited amount of information. It can tell nothing as to the position occupied by the urethra, or the degree of compression to which it is subjected, or the alterations in its course and in the orifice of the bladder. The prostate, as felt through the rectum, may be normal in size, or so slightly enlarged that it tends rather to throw discredit upon the diagnosis than to assist it, and yet there may be distinct and serious urinary troubles. As McGill pointed out, the prostatic enlargement that causes obstruction is intravesical (and urethral), not rectal. Very small growths in the region of the orifice may cause complete retention, while that part of the body of the gland that can be felt through the rectum is entirely unaffected.

Urethral examination.- Various instruments are required to make this complete. A full-sized prostatic catheter to ascertain the length; a short-beaked metal sound; various soft 
catheters, "coudé" or "bi-coudé," to estimate the degree of projection upon the floor; the cystoscope, and one or more prostatometers to measure the antero-posterior and transverse diameters of the canal. Whether cocaine or a general anæsthetic should be used depends upon the condition of the patient, and the amount of pain caused by examination. The urethra in cases of this kind is as a rule more tolerant than it is in younger men suffering from stricture. The patient should be lying down, in bed if possible, and must be warmly clad. If not accustomed to the passage of instruments, or in any case if the examination is a long one, he should not be allowed to move for some hours afterwards, and especial care must be taken to guard against exposure to cold. A gentle purge given the evening before, to ensure the lower part of the bowel being emptied, tends to diminish the amount of local jrritation.

All instruments must be thoroughly disinfected beforehand by boiling or by a prolonged immersion in some strong antiseptic solution, which is washed off immediately before the instrument is used with water that has been boiled. The hands, the skin of the penis and the prepuce must be thoroughly washed first with soap and water, as in the case of any other surgical operation in which the preservation of asepsis is essential; and then the glans and the meatus must be well cleansed with a solution of corrosive sublimate, one part in five thousand. An irrigating catheter is then to be passed into the fossa navicularis and the front part of the canal washed out from behind with warm boracic lotion; and when this is finished, the catheter is to be pushed on to the deep part of the urethra and the process to be repeated there.

The length of the prostatic portion is measured by means of a catheter. The distance the instrument has to pass before the point enters the membranous part is noted, and that at which the urine begins to escape; the difference corresponds roughly to the length required.

Two points must be remembered in doing this. One is that the measurements are not taken from the same part of the catheter. In a large-sized instrument, such as is usually employed in cases of enlargement of the prostate, there may be more than a cm. difference between the eye and the end. 
The other is that if the prostatic urethra is dilated a few drops of urine always flow from it as soon as the catheter enters the cavity. If the communication with the bladder remains open the whole of the urine can be drawn off in this way. The result, therefore, requires checking before it can be accepted as absolutely correct.

Every form of prostatic enlargement may be accompanied by increase in the length of the prostatic urethra. This measurement, therefore, does not add much to the information obtained by rectal examination. On the other hand, all the symptoms of prostatic obstruction may occur without alteration in the length of the urethra. If the stroma-growth begins early in the course of the disease, before the adenomatous tissue has spread to any extent, the gland increases in density without undergoing any material change in size. In such a case the prostate becomes as hard as scirrhus, and the structures around the neck of the bladder are so rigid as to render the passage of urine exceedingly difficult. In one of these cases McGill was unable to remove a fragment larger than a pea. The mere fact, therefore, that the length remains normal is not to be taken as an indication that the prostate is not affected.

The direction of the urethra is equally important. The curve may be increased or lessened. It may deviate to one side or the other, and it may be uniform throughout, or sharply bent at one point and almost straight in the rest of its course.

Lateral deviation is shown at once by the twisting of the handle of a metal instrument as the point passes by the obstruction. It may be caused either by unequal growth of the lateral lobes, or, if the twisting takes place as the instrument is entering the bladder, by the projection forwards of a median upgrowth in the posterior margin of the vesical orifice. Exceptionally, the urethra is so tortuous from the presence of nodular projections fitting into each other that there is some difficulty in introducing a rigid instrument.

Angular bending is shown by the way in which an ordinary catheter is caught and stopped at a point from two to three $\mathrm{cm}$. beyond the membranous portion, while an elbowed one, or a gum-elastic from which the stilet is withdrawn when the 
oustruction is reached, passes with ease. Bending in this situation is proof of the presence of a growth in the posterior wall of the upper part of the urethra. Increased lengthening at the same time is proof that the upgrowth has raised the neck of the bladder as well, and extends into the orifice.

Further information with regard to this may be obtained by observing the extent to which a metal instrument requires to be depressed between the patient's legs when he is lying down. If the handle of a full-sized prostatic catheter has to be lowered almost to the horizontal before the point enters the bladder the curve of the urethra is not only of exceptional length, but is so directed that the orifice looks towards the pubes, or at least towards the anterior abdominal wall, not upwards as it should. In other words, there is a great upgrowth behind it, and beginning to project over it.

A short-beaked metal sound is of use for ascertaining the presence of a cavity in the prostatic urethra and the shape of the vesical orifice. The curve must be bent so that the tip is at right angles to the shaft, and should not be more than an inch in length. If the walls of the urethra are in contact and there is no cavity the point can only be moved backwards and forwards. In any other direction it meets with an immediate check, and the attempt is stopped by pain. If, on the other hand, there is a cavity, it can be made to rotate from side to side, and move about with freedom. In some cases there is a space capable of holding several drachms of urine.

The shape of the orifice.-Outgrowtis springing up around the orifice of the bladder can be detected in the same way. If the margin is smooth and even the curve of the sound sweeps round it without difficulty. When, on the other hand, it is irregular, with projections here and there, the difference in level is felt immediately. A uniform collar-like upgrowth may be suspected when the length of the urethra is increased while the margin remains level. If rotation from side to side is stopped as the point approaches the median posterior line there is in all probability a single, and perhaps a valve-like upgrowth. A fairly correct estimate as to the size and degree of mobility of an upgrowth of this kind can sometimes be formed by noting the additional length to which the sound 
must be introduced before it can pass over it, and the sharpness of the jerk with which it slips past it.

A valvular outgrowth is to be suspected when the bladder retains its power, but is unable to get rid of its contents, while a catheter passes in without difficulty. The diagnosis is confirmed by introducing a catheter with a terminal orifice down to the apex of the prostate and connecting it by a rubber tube with a funnel containing warm boracic solution. Under normal circumstances a pressure of a few inches is sufficient to make the fluid enter the bladder. Roughly, the measurement may be taken by the height to which it is necessary to raise the funnel. If greater accuracy is desired the tube may be connected with a manometer by means of a side branch. In cases of valvular obstruction, the fluid enters freely at the ordinary pressure. If, on the other hand, retention is due to congestion and compression of the urethra by the lateral lobes it remains stationary until the funnel is six feet or more above the patient's body, and then it only trickles in. I have found this method of use in several instances, in confirming the impression derived from exploration of the orifice with a sound.

By combining rectal with urethral examination a very accurate estimate can be formed of the amount of growth in the median posterior line. The shaft of the instrument can always be felt at the apex of the gland, and if it is reversed, and the parts are normal, the point can be felt equally well in the bladder.

In some cases, growths that surround the orifice can be seen by means of a cystoscope, but so far as enlargement of the prostate is concerned this instrument has not fulfilled the expectations formed of it. In many instances it is very difficult to introduce. It is almost impossible to see with it the bases of the growths. They may be broad or narrow, pedunculated or not; owing to the way in which they fit closely together, only the summits are visible. And it can give no information about growths in the posterior wall of the urethra, or the density that the enlargement has attained. On the other hand, when it can be introduced, it is of considerable service in ascertaining the condition of the mucous membrane of the bladder and the presence of sacculi. 
Another method that I have found of great assistance in forming a diagnosis, is based upon the relation that exists between the size and shape of the prostatic urethra and that of the prostate itself. It is true that this must not be pushed too far. The prostate may be distorted and covered with outgrowths without of necessity entailing much alteration in the channel that runs through it. But leaving on one side cases that present conspicuously exaggerated features, and restricting it to the ordinary type of overgrowth, in which there is an ever increasing difficulty in emptying the bladder, some degree of proportion certainly does exist in the majority. It is true that it is not possible to define accurately in every case the parts that are enlarged or the extent to which they have grown, but it is equally true that great alterations in the dimensions of the urethra are practically always associated with definite changesin the shape and size of the gland.

Measurements should be taken in the middle of the prostatic urethra, and at or as near as may be to the vesical orifice. The former are the more valuable. When the alteration in size or distensibility at the outlet is very marked the shape of the gland is usually too irregular for accuracy. The opening, for example, may be distorted into a semi-lunar shape by the growth of a median projection from behind, and give an antero-posterior diameter that is altogether deceptive. In the middle of the prostatic urethra this rarely happens. In this part an increase in the antero-posterior diameter, without any great change in the transverse one, always indicates overgrowth of the lateral lobes. If the measurement diminishes rapidly towards the bladder, and if the urethra is at the same time increased in length, not only are they enlarged, but they have raised up between them a fold of mucous membrane across the posterior boundary of the outlet. And if there is at the same time a distinct angle in the posterior wall, stopping the passage of an ordinary catheter, the fold is of considerable thickness, projecting forwards as well as upwards, and contains an outgrowth of glandular tissue. In other words, the vesical orifice is raised, displaced forwards, and surrounded either by a continuous collar or by projecting eminences which meet together over it when the bladder contracts. 
The lateral diameter deserves an equal amount of attention. Normally the transverse section of the prostatic urethra, about its centre, is crescentic in shape, with the convexity forwards. When there is general enlargement this is altered to a tri-radiate star. Increased width is a sure indication that there is overgrowth in the posterior wall, involving the urethra, if not the vesical outlet.

It is more difficult to ascertain the extent to which the urethra is displaced in the substance of an enlarged prostate. Usually there is very little increase in front, but I have met with one instance in which this was so great that the channel lay a long way behind the centre. In another in which prostatectomy was performed, it is recorded that the anterior
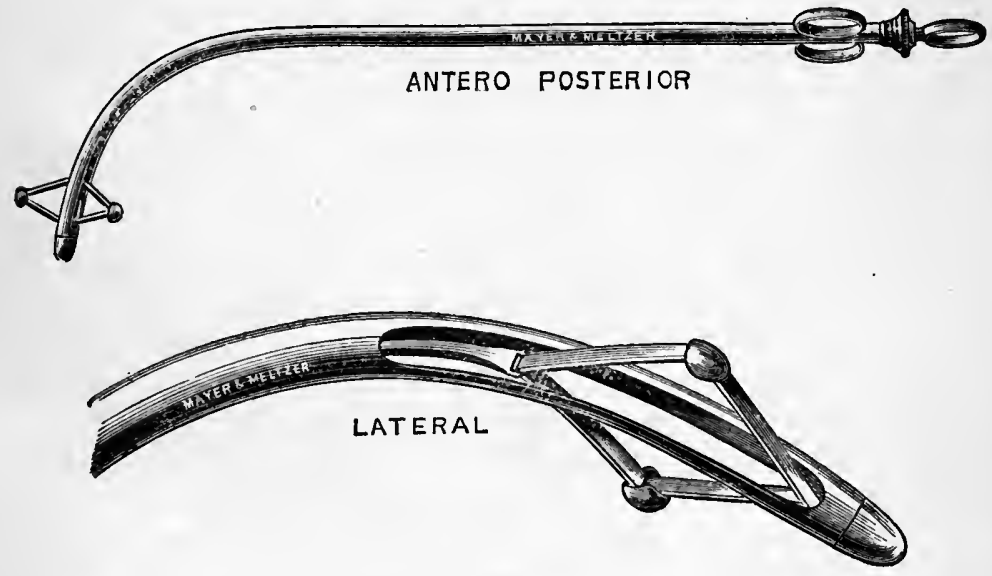

growth was the main and almost the sole obstruction (Wishard, "Journal of Cutaneous and Genito-Urinary Diseases," March, 1892). It may be suspected if, when the prostate is distinctly enlarged, the antero-posterior diameter of the urethra is not increased, and the canal is unusually straight.

Messrs. Mayer and Meltzer have made for me a modification of the ordinary form of urethrameter, which I have found exceedingly useful for this purpose. There are two forms of it. Each consists of a diamond shaped metal frame, hinged at the four angles to allow of expansion and contrac- 
tion, and enclosed in the curve of a prostatic catheter, part of the wall of which has been cut away. In the one the sides are open to allow of lateral expansion; in the other the front and back for antero-posterior measurement. The projecting angles in each case are protected with little rounded buttons, so that the mucous membrane may not be hurt or bruised. Expansion and contraction are effected by means of a stilet gliding backwards and forwards in the shaft and terminating in a finger ring. The measurement scale, which is marked upon the handle of the stilet, gives at a glance the width to which the frame is expanded at the moment. By means of these, introducing first one and then the other, the interior of the prostatic urethra can be mapped out with perfect accuracy, and in many instances, owing to the difference in the resistance presented by the walls, the density of the growth can be ascertained as well ("Lancet," December 17 , 1892).

\section{The State of the Bladder and Kidneys.}

Enlargement of the prostate derives all or almost all its importance from the influence it exerts upon the bladder and kidneys. The diagnosis; therefore, cannot be considered complete until an estimate has been formed of the effect it has produced upon the urinary organs. The amount of residual urine, the condition of the mucous membrane of the bladder, the tone of its muscular wall, and the secreting power of the kidneys must be ascertained before a definite line of treatment can be adopted.

The amount of residual urine is estimated by passing a catheter after the patient has emptied his bladder so far as he can. This must be done on several occasions, and under different circumstances, before forming a definite opinion. Patients rarely face an examination of the urethra unless the symptoms have been growing rapidly worse of late. And, partly because of this, partly from nervousness, it often happens that the amount appears to be larger than it really is. Not unfrequently the tone of the bladder improves and the quantity diminishes considerably in the course of a few days.

The precautions that must be taken will be detailed later. 
Estimating the amount of residual urine that is present in a case of enlargement of the prostate is often a very serious proceeding, owing to the risk of conveying septic organisms from the urethra into the bladder and setting up septic cystitis and pyelonephritis. It should never, therefore, be undertaken, even in a patient who has become habituated to the use of instruments, and has probably acquired a certain degree of immunity, without thorough preparation.

The tone of the muscular coat of the bladder and the strength with which it can act are best estimated by watching the changes in the amount of residual urine, and the force with which the stream is propelled through a full-sized catheter. When the tone is good, the urine is ejected vigorously without any conspicuous exertion on the part of the abdominal muscles, and the stream retains its strength to the end. When, on the other hand, the muscular coat is failing it flows away quietly, rising and falling with the respiratory movements, and if the patient is lying down and does not cough or make any particular effort the last few ounces of urine are only expelled when pressure is made with the hand over the pubes. Very often the stream stops long before the bladder is empty, and begins again when the catheter is pushed in a little farther or drawn a little back. The bladder no longer contracts uniformly from all sides on to the orifice, expelling its contents to the last drop. It collapses and falls into loose folds, which hang down over the opening, and leave a pouch filled with urine behind.

A definite opinion as to the extent to which the bladder has lost tone cannot be formed from a single examination. Over-distension, for example, makes it so much worse for a time that a wrong impression is easily formed. The first few ounces when a catheter is passed in such a case may be expelled with vigour, owing to the elastic recoil of the tensely stretched wall. As soon as this is spent the urine flows away as if the bladder were absolutely powerless. When the distension has been extreme there may be no evidence of muscular action for days. After a while, however, the bladder begins to recover, and though the tone is seldom quite so good as it was before, especially if there used to be much residual urine, a great deal of it may be recovered. 
Allowance, too, must be made if a catheter has been constantly employed for some time past. The persistent use of a catheter seriously weakens the power of the bladder. The muscular fibres, relieved of all work, undergo degeneration, and when called upon no longer possess the strength. It has been said that recovery of the power of voluntary evacuation is impossible after the habitual use of a catheter for two years: that after so long an interval the muscular coat can never regain what it has lost. It is probable, however, that the permanent character of the loss depends a great deal more upon whether there has been cystitis or not. If inflammation has never broken out or has never been sufficiently severe to affect the muscular layer, the power of contraction can be recovered after a much longer interval than this. I have known instances in which voluntary control has been regained after an interval of six and even seven years. There is a material difference between the loss of tone which results from disuse and that which is caused by inflammation. The bladder will recover from one if the patient's general health is good; the muscular fibres have not been destroyed. The other is undoubtedly sometimes permanent.

The capacity of the bladder again must be taken into consideration in estimating the tone retained by its muscular wall. It may be able to deal satisfactorily with a small quantity of urine and fail with a large one, the fibres becoming tired out before their work is finished. A bladder that can only hold a small amount of urine is able to contract efficiently with much less expenditure of strength than a large one. The length of time through which the muscular coat is able to maintain a state of active contraction, however slight, is probably the best test of its condition. I have tried by means of a manometer attached to the side of a catheter to obtain a more accurate record of this, but the result has not proved satisfactory. The novelty and the amount of apparatus prevented, it seemed to me, the patient from doing himself full justice.

The state of the mucous membrane of the bladder is not difficult to ascertain. In a certain proportion of cases it can be inspected directly, if it is wished, by means of the cystoscope. If this cannot be introduced, or if such an amount of instru- 
mentation is not thought advisable, an equally accurate opinion can be formed from the appearance and the character of the deposit left by the urine, the frequency of micturition, the severity of the pain that accompanies it and the other symptoms that are present.

The most exact information is obtained by examination of the urinary sediment. This may be collected either by allowing the urine to stand for some hours in a conical glass, or, preferably, especially if the quantity is small, by a centrifugal machine. The saving of time is considerable, and the urine is examined while it is still fresh, before any additional changes have made their appearance in it. As the number and variety of the micro-organisms present are of great importance, everything with which the urine can come into contact should be sterilised by heat or rendered aseptic in some other way; and if the urine to be examined is passed without the aid of a catheter, the urethra should be washed out first with boracic acid solution, and only that portion saved which is passed the last, so as to avoid as far as possible the fallacy of mistaking urethral organisms for vesical ones. In cases in which cystitis is present already there is but little fear of this. Some of the organisms which abound in the urine under those circumstances may have come down from the kidney into the bladder with the stream of urine, others may have been washed out from the urethra: but the number present is so enormous that the addition of a few more makes little or no difference.

The deposit should be examined under the microscope, and if there is any doubt as to the presence of micro-organisms, cultures in gelatine or on cooked potato or other media should be made from it. Pus corpuscles and bacilli are always present if the mucous membrane of the bladder is inflamed. As I have shown in my work on "Inflammation of the Bladder," there is no such thing as catarrhal cystitis. Inflammation of the bladder, with the possible exception of that which is caused by chemical irritants, such as cantharides, and that which is due to specific organisms, such as the tubercle bacillus, is always purulent, whenever it occurs, and is always caused by the presence of pyogenic organisms.

If the attack is slight and the mucous membrane not 
seriously affected, the amount of pus is small and the deposit consists mainly of the inorganic salts of the urine which have been precipitated, and detached epithelial cells, forming a light floating cloud. If the inflammation is more severe, the deposit soon sinks down and forms a dense greyish layer at the bottom of the vessel. Very few epithelial cells can be found in it, and those for the most part ill-formed. It consists almost entirely of pus corpuscles, with myriads of septic organisms, and a variable amount of blood (sometimes only a little, sometimes a great deal, but nearly always some) mixed with the ordinary deposits found in acid or neutral urine. The mucous membrane is swollen and intensely congested. The surface bleeds at the slightest touch. The epithelial cells that cover it are ill-developed and melt away before they are fully formed. They are no longer able to defend the softened and œdematous structures beneath; and the septic organisms easily penetrate through the protecting layers and spread in all directions in the interstices between the tissues. Often in these cases the urine has a faint and most unpleasant odour : sometimes it is plainly fæcal, due to the indol formed by the bacillus coli during its growth.

If the urine is ammoniacal, and if after standing an hour or two, a slimy tenacious mass of pus mixed with earthy and triple phosphates settles down to the bottom and clings to the side of the vessel when it is inverted, the condition of the mucous membrane is much more grave. The interior of the bladder is coated over with a deposit similar to that which collects after the urine is passed. Underneath it the surface is raw, ulcerated in places, so that it bleeds at the slightest touch, and is stained by hæmorrhages. All the coats are in a state of intense congestion; the fibrous tissue is swollen and softened; the muscular fibres are beginning to degenerate and break down; many of the vessels are blocked, and all the lymphatic spaces and the interstices between the tissues are filled with inflammatory exudation and masses of septic organisms.

The occurrence of cystitis marks a definite stage in the life history of enlargement of the prostate. The mucous membrane may be congested. There may be retention of urine and atony. The muscular coat may be seriously 
weakened from disease and chronic congestion. So long as septic inflammation does not occur, so long as pyogenic organisms never gain entrance, or gaining entrance, fail to make good their hold, the wall of the bladder, if the cause is removed and the patient not too infirm, is, in the vast majority of instances, capable of recovery. When cystitis sets in, unless it is checked at once, neither the bladder nor the kidneys can escape without permanent injury.

As Melchior of Copenhagen has shown ("Cystite et Infection Urinaire," Steinheil, Paris), the worst forms of septic cystitis, ending in septic pyelonephritis and acute septicæmia may occur with urine which is intensely acid throughout; the bacillus coli, which is nearly always the immediate cause of septic inflammation in the urinary tract, can grow with equal virulence in acid, neutral, or alkaline urine.* But there can be no doubt that when the organisms that cause ammoniacal decomposition of the urine gain entrance as well, the local destruction is much more rapid. The carbonate of ammonia that is formed is such an intense irritant that tissues which, uninjured, might have been able to withstand the assaults of the bacillus coli, are easily overcome by the two acting together; ulceration and sloughing of the mucous membrane are not uncommon and even gangrene may occur.

Other changes in the condition of the bladder can be inferred from the mode in which micturition is performed. Long-standing difficulty without much residual urine is suggestive of fasciculation of the wall. Sudden and unaccountable changes in the quality and quantity of the urine, and the slipping of a catheter into another collection of urine, after it has apparently emptied the bladder, usually indicate the presence of sacculi. These conditions, however, and the state of the orifices of the ureters can only be made out for certain by the cystoscope.

Increased frequency of micturition without pain is a sign of

* It is doubtful whether the bacillus coli possesses the power of decomposing urea or not. According to Melchior it does not possess it, at any rate in sufficient degree to render it important on that account. Certainly its power is not nearly so great as that of the proteus of Hauser and some other bacilli; but perhaps it varies in virulence according to the mode of culture. 
distention and residual urine. When pain is present with it the mucous membrane at the neck of the bladder is congested or inflamed, and the degree of urgency may be taken as the measure of the changes this has undergone. So long as it is not very great there is merely a certain amount of hyperæmia; the surface is not broken. Strangury, burning pain at the neck of the bladder, spreading down to the end of the penis, and accompanied by the spasmodic ejection at frequent intervals of a few drops of urine, never occurs without some intense source of irritation, such as calculus or inflammation. In the worst cases there is often a small ulcer or fissure between the folds of mucous membrane at the neck, causing pain and kept from healing by the spasm it excites, much in the same way as the same complaint in the neighbouring bowel.

The condition of the kidneys requires to be very carefully investigated in all cases of enlargement of the prostate. The signs of renal degeneration in its earlier stages are very vague and ill-defined. Failure in health and strength without any obvious cause, loss of energy, emaciation, and the sudden assumption of the aspect of old age are highly suggestive. The patient is always catching cold and is upset by apparently trivial causes. The hair turns grey rather suddenly, the skin grows harsh, the tongue becomes dry and coated, and the appetite capricious. Feverish attacks are common, and the patient complains that he is always suffering from vague pains across the loins, and that he is never really well. The urine is examined, and the amount passed in the twenty-four hours is found to be unduly large and the specific gravity unduly low. There is no albumen, however, and although fibrinous casts may be occasionally detected, they are by no means common.

The mere diagnosis, however, of renal degeneration is not sufficient. The cause must be found out as well. It may be a symptom of general arterial sclerosis, or it may be the consequence of backward urinary pressure, or as Albarran suggests ("Etude sur le rein des Urinaires," Steinheil, Paris), of the action of pyogenic organisms not sufficiently active or numerous to excite suppuration. It may be of constitutional or of local origin, or of both together. The effect, so far as 
the urine is concerned, is almost the same, although it follows more slowly in the one than in the other. In each case there is the same premature old age; but the one is dependent upon a cause which cannot be removed, however much its consequences may be averted, the other upon a cause which sometimes can.

When the two occur together, which is not unfrequent, the difficulty of assigning the proportionate share to each is, as might be expected, very much greater.

Careful comparison of cause and effect, especially of the relative time at which the symptoms have made their appearance, enables an opinion to be formed in most cases. In some the urinary obstruction is of recent date, the amount of residual urine is out of proportion to the size of the prostate, the general symptoms are well-marked, and there is evidence in other parts of the body of the vascular and other changes usually associated with arterial degeneration. In others the prostatic symptoms are distinct and old-standing, while the arteries that are accessible to observation show little or no evidence of disease. In the former case the renal degeneration may be of constitutional origin, in the latter it is much more likely to be local.

I have not been able to find any constant relation between the consistence of the enlarged gland, as felt through the rectum, and the presence of arterial degeneration. It has been said that in markedly atheromatous patients the prostate undergoes fibroid transformation at a comparatively early stage of enlargement, and that consequently it never attains any conspicuous dimensions, although this does not prevent it becoming a serious source of obstruction. Such a coincidence is, however, by no means invariable, and it must be regarded as a coincidence only. I have met with enormous adenomatous growths in patients whose arteries were distinctly atheromatous, and conversely with a gland of almost scirrhous hardness when the vessels were to all appearance perfectly sound.

The onset of septic nephritis is shown by the effect that it has upon the urine, and by the increased severity of the constitutional symptoms. The presence of casts of various kinds, the increase in the amount of albumen and the 
diminution in that of urea are the most significant of the changes in the former, independently of those that arise from the inflammation of the bladder. Rapid loss of flesh and strength, failure of mental energy, incipient delirium (especially at night), vomiting, anorexia, with a dry, red tongue, irregular temperature, and a quick, feeble pulse are the most characteristic of the latter. 


\section{CHAPTER VII.}

The General Treatment of Enlargement of the Prostate.

THE general treatment of enlargement of the prostate deserves more consideration than it usually receives. The occurrence of acute complications, such as retention of urine and cystitis, in a large proportion of cases can be traced back. directly to an attack of prostatic congestion arising from some act of carelessness.

Diet is of great importance. By the time the patient has reached the age at which prostatic obstruction usually causes symptoms he ought to know what agrees with him, and should confine himself to that. But most people are so constituted that definite rules are necessary.

Meals should be light and frequent. Long and late dinners with many courses are most injurious. The plainer and simpler the diet the better. Rich food, highly-dressed dishes with elaborate sauces, and a great variety of food at the same meal should be strictly avoided. Most attacks of prostatic retention originate in what is popularly called congestion of the liver.

Meat and highly nitrogenous foods, such as cheese and eggs, should be partaken of very sparingly. Most people consume daily a great deal more than they require. So long as the patient is leading an active, vigorous life the excess may be disposed of without leaving any apparent effect, but as a rule the amount of exercise taken each day, and the energy with which it is taken, begin to diminish about the time when prostatic symptoms grow troublesome. The requirements grow less, and the ability to dispose of any surplus without inconvenience disappears.

This caution is especially needed when the urine is highly acid, and inclined to deposit-crystals on standing. Owing to 
the congestion at the neck of the bladder the mucous membrane is intensely sensitive. The least excess of acid in the urine irritates it at once, and by causing increased frequency of micturition makes the congestion worse. Fish, poultry, and gaine do not appear to have the same tendency, and should be used as far as possible as substitutes.

Ripe fruits and vegetables, except such as tomatoes, rhubarb, and asparagus, which have a very definite effect upon the urine, may be taken in reasonable amount. They tend to keep the action of the bowels regular, and diminish the acidity of the urine. Care, however, must be taken that this is not carried too far, and that it is not rendered alkaline. Highly-spiced or salted articles of diet, pastry, and sweet dishes generally must be avoided.

The quantity of fluid taken daily is to a great extent a matter of personal habit acquired during previous life. Many patients, especially when the kidneys are beginning to fail, require and are better for a large amount. Others can do with very little. Quantity has some influence upon the frequency of micturition, but this depends much more upon the amount of residual urine, and the condition of the mucous membrane at the neck of the bladder. A glass of hot water, taken the last thing at bedtime, sometimes diminishes the number of micturitions in the night by making the urine less concentrated and less irritating. On the other hand, when the urine is already very dilute, it may make matters worse.

Alcohol in small quantities, and taken at meal time, is generally beneficial to those who have been accustomed to its use. It enables them to make the best use of their food with the least expenditure of energy. It should never be taken except with food, and should not be taken the last thing at night. The particular form must depend upon the individual, but, speaking generally, the more diluted it is the better. Champagne, sherry, and beer are perhaps the most generally injurious. Whisky and gin enjoy the highest repute, but they must be old. There can be no question that newly-fermented wines and spirits are not good.

Milk may be taken freely by those who can digest it well. If the quantity at first is small and it is increased slowly, exceptions are not often met witl. Coffee is irritating to 
many people. The question of tea depends upon the length of time it is allowed to stand and the amount of tannin it contains. Hard water should be avoided. The salts of lime which it contains are partly excreted as oxalates in the urine; and crystals of oxalate of lime are especially irritating.

The bowels should never be allowed to become constipated. Straining has almost as great an influence upon the prostatic plexus as it has upon the hæmorrhoidal. Great care must be taken in the choice of laxatives, avoiding those which are liable to increase the congestion of the rectum, changing them frequently and combining them with tonics. Active purgation is as injurious as constipation. In the later stages; when the bowel has lost its tone, and the control over the sphincter is failing, there is always a tendency to hæmorrhoids and prolapse. If this occurs the irritation spreads to the neck of the bladder, the frequency of micturition is greatly increased, and the patient's condition may become. one of extreme misery.

Clothing must be warm but light, flannel, silk, or merinobeing worn next the skin. At the same time excess must be carefully avoided. Overclothing is scarcely less injurious than the reverse. Patients with enlargement of the prostate are peculiarly susceptible to chills if they are tired or overheated, and the least chill may cause retention of urine. The loins especially must be well protected, and the lower extremities kept warm, and, above all, dry.

Hip baths as hot as can be borne, just before getting into bed, are sometimes of service in relieving the irritability at the neck of the bladder; but immersion should not be prolonged for more than a minute or two.' In the morning the temperature of the bath should be guided by the energy with which the circulation reacts. Light general friction after: wards is very beneficial from the influence which it has upon the blood supply of the skin.

Exercise in moderation is essential, but it must be of a kind that is suited to the condition of the patient. It helps to maintain the general health; prevents attacks of local: congestion, and keeps the liver and the bowels in good order. The age and the physical condition of the patient must; of course, be carefully considered, and exercisc must always: 
stop short of fatigue. Riding, long railway journeys, and anything that tends to increase the congestion of the pelvic organs must be prohibited. When for any reason exercise cannot be taken, general massage is an excellent substitute, maintaining a high standard of activity for both the circulalatory and excretory organs.

No drugs have the least influence upon the rate of growth of the gland. Some, however, are of service in helping to maintain the tone of the bladder and in controlling the reaction and the concentration of the urine.

Ergot and nux vomica have the highest reputation. Of the latter there can be no doubt. When the tone of the bladder is failing it is of great value, whether given by the mouth, or as strychnia, hypodermically. Ergot, on the other hand, is probably useless. It was given originally because of its action on the gravid uterus and the homology that was supposed to exist between that organ and the prostate; but it has never been proved to have the slightest influence.

Belladonna should never be given in cases in which the tone of the bladder shows the least sign of failing; and the same statement must apply to hyoscyamus and conium, although the effect that they produce is very much less important.

A large number of drugs on the other hand have marked influence upon the urine, and so indirectly upon the urgency and persistence of the symptoms.

Excess of acidity leading to the deposit of uric acid crystals or of acid urates, and causing increased frequency of micturition, can be met in this way. In many instances it is dependent, more or less immediately, upon the condition of the liver, and can be rectified at once by small doses of blue pill or calomel. Simple dilution, increasing the quantity of fluid taken during the twenty-four hours, is occasionally sufficient. It has been shown experimentally to have a distinct effect upon the discharge of nitrogenous waste. If this fails the acidity can be controlled by the administration of alkalies, citrate of potash, or lithia, for example; or the liquor potassæ or the bicarbonate of soda well diluted. Care, however, must be taken not to carry the change in reaction 
too far; alkalies should never be given as a routine treatment in irritability of the bladder.

Generally speaking it is advisable when the urine continues to be unduly acid to stop the consumption of sugar for a time, and, if the patient feels the deprivation, to substitute saccharin. Piperazine, in five-grain doses, three times a day, I have found of considerable value, especially when there are numerous uric acid crystals. Some small part of the benefit may be due to its influence as a diuretic, for the amount of urine passed when fifteen grains a day are taken for any length of time is considerably increased.

Alkalinity of the urine may be dependent upon decomposition of the urea in the bladder, and later in the pelvis of the kidney; or upon excess of fixed alkali at the time of secretion. In either case it should be checked as soon as possible. It leads to the precipitation of the phosphate of lime, and when the urea decomposes, of the ammonio-magnesian phosphate as well, so that calculi and incrustations form upon the wall of the bladder and in the pelvis of the kidney.

Very little can be done for urine that is alkaline at the time it is secreted. In many instances, unless the reaction is merely of a temporary character, as, for example, during the period of active digestion, it is evidence of some grave disorder of assimilation or metabolism, and the treatment of the urinary affection must be subordinated to that of the general symptoms. The drugs that are of most avail for increasing the acidity of the urine are the acid phosphate of soda, boric acid, which may be given in seven or ten grain doses three times a day, and benzoic acid or, because it is more soluble, benzoate of ammonia. If the two latter, as sometimes happens, disagree with the patient and upset digestion they may be given in ten grain doses in capsules which will pass unaltered through the stomach into the intestines, or as Belfield recommends with borax and boric acid, three grains of each, four to six times a day. Mineral acids are of no use except in so far as they act as tonics and improve the general health; and the organic acids for the most part make matters worse.

Alkalinity arising from ammoniacal decomposition, on the other hand, is of local origin, and is to be met by local 
measures mainly. A certain amount of benefit, however, is derived from drugs that increase the acidity of the urine, or act as antiseptics. Urotropin, combined with the acid phosphate of soda is the most useful. Boric acid, seven to ten grains, three times a day, sanmetto and salol, in cachets containing three grains, one with each meal, are also of service. Even when the cause is a permanent one they tend to check decomposition, and as soon as this is under control, the alkalinity disappears. Quinine, independently of its general therapeutic value, has considerable influence on urinary fermentation, probably owing to its bactericidal powers; and though they cannot replace urotropin and salol, salicylic acid, salicylate of soda and naphthalin are sometimes of use in the same way.

Congestion of the mucous membrane lining the urinary passages can be checked by small doses of turpentine and other resins given in capsules. Sandal wood and eucalyptus oil are the least objectionable, but copaiba with cubebs, sometimes answers better than either of them. Probably the benefit that has been observed to follow the administration of buchu is due to the volatile oil it contains. Belfield speaks favourably of an emulsion consisting of small doses of turpentine, powdered cubebs, and belladonna, and of pichi (Extr. Fabianæ liquid, $m x$. to $m \times x x$.).

Of the large number of other drugs that are frequently given in this condition, such as pareira, uva ursi, triticum repens, \&c., it is very difficult to say anything definite. Undoubtedly a certain amount of benefit is often experienced while they are being taken, but it is very difficult to say whether it is due to any action that they possess, or to other remedies taken with them, to local treatment, to better observance of ordinary dietetic rules, or to mere dilution. On the whole the positive evidence in their favour is very small. 


\section{CHAPTER VIII.}

The Local Treatment of Enlargement of the Prostate.

Palliative Measures: the Maintenance of the Urethra-The Prevention of Retention and of Residual Urine-The Prevention of Irritability of the Bladder and Cystitis.

EnLaRgement of the prostate does not, of itself, require any local treatment. Unless it obstructs the exit from the bladder or the rectum, or causes vesical irritation, it is not dangerous to life, and in the majority of cases its existence is not even suspected. The treatment, when it does give rise to these consequences, consists either in removing the offending outgrowth, or in leaving it alone, adopting palliative measures, and meeting the various complications as they arise.

\section{Palliative Measures.}

The aim in view is to maintain a clear and open route through the urethra; to avoid the collection of residual urine and the occurrence of retention; and to prevent irritability of the bladder and cystitis.

If this can be accomplished more energetic measures are not needed.

\section{The Maintenance of the Urethra.}

In the early stages of the disease a certain amount of benefit may be obtained by mechanical dilatation, passing full-sized, highly polished steel sounds, and leaving them in situ for a quarter of an hour or twenty minutes. They must be as large as the membranous portion of the urethra will admit, and must, of course, be used with the greatest gentleness. 
They have no influence upon the rate of growth. This goes on as fast or as slowly as it did before. All they do is to compress the mucous membrane, empty the blood-vessels, and prevent the walls of the urethra being continually squeezed together. I have found them of service when the lateral lobes are enlarged and the urethra flattened, and when there is risk of retention owing to insufficient muscular hypertrophy. The stream in these cases is never good, though the amount of residual urine is often small. In many such, radical treatment is either inadmissible or cannot be strongly urged. If the wall of the bladder can be saved, before it has been overstrained, by rendering the outlet a little more easy, atony may be prevented, or at least postponed.

It is only when the rate of growth is very slow that sounds can be expected to do any good. And even then it must be insisted that their effect is only beneficial for a time. As the patient grows older the obstruction becomes greater and the muscular power less; and at length, even if cystitis is warded off, sounds have to be replaced by catheters with all the risk of infection in an enfeebled bladder. They are only palliative at the best, and that in a few cases, and only so long as the changes are slight.

Sounds that are used for this purpose are longer than ordinary catheters and are made with a moderate prostatic curve. The point and the shaft are equal in size to a No. 8 English. The enlargement begins a little distance from the lip, reaches it full size before the middle of the curve, continues the same for an inch or an inch and a half and then gradually diminishes to the junction of the curve with the shaft. Many patients after a certain amount of practice are able to introduce an instrument equal to No. I6 English, but it is not advisable to begin with one larger than No. 12. There should be no hesitation in enlarging the meatus if it is found necessary. The sounds are passed once or twice a week, the last thing at night, preferably when the patient is lying down, and are retained for from fifteen minutes to half an hour.

By using these I have been enabled in some cases to postpone more active treatment for several years. Much depends 
upon the care which the patient takes in carrying out instructions. With sounds, especially when they are highly polished, there is much less risk than with catheters; but with both alike there is danger that organisms may be carried into the bladder and set up suppurative inflammation. On the whole, it is better that the sound should be passed before emptying the bladder. It is true that if the bladder is emptied first, the urine passing down the urethra cleanses it to a certain extent, and washes out some of the organisms that adhere to the walls; but very imperfectly. The last drops of urine that come away generally contain as many organisms as the first. Sometimes they contain an even greater number, probably because, passing very slowly down, as they are apt to do in cases of enlargement of the prostate, they remain longer in contact with the urethral surface. It is better therefore that the sound should be introduced before micturition. Germs are very likely to be carried in, whichever plan is adopted, but if the bladder is emptied at once, and thoroughly, after the sound is withdrawn, it stands a better change of being free from them whatever may be the state of the urethra, than if it is emptied first.

This method of maintaining the patency of the urethra is very different from that of over-stretching the canal, which has been proposed for cases of lateral hypertrophy in which the passage is narrowed or tortuous. Over-stretching is to be done under an anæsthetic, and the dilatation is to be carried to the farthest degree consistent with safety. It has been advised on the grounds that dilatation with sounds is certainly useful; that the prostate has sometimes been found to be atrophied in cases of stricture; and that previous attempts (which certainly were not very successful) were made at a time when asepsis in genito-urinary surgery was unknown. The two methods, however, are based upon entirely different principles; and putting aside other difficulties and taking no note of the risk always attendant on forcible dilatation, stretching of the urethra cannot be recommended. No reliance is to be placed upon the comparison with the atrophy of the prostate that is sometimes found in cases of stricture. Atrophy under these conditions is very far from common, and even if it were common, there is no 
proof that it is due to the mechanical pressure of the urine. There are other consequences of stricture, chronic epididymitis, for example, due to the passage of catheters or the use of injections, which may not unreasonably be held to have quite as great an influence in producing this result.

\section{The Prevention of Retention.}

Inability to empty the bladder may arise from failure of the expulsive power, or increase in the resistance, or from both together. The object of palliative treatment is to restore the normal balance without operation.

A. The treatment of the loss of power.

Diminution in the strength of the muscular coat of the bladder arises from various causes.

It may be induced by one single act of extreme distension -atony in the strict sense of the term. This may occur even in young men from prolonged voluntary retention of urine. In older men, suffering from enlargement of the prostate, it is far more common, but then there are usually other causes at work as well. Generally speaking, if the urine is drawn off at regular intervals, and the bladder is allowed time to recover itself, loss of power caused in this way slowly passes off. But it may be permanent, even in young men, and in old ones it almost always leaves behind it some degree of permanent disability.

In other cases loss of power is caused by a valvular outgrowth at the neck of the bladder, or lower down, in the floor of the prostatic urethra. The more vigorous the attempt at evacuation, the more tightly the orifice is closed. The resistance in front grows greater with each effort; and the muscular coat, in the face of such an obstacle, soon ceases from efforts that must be futile, and yields and gives way.

Disuse is another cause, the muscular fibres undergoing degeneration. This is one of the necessary consequences of the habitual employment of catheters. The muscular coat of the bladder, never doing any work, gradually loses the power of working. The prognosis, if there is no other reason for the loss of power, is good; the bladder may recover even after many years of habitual catheterism. 
The chronic congestion that is so common in cases of enlargement of the prostate is another cause. It is imma. terial whether the congestion is the result of atheroma, or the effect of persistent straining upon a valveless prostatic plexus. The muscular fibres are badly nourished; the blood no longer circulates freely around them; all the interstices between them are filled with stagnant lymph, and their power of working is enfeebled at the very time that the call upon them, owing to the swelling at the neck of the bladder, is increased.

Chronic inflammation is still more injurious. At first it affects only the mucous membrane lining the bladder; but, by degrees, the septic organisms penetrate deeper and deeper into the muscular layers, injuring and weakening all they come near. The walls of the bladder may be thickened and fasciculated, but the increased thickness is rarely due to muscular hypertrophy. It is far more common to find the muscular fibres wasted and replaced by lowly organised fibrous tissue, so that the contractile power is diminished, though the rigidity may be increased.

Treatment is rendered still more difficult by the fact that nearly always there are several of these causes at work together.

Over-distension or anything that might throw the least extra strain upon the bladder must be carefully avoided. If the loss of power is complete a catheter must be passed at regular intervals; if it is only partial, if, that is to say, there is a certain amount of residual urine, it must be passed two or more times a day, according to the quantity. The patient may be encouraged to expel the urine through the instrument as forcibly as he can, with the view of exercising the muscles of the bladder against a resistance they can easily overcome; but much must not be expected from this. It is nearly always the abdominal muscles that are employed.

Strychnia, as already mentioned, is of undoubted service. It may be given by the mouth with iron, the mineral acids, and other tonics, or hypodermically. Hydrastis canadensis is highly spoken of, and also ergot. It is doubtful if injections of ergotin and sclerotic acid are of the least use. I have seen then tried on several occasions, but never with any benefit. 
Cold douching over the abdomen and loins, night and morning, and massage applied to the lumbar region, are beneficial at times. The effect is probably due to the increased activity of the circulation through the nerve centres, or to the improvement in general muscular vigour, rather than to any influence upon the bladder itself. The bladder may be washed out with cold water through a catheter, but the effect is not sufficiently sudden to excite muscular contraction. If it is tried great care must be taken not to distend the cavity. Cold applications to the rectum are only of use when there is active congestion or inflammation of the gland.

I had hoped much from the application of galvanism in these cases of loss of muscular power, but although $I$ have tried it in a fair number, I have not met with a great degree of encouragement. A slowly interrupted current is the most suitable, reversing the direction from time to time. One pole is placed over the lumbar region; the other over the pubes, in the rectum, or by means of a properly contrived bougie-electrode, in the bladder. The urine is drawn off first and a sufficient quantity of boiled salt and water injected to unfold the walls without distending them. Or a full-sized soft red rubber catheter may be passed into the bladder; a funnel attached to the end; and bladder, catheter and funnel filled with salt solution. The circuit can then be closed by dipping one of the electrodes into the fluid in the funnel. The recovery of power if the atony has lasted any length of time is always very slow; and the application should be continued twice a week for several months before coming to an adverse decision. I have never found galvanism give rise to any irritation of the mucous membrane.

$B$. The treatment of the obstruction.

When the bladder cannot be emptied voluntarily, because of the obstruction caused by the prostate, and radical measures are inadmissible or are declined, the symptoms must be relieved and the urine drawn off by means of catheters.

The kind of instrument depends upon whether it is used by a surgeon for purposes of diagnosis or to overcome some difficulty or by the patient as a matter of routine. In the 
former case a metal instrument is to be preferred, in the latter the softest that can be introduced. Whatever material they are made of, the eye should be perfectly smooth and rounded; the end beyond the eye should be solid, so that there is no receptacle to collect dirt, and the interior should be as smooth and polished as the exterior. If it is necessary in the one case in order that there may be the least possible amount of friction, it is equally necessary in the other that the urine may not soak into the fabric of the instrument or cling to the irregularities on its surface.

Red rubber ones are the softest and the most easy to keep aseptic. In many cases, however, they will not pass; either there is an elevation on the floor which they are unable to surmount, or the sides of the urethra are so closely pressed together that the catheter cannot separate them.

Black ones stiffened at the end and bent upwards at the point (coudé or bi-coudé) are often useful. If they fail, an English gum elastic which has been kept for some time overcurved upon a stilet will generally succeed; or Hey's well known plan may be tried of passing the catheter down to the obstruction, withdrawing the stilet, and at the same time, gently pressing the instrument onwards.

Olivary French catheters are of service when the canal is tortuous. The bulb insinuates itself more easily than the rounded end of the ordinary instrument. But the best and the most generally useful are the aseptic catheters made at my suggestion by Messrs. Maw, Son and Thompson. They are made in both shapes, coudé and olivary; the interior is as polished as the exterior, and they will stand boiling for five minutes every day for months together (provided they are kept straight while in the boiling water and are drained well afterwards), or continued immersion for weeks in boracic acid without being affected.

If there is very great resistance or if there are many false passages, metal instruments of the ordinary prostatic shape may be necessary. In any case the size should be as large as the membranous portion of the urethra will admit without inconvenience.

The introduction of a catheter for the first time is always a serious matter, whether the object is to find out the amount 
of residual urine that is present, or to give relief in a case of retention.

Whenever it is possible the patient should be carefully prepared. An aperient, preferably containing calomel, should be given the night before; the urine should be rendered aseptic by means of urotropin or salol and boric acid; and, especially in the case of those who have suffered from ague or who have lived in the tropics, full doses of quinine with a small quantity of opium should be given the day before and at the time itself. When there is the option the best time for passing an instrument is in the morning, while the patient is still warm in bed, an hour or two before he need get up. If this is not practicable the greatest care must be taken that there is no exposure to cold afterwards. The patient should be lying down, well covered up, but with the umbilicus exposed and the thighs slightly flexed and abducted. If he is standing it is more convenient for the operator, but there is a much greater tendency to syncope.

When residual urine is habitually present in the bladder the introduction of a catheter for the first time is attended by special risk and requires the greatest care. The hands, the skin of the penis and the prepuce must be thoroughly washed with soap and water first. Then the glans and the meatus must be cleansed and sterilised by sponging with a solution of corrosive sublimate, one part in five thousand, or of nitrate of silver; and the mucous membrane of the urethra disinfected, so far as is possible, by washing out the canal with a solution of boric acid. A Whitehead's or a Durham's syringe attached to an irrigator is the most convenient apparatus. The fossa navicularis is washed out first, and then the deeper part of the urethra, bit by bit, pushing the nozzle of the syringe up to the bulbous portion, and allowing the return current to stream out freely. The posterior portion of the urethra, like the bladder, is aseptic, so long as it is healthy.

This method of washing out the urethra is simple and is fairly effectual. It should never be omitted when a catheter is being passed for the first time. But it cannot be expected that patients will carry it out for themselves whenever they have to pass a catheter (I am usually satisfied if it is done 
once a day), and it must be admitted that it falls far short of scientific accuracy. It is, indeed, open to question whether complete disinfection of the urethra is within the range of possibility. The front part is full of germs even when it is perfectly healthy, and it is scarcely possible either to remove them or destroy them without using substances which act injuriously upon the mucous membrane. Petit and Wassermann, using large quantities of a four per cent. solution of boracic acid, entirely failed. Barlow, it is true, succeeded in a few instances by means of a solution of nitrate of silver (one part in a thousand) but he too met with many failures. Some of these germs are certain to be carried in by the catheter, but fortunately, although the bacillus coli has been found in the urethra, in the majority of instances the organisms that inhabit it (so long as it is not inflamed) are nonpathogenic.

If the urethra has been irritated already and is full of pyogenic organisms Melchior's instrument should be used. This consists of two tubes, one gliding inside the other. The outer is of metal and is sufficiently long to reach the deep part of the urethra. Its vesical end is closed with a film of rubber, held in place by means of a metal cap. The inner one is flexible, about the size of a No. 6 English (I always use one of my own aseptic instruments). As soon as the outer tube is in position the inner is passed down inside it, and pushed through the rubber film into the vesical urine. In this way the catheter that enters the bladder never comes into contact with the meatus or the mucous membrane of the anterior part of the urethra, and the risk of introducing micro-organisms into the bladder is reduced to a minimum. It is advisable to have the outer tube made with different curves, so as to suit the shape of the prostatic urethra in different cases.

It is essential that all catheters should be thoroughly cleansed as soon as they are withdrawn and sterilised before they are introduced. Metal and rubber instruments present little or no difficulty. They can be steamed, or immersed in carbolic acid or other disinfectants of sufficient strength for a sufficient length of time to destroy any germs that adhere to them. With my aseptic catheters disinfection is almost as 
easy. Ordinary gum elastic or black catheters, on the other hand, with a rough interior such as patients usually obtain for themselves, it is impossible to render aseptic. Disinfectants which are sufficiently strong destroy the surface and render them useless in a very short time.

Sterilisation cannot be carried out if a lubricant containing any kind of grease, such as oil or vaseline is used. No antiseptic of reasonable strength can penetrate through this, and soda is quite inadmissible in the case of most of these instruments. Even if the catheter is thoroughly washed with hot soap and water, some of the grease is certain to have penetrated into the interior through the eye and to escape. The lubricant should always be a soluble one. For some time past I have employed either boroglyceride or lanolin which has been sterilised and which is kept aseptic by the addition of a small quantity of carbolic acid. I prefer these to pure sterilised glycerine and to Guyon's preparations, which consist of equal parts of soap, water, and glycerine, with carbolic acid, $\beta$-naphthol or resorcin. The quantity prepared at any one time should be small, and it should be kept in a long, narrow, well-stoppered bottle.

The principles upon which sterilisation should be carried out are easily laid down. The details must vary with each case. And it must not be forgotten that the actual work must be entrusted in many instances either to nurses, or to the patients themselves, who are old men who often do not understand the importance of what they are wished to do, and still more often cannot wait to do it.

Each patient must provide himself with a number of catheters at least equal to the number of times the urine has to be drawn off in the course of the twenty-four hours. These should be kept in two of Nicoll's glass catheter cases, with rubber corks and metal caps, suspended vertically in their wardrobe or wherever it may be convenient. One of these cases is filled with the boracic acid lotion which should be renewed each day, or at least every other day. The other is empty except for a plug of absorbent cotton wool sprinkled with a few drops of formalin at the bottom. Side by side with these is an ordinary glass irrigator, filled with a strong solution of extract of soap, and fitted with an india-rubber 
tube and stopcock. Each catheter as soon as possible after it has been used is thoroughly washed out with the extract of soap, rinsed inside and outside with hot water, and dropped vertically into the tube filled with boracic acid. Once a day, or more frequently if necessary, all the catheters are taken out, boiled for five minutes, and placed to drain in the other Nicoll's case, where they remain in an atmosphere of formalin vapour until required.

The aseptic catheters made for me by Messrs. Maw, Son $\&$ Thompson stand this treatment very well. They are not affected in the least by boiling, if they are kept straight. The sterilisation is sufficient for all practical purposes; and after sterilisation they are kept free from risk of contamination until required. Moreover, the plan is so simple that it can be carried out by any nurse or patient; and if a catheter is required in the middle of the night at a moment's notice, when waiting even for an instant is impossible, it is ready to hand, sterilised and only needing the lubricant. I have tried many other methods, but I have not found one that is at the same time so simple and so efficient.

For travelling, catheters are carried most conveniently coiled up in flat circular boxes with a drop or two of formalin sprinkled in the interior to maintain a certain degree of asepticity. Leaden tubes with screw caps are useful for holding the boroglyceride or lanolin.

The object of these precautions is to prevent the occurrence of what is commonly called urinary fever, a form of fever which is liable to follow the passage of a catheter, and which, especially when there is any residual urine, may give rise to very serious consequences.

Urinary fever assumes a great variety of forms. Its immediate cause, however, is always the entrance into the circulation either of the products of septic organisms growing in some part of the urinary tract, or of the organisms themselves.

The simplest kind of urinary fever is a rigor or shivering fit. It begins, as a rule, not at the time the catheter is passed, but some hours later, after the first act of micturition. All of a sudden the patient is seized with a feeling of prostration and of intense cold, so that the teeth cannot be pre- 
vented from chattering. The skin becomes rough and livid. The face is pinched. The eyes are sunken and surrounded by dusky rings. The respiration is hurried and shallow, and the pulse small and frequent. From the first moment, even when the teeth are chattering and the patient shivering, the temperature begins to rise, and it continues through the period of dry burning heat that follows until sometimes it reaches $106^{\circ} \mathrm{F}$. As soon as it ceases profuse perspiration sets in, the face becomes flushed, the involuntary muscular fibre in the skin relaxes, and there is a sense of profound relief, although the weakness and exhaustion afterwards are often extreme.

In the majority of cases when once the immediate effects of the rigor have passed away there is no further trouble. The shivering does not return and the temperature remains normal. But every now and then cases occur in which the shivering fit is nothing less than the initial symptom of an attack of septicæmia. The rigors occur again and again. Now they no longer bear any relation to the time of micturition. Sometimes they recur every day, or every second day, with a persistence and a regularity that suggest some form of intermittent fever; more frequently they are quite irregular. No secondary deposits make their appearance. There is no cough or evidence of pulmonary affection. But the patient rapidly grows weaker and thinner; the strength fails more and more; there is perhaps an attack of diarrhœa, with peculiarly offensive motions; and the exhaustion advances until at length it passes into a condition resembling coma.

In other cases, again, those especially in which there is a large amount of residual urine with dilatation of the pelvis and atrophy of the glandular portion of the kidney, urinary fever assumes an entirely different form. For several days there is no rigor, or other symptoms of any note. Everything appears to be going on favourably. Then the bladder becomes irritable and the urine is found to be a little turbid, so that after standing for some time a grey deposit of pus collects at the bottom of the vessel. The amount that is passed may be increased or diminished, the difference depending in all probability upon the degree of congestion of the renal capillaries, and the reaction may remain acid or 
not; but nearly always the specific gravity falls, and a certain amount of albumen, more than can be accounted for by the pus that is present, makes it appearance. There is no rigor, though there may be several slight chills; the temperature rises one or two degrees and keeps up; the pulse increases in frequency and diminishes in strength; the tongue becomes dry and brown, especially down the centre; the appetite is lost; there is a tendency to nausea; the mind becomes a little unsettled, and then low muttering delirium begins at night and the patient sinks into what is called a typhoid state.

These are the most frequent forms of urinary fever met with after passing catheters. There is no doubt that they are caused by the catheters, directly or indirectly, for without them, or other instrument used in a similar way, they rarely occur. But until quite recently, when owing to the work of Rovsing and Melchior in Copenhagen, and of Albarran and Hallé in Paris, a flood of light has been thrown upon many obscure points in urinary pathology, no adequate explanation has been suggested for them. Now it is clear that, although allowance must be made for accessory causes such as renal degeneration or congestion due to shock and vasomotor paralysis, the immediate cause is the introduction of septic organisms into the bladder, and the entrance into the circulation either of these organisms, or of the toxins that they produce.

The rigors, for example, that occur after the passage of a catheter are caused by the absorption of toxins from the urethra. Septic organisms, as Melchior ("Cystite et Infection Urinaire," Steinheil, Paris) has shown, unless special precautions are taken, are swept into the bladder every time an instrument is passed. The toxins produced by these organisms are intensely virulent, sometimes perhaps more so than at others. The minutest quantity from a culture in broth or urine injected into the auricular vein of a rabbit is sufficient to cause collapse, diarrhœa and even convulsions. If there is the least abrasion of the mucous surface, these toxins are forced into the loose submucous layer by the increase in the pressure when the urine is driven down the urethra and symptoms of poisoning follow at once. 
In this way, and this way only, is it possible to explain and account for many of the clinical phenomena that are met with in urinary surgery. Rigors, for instance, seldom occur after internal urethrotomy if care is taken to render the urine and urethra thoroughly aseptic. They are more rare after operations upon old and dense cartilaginous strictures than after those upon recent ones, not because the germs are fewer, but because in the former absorption is not so easy: They occur as a rule,* not at the time of operation, or when a catheter is passed, but afterwards, at the first micturition, because the passage of the urine down the urethra raises the pressure and drives the toxins into the circulation. They seldom occur after external urethrotomy, because owing to the open wound there is no pressure. After lateral lithotomy they are very rare, even if the urine is septic, because the exit is so free. And for the same reason it sometimes happens that while every attempt at dillating a stricture causes a rigor, a free urethrotomy or rapid and complete dilatation cures the patient at once without the least reaction.

There are of course predisposing causes. In a case of internal urethrotomy recently under my care there was no rigor until the third micturition after the operation. The patient had become careless and stood out of bed with bare feet upon some oil cloth. Probably the amount of toxin absorbed in his case was not sufficient to cause a rigor of itself, and a chill was needed to start it. In the same way it is reasonable to account for the well known fact that urethral

* There are exceptions. A short time since I had occasion to operate upon a case of pyonephrosis, the pus in which was singularly offensive. It contained large quantities of indol and had a powerful freal odour, so that although no cultures were made, I have no doubt the bacillus coli was present in abundance. The patient was seized with a rigor before she had come round from the anrsthetic, while indeed she was being lifted from the operating table to her bed. Fortunately no ill results followed; the rigor lasted some ten minutes, was attended by a rise in temperature and followed by profuse perspiration in the ordinary way. I have no doubt it was caused by the absorption of some of the toxins during the operation. Probably they were driven into the recently incised tissues by my finger while I was exploring the interior of the sac to ascertain whether there was a calculus. Unhappily the result is not always so good. I have known death from acute septicamia follow exploration of a pyonephrosis within thirty-six hours. 
fever is especially liable to occur after the passage of a catheter in people who have once had an attack of malaria: the malarial poison prepares the ground and a much smaller dose of toxin is required. But in either case the immediate cause that determines the shivering is the same.

In the same way urinary septicæmia, which is a much more serious affection, is due to the entrance into the circulation not of the toxins produced by the organisms, but of the organisms themselves. The microbes have been found circulating in the blood during life and have been recognised in the spleen and other organs after death; and cultures and a series of inoculation experiments have been made from them by Melchior and others. Unlike ordinary urethral fever urinary septicæmia rarely begins at once. Nearly always there is an incubation period, sometimes of hours, sometimes of several days. From the first it is a progressive disorder, only too often advancing steadily from bad to worse in spite of all that is done for it. Fortunately it is rarely caused by the mere introduction of a catheter. Theoretically the smallest abrasion is sufficient, but as a matter of fact I have seldom known it occur unless there was some wound of definite size, such for example as that left by a division of a stricture or the removal of a tumour from the neck of the bladder.

And the same thing is true of the last and most insidious form of urinary fever, that which occurs in cases of residual urine dependent upon enlargement of the prostate. Like the urethral rigors and urinary septicæmia already described it is the direct product of the septic organisms that have been carried into the bladder. It begins invariably with septic cystitis and increase in the frequency of micturition. The urine, which may have been perfectly clear at the time the catheter was passed, two or three days later becomes cloudy and turbid. The reaction may remain acid, or it may become neutral; the quantity may be the same as before, or it may be increased or diminished; but the specific gravity nearly always falls, and if a specimen is allowed to stand or is placed in a centrifugal machine a grey deposit of pus and septic organisms always forms at the bottom. There may be but slight complaint of pain (probably because the post- 
prostatic pouch and not the neck of the bladder is involved); and there is but little fever; but from the first the tongue becomes dry and red; the pulse is quick and feeble, and there is wandering at night. Then the septic inflammation spreads up the ureters, all the more quickly because they are dilated and open into the lowest part of the bladder; the pelvis of the kidney becomes involved; the septic organisms spread into the medulla and cortex; minute abscesses form in all directions; the intense congestion interferes with the secretion of urine (all the more readily because the substance of the kidney is hard and indurated) and partial or even complete suppression of urine is added to septic absorption.

Hitherto this last form of urinary fever has been assigned to the shock caused by the complete evacuation of the residual urine affecting injuriously the already hardened and degenerated kidneys. The symptoms, however, the mode of onset, and the pathological changes are entirely different from those that are met with in shock. The shock that follows operations upon the urinary organs is always immediate in its action, whether it affects the whole nervous system, or only those parts of it that are in close physiological relation with the nerves of the injured structures. On the other hand the troubles that are caused by the evacuation of residual urine (except in those cases in which syncope has been caused by suddenly drawing off a large amount from an old and feeble patient while standing upright) never begin at once. There is an interval, always of some days, sometimes of more than a week; and when they do set in they begin, not suddenly, but quietly and insidiously. They are certainly, therefore, not due to shock.

It is equally true that they are not the result of renal congestion and suppression of urine. In many instances the secretion of urine is not interfered with at all. In others, so far from there being suppression, there is exactly the opposite, polyuria. I have met with this on several occasions. In one instance under my care the amount rose to between seven and eight pints in the twenty-four hours. The symptoms, moreover, that are present in these cases bear but a very remote resemblance to those of true suppres. 
sion, such as that which is caused by obstruction of the ureters. Total suppression of urine may continue for ten or even fifteen days before it proves fatal. In many cases there are no symptoms at all for the first seven or eight. When they do set in, there is no continued fever, or rigors, or. delirium. Failure of muscular power and weakness of pulse are the characteristic features. Consciousness is retained to the last. I admit that when there is partial suppression the symptoms are often aggravated and complicated by it. Naturally if the toxins that are absorbed from the urinary passages are not excreted by the kidneys they accumulate in the blood, together with other deleterious substances that should be got rid of in the same way, and produce a proportionately greater effect. But such a cause, acting in such a way, must be regarded as entirely secondary. On the other hand, the symptoms that occur in these cases are identical in every respect with those that follow operations performed upon the same kind of patient, if the wounds become septic. The gradual onset, the condition of the pulse and tongue, the delirium at night, and the steadily advancing weakness without any marked degree of fever are exactly what is met with in the so-called exhaustion that proves the end of so many septic operations in the aged. The symptoms in short are those of slow septic poisoning; and they are due to the gradual absorption of the toxic products of the organisms growing in the urine and on the walls of the urinary passages. They may be modified by the failure in the excretory power of the kidneys, which prevents the system getting rid of the poisons as rapidly as it should, but the essential cause is the absorption.

There are of course accessory agencies. Chronic interstitial nephritis, for example, is the rule when there is much residual urine. In most instances at the time when catheter life is commenced it is already far advanced. The general health, therefore, is impaired, and the excretion of waste products imperfectly carried out. But the broad fact remains that this form of urinary fever does not occur unless catheters are introduced (it does not for instance occur after aspiration); it begins within a few days of their introduction; it is worse in proportion to the amount of residual urine 
contained in the bladder; and it never breaks out unless and until this becomes infected by septic organisms.

The frequency with which the catheter has to be passed depends upon what is found.

If retention has been complete the catheter must be passed at regular intervals, three, or preferably four times in the twenty-four hours according to the capacity of the bladder, until the balance is restored and the bladder can empty itself again. Meanwhile every endeavour must be made by general and local treatment to diminish the obstruction and increase the power. Recovery may be perfect even after months have passed. Much more frequently some power is regained, but the condition never becomes quite so good as it was before.

If there has not been complete retention, but a certain amount of residual urine is found, the frequency depends upon the quantity. For three ounces the catheter should be passed once a day, preferably at bedtime, so as to secure as long a rest as possible ; twice for six, and then once more for every additional two ounces.

This must be continued for the rest of life. Such a condition cannot improve; it is more than probable that it will grow steadily and perhaps rapidly worse.

Treated in this way the power of the bladder grows weaker and weaker. The muscles are not used, and waste; the amount of residual urine increases, and the catheter has to be passed at shorter intervals. The treatment relieves the symptoms for the moment, and makes the consequences worse.

Sooner or later the constant passage of an instrument leads to congestion and irritability of the neck of the bladder. Micturition becomes more frequent, and at last it may be almost incessant, so that there is no rest day or night.

Sooner or later, as the gland continues to enlarge, the introduction of the catheter, though at first it may have been perfectly easy, becomes more difficult, until the patient in many cases is unable to pass it himself. When congestion supervenes, as it always does owing to the increased straining and the difficult instrumentation, it may be almost impossible.

Sooner or later the use of the catheter leads to cystitis. It 
is true that some patients appear to enjoy immunity, and that by dint of ceaseless care its occurrence may be retarded in all. But as the call becomes more urgent and more frequent, and as the patient grows older and less able to help himself, septic inflammation is certain to occur if a catheter is habitually used.

Taking these facts into consideration it must be admitted that the treatment of enlargement of the prostate by catheters leaves a great deal to be desired. The beginning of catheter life is a time of very great risk even when the kidneys are sound. When they are not sound or when there is a large amount of residual urine, the danger is graver still, though, it may, it is true, be diminished to some extent by the most exact attention to the principles of aseptic surgery. And when this difficulty has been overcome, treatment by catheter (as carried out by the patient) involves of necessity progressive deterioration of the function of the bladder, and a rapidly increasing liability to septic cystitis and pyelonephritis.

It is perfectly true, as Mr. Buckston Browne, amongst others, has pointed out (Med. Soc., March, I893), that there are numerous instances all round us of elderly men, active in all the pursuits of ordinary life, and many of them exceptionally highly placed in the conduct of affairs, who are obliged to pass all their urine by catheter, and who lead comfortable, useful, and happy lives.

But there are many others, and I have no hesitation in saying a very much larger number, of whom, from the very nature of things, much less is heard. The successful ones are living and always in evidence before us, and this alone is apt to lead us unconsciously to over-estimate their number.

As Mr. Buckston Browne admits this state of comfort is dependent in the first place upon there being only a moderate amount of intravesical prostatic growth; and, secondly, upon the attention paid by the patient to his condition. They must, therefore, of necessity belong to those classes which have the knowledge and the means for taking precautions.

Even under these fortunate circumstances there comes a time when the power of personal attention fails; and then, unless they can afford every luxury, and sometimes even when they can, septic cystitis sets in at last. 
While I am prepared to agree with Professor White "that in patients with but moderate obstruction, or with a high degree of compensatory hypertrophy of the bladder, with a small amount of residual urine which remains sterile, and in whom catheterism is easy and painless, operation is not to be thought of," I am sure that it is no less essential to bear in mind that the habitual use of catheters is in no respect a cure for the complaint. It only relieves one of the symptoms, and that not perfectly, and only for a time. It always leads to atony and irritability of the bladder, and nearly always to cystitis. As the disease advances, instruments have to be used more frequently. However easy it is at the first to introduce them, it always becomes more difficult as the need for them grows greater. And though many men lead comfortable and happy lives, they are not the majority, or even a large proportion, when all classes are taken together. Moreover, the end at last, even in them, is the same.

The comparative comfort so often (but not always) seen at the beginning of catheter life is very apt to throw into the background the much darker picture of what the future will be.

\section{The Prevention of Irritability of the Bladder.}

Irritability of the bladder and increased frequency of micturition are among the earliest and most constant symptoms of enlargement of the prostate. The mucous membrane at the neck of the bladder becomes congested. Its sensitiveness is increased, and the slightest cause excites the desire for micturition. When this is allowed to continue inflammation soon follows.

Passive congestion of the vesico-prostatic plexus is to a great extent natural in old age. The veins enlarge and become varicose; the valves fail to act; the circulation in general becomes more sluggish; the blood finds more difficulty in returning, and the vessels of the pelvic and abdominal viscera are liable to be overloaded. And this is often made a great deal worse by sedentary habits and want of exercise, especially walking, which has a wonderful effect in quickening the circulation through the pelvis. 
In such a condition very little is required to cause an acute attack. A sudden chill; an attack of indigestion; sexual indulgence ; constipation, or any irritation about the rectum; an unusual amount of alcohol; a long railway journey, or prolonged retention, and at once the mucous membrane becomes swollen; there is more difficulty in emptying the bladder, and the desire becomes more and more imperative as the difficulty increases.

Irritability of the bladder without enlargement of the prostate yields readily to general measures. Local treatment is not only not needed, but is to a great extent harmful. Every instrument or foreign substance that comes into contact with the deep part of the urethra is an irritant, and the more they can be avoided the better. The patient should be confined to the house, or his room, or to bed, according to the severity of the attack. The bowels should be kept freely open; warm baths given night and morning; the diet carefully regulated, especially as regards the amount of alcohol ; and especial attention paid to the condition of the urine. Alkalies are often of great use in these circumstances, the citrate or bicarbonate of potash, or liquor potassæe well diluted and given in barley water. These may be combined with full doses of hyoscyamus or of conium. Nothing is so irritating when the neck of the bladder is congested and tender as an excess of uric acid or oxalate of lime in the urine. Even sudden changes in the specific gravity, or reaction, are of consequence. If the irritability is so great as to prevent the patient getting any rest a morphia suppository may be given at night time, or a hypodermic injection. I have not found any benefit from either heat or cold in the rectum, while the application of either is nearly always very repugnant to a patient's feelings.

When, however, the prostate is enlarged, general measures such as these are very rarely sufficient of themselves. The presence of residual urine and the straining and effort caused by the disadvantage at which the muscular coat of the bladder is placed do not allow the congestion to subside. A vicious circle is established, the irritability causing increased frequency, and the increased frequency making the congestion and irritability worse. All instrumentation is 
injurious, but local measures must be employed to give the bladder a fair chance of rest and recovery.

A slight degree of irritability can be relieved by passing full-sized steel sounds and leaving them for half-an-hour or more. The superficial vessels are emptied by their pressure, and though they fill again as soon as the sound is withdrawn it is of some advantage to have enabled their walls to contract, if only for a time. The dilatation has also a very considerable effect upon the muscular fibres that surround the lower part of the bladder and the prostatic urethra. They are made to tire themselves out and relax. After all sources of irritation have been removed these fibres often maintain a state of spasm which perpetuates itself by the congestion which it causes and the pressure it exerts upon the swollen and tender mucous membrane. In some cases they become hypertrophied, so that at last a kind of sphincter is developed. Sedatives and astringents are of no avail. Dilatation, on the other hand, soon gives relief. The benefit that has been derived from digital exploration of the bladder is in many instances due to this as much as to the drainage that has followed it. The fibres around the neck have been paralyzed for a while, the blood-vessels and nerve endings have been allowed to rest, and by the time the muscles have regained their tone the symptoms have subsided.

If this does not succeed and the irritability continues in spite of alkalies and other internal remedies, relief can generally be obtained by means of injections. Cocaine alone is of little or no use. It may relieve the symptoms for the moment, but owing to the vasomotor paralysis that follows, it nearly always makes the congestion worse, and it may cause bleeding. With the addition of a few drops of adrenalin, however, it sometimes answers very well. Morphia, if advisable in other respects, may be given by the rectum, but it is no use injecting it into the bladder, as it is not absorbed so long as the mucous surface is sound. Guaiacol, dissolved in sterilised olive oil, which is sometimes very beneficial in cases of tuberculous disease, has not succeeded in my hands, probably for the same reason. And the same may be said of antipyrin. Nitrate of silver, however, can nearly always be relied upon. It should be given by what Guyon has 
called the method of instillation-a few drops of a $2 \%$ solution slowly injected into the neck through one of Guyon's catheters after the bladder has been thoroughly washed out and emptied. It is generally believed that in these cases there is an abrasion at the neck of the bladder, or some fissure or small ulcer concealed between the folds of the mucous membrane at the orifice; and that the nitrate of silver acts as an astringent or by coating the surface over with a film which protects it and gives it rest. But as a rule the salt is decomposed by the chlorides that are present before it reaches the surface of the sore, especially as in these cases the neck of the bladder is in a state of spasmodic contraction, and it is more probable that the benefit is due to the antiseptic action of the silver salts. Protargol, half per cent. solution, is even better, as it causes less irritation, while it appears to be quite as powerful as an antiseptic.

Unless there is an intra-vesical prostatic upgrowth, which is a much more common cause of irritability of the bladder than is usually believed, this, combined with the general treatment which has been detailed already, will give relief; the frequency of micturition diminishes; the pain and spasm at the neck subside; and the bladder, to all appearance, regains its former condition. Leaving aside, however, the question of intra-vesical growths (which alters everything, for the irritability they cause can only be removed by operation), relapse sooner or later is certain.

The symptoms are relieved it is true, but the causes are left. They have not been touched. On the contrary, they are able to work at greater advantage. The amount of residual urine is larger than it was before, the catheter has to be introduced more frequently, the mucous membrane is more sensitive, and the condition of the tissues is distinctly changed for the worse. In youth an attack of irritability of the bladder may pass off and leave no ill-effect behind. "It is different when the prostate is enlarged. The blood vessels do not recover their tone so readily, the tendency to congestion is greater, the nutrition of the muscular coat is not so good, and the fibres never regain the whole of their former power. The amount of residual urine is always greater after one of these attacks. 
The more frequently the bladder is attacked the more susceptible it becomes. The intervals grow shorter and shorter; the irritability does not wholly subside; the catheter has to be passed more and more often, and at last the patient's condition becomes one of intense misery. The catheter is required every hour. The urgency is such that the patient cannot endure a moment's delay. The introduction becomes more and more difficult, and the pain, when at last the instrument does pass, is scarcely less than that which comes on as soon as it is withdrawn.

As a rule, long before matters have reached this point, septic inflammation breaks out. Then the downward progress becomes more rapid still. The urine becomes loaded with pus, which collects in the lowest part of the bladder, and is either the last to come out, or never comes out at all. The pain is much more severe and continuous. There is tenderness on pressure over the pubes, and an aching sensation, or if the attack is acute, throbbing in the perineum, spreading round the loins and down the thighs. Micturition is incessant. Every attempt is followed by blood, as the muscles around the neck of the bladder contract and squeeze the swollen and congested mucous membrane. The patient is hot and feverish; his tongue is dry; the appetite is lost ; rest at night is hopeless; and he is absolutely worn out by the intense burning pain that forces him to try to pass water or to introduce a catheter every minute, and grows worse and worse with every attempt. 
CHAPTER IX.

\section{The Local Treatment of Enlargement of the Prostate.}

Its Complication-Cystitis-Retention-Hæmaturia and Calculus.

THE outbreak of cystitis marks a distinct stage in the course of enlargement of the prostate. It means that the protection of the mucous membrane has broken down.

With very few exceptions the infective organisms which are carried into the bladder by catheters, or succeed in entering in other ways, are incapable of harm, so long as the mucous membrane remains healthy and uninjured. If, however, it sustains the least hurt, even if its power of resistance is merely lowered by cold or an attack of congestion, the organisms are at once enabled to gain the upper hand, inflammation sets in, and unless the attack is very slight and soon brought to an end, the deeper structures become involved; the organisms invade the submucous and the muscular layers; the toxic products that are formed in the urine and in the walls of the bladder are absorbed, and fever and septic poisoning follow as a matter of course. Every attack of cystitis in a case of enlargement of the prostate helps to weaken the power of the bladder and affects more or less seriously the health of the patient.

There are various ways in which septic organisms gain entrance into the bladder. They can pass into the urine through the kidneys. It has been proved of many kinds of micro-organisms that they are excreted in this way, at least in part; and they have been cultivated from the urine. They can also pass into the bladder from the neighbouring bowel. This has been proved experimentally in the case of animals, and many clinical observations, such as the frequency with which cystitis occurs after operations for hæmorrhoids, render 
it highly probable in the case of man. But it is not likely that either of these is the ordinary route. In the majority of cases of enlargement of the prostate germs are introduced directly by means of catheters. The frequency with which septic cystitis occurs is proportionate to the frequency with which catheters are used. In the very few cases in which it breaks out independently of catheterization, the infection probably does not occur through the kidneys, but by direct extension along the mucous membrane of the urethra, or from the neighbouring bowel. Infection, in other words, is almost always of local origin, and caused by the very means that are employed to keep its consequences in check.

If everything were ideally perfect this complication would not occur. It ought not to occur, and by taking the greatest precautions it can, in most instances, be postponed. But when a catheter has to be passed every day, and often several times a day, and that for years together, over a mucous surface that is exceedingly sensitive, and in many instances becomes more sensitive every time it is touched, it is not in human nature to maintain a sufficient degree of care. Septic cystitis, sooner or later, is the rule.

If the attack is at all severe the patient must be confined to bed. Warm hip baths should be given night and morning, the bowels kept open, and the diet carefully regulated. Alcohol is not good for cystitis, but the patient may require it. Digestion is rarely very active; sleep is always much disturbed; the pain is intense and incessant; and the action of the heart often feeble and inclined to intermit. The affection does not long remain a local one. Septic intoxication, due to absorption through the inflamed vesical wall, is soon added to the other troubles.

Urotropin, salol, boric acid, quinine, the acid phosphate of soda, and benzoate of ammonia, are the most efficient internal remedies; but the condition of the patient must not be lost sight of in attending to the state of the urine. More good may be done sometimes by small doses of alkalies with stomachics and mild tonics.

Sedatives are nearly always required. The best is opium, either by the mouth or the rectum, if, that is to say, the condition of the kidneys will allow it. It is the only drug that 
can be relied upon to relieve strangury and save the patient's strength. The liquor Opii sedativus is the preparation I always prefer. If this is not well borne, nepenthe may be given, or morphia hypodernically. And if these are not satisfactory, or are contra-indicated by the state of the kidneys, recourse must be had to chloral and bromide; but though these procure sleep, they do not give the same relief. The same may be said of sulphonal and trional, which often answer for a time. Sometimes when nothing is very satisfactory a mixture of small quantities of many hypnotics, opium, chloral, bromide and cannabis indica succeeds.

The local treatment of cystitis must be guided by the extent of the pathological changes in the bladder and prostate. The principle is always the same. Septic organisms have found their way into the bladder. The residual urine that lies in general almost inert in the lowest part of the cavity has been transformed into a virulent poison which cannot be expelled. The growth of the septic organisms must be arrested and the bladder freed from the irritant that is destroying it.

In the simplest cases, when the attack is recent and the walls of the bladder are not as yet seriously involved, and . when a catheter can be passed without much difficulty, the growth can be checked by washing out the bladder at regular intervals, and emptying it as thoroughly as possible of all residual urine.

The simplest apparatus is a glass funnel, with a long piece of rubber tubing, and a catheter provided with a terminal opening. The catheter should be made with a side branch shut off by means of a stopcock, and this should be so arranged that while the fluid can pass down from the tubing into the bladder it will not pass back again, but flow out from the bladder through the side branch.

The residual urine is drawn off first, the apparatus filled, and to prevent cooling, immersed in the fluid at the desired temperature. The catheter is then passed down, just through the membranous part, the stopcock turned, and the funnel raised as high as necessary. A few inches pressure is usually enough. Then the stopcock is reversed, and the fluid allowed to escape through the side branch, so that the same does not 
enter again. As a rule the quantity should not exceed four or six ounces. Finally the catheter is pushed into the bladder, and the cavity emptied.

Hydrostatic pressure for washing out the bladder has many advantages over a syringe. It can be graduated to a nicety, and is perfectly steady. The patient can regulate it himself, directing the stream according to his own sensations, and stopping it when it begins to cause the least discomfort. And the quantity that is injected can be easily checked against that which flows out. This is necessary, not only because of sacculi, but because sometimes there is a valvular growth hanging over the orifice, so that fluid can enter readily without being able to return.

In many patients the bladder can be washed out without passing a catheter beyond the fossa navicularis. The resistance of the sphincter is overcome by gradually increasing the pressure. This is not advisable in cases of enlargement of the prostate, unless it is wished to exercise the muscular power of the detrusor urinæ. The end of the catheter should be passed through the sphincter at the apex of the gland, so that the fluid can return without throwing any work upon the muscular coat.

Belfield (loc. cit.) recommends that when the bladder is to be washed out thoroughly the patient should be placed in the knee and elbow position, or that suction by means of a syphon should be employed. This, however, is only necessary when there is a great accumulation of debris in the postprostatic pouch, or when the interior of the bladder is very irregular in shape. Very great care is required when there is any reason to suspect the presence of sacculi. Their walls are often exceedingly thin; fluid enters easily, but there is no force to drive it out again.

A great variety of solutions have been recommended at different times for washing out the bladder. For purely cleansing purposes sodium chloride and boric acid dissolved in a solution of sodium borate are the most useful. The former dissolves away any deposit of pus rather the more easily. The latter has some though a very slight degree of antiseptic power. The sodium borate allows rather a large amount of boric acid to be held in solution. 
More powerful antiseptics are required in most cases. The best germicides, so far as the bladder is concerned, are nitrate of silver, one part in a thousand, and corrosive sublimate, one part in two thousand. I prefer the former, as it is less painful, and equally efficacious. The citrate of silver which has been recently recommended as less irritating is not so soluble. Silver salts are of course decomposed at once, but though the chloride is insoluble, it appears to possess an exceptional faculty for preventing the growth of the bacillus coli.

Iodine terchloride (one part in two thousand) which is spoken of very highly by Belfield, I believe to be the next best. Carbolic acid of sufficient strength to act as a germicide causes too much irritation. Quinine, boric acid and naphthol check the growth of organisms, but so far at least as concerns the urine in the bladder, do not appear to possess the power of destroying them. Iodoform is of no use in suppurative cystitis ; and nitric and phosphoric acids which have been recommended for the purpose of dissolving the concretions that form upon the walls, have little or no power under these conditions. If the organisms that cause the decomposition of urea are destroyed, the formation of the ammoniomagnesian phosphate ceases, and that which has been deposited already is thrown off by the epithelial cells as they are renewed from below. After orchidectomy I have many times noticed masses of phosphatic concretion that were previously firmly adherent to the mucous surface, separate themselves spontaneously as the wall recovered and come away with the urine.

Sanitas, boroglyceride, sulpho-carbolate of soda, salicylic acid, salicylate of soda, salts of bismuth and of lead, and many other substances have been recommended from time to time, and may occasionally be of use. But I do not consider them so certain or so efficacious as nitrate of silver and corrosive sublimate and they are not less painful.

In many cases, especially when the inflammation is acute, washing out the bladder is not well borne and is very painful. In these Guyon's instillation method may be advantageously employed instead. The bladder must first be emptied completely and then about twenty drops of a two per cent. 
solution of nitrate of silver, or of a one-half per cent. solution of protargol slowly injected through one of Guyon's catheters into the neck and the prostatic portion of the urethra and left there. This method of applying an antiseptic has the merit of never causing any tension, so that it may be used in the most acute cases of cystitis and even where sacculi are present. It enables a germicide of much greater strength than could otherwise be employed, to be applied directly to the mucous surface. And it deals with that part of the bladder in which inflammation nearly always begins and which, physiologically speaking, is far more active and inportant than the rest.

Provided the cystitis is recent, the condition of the wall of the bladder in other respects good, and the introduction of a catheter easy, septic cystitis can generally be checked and brought under conţrol by adopting sufficiently energetic measures of this kind. The patient is restored to a state of comparative comfort, but he will have to use a catheter more frequently than he did before; and as the same causes continue to work, and to work from a point of greater advantage than they did, he will be exposed to the old risks in an even greater degree. The consequences have been averted; the causes have not been removed; recurrence is only a matter of time, and each time recurrence takes place the intervals grow shorter and the symptoms more severe.

It seldom happens, however, when septic cystitis occurs in a case of enlargement of the prostate that the conditions are so simple and straightforward; and if they are at the first they never remain so for long. The patient in only too many cases is suffering from incipient uræmia and septic intoxication, and is broken down in health and strength from pain and want of rest. The bladder is often in a hopeless state, ulcerated, coated over with a foul smelling mixture of phosphates, pus, and decomposing urine, and most irregular in shape. A catheter is required every hour or even every halfhour. The prostate has grown to such an extent, or has become so swollen from congestion, that it is difficult for the surgeon and impossible for the patient to pass one. And every attempt is attended with hæmorrhage and agonizing pain. Catheterism under such conditions has been aptly described as a slow but fatal form of torture. 
The indications are the same. The bladder must be emptied and the growth of the septic organisms stopped, either by employing germicides, or by depriving the germs ${ }^{\circ}$ of the food upon which they thrive, or by both. Microorganisms are not capable of much harm in a bladder that is kept empty.

If the muscular coat still retains sufficient tone, so that when the resistance in front is reduced to a minimum the bladder can keep itself empty, the simple expedient of tying a catheter into the urethra may be tried. The catheter must be a flexible one, and only carried in just so far that the eye lies inside the neck. The urine should flow from it drop by drop, without any break, as it comes from the ureters. If when the catheter is moved either backwards or forwards, a small stream flows out, the eye of the catheter is in the wrong place and the drainage is not perfect.

This method has, however, many drawbacks. The catheter is most difficult to keep accurately adjusted. It is no good as a drain when the middle lobe of the prostate is enlarged, for the orifice of the bladder is no longer the lowest point; and it is no good when the walls of the bladder are rigid, so that they cannot contract, or when they are toneless and flaccid, or when there is a large and thin post-prostatic pouch. The walls collapse and fall together, instead of contracting uniformly towards the orifice, and the recesses remain unemptied. And in many instances tying a catheter into the urethra gives rise to additional troubles of its own. I have more than once known suppression of urine caused by it. No matter how soft the instrument may be nor how often it is changed, its presence nearly always leads in the course of a few days to inflammation of the urethra, and this may spread upwards to the bladder, or to the epididymis, or to the veins of the prostatic plexus. Add to this that tying in a catheter can never be of use except as a temporary measure, for a few days at the most; and I think it will be admitted that such a proceeding is a most ineffectual remedy, when septic cystitis is associated with enlargement of the prostate, unless the attack is very recent and slight, and the enlargement very small.

When drainage of the bladder is required in a case of 
advanced cystitis associated with enlargement of the prostate, the method that is selected must be one that is capable of being continued for as long as may be wished, and if necessary, of being made permanent. Relapse, when once the bladder and prostate have reached this stage, is practically certain. If radical measures are not sanctioned, or if it is too late for them, it is better for the patient to maintain a permanent system of artificial drainage than to allow the opening to close after the immediate effect has been obtained, and resort to the use of the catheter again. If septic cystitis was not prevented before it will not be prevented in the future. The liability increases with each' attack, and each attack increases the risk of pyelonephritis.

The choice lies between the two routes, over the pubes, and through the perineum, either opening the urethra at the apex of the prostate and introducing a soft rubber tube into the bladder through the prostatic portion of the urethra, or pushing a trocar and cannula directly through the perineum and the prostate, into the post-prostatic pouch. Of these two I am decidedly in favour of the former. The only exception is when the bladder is very much contracted with rigid unyielding walls, or when the patient is already at death's door, in a state of incipient uræmia, worn out by want of sleep and the torture of hourly catheterism, and poisoned by septic absorption from the bladder, so that only the least. possible may be done.

It is true that a supra-pubic opening is in the front of the bladder, and not at its lowest point, so that it might be thought that it would not act so well; but as a matter of fact the pressure of the abdominal viscera, especially when the patient is sitting up, as many of these old men must, almost obliterates the cavity of the bladder, if that is to say the outlet is free, and effectually prevents the collection of stagnant urine at the bottom.

The opening into the bladder may be made either with a trocar and cannula or by dissection. Of these two methods the former, although it answers well in cases of retention from stricture, and is much the more simple, is not advisable for drainage when the prostate is enlarged. The bladder in many cases is small and contracted. The peritoneal fold 
may descend as low as the pubic symphysis. A cannula is often not well borne when there is an intra-vesical growth, and without a cannula sufficiently long to dip down into it, it is not easy to drain a large and deep post-prostatic pouch. And a calculus would either be missed altogether, or if found cannot be removed.

On the other hand, through a suprapubic incision the interior of the bladder can be examined thoroughly in every part. Pouches, if any are present, can be drained. Calculi which are much more common in cases of septic cystitis associated with enlargement of the prostate than is usually believed, can be removed. The condition of the intra-vesical portion of the prostatic growth can be accurately made out, and later, when the patient has recovered, and the tissues around the wound are consolidated, and the urine is no longer septic, the projecting mass can be removed without the necessity for making another incision. The opening can be maintained permanently, if this is desired (it is practically impossible to devise a perineal drainage tube which will allow the patient to sit with any degree of comfort), and by anchoring the bladder to the posterior surface of the abdominal wall, and raising it in this way, the sagging downwards of the posterior portion of the bladder and the formation of a deep post-prostatic pouch can be to a great extent prevented.

The operation, which was first recommended for this purpose by $\operatorname{Sir} \mathrm{H}$. Thompson, requires the usual precautions. The day before the skin over the pubes is shaved, thoroughly well scrubbed with soap and water, and covered with a carbolic compress. The bladder is emptied and well washed out with a warm solution of boracic acid (half an ounce to the pint) before beginning the operation. It is probably hopeless trying to disinfect the walls in a case of advanced cystitis, but care should be taken that the contents are as aseptic as possible. Sufficient is left in to fill the bladder to moderation. As a rule eight ounces are enough, but if the size is small, or if there is any reason to suspect the presence of sacculi, this must be reduced by one-half. Distension of the rectum I have quite abandoned. It tends, it is true, to raise and fix the floor of the bladder, but where the question is merely one 
of drainage this is unnecessary, and it is by no means devoid of danger.

Trendelenburg's position may be adopted with advantage if the patient's respiration is good, but great care is required in old men with rigid chests and emphysematous lungs.

The incision should be in the middle line, from two and a half to three inches in length, commencing over the symphysis. Trendelenburg's transverse division has nothing to recommend it, and is followed more frequently by weakness of the wall. The tendinous structures are divided freely down to the transversalis fascia. As soon as this has been opened the finger is introduced and the sub-peritoneal fat and peritoneum carefully lifted off the bladder wall and hooked upwards with the finger nail. If the bladder is tense and prominent it may be punctured at once with a scalpel, and the finger introduced behind it to hook up the wall and. examine the interior. If, on the other hand, it is deeply situated a tenaculum should be fixed in it to hold it more firmly. The incision should be in the median line on the most prominent part of the bladder below the peritoneal fold, between the veins that lie upon its anterior wall, and long enough to admit the forefinger without much difficulty.

If there is nothing that requires removal there is no need to insert any sutures in the bladder wall. A soft rubber drainage tube of full size should be introduced well into the cavity and fixed there, either by means of a suture passed through the skin, or preferably by a plate which slides up and down upon it, and can be secured by strapping. The other end can be conducted to a ressel under the bed. The upper part of the incision is then drawn together by sutures and soft absorbent dressings applied. In this way the patient can be kept perfectly dry, the bladder washed out as often as required either through the urethra or through the tube, and at the end of three days, when the soft tissues around the wound have become consolidated, the tube can be removed and replaced or not according to the condition of the bladder.

If, however, anything is found in the bladder likely to require manipulation, if, for example, the mucous membrane is coated over with dense phosphatic concretions, or if it is 
thought that it might be advisable to remove the obstructing prostate at a subsequent operation, when the urine is no longer septic, two sutures should be passed through the muscular wall of the bladder, one on each side, to hold it up and prevent any strain falling upon the soft cellular tissue around it. Afterwards they can be withdrawn.

Many attempts have been made to utilize a supra-pubic channel obtained in this way as a permanent urethra, under the control of the patient, so as to do away for ever with the necessity for passing a catheter.

One of the simplest consists of a soft rubber catheter held in the sinus by means of a silver plate, which is kept in position with a belt and a perineal band. The other end of the catheter is conducted by means of a rubber tube to a portable urinal strapped on to the patient's leg, or supported from the belt.

In a few cases I have used a vulcanite and rubber tube, provided with a stopcock, without any apparatus to collect the urine. Every three or four hours the patient obtains relief by turning the stopcock and allowing the retained urine to escape under the abdominal pressure. There is very little leakage around the tube, and this can be prevented by adopting Wiley Broome's suggestion and cutting a screw thread on its outer surface.

Hunter McGuire (Trans. Amer. Surg. Assoc., I89o) recommends opening the bladder at the lowest a vailable median point, and leaving open the upper end of the abdominal wound, the rest of the abdominal incision being sutured. This leaves a fistula two or three inches in length, the walls of which are kept in apposition by the pressure of the viscera, so that the patient can retain his urine or pass it at will. Dr. McGuire has written to me since, that he does not attach the bladder to the abdominal wall in any way because this interferes with the length of the artificial urethra. "I find it necessary to keep a plug of aluminium or of some other metal in the sinus, the patient taking it out only when he wants to pass water."

The artificial urethra in this operation is surrounded by cicatricial tissue, and is liable to contract. With the view of preventing this Morris (New York Medical Fournal, July, I8go) 
has tried to line the channel with skin flaps. "A ribbon of skin and subcutaneous tissue about three inches long and one-third of an inch broad is dissected away from either side of the abdominal incision, leaving the caudal extremities of the ribion attached. The free ends of the ribbons are now sutured with fine catgut to the mucous membrane of the bladder, each on its respective side. The temporary sutures which held the bladder up are now cut, and as the bladder drops down it takes with it the two ribbons of skin, which lie prettily face to face, and which are to form a soft urethra of skin. A short rubber drainage tube is left in the bladder for forty-eight hours. Four weeks after the operation the ribbons of skin form a soft round urethra. The patient can retain his urine or pass it at will, and he substitutes a glass drainage tube for the penis, pressing one end of the tube against the supra-pubic meatus and passing urine through it. In order to do this he makes an ordinary expulsive effort and no urine comes by way of the penis." Dr. Morris has written to me since that the sinus became rather too small from contraction of the walls, and that in another case he would make the flaps wider.

In one case under my care I tried this method with this alteration, that I left the ribbons attached by their upper ends, in order that I might secure at the same time the advantage of Hunter McGuire's long valvular urethra. The urethra answered very well for a time, but an angle developed in it where the cutaneous flaps joined the mucous ones. The superficial portion of the sinus ran upwards towards the head; the deep part backwards towards the sacrum, and some phosphatic material collected at the bend. Later a perineal abscess formed (the original operation was performed for sloughing of the urethra consequent upon exceedingly severe extravasation of urine), and when this was opened the urine came through it, so that the supra-pubic channel was no longer used, and was allowed to close. The skin flaps marked its external opening merely as a small dimple. Had a plug or a tube with a stopcock been worn in it at night time I believe it would have remained straight and free, and would have answered admirably. 


\section{Retention.}

Retention of urine is one of the most common complications of enlargement of the prostate. Sometimes it occurs suddenly, without warning, and is the first intimation the patient receives that there is anything wrong. More often there has been gradually increasing difficulty for months or years before. In either case no drop of urine can pass; the bladder becomes distended more and more; there is the most intense pain in the lower part of the abdomen ; and unless speedy relief is given symptoms of a typhoid character quickly supervene.

This condition must be distinguished from the simple accumulation of residual urine: In the one the orifice of the bladder is closed, and no power that the muscular coat possesses is of the least avail. In the other the orifice is free, more or less, and the cavity full because the muscular fibres are unable to expel the contents.

Retention may occur in every stage of enlargement. The immediate cause is nearly always an attack of congestion. The route may be perfectly free under ordinary circumstances, or it may be half closed by a valve hanging over the orifice, or by the growth of the prostate around it. Suddenly congestion sets in; the mucous membrane becomes enormously thickened and swollen, and the size of the valve is increased, or the width of the passage is narrowed to such an extent that no urine can flow down. The more the patient strains the worse the congestion becomes, and at last the mucous membrane is engorged with blood to such an extent that it is as hard and rigid as the prostate that lies around it, and the sides of the urethra fit into each other as closely as if they were dovetailed together.

Sometimes this congestion is inflammatory, caused by injury, the passage of catheters, for example, or the impaction of a calculus. More often it is passive, arising from a sudden chill, exposure to cold or wet, or alcoholic or other excess.

In some of these cases the bladder attains an enormous size, reaching nearly to the umbilicus, holding three or four pints of fluid, and causing a distinct and characteristic pro- 
jection of the lower part of the abdomen, even when the patient is lying on his back in bed. In others, however, and these are often the worst, there is resonance over the pubes. The bladder is so small and its walls so thick and hard that it cannot stretch or hold more than a few ounces.

Immediate relief is imperative. Retention, in a case of enlarged prostate, for however short a time, never occurs without leaving some serious result behind. Atony from over-stretching of the muscular fibres is invariable, and may be complete' and permanent. More commonly it is only partial; but when it occurs in an old man suffering from a complaint of this kind it rarely passes away entirely. The secretion of urine is checked by the greatly increased pressure in the urinary passages. Hæmaturia is exceedingly common, independently of the passage of instruments. The distended and often varicose veins around the neck of the bladder give way, and their contents pour into the walls and into the cavity through rents in the mucous membrane. Cystitis frequently sets in, partly from the means taken to procure relief, partly because of the injury which has been inflicted upon the mucous membrane. And sometimes the consequences are graver still; suppression and uræmia may follow.

A catheter must be passed at once, without more delay in the preparation of the patient than is absolutely necessary. If this does not succeed the bladder must be tapped.

Retention of urine in a case of stricture of the urethra can nearly always be relieved by placing the patient in a hot bath for a quarter of an hour or twenty minutes, giving him a full dose of opium and getting the bowels to act freely. The urine may not come in a stream, but it will come sufficiently fast and in a sufficient quantity to relieve the urgency. When retention is due to enlargement of the prostate this is rarely advisable.

For one thing time is too precious. The bladder, in a case of stricture, has nearly always had difficulty for a long time past, and a few drops of urine usually manage to escape, often continuously. Retention due to enlarged prostate (not, of course, the mere accumulation of residual urine) is complete and urgent. The congestion, too, which is nearly always the immediate cause, is passive. Unlike the inflam- 
matory hyperæmia which sets in around a stricture that has been irritated, it is but little relieved by warmth, and may be made worse. Opium again, unless the condition of the patient's kidneys is known beforehand, cannot be given, for the dose, to be of any immediate benefit, should be a full one. The wall of the bladder is weaker and much nearer atony, and the patient is not a young man.

It is best for the patient to lie down. An aseptic coude catheter, No. 9 or io English, is the most suitable to begin with. If this does not answer a gum elastic with a stilet or a metal one should be tried. The point of the instrument in either case can be tilted so as to rise over any obstruction upon the floor of the urethra; but the former has the advantage that the curve can be altered by withdrawing the stilet a little, as originally recommended by Hey.

Metal instruments should be provided with a prolongation curved in the reverse direction to fit into the handle, so as to avoid spirting of the urine over the bed clothes. In the case of a soft one, a piece of ordinary rubber tubing may be attached for the same purpose, but this is, of course, impracticable with gum elastic catheters when a stilet is used.

The greatest possible care must be taken to prevent any septic or pyogenic organisms being carried into the bladder. All the precautions that have been already detailed must be rigidly observed. The hands, the glans penis and the prepuce must be thoroughly cleansed and rendered aseptic. The instruments must have been sterilised and have been kept from all risk of contamination afterwards. And the anterior part of the urethra must be washed out as well as it can be before the catheter is introduced. Drawing off the urine in a case of retention due to enlargement of the prostate requires as much care as any other serious surgical operation.

The bladder should be allowed and encouraged to empty itself. I have shown elsewhere, in my work on "Inflammation of the Bladder and Urinary Fever," that the cause of the disasters which not unfrequently follow the treatment of residual urine is not the emptying of the bladder as is usually held, but the introduction of septic organisms into a bladder - which contains a certain amount of urine which it cannot 
expel. Emptying the bladder is a safeguard rather than a danger, but of course it should not be done too suddenly, or with an old and feeble patient standing in an upright position.

Whether the catheter should be tied in or not depends upon the difficulty there has been in introducing it, and upon the state of the urine. It may be taken for granted that unless the attack is a very recent one the catheter will be required again in the course of an hour or two, not because of the amount of urine, but because of the irritability of the neck of the bladder. The mucous membrane in these cases is so engorged with blood and so hard that it is often many days before the swelling undergoes any appreciable diminution and the muscular coat of the bladder recovers. During all this time a catheter must be passed at frequent intervals; and accordingly, even when there is but little difficulty in its introduction, it is usually better to tie it in, at least for the first twenty-four hours.

If the urine is foul and septic already, or if it is thought for any reason that the aseptic precautions which have been taken have not been sufficient, the catheter should be tied in, an injection of a $2 \%$ solution of nitrate of silver or of a $\frac{1}{2} \%$ solution of protargol thrown into the neck of the bladder, and the bladder kept empty for a time.

The quantity of urine that is passed in the twenty-four hours and the specific gravity should be carefully noted for several days.

The patient should be kept in bed, and the temperature of the room well maintained. I have several times known rigors follow slight exposure. The diet should be light and easily digested, not containing too much nitrogenous material, and the bowels kept relaxed. Warm hip-baths, night and morning, conduce to comfort and cleanliness, but the duration should be very short. The question of alcohol must be determined by the condition of the pulse and a knowledge of the patient's previous habits. The exhaustion from pain and prolonged want of rest is usually so great that it can scarcely be dispensed with.

I have known great benefit follow dry-cupping over the loins and the subcutaneous injection of digitalin (one-fiftieth of a grain) in several cases in which the secretion of urine 
was very scanty, and in one in which it was almost suspended for twenty-four hours.

If a catheter cannot be passed an opening must be made in the bladder.

The choice lies between the supra-pubic and the perineal routes. Rectal tapping through the prostate (for in these cases it is neither easy nor safe to go above it) does not present any advantage over other methods, and cannot be used for drainage should this be thought desirable.

The size of the bladder is a material point. If it is small and contracted with hard rigid walls the supra-pubic route is inadmissible, and puncture through the perineum is to be preferred.

Aspiration above the pubes is only to be recommended when it is wished to gain time, to give the patient temporary relief while preparation is being made for more permanent measures. Retention in a case of enlarged prostate is nearly always due to congestion; the mucous membrane lining the neck of the bladder and the prostatic urethra is engorged with blood to such an extent that it becomes perfectly hard. If this is sufficient to prevent a catheter passing it will not subside for many days to come, and aspiration would, therefore, have to be repeated several times a day for a week or more. It is true that this has been done with perfect safety. I have known a bladder aspirated seventeen times without any ill result. But $I$ have also known suppuration take place in the prevesical tissues after the third aspiration, and there can be no question that the risk increases with the repetition. After puncture with an aspirating needle the bladder empties itself through the cannula, and the mucous membrane, as it collapses, alters its relation to the muscular coat, so that when the instrument is withdrawn the opening becomes valvular. Many perforations, however, lead to softening and inflammation of the wall, and at last to leakage. It is impossible to vary the site very much.

In aspiration a puncture is made with a tenotomy knife through the skin immediately above the upper border of the pubes in the middle line. In stout people there is usually a fold of which advantage may be taken. The trocar and cannula are then pushed through the subcutaneous tissues 
and driven smartly downwards and backwards (in the recum. bent position of the patient), without following too closely the posterior surface of the pubes. To prevent any of the contents escaping as the cannula is withdrawn, a few drops of some antiseptic solution should be sent through it when the urine ceases to flow, and care should be taken to keep the finger pressed firmly upon the orifice. The last few drops of urine are often thick and turbid, and a thread composed of pus and septic debris may cling to the lower opening of the cannula and fall off as it is being drawn out through the wall. No anæsthetic is required.

If the relief of retention is the only object in view, and the size of the bladder permits it, supra-pubic tapping with a full sized trocar and cannula is undoubtedly the best. There are manifest objections to puncturing a swollen prostate, surrounded by immensely distended venous plexuses (though I am bound to admit that I have done it on a good many occasions without meeting with any ill result), and suprapubic cystotomy is an unnecessarily severe proceeding unless it is wished to explore the interior of the bladder for calculi, phosphatic crusts, intravesical prostatic growths, and the like, or to drain it afterwards.

\section{HeMATURIA.}

Hæmaturia is of common occurrence in enlargement of the prostate. The amount of blood lost is seldom important, a drop or two at the utmost. It is only on rare occasions that it is sufficient to distend the bladder or cause any anxiety. The mucous membrane which lines the neck of the bladder and the prostatic portion of the urethra is always in a state of congestion, and the passage of an instrument or the mere contraction of the muscular coat is sufficient without presupposing any degree of violence. Nearly always the blood comes with the last few drops or after the instrument is withdrawn. Exceptionally, when one of the varicose veins which are so common under the mucous membrane at the neck of the bladder gives way, the quantity may be more considerable and the blood may be mixed with the urine. 
Soft adenomatous outgrowths at the neck of the bladder or in the prostatic portion of the urethra not unfrequently bleed freely and persistently.

Prostatic retention is nearly always attended by hæmorrhage. The congestion is so great that the thin walled veins give way, and the mucous and submucous layers are filled with extravasated blood, or the sudden relief of pressure when the urine escapes leads to the same result. The amount is rarely serious, considered from the point of view of the loss of blood, although it is not uncommonly sufficient to lead to the formation of coagula in the bladder. I have met with one or two cases in which the hæmorrhage has been very considerable, distending the bladder to its utmost size, causing the most intense pain, and leading to suppression.

Hæmorrhage from an enlarged prostate seldom requires local treatment. Nearly always as the fluid collects the pressure closes the openings through which the blood escapes, so that they become sealed.

Pain should be relieved by opium, which has the additional advantage of keeping the patient quiet and preventing to. some extent the violent spasmodic contraction of the bladder. The best internal hæmostatic is chloride of calcium, which was originally recommended by Wright, given in fifteen grain doses, twice, with an interval of three hours. This has superseded all other drugs, such as tannic and gallic acids, acetate of lead, sulphuric acid, turpentine, \&c., which used to be given for this purpose.

Ice may be applied over the pubes, against the perineum, and in the rectum, but the application must be continuous and kept up for some hours to have any effect, and this is. rarely advisable in the case of an old man.

If a catheter must be passed for other reasons (such as the relief of retention) advantage may be taken of the opportunity, supposing the hæmorrhage continues, to wash out the interior with cold water, or with a solution of adrenalin, which is far superior to tincture of matico and tincture of hamamelis. But it is rarely necessary to pass a catheter merely for the sake of washing out coagula. They gradually melt down and come away of themselves, taking many days in the process if the hæmorrhage was at all extensive. Of course if 
septic decomposition occurs an aspirator must be passed, or the largest sized catheter that the urethra will admit, and the contents cleared out with the least possible delay.

In those exceptional instances in which the bladder is so enormously distended from a continuance of the hæmorrhage that the condition becomes dangerous, supra-pubic cystotomy should be performed, the coagula cleared out, and the bleeding stopped by the injection of hot water (temp. $110^{\circ} \mathrm{F}$.), or by the actual cautery, or by inserting a catheter in the urethra and plugging the space around it with gauze soaked in a solution of adrenalin. Such extreme measures are rarely required; but clearly if the hæmorrhage continues death will ensue from loss of blood, the intense pain, and the suppression of urine; and no permanent benefit can be gained by passing an aspirator and drawing off some of the contents.

Persistent hæmorrhage should lead to the suspicion of ulceration (which may be malignant) or of a polypoid adenomatous outgrowth. The diagnosis can sometimes be made with the cystoscope; but if this cannot be passed, or if the prostate is of such a shape that much of it cannot be seen, and the bleeding continues in spite of the ordinary remedies, supra-pubic cystotomy should be performed. Digital exploration through the prostatic urethra is only to be preferred when the evidence points to the presence of an outgrowth in the urethra, and not in the bladder.

\section{Calculus.}

Calculus is an exceedingly common complication of enlargement of the prostate, especially after septic cystitis has set in; and it is to be suspected whenever the inflammation is unusually severe and persistent, and resists ordinary methods of treatment. In many cases the diagnosis is of very great difficulty, owing to the way in which the calculi gravitate into the post-prostatic pouch. They never come into contact with the neck of the bladder; do not, therefore, cause the ordinary symptoms, and cannot be reached in the ordinary way. Fortunately they can be detected without difficulty by means of the X-rays, which should always be 
employed if there is the least suspicion of a calculus, or even if the cystitis obstinately resists ordinary measures.

The treatment necessary for their removal does not come within the scope of the present work ; but the frequency with which they occur, especially in the severer forms of cystitis, and the difficulty of diagnosis are additional arguments in favour of supra-pubic cystotomy for this condition, and not mere tapping, either above the pubes or through the perineum. 


\section{CHAPTER X.}

The Radical Treatment of Enlargement of the Prostate. Partial Prostatectomy.

ENLARGEMENT of the prostate is a purely local affection and harmless by itself. The consequences that follow it, the cystitis and pyelonephritis which are so often the cause of death, are due to the obstruction it causes and the congestion that attends it, for without these aids septic organisms would either never enter, or if they did, would be harmless. The radical treatment consists in removing the obstruction, so that these consequences cannot follow.

It is true that many cases, probably two-thirds of those in whom enlargement occurs, do not require treatment of any kind. The obstruction is so slight and the power of the bladder so good that the proper balance is maintained throughout life, and the patient remains unconscious of the existence of the outgrowth until the end.

It is also true that many others who do suffer from it live in a fair degree of comfort, some for the rest of their lives, most for a time, by passing a catheter at more or less frequent intervals, and following out a prescribed régime.

But it is also true that in a very large number the symptoms from the first steadily grow worse. Enlargement of the prostate is a progressive disease, though it is curious how seldom this is realised. Neither the patient nor the gland ever remains stationary. The one is always growing older and feebler, less and less able to help himself; the other is always increasing in size. The difficulty becomes greater and greater while the ability of the patient to deal with it steadily becomes less. Looking at the case month by month, and still more year by year, the change is unmistakable, and always in the same direction. The catheter has to be used more frequently. The irritability of the bladder is greater, and the residual urine more abundant. In such, the end, if 
the same treatment is persisted in, does not admit of doubt. The only way in which the consequences can be averted is to remove the obstruction before the changes it has caused in the bladder and kidneys are too far advanced.

Without going so far as to agree with Prof. Broome when he says that systematic catheterism ought never to be instituted, I cannot help thinking that the dread with which it is regarded by the laity is exceedingly well founded.

There are two quite different ways of dealing with the obstruction. In the one it is enucleated or burnt away with the cautery, and the cicatrisation which ensues is relied upon to prevent subsequent attacks of congestion. In the other an operation is performed upon the testes, or upon some of the structures which are contained in the spermatic cord, with the object of inducing atrophy. If this follows the congestion either ceases spontaneously, or becomes so insignificant that it is incapable of harm.

These methods are based upon such entirely different principles that they must be considered separately before any comparison can be instituted between them.

\section{Partial Prostatectomy.}

A full account of the different operative measures which have been devised for effecting removal or reduction in size of the enlarged prostate was given in a former edition of this work. As, however, many of them are merely of historic interest, and possess no merit which can in any way compensate for the grave disadvantages that attend them, I shall omit them altogether and only deal with those which I have found of practical use.

Supra-pubic prostatectomy or McGill's operation will be described first, as it is one which I have found answers best in the majority of cases in which there is serious obstruction.

\section{Supra-Pubic Prostatectomy.}

This operation, which had already been performed on several occasions by Dittel (Wiener Klin. Woch., July, 1888), 
Trendelenburg (Beiträge zur Klin. Chirurgie, Tübingen, Band viii., 1891), Belfield (New York Medical Record, August, 1886), and Benno Schmidt (Avbeit. aus d. Chiv. Univ. Poliklinik, Leipzig, I886; and Münchener Med. Woch., Feb., I889), first became generally known after the publication of McGill's three cases before the Clinical Society in 1888 . To this and to the subsequent account given at the meeting of the British Medical Association in August, I889, by McGill and his colleagues at the Leeds General Infirmary, this operation owes its present position.

The preliminary steps are the same as those required for supra-pubic cystotomy. The pubes should be shaved, thoroughly scrubbed with soap and water, and covered with a carbolic compress the day before, and an enema given the morning of the operation. As soon as the patient is under the anæsthetic the Bladder is emptied and carefully washed out with warm boracic lotion until the fluid returns perfectly clear. How much should be left in the bladder depends upon what is known beforehand of its size. In most cases it should be sufficient to enable the bladder to be felt when deep pressure is made behind the pubes, that is to say, from eight to ten ounces.

I have long since abandoned the use of a rectal bag. The object of this was not so much to raise the prevesical fold of peritoneum as to steady the prostate during the subsequent manipulation after the bladder has been opened. I prefer the fingers of an assistant whenever one is available. The prostate is forced up better and a much more accurate idea can be obtained as to the amount which requires to be removed. Trendelenburg's position may be adopted if the patient's respiration is sufficiently good when under the anæsthetic, as it renders intravesical manipulation more easy.

The superficial and deep incisions are the same as in supra-pubic cystotomy. The prevesical fold of peritoneum, if it is seen, must be lifted up from off the front of the bladder. Usually it is well out of the way. And the greatest care must be taken to disturb as little as possible the loose cellular tissue and fat which lies between the bladder and the posterior surface of the pubes. 
The opening in the bladder should be made on the most prominent portion of its anterior surface, between the two anterior veins, and should be large enough to admit the forefinger without straining.

The first thing is to ascertain the exact condition of the parts around the orifice, and to determine how the enlargement shall be dealt with. Temporary sutures may be inserted into the bladder wall to hold it up and keep the sides of the incision apart if it seems likely that much manipulation will be required.

McGill advised that the prostate should be removed as far as possible by enucleation with the finger and not by cutting. The larger the prostate the more easily this can be accomplished. By the method of its growth the adenomatous mass pushes all the surrounding structures to one side, and compresses them into an adventitious capsule with which it scarcely retains any connection. The mucous membrane covering the most prominent portion of the growth must be divided, the finger inserted into the wound, and then the central mass quickly separated from the adventitious capsule that surrounds it and shelled out. Hæmorrhage is rarely serious as there are no important vessels divided. Mayo Robson recommends that in cases in which there is a ring of hypertrophied prostate around the internal orifice, a V-shaped piece should be excised from the floor by means of McGill's scissors, a cut being made first on one side and then on the other, and the intermediate portion removed by ring or other forceps. If the lateral lobes are the cause of the difficulty, the index finger can be insinuated within the capsule through the incisions already made, and the masses can be enucleated like small fibroids from a myomatous uterus.

In soft rapidly growing adenomatous prostates enucleation can be accomplished in this way without difficulty. McGill (Brit. Med. Assoc., August, I889) removed both lateral lobes in seven pieces, weighing altogether two ounces thirty grains. Buckston Browne (Clinical Society Trans., May, 1889) twisted away piecemeal by finger and forceps as much as four ounces in weight. White, of Philadelphia (Medical News, Dec., 189o) enucleated eight pieces from the median and lateral lobes, weighing together three ounces. And I have done as much 
myself on many occasions, often removing each lateral growth in one mass.

Freyer has shown that in some cases the whole adenomatous growth can be shelled out in one piece. To accomplish this, the preliminary incision through the mucous membrane into the adventitious capsule that surrounds the enlargement must be shallow, so as not to divide completely the laminæ which form the outer envelope. If the finger hits off a cleft between these outer layers, it can be carried rapidly round the mass, and the whole of the growth can be enucleated in one piece. But if the incision is deeper, and these outer laminæ are divided, secondary layers surrounding only a portion of the growth are exposed, and the enucleation has to be carried out piecemeal.

Whenever portions of any size are removed from the lateral lobes, some of the mucous membrane that lines the prostatic portion of the urethra comes away with them. It can be recognised as a smooth thin membrane closely adherent to the growth. When the whole growth is shelled out in one piece, the prostatic portion of the urethra usually comes away too, and the lining of the membranous part may be torn out with it. As a rule this does not lead to any trouble afterwards, though the attempt should always be made to avoid it by commencing the enucleation near the apex of the gland and working towards the bladder; but I know of one case in which a very obstinate and serious form of stricture resulted.

In those cases in which there is but little adenomatous growth, and the prostate from the first has been hard and fibrous, enucleation will not succeed. No adventitious capsule is ever formed. For such as these either cutting forceps must be used, or recourse must be had to the galvanocautery, which can be guided into position by the finger and applied with excellent effect through an ordinary speculum.

Other plans have been adopted to meet exceptional instances. Thus Tobin (Brit. Med. Fournal, March, I891), removed a projecting median lobe by means of a wire écraseur passed up the urethra, guiding it into position through a supra-pubic opening. F.N. Otis (communicated by IV. K. Otis) on one occasion adopted a similar method, 
passing a wire upwards through a perineal incision. Chismore, of San Francisco, made use of a chain écraseur. And Belfield (Int. Four. of Med. Science, Nov., I 89o) shelled out an obstructing mass of considerable size by pressure from below through an opening in the urethra.

I have never known the hæmorrhage to be serious; the prostate itself (unless it is inflamed or congested) is not a very vascular organ; but in some cases it is reported to have been very profuse. Irrigation with hot water (temperature II $0^{\circ} \mathrm{F}$., so hot as to be unpleasant to the hand) usually controls it without difficulty. If this fails the wound can be plugged with a pad of gauze, soaked in adrenalin. A stout silk ligature should be fastened to the centre of the pad and one end of the silk brought out through the urethra, the other through the supra-pubic wound. The former serves to draw the pad down into the bleeding cavity, the latter to extract it after the bleeding has stopped. In one of my cases in which there was serious recurrent hæmorrhage from some source that could not be found, this answered admirably.

Prostatectomy should never be performed shortly after an attack of retention of urine. Time should be given for the congestion to subside. Not only is the hæmorrhage likely to be more severe, but it is impossible in such circumstances to make certain how much of the obstruction is due to organic overgrowth which will not disappear, and how much to vascular engorgement which will. Much more might be done than is absolutely required.

When there is severe cystitis and the urine is loaded with septic organisms the supra-pubic operation should be divided into two stages. The bladder should be drained first by cystotomy. Afterwards when the urine has become inoffensive and the patient has had time to rest and recover some of his strength, the obstruction at the neck can be removed. It was partly owing to the neglect of this precaution, partly owing to the fact that the operation was performed upon patients who were already in extremis, that the mortality of the earlier cases of supra-pubic prostatectomy was so high.

The amount that must be removed varies with each case. In some, three or four ounces by weight have been removed; in others a fragment only the size of a pea. But whether the 
operation is performed because of intense vesical irritation or because of mechanical obstruction, it is essential that the whole of the additional growth should be removed, if it is to prove a success. A very large proportion of the failures and relapses that have occurred after prostatectomy are due to the simple fact that the removal was inadequate. There can be no doubt that this was the reason why prostatectomy performed accidentally during lithotomy was so rarely successful. A projecting portion of the gland was removed (generally wrenched off by the lithotomy forceps), but not the real obstruction. This is of some importance, because the failure of these cases has often been brought forward to the discredit of this operation.

One of the most common forms of obstructive enlargement, for example, is that in which the sides of the prostate grow upwards, elongating the urethra and compressing it into a narrow slit, until they project into the cavity of the bladder, raising between them a bar which sometimes consists merely of a fold of mucous membrane, but more often contains an actual outgrowth of prostatic tissue. Under these conditions excision of the median obstruction alone is of little or no use. As was recommended by McGill, the projecting portions of the lateral lobes must be removed, and then, taking advantage of the division of the mucous membrane, and the exposure of the surface of the growth, a sufficient amount must be enucleated from the sides to render the passage really funnel-shaped. Surrounded as it is by rigid tissues which have lost all their natural flexibility, nothing else will answer.

Partial removal of the enlarged prostate has, it is true, been followed upon several occasions by a further reduction in the size of the gland, and by apparently a disproportionate amount of improvement in the action of the bladder. The same result is recorded as having followed incision, drainage through the perineum (Harrison), puncture with the galvanocautery (Wishard), and drainage through a supra-pubic opening (Packard and Broome). For this reason it has been suggested that complete enucleation of the obstructing body is unnecessary, and that partial excision will answer; what is left will atrophy and leave the route clear, and the result 
will be equally good, with smaller risk. I have, however, shown elsewhere that there is absolutely no proof that the reduction in size in cases of this kind is ever the result of atrophy. In none does the diminution appear to have been more than could reasonably be accounted for by the subsidence of congestion, cicatrization and fibroid contraction of the adenomatous growth. In nearly every instance in which the amount of improvement was greater than had been anticipated there had been recent acute retention of urine or septic cystitis or both. Under these conditions the prostate always undergoes an enormous but a temporary increase, and the symptoms are made infinitely worse by the swelling of the mucous membrane and the plugging of the periprostatic plexus with thrombi. Such cases often improve materially when treated by catheterisation and rest in bed, the gland diminishing very greatly in size in the course of a few weeks; and, in the absence of all microscopic proof, there is no evidence that the benefit which was undoubtedly experienced in some of these cases was not due to a similar cause.

There is another fact, moreover, which must be taken into consideration before it can be admitted that atrophy of the enlarged prostate can be caused by partial removal. It must be shown that the ejaculatory ducts have escaped injury, a very unlikely result in cases of operation through the perineum or puncture with the galvano-cautery. Section of the vasa deferentia is followed by diminution in size of the enlarged gland in a certain proportion of cases, and there is no inherent improbability in the suggestion that the same result may follow injury to the ejaculatory ducts.

Whether a perineal incision should be made in addition to the supra-pubic opening depends upon the necessity for drainage. It is sometimes of advantage when the urine is particularly foul. The addition of the boutonnière increases but slightly the injury to the tissues and the duration of the anæsthesia, while it drains most effectually the conical cavity which is left after enucleation. But I have rarely found this necessary, and it is questionable whether it would not be better in such circumstances to postpone the enucleation and perform the operation in two stages.

A large soft drainage tube should be secured in the bladder 
through the supra-pubic wound, and the other end conducted to a vessel under the bed. The upper end of the wound should be closed, taking care to bring the aponeurosis together as well as the skin, and some soft absorbent dressings applied. These must be changed every two hours or as often as they become saturated. The patient should be encouraged to sit up in bed as soon as he has come round from the anæsthetic, and should be got up as soon as his strength will permit. The tube can be removed at the end of the second day, as then there is no further risk of urinary extravasation in the prevesical space.

The wound as a rule takes about three weeks to close. In one case under my care in which both lateral growths were removed, it was sound and the whole of the urine was passed per urethram by the seventeenth day.

Supra-pubic prostatectomy is inadmissible in those cases in which the bladder has been reduced by persistent cystitis to a small, hard-walled sac, incapable of holding more than a few ounces. In such the space is so limited that the necessary manipulation cannot be carried out. On the other hand, it is the only method by which vesical outgrowths which have spread from the neck of the bladder along the inner surface of the muscular coat can be excised. And it is by far the best method of dealing with valve-like structures situated at the vesical orifice, and with growths in the lateral lobes.

Through a supra-pubic opening the interior of the whole of the bladder and of the prostatic urethra can be examined, and inspected thoroughly; calculi can be removed; phosphatic debris scraped from the wall; the existence of sacculi ascertained; the exact amount and shape of the enlargement and the relation that it bears to the cavity made out; the degree of resistance in the posterior wall of the urethra felt; the effect which the removal of the obstruction has upon the outlet from the bladder accurately gauged at the time, so. that no more, or no less, than is necessary is done; hæmorrhage can be dealt with more easily than it can through a perineal opening, and unless there is some special condition present, the bladder can be drained equally well.

The rate of mortality after supra-pubic prostatectomy 
depends upon the length of time the septic troubles which so frequently complicate cases of prostatic enlargement have been present, and the stage they have been allowed to reach. If the operation is not performed until the bladder has been ruined by long continued cystitis, and the patient's health and strength are hopelessly broken down by want of rest, prolonged suffering and advanced pyelonephritis the mortality is proportionately high. But then the operation does not deserve the blame. This was the case with the majority of the earlier operations in which the mortality reached nearly or quite 25 per cent. Since then, even when cases are taken indiscriminately, without regard to the experience of the operator, it has fallen to 5 per cent.; and in the hands of individual surgeons, with properly selected cases, it has become much lower.

The singular fact which was soon pointed out that the presence of a calculus in the bladder seemed to have considerable influence in reducing the rate of mortality, admits of a very simple explanation. The suffering caused by the calculus was so great that the operation was performed to relieve the patient at a much earlier date than it would have otherwise been done, before the condition of the kidneys was so desperate as almost to preclude the possibility of recovery.

The measure of recovery depends upon the same causes as the rate of mortality. For supra-pubic prostatectomy to be considered a success it must leave behind it no after trouble, such as fistula; the patient must be able to empty his bladder fairly well, if not completely; and there must be no recurrence of the growth or vesical irritability.

I have not met with any instance in which a supra-pubic fistula has persisted. I have known the scar reopen sometimes in the course of the first year, the union breaking down and allowing a little urine to escape, but I have never known a case that refused to heal as soon as the cystitis was cured. If the exit from the bladder is free, the persistence of a fistulous opening is almost always dependent upon septic inflammation. Closure is the rule unless the wall of the bladder has been so disorganised by cystitis that it is unable to recover.

The same thing is true of the restoration of expulsive 
power. Supra-pubic prostatectomy cannot give back their strength to muscular fibres after they have been destroyed by inflammation, or have been persistently over-strained. A certain amount of residual urine, therefore, is to be expected. But if the obstruction at the exit is thoroughly cleared away the amount of urine left is rarely sufficient to cause anxiety or to require the introduction of a catheter. In a fair proportion of cases, the wall of the bladder regains the whole of its former power and the bladder empties itself completely.

The question of recurrence depends partly upon the nature of the growth, partly upon the freedom with which removal has been effected. It is recorded as having occurred in a few instances, but the proportion is a very small one, and some of these were in all probability examples of malignant disease of the prostate in its early stage, when it is not easy to make a diagnosis. It is not to be expected, however, that a very soft adenomatous prostate would cease to grow simply because a large portion of it had been removed. Enucleation, if the result is to be permanent, must be effectual.

A certain degree of irritability of the bladder occasionally persists after supra-pubic prostatectomy. In some instances this is due to the removal having been incomplete. A portion of the growth has been left at the neck of the bladder; the outlet is uneven and irregular and the symptoms persist. In those cases, however, in which the wall of the bladder has been deeply involved irritability sometimes continues in spite of the obstruction having been removed. The pathological changes induced by prolonged cystitis have grown too deep to disappear at once. But even if micturition is unduly frequent, the intervals are always longer; there is not that instant call, which must be obeyed at once, wherever the patient may be, and there is no need for the habitual use of catheters.

\section{Perineal Prostatectomy.}

A great deal of ingenuity has been expended in devising methods by which an enlarged prostate can be excised through the perineum. In some of these the urethra is 
opened at the apex of the gland, as a preliminary measure, and all the manipulation is carried out through this incision. In others the prostate is approached from its posterior surface, and the urinary passages are preserved intact. I shall deal with the latter first. At first sight they appear to be the more serious of the two; but they possess this great advantage, that the urine, which in cases that have reached this stage is certain to be full of septic organisms, never comes into contact with the wound.

The two most important are von Dittel's and Nicoll's.

Von Dittel's operation consists in removing a wedge-shaped portion from the under surface of each of the lateral lobes. The idea is that the inner surfaces of the enlarged lobes will recede from each other and leave the urethra open. The patient may be placed either in the prone or the lateral lithotomy position. The bladder is emptied, a catheter tied in, and the rectum filled to establish its position and to avoid injury. The external incision is carried from the tip of the coccyx, around the sphincter ani, to the median raphe in front. This opens up the ischio-rectal fossa freely. Then the rectum is separated from the right lobe of the prostate, and the latter exposed. A little further dissection beyond the middle line enables the same to be done upon the other side; and then as soon as the bleeding has ceased, and a good view can be obtained of the parts exposed, a wedge-shaped piece is removed from each. This was proved by demonstration upon the dead subject to be capable of relieving prostatic obstruction when due to increased thickness of the lateral lobes; it allows their urethral surfaces to fall apart from each other, and restores the shape of the canal, which in such cases is practically compressed into a slit.

This operation was devised by von Dittel (Wiener Klin. Woch., 1880-9o; Wiener Med. Woch., November, I89o) who, however, only tried it upon the dead subject. It has never become popular, partly because enucleation, when the enlargement is due to adenomatous growths, is a more satisfactory method of proceeding than excising a formal wedgeshaped portion; partly because, in the majority of instances in which it has been tried, the membranous portion of the urethra or some other important structure has been injured, 
and in several a urinary fistula has been left. In this operation, too, I prefer what is known as Zuckerkandl's incision," through which the posterior surface of the prostate can be exposed with much less injury to the surrounding structures. I have adopted it on several occasions for other operations upon the prostate and vesiculæ seminales, and it gives an excellent view of the gland.

Nicoll's operation (Lancet, April I4, I894) like von Dittel's, aims at keeping the mucous membrane of the bladder intact. The perineal wound is not allowed to communicate with the bladder or urethra. With this object a supra-pubic incision is made as well; and the fingers of one hand are inserted through it into the bladder, so as to force the prostate to some extent down into the perineum, and give warning at once if in the process of enucleation the mucous membrane of the bladder or urethra is approached too closely.

Supra-pubic cystotomy is performed first. The wall of the bladder is secured to the skin by four sutures, the mucous membrane thoroughly cleansed, and the cavity partly filled with carbolic solution (one part in two hundred). The patient is then placed in the lithotomy position and a sound or bougie passed and given to an assistant. "The left forefinger in the rectum, an incision is made in the perineal raphe and gradually deepened until the apex of the prostate is reached, but without penetrating the urethra or the bladder. The rectum is carefully separated from the posterior surface of the prostate, and a vertical incision is then made through the posterior and inferior part of the prostatic capsule. The capsule

* Zuckerkandl's incision (Wiener Med. Press, 21, p. 857, 1889). The patient is in the lithotomy position. An incision, two inches and a half in length, is made transversely across the perineum, a little over an inch in front of the anus. From the two ends of this incision the knife is carried backwards and somewhat inwards towards the middle line for an inch and a quarter. The flap is dissected up until the prostate is exposed. It is then turned back, carrying the rectum and anus with it, and laying bare the trigone, vesiculæ seminales and vasa deferentia. By drawing the rectum backwards the peritoneum is put upon the stretch, and if required can be easily pushed up, so that the whole of the posterior wall of the bladder is open to careful examination. Langenbeck's incision, under the pubic angle, in comparison with this, does not give enough room, and is liable to wound the prostatic plexus. 
is then gradually shed off the gland to either side by means of some blunt instrument like a periosteum elevator, the assistant meantime pressing the prostate down into the perineal wound with his fingers, introduced into the bladder by the supra-pubic opening. (If sufficient room for this isolating of the prostate has not been afforded by the median perineal wound it may be obtained by supplementing the median incision by a lateral one, somewhat on the lines of Dittel's, and curving outwards and backwards from the posterior end of the median cut to a point between the anus and the posterior end of the ischial tuberosity, nearer the former than the latter. This may be done on one or both sides). All bleeding points being secured, the surgeon, after carefully washing his left index finger in carbolic solution, puts two fingers of his left hand into the bladder from the supra-pubic wound, and, pressing the prostate (now pretty freely movable) down into the perineal wound, removes, by the fingers of his right hand in the perineum, the entire prostate, or as much of it as he deems necessary to relieve the neck of the bladder from all pressure, and bring it down to a level with the post-prostatic pouch in the base of the bladder, judging the amount to be removed by manipulation of the parts between the fingers of the two hands. Should the prostatic tissue be more than usually tough and resistant, the fingers of the right hand may be supplemented by a blunt periosteum elevator or other similar instrument, or even by a Volkmann's spoon. carefully used. During the entire operation neither bladder nor urethra is opened further than is involved in the supra-pubic incision. After enucleation of the prostate is complete the sound or bougie is withdrawn and a large-eyed short-beaked metal catheter (such as a lithotrity evacuating catheter), or a large elastic-gum catheter coudé is introduced and tied in. The perineal wound, which forms a cavity of some magnitude, is douched with weak carbolic solution, carefully dried with gauze or sponges, and firmly stuffed with iodoform gauze. The four stitches in the supra-pubic wound are cut and the bladder wall thus permitted to drop away from the parietes. The after-treatment consists mainly in douching out the bladder daily with boracic or weak carbolic solution, from the supra-pubic wound into the bladder, and out through. 
the catheter in the urethra. The iodoform packing is changed every second or third day for a fortnight and then withdrawn, and the wound allowed to close. The supra-pubic wound, which is kept closed by a pad of sublimated gamgee, also begins to close about this time, when the douching may be discontinued.

"The advantages this method of prostatectomy appears to offer in comparison with the others are:-(a) There is much less hæmorrhage. This is but what might, à priori, be expected. The prostatic plexus of veins lies on the surface of the prostate (not in its substance, which is little vascular) and is consequently largely shed off with the capsule by the blunt periosteum elevator. Further, the plexus clothes mostly the anterior and lateral aspects of the organ. In approaching the prostate on the posterior and inferior surface few veins are encountered. (b) The infiltration of the freshly wounded tissues by the putrid and septic urine is avoided. In the removal of the body and lateral lobes on any adequate scale a large gaping cavity is created. In the supra-pubic operation no means of efficiently draining this exists, nor is the perineal tube introduced in the perineal or combined operation of any service in the matter, the greater part of the cavity lying below the level of the aqueduct from the bladder to the skin orifice along the tube, and thus retaining the fluid which gravitates into it. By the operation described this cavity is kept absolutely free from either putrid or putrescible urine and filled with a dry antiseptic medium. (c) The operation insures adequate and sufficient removal of the obstructing prostatic tissue, thereby avoiding one main source of ultimate failure to give relief to the symptoms in both the perineal and supra-pubic operations when uncombined. (d) By preserving the bladder wall intact there is very much less risk of tearing out portions of the deep urethra and bladder wall, with the subsequent possible results mentioned above. With a bougie in the urethra and two fingers in the bladder there can with due care be very little chance of removing any of the bladder or urethral wall with the prostatic tissue. Such tearing out of portions, not unknown in the supra-pubic operation, is comparatively common in both the perineal and ordinary combined perineal and supra- 
pubic operations. (e) The absence of a perineal tube permits. the patient to sit up without inconvenience from the first."

A similar operation has been performed by Macewen.

Alexander's operation closely resembles Nicoll's, but differs in that the urethra is opened upon a grooved staff and a perineal tube tied into the bladder after the operation has been completed. The bladder can be drained in this way through. the perineum without infecting the wound. Parker Syms has suggested that laparotomy should be performed instead of median cystotomy, and that the hand should be introduced into the abdomen instead of into the bladder, for the purpose of depressing the prostate and bringing it within reach of the finger in the perineum. And Alexander has achieved the same result in a thin subject by pressure upon the abdominal wall above the pubes, without opening either bladder or abdomen. But it is to be noted that in both these cases the guiding sensation of the operator's fingers pressing upon the vesical surface of the prostate is lost. Proust exposes the posterior surface of the prostate through an incision similar to that devised by Zuckerkandl, separates it from its attachments, and then incises the prostatic urethra along its floor for its whole length. Each lateral half of the enlarged gland can then be cut away without difficulty.

Nicoll's or Alexander's operation is capable of a rather wider application than von Dittel's, and enables enucleation to be carried out with greater accuracy, but all these operations have the same drawbacks. Vesical outgrowths (and they constitute the majority of cases which require prostatectomy) must be approached through the bladder. Nicoll himself recommends that if there is a median vesical projection, it should be left at the time of the operation, and removed later in this way, after eight or ten days, when the urine has become aseptic and the perineal wound is covered over with granulations.

The other form of perineal prostatectomy, that in which an opening is made into the urethra at the apex of the prostate and the manipulation is carried out from the inner surface of the prostatic urethra, is limited to cases of purely median urethral or orificial growths, and those in which drainage of the bladder is the main object, and the treatment of the enlargement is secondary. 
The distance of the vesical orifice from the surface of the body-the perineal distance as Watson has called it-precludes anything more than this in a large proportion of cases. If it is more than three inches the finger cannot reach the bladder, much less explore the interior around the outlet. It depends partly upon the condition of the perineum, whether it is rigid or loaded with fat, partly upon the extent to which the lateral lobes have grown up and carried the bladder before them. So long as the perineum is soft and yielding the prostate and the neck of the bladder possess a considerable range of mobility during life; they can often be pulled down within reach, and quite half an inch gained. In several cases in which I have operated by this method I was very much struck by this. It was not possible at first to enter the bladder, but by passing a short, sharply curved sound by the side of the finger, and hooking it against the trigone, the whole of the ring could be brought down and examined with ease.* Unhappily, in the majority of cases of enlarged prostate, the rigidity of the tissues makes this impossible; and of course the lengthening that is due to overgrowth of the lateral lobes is unalterable.

Watson, who regards the perineal operation as the safer, and the method of choice when it can be done, in his admirable monograph on the "Operative Treatment of the Hypertrophied Prostate," states that although his finger is of full average length, he must have failed to reach the bladder in at least one-third of the thirty cases which he has figured, and which he examined with this object in view. With regard to this, however-although I believe his conclusions are in the main correct, I would venture to point out that the specimens upon which they were founded were taken from a museum, in which, presumably, they had been preserved because of the way in which they illustrated the final effects produced by enlargement of the prostate, and without any reference to their clinical history. It does not follow that

* Parker Syms (Brit. Med. Four., Nov. 8, I902) employs for this purpose a rubber retractor with a hollow bulb at one end. This is introduced into the bladder empty, and then distended with water by means of a piston syringe, so that when the retractor is pulled upon, the shoulder of the bulb catches against the vesical surface of the gland. 
operation was required or would have been advisable in all or any of these. It is not the mere fact of overgrowth, however great this may be, which renders operation justifiable, but the obstruction and the irritability caused by it ; and no conclusion, such as that drawn by Watson, is warranted until the perineal distance has been measured in a series of cases in which operation by one route or the other is considered advisable. For this reason, McGill's observation, that in only three out of his first twelve cases would it have been practicable to remove satisfactorily the projecting portion of the prostate by the perineal route, is of more value, and probably gives the true proportion.

Another great objection against this method is the very small amount of space that it allows for the necessary manipulation. A small pedunculated median lobe can be removed, or a V-shaped channel can be punched out or burnt out from the posterior margin of the vesical orifice; but this is the limit. Nothing can be done for the lateral lobes if they are enlarged. Vesical outgrowths, which, as Fergusson long since pointed out, are often the most serious from the intense irritation they cause, almost as bad as that attending phosphatic calculi, are quite beyond reacl. A median lobe of any size, even if it is pedunculated, can only be extracted through the narrow outlet in fragments, and as the urethra in such cases is usually compressed into a cleft by the growth of the lateral masses, this entails such an amount of manipulation and bruising that the rest of the gland is very likely to slough. The method is plainly only suited to a very limited class of cases, and those not the worst, or requiring operation the most.

On the other hand where the main object is drainage of the bladder and subsidence of congestion, perineal prostatomy (that is to say, simple division of the obstructing portion in the middle line) if followed up by the prolonged retention of a drainage tube, compares very favourably as a temporary measure with the other methods of drainage already described.

Division of the prostate in the middle line was first suggested by Guthrie. A grooved staff is passed down the urethra; the membranous portion laid open; and the finger 
introduced into the prostatic part. The obstruction is divided with a probe-pointed knife in the median posterior line, and the division completed, either with a staff or with the finger, according to the degree of resistance found. There is little or no hæmorrhage, and so long as the patient is confined to bed one of Annandale's drainage tubes can be worn with comfort. After a period varying from six to twelve weeks it is found either that the urine passes down the urethra voluntarily, or that a catheter can be passed without difficulty, owing to the way in which the prostate has contracted. The tube is then withdrawn, the opening allowed to close, and a bougie passed occasionally.

Perineal prostatomy, performed in this way, without being supplemented by further measures such as drainage, is of very little use. The few cases in which it has succeeded probably owe the benefit they have derived almost entirely to the congestion of the gland having subsided. Nor could it well be otherwise. As soon as the neck of the bladder has recovered from its temporary distension, its natural elasticity and the action of the muscular fibres which surround it bring the torn or divided surfaces together again, and though a small amount of the growth may be lost by suppuration and sloughing, it is not enough to effect any permanent change for the better. The obstruction soon returns.

The prolonged retention of a drainage tube adds materially to the prospect of success. Various instruments have been devised for the purpose and Watson's ("Operative Treatment of the Hypertrophied Prostate") may be taken as an example of one of the best. Its end occupies the lowest part of the bladder, and the eye is so placed that there is no cul-de-sac beyond. Its calibre is of full size; the shaft corresponds in direction with the posterior part of the urethra into which it fits, while its external portion is parallel with the bed when the patient is lying upon his back, and is fastened by tapes attached to a plate, which is so arranged that while it can be pushed backwards and forwards upon the tube to accommodate itself to the difference in depth of the perineum, it will remain at any point at which it is placed. How a tube of this kind acts when retained for many weeks is a matter of opinion; it cannot be simply mechanical dilatation; probably 
the more or less friable tissue of which the prostate is composed undergoes at first a certain degree of disintegration, and then slowly heals over so as to leave a canal of greater width; but it certainly does increase the chance of success. Six out of the eleven cases published in the statistics appended to my "Hunterian Lectures" in which there is mention of drainage for any length of time (and it must be for some weeks to be of any service, though the patient is not necessarily confined to bed), were returned as cured, and two as much improved. Two, however, were distinct failures; a third was very little benefited, and in one of the successful ones a small fistula persisted.

So far as enlargement of the prostate is concerned perineal prostatomy through the urethra cannot be considered in any way a radical cure, except in those rare instances in which the whole of the obstruction depends upon a valvular median lobe; but where only the least possible can be done, where the bladder is either in a state of atony or else rigid and contracted, where the patient is worn out by septic absorption and prolonged want of rest, I have known median division followed by drainage prove of great service and enable catheterisation to be resumed after a time with a fairly long interval between each introduction.

The galvano-cautery has been applied to growths around the neck of the bladder through the prostatic urethra by Wishard (Foumal of Cutaneous and Genito-Urinary Diseases, March, I892) on several occasions. A cylindrical rectal speculum, with an aperture three-quarters of an inch in diameter, was passed first through a perineal incision in order to protect the mucous surface. And Watson (loc. cit.) has devised a galvano-cautery prostatome which may be used either through a perineal wound or after supra-pubic cystotomy. "It has the form of a short, broad-bladed lithotrite, each blade being reduced to a thin edge by a large central fenestrum of oval shape. Each blade bears a rim of petrified wood for the purpose of isolating the loop of platinum wire which rests upon the surface of the inner blade, after emerging from the hollow inner shaft, through which the wires are conducted to the battery connections. The obstructing portion of the prostate may be grasped be- 
tween the two blades, and its central portion removed, or the whole taken away piecemeal as its form may dictate, by pressing the blades together, the wire being heated by the electric current." Bottini's instrument may be used in the same way.

Many of the disadvantages which attend the urethral methods of dealing with enlargement of the prostate, can be avoided by making a preliminary opening in this way through the perineum. The size and shape of the obstruction, for example, can be made out with the finger. The bladder can be drained, and any hæmorrhage that occurs either during the operation or later, when the sloughs are beginning to separate, can be dealt with more easily. The operation may be a little more severe than the urethral, and the patient may have to keep in bed a few days longer, but it is certainly more efficient. It is not, however, of the least use if the growth has attained even a moderate size.

Many other devices have been tried to render this mode of operating more successful. Gouley and Norton have planned instruments with cutting blades somewhat after the fashion of a lithotrite, to cut out or punch out portions of the gland. On one occasion I used a wire snare, guiding it round the neck of a valvular upgrowth by means of the finger in the urethra. In another case I twisted off a median upgrowth by means of polypus forceps. But methods such as these are plainly only suitable to special cases.

\section{Urethral Operations.}

The only method of operating through the urethra which requires mention is Bottini's. This consists in burning a channel through the obstruction at the neck of the bladder by means of the galvano-cautery applied through the urethra. There is no external incision.

Bottini's instrument, as modified by Freudenberg, resembles a lithotrite in shape, with a male blade, made of platinum-iridium, flattened from side to side until it resembles a knife edge, and fitting into a deep recess in the female one. The handle of the instrument is hollow, and 
arranged so that a current of cold water can be kept circulating through it while the male blade is being raised to a white heat.

No anæsthetic is required, if, that is to say, the urethra is fairiy tolerant of instruments. The bladder may be distended with water, but this is not absolutely necessary. Of late I have made use of air instead, and it seems to me to answer the purpose better of holding the rest of the wall of the bladder out of the way of the cautery. When the point is in the bladder it is turned downwards as in searching for a stone, and gently drawn outwards until it is felt that the beak is in contact with the obstruction. If there is any doubt, the exact position of the instrument should be ascertained with the finger in the rectum. The current is then turned on and the curve pressed against the hypertrophied tissue, a stream of water being made to circulate through the instrument so as to prevent the shaft becoming too hot. By gently elevating the handle the platinum can be made to burn its way slowly through, a hissing sound being distinctly heard. Then the end of the instrument is pushed into the bladder and allowed to cool down. If the scab sticks to it, it must be burnt off. I usually make three linear incisions in this way through the congested mucous nembrane at the neck of the bladder, one in the middle line and one on each side.

The current required to work effectually must be of considerable strength, twenty to thirty ampères. Bottini makes use of accumulators, but if the patient's house is supplied with the electric light, and the current is an alternating one, the required amount can be more easily obtained by means of a Woakes' transformer.

This method of treating enlargement of the prostate has not become so popular as it deserves to be, owing no doubt to the objection that the operation is performed without any definite knowledge other than can be acquired by urethral exploration, of the size, shape, and position of the obstruction. Bottini, who performed it over one hundred times, with only two deaths (and those among the earliest cases) spoke of it in the highest terms. Freudenberg, who has used it with great advantage (Arch. f. Klin. Chir., B. lxi., 
p. 94I), collected reports of 683 patients operated upon by this method, and estimates the mortality at something over five per cent., or if deaths not directly attributable to the operation are omitted, at a little more than four per cent. In only six per cent. of the cases was the operation pronounced a failure, while in a third of the remainder it was stated there was an improvement, and in two-thirds there was a cure.

The chief risk of the operation is hæmorrhage, either at the time or subsequently when the sloughs are beginning to separate; but this can usually be avoided by making the incisions exceedingly slowly, and by using the blade at a white heat. Temporary retention of urine arising from acute prostatitis, and frequent and painful spasms at the neck of the bladder are also stated to have occurred; and the bladder wall has been perforated, but it is difficult to understand how this could happen if the bladder itself is well distended with air, and the finger is introduced into the rectum. The operation does not necessitate prolonged confinement to bed; and it is not followed by psychical disturbance in the same way that orchidectomy may be. If it fails, it leaves no bad results, such as fistula, and no mutilation.

It is of no use in enlargement of the lateral lobes; nor when the growth has spread into the bladder and become vesical (a form which frequently gives rise to the most intense irritation, so that the patient is often sounded time after time for calculus); nor if the growth has attained any size. The only cases in which it is of real service so far as adenomatous overgrowths are concerned, are those in which there is a small sessile obstruction in the posterior wall at the vesical orifice. This can sometimes be removed with a fair degree of certainty and with practical safety.

There can be no doubt from the great success which this method of operating has attained in competent hands, that the benefit derived from it cannot be due simply to the removal of a passive obstruction. It must have some other action, probably upon the congested mucous membrane. In many cases the earlier symptoms caused by prostatic enlargement (and indeed those that occur throughout the whole course of the disease, more or less) are due almost entirely to congestion of the mucous membrane at the neck of the 
bladder. This is the cause of the irritability of the bladder, of the increased frequency of micturition, of the obstruction at the outlet (to some extent), of the residual urine in part, and later, when it becomes chronic, of the degeneration of the wall of the bladder. A vicious circle is established; the congestion makes the bladder irritable, and the constant straining makes the congestion worse. Bottini's operation breaks this circle effectually, and almost without risk. The cautery, cutting into the mucous and submucous layers, destroys the venous plexuses, and the linear cicatrix that it leaves finishes the work. It does not stop the enlargement of the prostate, and it has no effect upon extensive outgrowth, but it does prevent many of the distressing symptoms to which the enlargement gives rise, and by stopping the congestion it can, at least, delay the consequences that follow. Many of the cases in which I have used it have derived material benefit from it, and have been relieved for some years from distressing irritability of the bladder and excessive frequency of micturition without incurring more than a minimum degree of risk. Willy Meyer (Med. Record, April 28, I901) recommends that the operation should be performed as soon as it is necessary to give the catheter into the hands of the patient himself. Certainly if this is done, habitual catheterisation can often be postponed for years.

This method may also be employed through the perineum, as recommended by Watson (loc. cit.). The incision necessary is exceedingly small, but it enables the finger to be introduced, and the condition of the prostate and the neck of the bladder to be ascertained with accuracy, thereby removing one of the great drawbacks to Bottini's method without sensibly increasing the danger. 


\section{CHAPTER XI.}

The Effect ufon Enlargement of the Prostate of Orchidectomy and of Operations upon the StructURes contained in the Spermatic Cord.

In the previous chapter I stated that there were two different ways of dealing with the obstruction at the neck of the: bladder caused by enlargement of the prostate. One is partial excision, prostatectomy. This I have described already. The other is the induction of atrophy by means either of orchidectomy, or of an operation performed upon some of the structures contained in the spermatic cord.

Before considering the value of this proceeding there are two points that deserve attention. One is the fact that the increase in the size of the gland is not pure and simple overgrowth. Congestion is a very important factor. I am probably well within the mark in saying that in many of the cases under my care, one-third of the increase in the size of the gland at the time that the patients consulted me was due to this cause alone. When there have been repeated attacks of inflammation of the bladder, lasting perhaps for months, and leaving behind an enormous amount of swelling and induration of the mucous and submucous layers which surround the neck of the bladder, this addition is even more important.

The other point to which I wish to call attention is that many of the worst and most distressing symptoms which occur in connection with enlargement of the prostate are the direct result of this congestion and chronic inflammation far more than they are of the actual glandular overgrowth. I readily admit that without this overgrowth there would be no congestion or inflammation, or so little and so transient that the effect would not be material. But this does not alter the fact that the vascular and nervous changes are to a great extent the direct and immediate agents.

It is evident, therefore, that so far as the relief of symptoms 
is concerned, the congestion and chronic inflammation of the gland and mucous membrane lining the neck of the bladder are of scarcely less importance than the glandular overgrowth itself.

The influence which the testes possess over the size and growth of the normal prostate has been known for many years. Long ago Hunter proved that removal of the testes in young animals prevents the development of the prostate: that a similar operation in adult ones causes the fully developed gland to atrophy and waste away: and that in those animals in which the development of the sexual organs varies with the time of year the prostate presents differences in size and structure quite as striking as those presented by the testes. And these conclusions have been confirmed by numbers of observers since.

That the first two conclusions are equally true of man is also a matter of ancient history. The number of observations is smaller but they are all positive, none even doubtful. It is to be feared that the significance of these experiments and observations was not appreciated as fully as it should have been. In spite of this definite evidence connecting the prostate with the sexual system, anatomists and surgeons continued for a long time to regard the gland as an appendage to the bladder and in some way or other concerned with micturition. Not until it was shown beyond question that the prostate was strictly and entirely a sexual organ and nothing else was it possible to make any further advance. At the present day it is difficult to understand how any other view could ever have been maintained.

The next step, curiously enough, arose from what is now admitted to have been a mistake. It was imagined that the prostate, because in the course of its growth it had included and enveloped the utricle, was homologous with the uterus; and that therefore the development of prostatic glandular overgrowth was homologous with the formation of uterine fibro-myomata. It was suggested that, since oophorectomy under certain circumstances was followed by shrinkage of fibro-myomata, orchidectomy might be followed by atrophy of the enlarged prostate. The premises were entirely wrong; the prostate is not homologous with the uterus; its homologue 
lies in the perineum; and of course the pathological changes which take place in the one are in no wise homologous with those which take place in the other; but the conclusion turned out to be correct; the relation that exists between the prostate and the testes does admit of comparison with that which exists between the uterus and the ovaries.

Influenced by these arguments and by the results of experiments upon dogs, Ramm of Christiania, in April, I893, operated upon two men, seventy-three and sixty-seven years of age respectively (Centralblatt $f$. Chirurgie, No. 35, Sept. 2, I893, and No. I7, April 28, 1894) with an excellent result. In each case there was rapid improvement, the prostate steadily diminishing in size until it could only be felt as a small flat mass, and the bladder regaining its power so that the patients had only to rise, one of them once and the other twice in the course of the night to pass water.

In June, I893, White of Philadelphia, first collected and published with unmistakable clearness the evidence clinical, experimental and pathological, which would justify a resort to this operation. Acting upon White's suggestion, Haynes of Los Angeles, California, performed orchidectomy with the view of inducing atrophy of the enlarged prostate on three occasions in December of the same year.*

Since that time orchidectomy has been performed in a large number of cases. Unfortunately in most of those that have been published the details are given in such an unsatisfactory manner that it is impossible to draw from them any

* As frequently happens in such cases the same idea appears to have occurred more or less definitely to many people almost at the same time. According to Walker (New York Med. Fournal, April, 1895) Tupper performed orchidectomy with the definite object of giving relief from prostatic troubles on two occasions in 1882 and 1886 , after having seen the effect produced by removing the remaining testicle from a patient who had already lost one by accident. Launois suggested it in 1884 to Guyon. In November, 1892 , shortly after the publication of my Hunterian Lectures, I discussed the advisability of it with a patient, who, though he admitted the force of the arguments in favour of it, not unnaturally declined it on the ground that it must be of the nature of an experiment.

I am informed by a friend that the practice is well known among veterinary surgeons, who are in the habit of performing castration to relieve retention of urine in the case of old dogs suffering from enlargement of the prostate. 
conclusion that is worth having. Some of the cases, if the lists are carefully examined, were clearly unsuitable. In some there was malignant disease of the prostate. In others there was no evidence of anything but constantly repeated attacks of chronic inflammation. In many instances the result was published much too soon, often within a few days of the operation, instead of waiting for an interval of at least six months. And in others again no allowance was made for the presence of calculus or of recent retention of urine or of other methods of treatment that were adopted at the same time. I shall therefore, so far as my conclusions are concerned, rely almost entirely upon my own cases, or upon those which I have seen in consultation with others.

\section{The Effect of Orchidectomy upon EnLargement of} the Prostate.

Diminution in the size of the prostate followed in every one of my cases.

In some the diminution in size began within a few hours. Patients who were suffering from acute retention, and some even of those who had been unable to pass urine without a catheter for years, were in several instances able to pass a small quantity voluntarily, without assistance of any kind, on the evening of the operation. In some the improvement continued until the normal power of micturition was restored. In others it disappeared again in the course of two or three days and the power of voluntary micturition only returned after the lapse of some weeks. In two of these cases there was for some days a marked degree of muscular spasm affecting the compressor urethræ and the sphincter ani.

The diminution in size was estimated partly by rectal examination, partly by the difference in the distance it was necessary to pass the catheter, before and after the operation, in order to draw off the urine. In the case of one patient, an enormously stout man, who died from fatty degeneration of the heart nine days after the operation, it was evident from the wrinkled condition of the mucous membrane and the 
softened and shrunken state of the vesical outgrowths that a considerable alteration in size had taken place.

These results of mine agree fairly well with those that have been collected from the medical journals. According to these statistics diminution in size of the enlarged prostate takes place in from 82 per cent. to 89 per cent. after double orchidectomy. Making allowance for the fact that, as I have mentioned already, some of these cases were unsuitable, not being cases of true enlargement, and that others were published long before the final result was known, the difference between these statistics and my own results is probably a good deal less than it appears to be. In any case I attach very little importance to statistics of this kind, collected together indiscriminately from different countries and all sorts of sources. The results can only be approximate. The data are not given at sufficient length or with sufficient uniformity to enable any exact comparison to be made or any binding conclusion drawn.

I have not been able to trace any connection between the diminution in size of the enlarged gland that occurred in my cases and the presence or absence of living spermatozoa. Their existence does not appear to be material. Nor does it appear to make any difference whether one of the testes has been rendered useless by epididymitis in early youth. I have not had under my care a case of enlargement of the prostate requiring operative treatment in which both testicles were small and shrunken from this cause. Whether bilateral epididymitis would have any influence in preventing the occurrence of enlargement I cannot say.

There cannot be the least doubt that the diminution in size of the enlarged gland and the relief which are so often noted after orchidectomy in the course of the first few days, and sometimes in the course of the first few hours, are due solely to vascular changes and not to atrophy.

One case upon which I operated recently showed this in a remarkable manner. The testes were removed separately, the second about six weeks after the first. The prostate was very large, the median as well as lateral lobes being involved. There had been complete retention for many years with occasional attacks of hæmaturia and intense dysuria. Within 
a few hours of the removal of the first testicle all the symptoms underwent conspicuous relief. A little urine was passed spontaneously; the pain ceased, and when a catheter was passed the urine began to flow through it while the eye was still in the prostatic portion of the urethra. It is not unusual for this to occur when a catheter is being withdrawn from the bladder, after this has been partially emptied; but it had never occurred before to this patient who noted the distinction at once. Within that short space of time the prostatic portion of the urethra had become so much wider and larger that it was able to hold a certain quantity of urine and was in open communication with the bladder. Three days afterwards all the improvement was lost and no benefit was experienced until the second testicle was removed six weeks later.

It is impossible to imagine that the prostate could have wasted in the course of a few hours and then have grown up again. Atrophy requires time, especially in the aged. The symptoms which compel patients to apply for relief-the dysuria, nocturnal irritability of the bladder and retention of urine are dependent to a very large extent upon the hyperæmic state of the prostate and the mucous membrane at the neck of the bladder. The operation, partly by the shock that follows it, partly by the way in which it cuts off any further stimulus from the testes, checks this hyperæmia, and gives a certain measure of immediate relief.

Cases in which there has been recent acute retention of urine may expect this immediate relief. It is probable that in these, as I have mentioned already, as much as one-third of the increase in the size of the prostate is due to vascular congestion. The relief is not so common or so definite when the operation is performed because of the habitual presence of a large amount of residual urine, or in cases in which there has been constant retention for any length of time. It may not show itself, under these conditions, until some days or even weeks have elapsed.

As this improvement occurs, and is equally well marked, in patients who have been resting in bed and who have already. been kept under the ordinary treatment for enlargement of the prostate for days, and in some cases for weeks, and as it 
occurs immediately after the operation, it cannot reasonably be assigned to any other cause than the operation.

Atrophy of the prostate, to a greater or less extent, has taken place in every case of orchidectomy under my care, with two exceptions. The evidence I admit is only clinical. But except in two instances the diminution in size was so conspicuous that it was impossible to account for it merely by the cessation of vascular congestion. The gland which had been very much larger than normal, in many instances twice and even three times the normal size, shrunk until it could only be felt as a soft flat mass with ill-defined edges, lying behind the urethra. The projection into the rectum that is always present with a normal prostate simply disappeared. In the two exceptions, the gland six months after the operation was still larger than normal, so that, although the size was certainly diminished, it is doubtful whether true atrophy had set in.

The time at which atrophy (not mere diminution in size) begins to be evident, seems to vary within very wide limits. The earliest date recorded is eighteen days (Griffiths, Brit. Med. Fournal, March 16, 1895). In this case, in addition to other changes of a more uncertain character, it is noted that there was acute fatty degeneration with disintegration of the cells which had accumulated in the lumen of the tubules. In White's case which died on the second day after the operation, the microscopic changes described, as pointed out by Albarran (Mal. des Org. Genito-Urinaives, Jan., I898, p. 27) do not in the least justify the conclusion drawn. There was no microscopic evidence of atroplyy (in spite of a considerable diminution in size) in my case although nine days had elapsed. Nor was there any in Kelsey's (Med. Record, 1896) at the end of thirty-eight days; or in Cabot's (five months), or in Socin's (Correspondenz-Blatt fïr Schweizer Aertze, 1896, No. I7) in which the period after the operation was longer still.

In one very exceptional case (Bryson, Boston Medical and Surgical Four., 1896, p. 474) there was no evidence of atrophy even at the end of twelve months. Six days after the operation there was a little improvement. Nine months later retention of urine occurred again. A year after the operation 
no change could be detected in the size or consistence of the gland when it was examined per rectum. There was a calculus in the bladder, to remove which cystotomy was performed. Part of the vesical portion of the growth was excised at the same time and examined under the microscope, but there was no evidence of atrophy.

A good many of the cases in which atrophy is said not to have taken place have been reported much too soon. Others can be accounted for without much difficulty. In one, for instance, there was a large fibromyoma which was shelled out with an excellent result by supra-pubic prostatectomy a year after orchidectomy had been performed. This was probably a genuine tumour and as such of independent growth, not likely therefore to be influenced by orchidectomy, and the same may have been true of Bryson's case. In several the symptoms pointed to long continued chronic inflammation, and the increase in the size of the gland was at least as much due to this as to true enlargement. In others again in which the gland is described as being of moderate size, but very hard, it is probable that the enlarged prostate had already undergone fibroid degeneration, and that there was no gland tissue or stroma left, nothing but an inert fibrous mass. Bryson's case is so far as I am aware the only one in which there is microscopic proof that some degree of atrophy had not taken place at the end of a twelvemonth.

It has been said that occasionally the normal prostate does not atrophy after castration. (Faulds, Brit. Medical Fournal, May, 4, 1895). But it is not said that any microscopic examination was made in these cases, and in the absence of this I cannot accept the evidence in regard to the failure of atrophy as satisfactory. The prostate, for example, in a bullock is often as large as it is in a bull, and might very easily be said not to be atrophied; but the microscopic appearances are entirely different.

There is no means of telling beforehand whether atrophy will be slow or rapid. From the analogy of what happens to the normal prostate after castration, and from the microscopic evidence of such cases as Griffiths', it seems probable that the glandular epithelium is affected first and the stroma later. But it is not known if or when the fibrous tissue, which forms 
so large a part of many enlarged glands, begins to waste. Large growths, provided they are not made up of independent tumours, which are not likely to be affected, diminish in size more rapidly than small ones, and are the most suitable for this operation. I have not found that consistence, apart from size, is of any value as a guide. It is very difficult to form an estimate as to hardness through the wall of the rectum, and unless the examination is repeated again and again it is impossible to say whether a large or a small proportion of the induration is due to congestion and thrombosis. I feel convinced, however, that comparatively small hard prostates accompanied by conspicuous atony of the bladder and a persistently large amount of residual urine diminish proportionately less than any others.

Apart from the question of the diminution in size the degree of relief which follows the operation has been satisfactory in nearly all my cases. The frequency of micturition has diminished. In a large proportion of cases it has disappeared altogether, so that the patient has only to rise once or at most twice, in the course of the night. The dysuria has ceased entirely. There is no longer the constant burning pain at the neck of the bladder that is so distressing; the patient is not compelled to have recourse to the catheter at an instant's notice wherever he may be; and there is no risk of acute retention.

When the symptoms persist it nearly always means that there is some other cause at work as well, such as sacculation, in which case it is almost impossible to free the bladder from septic organisms, or latent calculus. The number of cases in which calculi are present in association with enlargement of the prostate is much larger than is usually believed. For the most part they lie behind the prostate in the post-prostatic pouch, unknown and often unsuspected. Owing to the fact that they are almost fixed, and that they never come into contact with the neck of the bladder, they do not give rise to the ordinary symptoms. Micturition can hardly be more frequent than it is already. There is no pain at the end of the penis, or sudden stoppage in the stream of urine. Hæmaturia if present may be due to the enlargement. But when the prostate diminishes in size the stone becomes free, and 
all these symptoms set in at once, often with great intensity owing to the tender state of the bladder. Needless to say, whether orchidectomy is performed or not, the symptoms due to cystitis never disappear so long as a calculus is present, or there is a diverticulum full of septic urine, which is never emptied.

How far the bladder regains the power of emptying itself depends partly upon the diminution in size of the prostate, and partly upon the extent to which the muscular fibres have been injured already. In the majority of my cases recovery was fairly complete. The removal of the resistance in front enabled the muscular coat of the bladder, with the assistance of the abdominal muscles, to empty the cavity to within a few drachms. The stream was not a strong one, but the result was sufficiently good. In two of my cases only has there been little or no change for the better, so far as this was concerned. They were both cases in which the prostate was not very large and in which there had been repeated attacks of cystitis. But even in these the difference in comfort that is experienced when a catheter is passed admits of no mistake. There is no longer any congestion at the neck of the bladder. Instead of the catheter being required every hour, and sometimes during the attacks of cystitis, almost every half-hour, and instead of the introduction being a matter sometimes of great difficulty and always attended with very severe pain, sometimes with hæmorrhage, the catheter now is not required more than four or five times in the twenty-four hours; it passes in perfectly easily, and there is no pain and no bleeding. The hyperæmia at the neck of the bladder has ceased; and though septic organisms are always getting in, they can do little or no harm, because the bladder is kept empty and there is no retained urine or congested mucous membrane to give them the foothold that they require.

Two patients of mine out of nineteen died, the second and the fourth. One of these died, as already mentioned, from fatty degeneration of the heart on the ninth day; the other from plugging of the left middle cerebral artery on the fourth. In each of these cases the shock of the operation may have been an accessory cause. No mental trouble or weakness has followed in any case under my care; and as might be 
expected, although the secondary sexual characteristics are dependent for their development upon the testes, no alteration of any kind, no change of voice or of appearance has ever taken place.

If all the published cases in which orchidectomy has been performed for enlargement of the prostate are collected together, the rate of mortality following the operation appears to be as high as fifteen per cent. This is very much higher than that which follows the same operation performed in adult life for such causes as tuberculous disease. Calculations, however, of this kind involve so many fallacies that the outcome is of little or no value. Cases in which the patient has been at death's door from suppurative pyelonephritis, and those in which the operation has been performed almost as a forlorn hope, are mixed up with others in which the object in view has been carêfully thought out, and the most favourable time selected. Deaths which have occurred after the operation are placed in the same category as those which have been caused by it. No allowance has been made for the heavy death rate which normally attends such diseases at an advanced time of life. Cases in which no precautions against septic disorders have been taken are counted with the rest. And it is assumed that all operators are equal in experience.

The mortality due to orchidectomy in adult life is scarcely more than one or two per cent. In old age, when the action of the heart is more feeble and the arteries are atheromatous (I have shown already that there is no causal relation between enlargement of the prostate and arterial degeneration, but of their frequent association there can be no doubt) the risk is naturally somewhat greater. But if cases are properly selected and orchidectomy is performed, not as a last chance, but at the most favourable moment, as an intelligent and timely precaution against an inevitable future, there is no reason to think that the rate of mortality would be materially higher.

Apart from causes of death such as sepsis, which can be and should be avoided, and uræmia from advanced pyelonephritis, the chief danger appears to be a form of shock such as is not unfrequently met with after operations and accidents in old people. It is not immediate, nor is it to be attributed 
specially to ligature or section of the spermatic cords. I have often asked anæsthetists to notice the condition of the pulse at the moment that the vas was severed, and contrary to what is usually stated, there is never any difference to be detected. The symptoms come on slowly and insidiously after some hours, even after some days, and they are almost entirely mental in character. I have never myself met with any case in which there was more than a certain degree of nocturnal delirium, such as frequently occurs in old people if there is the least febrile disturbance. But cases have been recorded in which the delirium was alarming, and some in which a condition of childishness and even of acute mania has supervened. It is this, even more than the actual rate of mortality, which has led to orchidectomy being regarded with such disfavour.

That these symptoms are not in any way dependent upon a specific or internal secretion of the testes, which is lost to the organism when they are removed, is certain. They never occur when a similar operation is performed in younger life, when it may reasonably be assumed that the testes are of much higher functional significance than they are in old age. And they have been known to follow the removal of one testis although the other was sound, and even ligature of the vasa deferentia under cocaine. It is true that the administration of orchitic extract is said to have been followed by improvement in one or two instances, but this can hardly be accepted as evidence as to cause.

Unfortunately most of the cases in which these symptoms have followed have been recorded so briefly that it is difficult to draw any definite conclusions from them. It appears, however, that in the majority septic troubles of some sort were present, either septic pyelonephritis due to infection from the bladder, or septic inflammation in connection with the wound. In a few only is it stated that the wounds healed well without any trouble.

But if the condition of the patient at the time that the operation is performed is borne in mind, it does not seem to me that the occurrence of these symptoms requires any special or unusual explanation. I have many times known symptoms identical in character and mode of onset follow 
simple fractures and even sprains and bruises in old people whose vitality is failing. They begin in the same quiet and insidious way. There is the same variety; sometimes merely a little wandering at night; sometimes childishness; sometimes again wild excitement with hallucinations. They occur in those who have led the most temperate lives as well as in those who have not, and though they nearly always subside in the course of a few days, sometimes in the same way they persist for the rest of life. The cause is the condition of the patient rather than the particular character of the operation or injury, although of course the more serious this may be, the more likely these symptoms are to follow.

Great care, therefore, must be exercised in selecting the cases in which this operation is performed, and in securing a strictly aseptic condition of the wound. If the patient is becoming more and more feeble, especially if the change has been rather rapid of late; if there is distinct evidence of the failure of mental power that occurs so often as age advances; if the heart's action is weak and rapid; if the vessels are markedly atheromatous, or if there is definite pyelonephritis and contracted granular kidney, the friends should be warned beforehand that there is a possibility that these symptoms may set in. As a rule, if septic infection of the wound is avoided, they disappear as the effects of the shock pass off and the patient regains strength; but a few instances are recorded in which the failure of power has continued and even grown worse as time went on.

It is for this reason that in spite of the advantage of having only one incision, I have quite given up the scrotal operation for orchidectomy.

A scrotal wound in an old man is very difficult to keep aseptic. An incision on each side over the external abdominal ring may take a little longer, but the skin can be cleansed much more thoroughly; the testicle, unless it is enlarged or associated with a thickened hydrocele sac can be extracted quite as quickly; and the wound can be sealed with gauze and collodion so as to secure it effectually against subsequent contamination, even if the patient does disturb the outer dressings. For the same reason in many cases I remove one testis first, and then wait, it may be six weeks 
or two months, until the patient has thoroughly recovered, before removing the other.

\section{The Effect of Unilateral Orchidectomy upon Enlargement of the Prostate.}

The arguments in favour of unilateral orchidectomy are not nearly so convincing as those which support the bilateral operation. This is unfortunate, because there can be very little doubt that, if they were equally good, many patients who will not hear of the complete operation being performed until it is well nigh too late, would willingly agree to the partial one.

As I have mentioned already, in speaking of the development of the gland, the prostate is really a double organ, each half originally being developed from the corresponding Wolffian duct. In many of the lower animals the two halves remain distinct. In man they fuse together, and the fusion takes place to a very different degree in different individuals, so that the two halves sometimes remain almost separate from each other, sometimes form a single organ. Experiments upon animals therefore are worthless, so far as it is wished to apply to man any deduction which may be formed from them; and the results obtained by clinical and pathological observation present very great discrepancies.

If one testis fails to develop, owing to non-descent or other causes, occasionally the corresponding half of the prostate and the vesicula seminalis fail too; but not in the majority of instances. In the majority no difference can be detected in the prostate.

If one testis is destroyed in infancy, it may or may not have an effect upon the shape of the gland. I have recorded elsewhere a case in which the complete disappearance of one testis owing to violence in infancy did not prevent the outgrowth of a valvular median lobe in old age.

The same thing holds good if one testis without being absorbed, becomes small and shrunken from attacks of chronic epididymitis. In a few cases, but certainly a small minority, the corresponding lobe of the prostate wastes. On 
the other hand, several instances have been recorded in which it has been distinctly larger than the other.

As might be expected from this, the effect of unilateral orchidectomy is very uncertain, and generally negative, but with a few brilliant exceptions. In one case, for instance, under the care of Dr. Manning of Salisbury, the lobes were unequally enlarged, the left being considerably the bigger of the two. The left testis was removed, with very great but not complete relief. The patient who was 82 years of age died some months later and at the post-mortem it was found that the size of the lobes was completely reversed, the left which had been the larger being much the smaller. And there are a few other similar cases on record. On the other hand, I have operated five times on one side only, removing the second testis six weeks or two months later. The time of course is too short to say definitely that atrophy would not have taken place; as I have shown in speaking of double orchidectomy at least six months and probably a twelvemonth must be allowed before coming to a negative conclusion on this point; but there certainly was no change that I could detect.

As I have mentioned already considerable temporary improvement followed in one of these cases after the first testis was removed; but it disappeared again in the course of a few days. The case finally did exceedingly well.

Unilateral orchidectomy should be reserved for cases in which there is a conspicuous inequality in the size of the lobes, and in which it can be shown that the enlargement is confined to the lateral lobes; but the patient must be distinctly given to understand that the result cannot be guaranteed and that though the operation may be successful, it is more likely that it will not be, and that the second testis will have to be removed later.

The Effect upon the Prostate of Division of the Various Structures contained in the Spermatic Cord.

There is no longer any doubt as to the relation that exists between the normal prostate and the testes. Removal of the 
latter causes atrophy of the former. There may be a few exceptions, but they are not sufficiently numerous to invalidate the general rule. Many of them can be explained. Those that cannot probably will be when our knowledge of the influence which one organ in the body exerts upon the rest becomes more exact.

There can be little doubt, moreover, that the influence of the testes upon the prostate is exerted in some way or other through the instrumentality of the nervous system. The occasional success of unilateral orchidectomy is sufficient to prove this. Unilateral atrophy could not occur if the agent were the cessation of some internal secretion.

The relation that exists between the prostate and the various structures contained in the spermatic cord is much more difficult to estimate. Developmentally, the prostate is in closer association with them than it is with the testes. The prostate is an outgrowth from the Wolffian duct of which the vas deferens is the permanent representative, while the testes are of entirely separate origin. Functionally it is not. Removal of the master organs of the generative system is followed by atrophy of the subordinate structures. They have no further work in the organism and are mere useless appendages. But removal of one of the subordinate structures does not of necessity involve any interference with the function or nutrition of the rest. The vas deferens for instance might be divided or cut without, of necessity, exerting any direct effect upon the prostate. The prostate might continue to work and grow as before.

There are two things that make this enquiry very difficult. The one is that though section of the vas deferens may have no direct effect upon the prostate, it may have one that is indirect. It may affect the nutrition of the testes and this in its turn may affect that of the prostate. The other is that experiments upon animals and especially upon dogs are only to be relied upon in such matters to a very limited extent.

There is no fixed relation, as some experimenters have tried to maintain, between the body-weight of a dog and that of its prostate. The prostate in dogs of the same weight varies very greatly in size. It is not improbable that it may vary in size in individuals at different times of the year. 
Grave changes, such as the complete atrophy which follows double orchidectomy, cannot be mistaken. But when the difference in weight or in size can only be represented by fractions or percentages, the argument is worthless.

The nutrition of the testes, moreover, differs very considerably in men and in dogs. Ligature of the spermatic artery in a dog was shown long ago by Curling to be followed by atrophy. According to Alessandri and others, if all the vessels in the cord are tied, this result is almost invariable. This is certainly not the case in man. I have performed what is known as the open operation for varicocele more than two hundred times, removing the greater portion of the veins and with them the spermatic artery; but so far as I am aware atrophy of the testes has never occurred but once, and in that patient $I$ attribute the result to the wound having become septic and suppuration having set in.

Ligature of the spermatic cord as a whole is generally followed by atrophy of the testis in man, and this in its 'turn by diminution in size of the prostate. The testis swells up, becomes tender for a few days, and then shrinks until it practically disappears. I have performed this operation on several occasions with an excellent result, the wound in each case pursuing an aseptic course and healing without being disturbed; but in one case the testis, though it diminished in size, did not undergo complete atrophy, and the result, so far as the prostatic symptoms were concerned, was only a moderate degree of improvement. It is said by others to have been followed by sloughing of the testis and severe inflammation, but in none of mine was there more than a transient swelling. Section of the cord is followed by the same changes.

Of the structures contained in the cord, the nerves of the spermatic plexus and the vas deferens are the only ones which require separate mention. The effect of ligature of the vessels has been dealt with already.

Section of the nerves.-As the influence of the testes upon the prostate is exerted through the medium of the nervous system, there is some reason for thinking that the effect of section of all the nerves in the cord going to and from the testis would be the same as removal of the testis. Certain 
experiments performed upon dogs by Prjéwalski bear this out, as complete atrophy of the prostate followed.

Section of the nerves alone, however, is not practicable as an operation in man. The matter derives importance from the fact that it may serve to explain the very different results that appear to have followed section and ligation of the vasa deferentia.

Division of the vasa deferentia.-Injury to the vasa deferentia does not affect the testes in man as a rule. Hilton described three cases of subcutaneous rupture of the vas deferens in which, after a transient increase in size, the testis shrank to half its former bulk, but Curling, Astley Cooper, Gosselin, and many others, did not find that section of the vas alone had any permanent effect in the adult. Moreover the diminution in size which occurred in Hilton's cases is not the same as atrophy.

Experiments that have been performed upon animals upon the whole confirm this. The vasa deferentia may be divided without causing atrophy of the testes; but it is essential that the injury should be limited to these structures. If the nerves or the vessels are divided, as well as the vas deferens, atrophy may occur and may be complete.

With regard to any direct influence which the vasa deferentia may possess over the normal prostate in man nothing is known. In all probability they have none. The whole of the structures developed from the Wolffian body may fail upon one side or upon both; but there is no evidence that injury to the vas deferens is followed by any change in the normal prostate.

The results of the experiments that have been performed upon dogs with the view of ascertaining whether the same thing holds good with regard to them are very far from uniform. As I have said already no reliance is to be placed upon differences in the weight of the gland; they are purely conjectural. Nothing can be trusted but definite microscopic evidence of widely spread fatty degeneration. On the whole it seems that a certain degree of atrophy does sometimes follow. Many of the results are negative. Many more do not fulfil the required conditions. But a few are positive. As Albarran has pointed out, the atrophy does not make its 
appearance for some months. It is not nearly so complete when it does occur as it is after excision of the testes. And the greater the care taken in the isolation of the vas, the smaller the result.

This distinctly suggests that the effect upon the prostate is due rather to the division of the nerves or the vessels than to that of the vas, and raises the suspicion that the changes in the prostate are really only secondary to those that have taken place in the testes.

I have operated by this method in fourteen cases of enlargement of the prostate. None of my patients have died from the operation. In the first six I carefully isolated the vas, pulling it up in a loop with an aneurysm needle. A ligature was then placed around the loop, and the convexity cut off. In the rest I have deliberately excised four or five inches of the vas, as much in fact as I could get out of the wound, and have ligatured both ends and removed the intermediate part with all the loose cellular tissue that would adhere to it. This has been done with the object of dividing as many of the nerves of the spermatic plexus as possible.

For the same reason as in orchidectomy I always now give the preference to the inguinal incision. The vas it is true can be isolated by the pressure of the finger and thumb through the skin of the scrotum, and a small incision made directly over it; but this gives no opportunity for dealing with the surrounding tissues in which the nerves are believed to lie, and there is always the risk that the wound, especially in an old man, may become septic.

In my opinion this operation is a much less satisfactory one than orchidectomy and only suited to a very limited class of cases. The chief advantages that it possesses are that a general anæsthetic is not absolutely necessary ; it can be performed under cocaine; and that for sentimental reasons a patient may agree to it at a time when he would not consent to have the major operation performed. The retention of two organs which were probably useless before, and certainly must be after the operation, is sometimes a point to which a patient attaches considerable importance. On the other hand, the result is most uncertain.

I have noticed a slight degree of immediate improvement 
in several cases, but only in those in which there had been recent acute retention of urine. It is due, no doubt to the effect of the operation upon the congestion at the neck of the bladder, as it was in the case of unilateral orchidectomy which I mentioned above; and as also in this case it is of a very transient character, rarely lasting more than two days.

Diminution in size of the enlarged prostate is less frequent and less marked than it is after orchidectomy. It occurred in about half of my cases, but in none of them was it sufficient to justify the term atrophy. In no single case did the prostate shrink until it could only be felt as a small flat mass behind the urethra. There was always a distinct projection to be felt per rectum quite as prominent as that which is produced by the normal prostate.

In one case, in which five inches of the vas deferens were excised on each side, the prostate after a temporary decrease in size began to grow again. Six months after the operation it was decidedly larger than it was before; and the patient was suffering again from hæmaturia and retention of urine. Albarran (Ann. des Mal. des Ovg. Genito urinaives, March, 1898) mentions a similar instance in which two years and a half after the operation the prostate was much larger than it was before. And there are at least two other cases on record in which the growth was not checked.

In five of my cases there was no diminution in size at the end of six months. In two of these the testes did not undergo any particular change. In the other three they became softer and smaller; but they did not atrophy in the sense in which, after injury or inflammation, the testis sometimes atrophies, growing smaller and smaller until the body of the organ disappears completely. When complete atrophy of the testis such as this is induced, the result, so far as the enlarged prostate is concerned, may be more satisfactory. Mere loss of size and consistence, which is all that occurred in the cases in which I divided the vasa deferentia, is clearly not enough.

So far as I can gather from the records of published cases, a certain degree of diminution in size of the enlarged prostate is fairly frequent after section of the vasa deferentia. In most of these, however, the observations are of only a few 
months date. In many, of only a few weeks. Very few say anything about the condition of the gland twelve months after the operation; and as there are some undoubted cases in which the prostate has begun to grow again after a temporary diminution, this is essential before the case can be counted as successful.

I have not been able to find a record of any case giving definite clinical or microscopical proof of atrophy of the enlarged prostate as a consequence of this operation.

In short, section of the vas alone cannot be relied upon to produce any permanent change either in the testis, or in the normal or the enlarged prostate. Excision of a considerable portion of the vas with the soft cellular tissue that surrounds it, is a little more satisfactory. The prostate sometimes diminishes in size; sometimes it continues to grow. The result probably depends upon the extent to which the nerves that supply the testis have been divided with the vas. To ensure a really satisfactory result all the nerves must be cut. In most instances, if not in all, this cannot be done without dividing the vessels as well, for many of the nerves run with them. But this means section or ligation of the whole spermatic cord.

There is no evidence, clinical or experimental, that atrophy of the normal or enlarged prostate (as distinguished from mere diminution in size) can be induced by this operation except as a secondary result of degenerative changes in the testes.*

The degree of permanent relief that has been experienced by the patients upon whom I have operated in this way has varied a great deal. One case as I have mentioned already was better for a while, and then the prostate began to grow again and all the old symptoms returned. Another one I have been unable to trace.

Of the others, so far as the symptoms which may reasonably be assigned to congestion and hyperæmia at the neck of the bladder are concerned, all but three have obtained some

* It is interesting to note in connection with this subject that, according to Bazy, Escat and Chailloux, extirpation of the testis in dogs, leaving the epididymis intact, is followed by rapid and definite atrophy of the prostate, proved by microscopic examination. 
relief. The frequency of micturition has diminished. The patients do not rise quite so often at night. The dysuria and strangury do not appear to cause so much distress as they did before; but three cases have not improved at all, and only three can be said to be free from discomfort. One for instance tells me that he knows by a feeling in his testes when his urine is going to trouble him. The feeling is that of enlargement, and he thinks that they actually do get larger. This I am unable to confirm. Another who is very well satisfied with the result, and who in the nine months that followed the operation put on nearly two stones in weight which he had lost in the previous year, writes to me that he does not pass water so frequently and that he only has to rise twice in the night, but that he cannot wait a moment after he begins to feel the desire come on him. Two others say very much the same. In short, there is a certain degree of relief from the congestive symptoms in the majority, but complete cessation even of these is the exception.

Four of the patients under my care had suffered on previous occasions from complete retention of urine. Since the operation there has been no further trouble of this kind; but as in three of these there had only been a single attack, this does not prove very much.

- A noteworthy difference between this operation and orchidectomy is the extreme slowness with which improvement takes place. Instead of their being a distinct amelioration of the symptoms within the course of a few days or weeks at the most, progress does not often show itself for months and is so slow as to be almost imperceptible. As these patients were all of them taking good care of themselves, and observing strict precautions as regards diet, exposure to cold, and the indiscretions that so frequently cause congestive attacks of the prostate, this raises the question whether a great deal of the improvement, when it did take place, may not have been due to these precautions and to the careful living likely to be followed after an operation, rather than to the operation itself. I am inclined to think that a good deal of allowance must be made for this.

I think there is sufficient evidence to show that attacks of acute congestion are less common and less severe after this 
operation than they were before. But it is very difficult to say whether this partial exemption, is, like the improvement in the general symptoms mentioned above, to be assigned to the altered mode of life and the greater amount of care observed, or to the direct effect of the operation. The complete exemption that generally occurs after orchidectomy is certainly not the rule after division of the vasa.

The patients who are the most likely to derive benefit from this operation are those who bave been the most liable to acute attacks of retention, and the degree of benefit is proportionate to the shortness of time that has elapsed since the latest.

The effect of division of the vasa on the amount of residual urine is even more disappointing. As might be expected from what $I$ have said above with regard to the effect on the size of the prostate, in the majority of my cases the amount has remained about the same. In some it has decreased a little; in others it has increased. In only one has it fallen habitually below one ounce from an average of five or six. Nearly all have to continue to use the catheter more or less frequently according to the amount of urine that the bladder cannot expel. The prostate, as a result of this operation, does not diminish in size sufficiently to enable the bladder, even with the assistance of the abdominal muscles, to empty the post-prostatic pouch. It still remains an obstruction at the neck. The low level urethra which is formed when the prostate really undergoes atrophy never becomes established.

I believe the tendency to cystitis which is so liable to be caused by catheterism is diminished by this operation. This might be expected from the effect that it has upon the congestion at the neck of the bladder. Anything that lessens this lessens the chance of the germs which are introduced with the catheter gaining a foot-hold. If the bladder is emptied regularly and there is no congestion, even pyogenic organisms are not capable of causing much mischief in the bladder. But a much more prolonged series of observations is needed before this can be established.

One good result of this operation is that patients acquire freedom from the attacks of epididymitis which not unfrequently result from the passage of septic instruments up the 
urethra. As a whole, however, division of the vasa can only be regarded as an unsatisfactory substitute for orchidectomy. It has little or no direct effect upon the prostate. Whatever influence it has upon the growth of the prostate is secondary to the changes that are produced in the testes. And although it lessens the congestion of the gland, relieving some of the most distressing symptoms, and very probably checks the liability to subsequent attacks of acute congestion, each of which inflicts increased injury upon the bladder, it is questionable whether, in the majority of instances, a similar result could not be obtained without an operation at all. If milder measures have been tried and have failed, and the suffering of the patient is due not so much of the actual size of the prostate, as to constantly returning attacks of congestion, excision of the vasa with the surrounding cellular tissue, which is certainly a less serious operation than orchidectomy, may be tried. Sometimes it gives a certain measure of relief. Further observation, however, is necessary before it can be definitely stated that even this degree of relief will be permanent. The evidence at present does not justify it.

Ligature of one of the vasa deferentia which has been tried in a few instances has nothing to recommend it. 



\section{INDEX.}

Acidity of urine, $\mathrm{ro}_{4}$

Acid phosphate of soda, ro5

Adrenalin, 157

Adventitious capsule, $2 \mathrm{I}$

Age at which enlargement begins, 35

effect of, on bladder, 56

exemption of old, 37

Albarran, x 19,182

Alcohol, ro2, $x_{4} 6$

Alexander's operation, 167

Alkalies in cystitis, ro5

Alkaline urine, 105

Anatomy of prostate, 8

Antiseptics, 135

Aspiration, 147

Atheroma and enlargement, 39 and renal degeneration, 63

Atony of bladder, 56, 1 ro

Atrophy following partial removal, ${ }_{5} 8$ following castration, 182 division of vasa, 196

Bacterium coli, 97 pyogenes, $6 \mathbf{r}$ mode of entrance of, $\mathbf{I}_{3} \mathbf{I}$

Barling, $x 62$

Baths, 103

Belfield, 36, I54, 157

Belladonna, 104

Benzoic acid, 105

Bladder, alteration in orifice of, 53

atony of, $5^{6}$, x ro

distension of, $\mathbf{I} 43$

drainage of, $x 3^{8}$

effects of age on, 56

catheterism on, 94

enlargement on, 50

hypertrophy of, 55

inflammation of, I3

irritability of, 6o, 126

ligaments of, 2
Bladder, recovery after operation, I62, 185, 196

relations of, $3, I_{5}$

shape of orifice of, 88

sphincter of, $I_{7}$

state of, 93

tapping, $139, x_{4} 8$

tone of, 93, Iro

vessels of, 54

washing out, 133

Boric acid, I05, I32

Boroglyceride, $x \mathbf{x} 6$

Bottini, 172

Bouchon vaginale, 15

Boutonnière, $x_{59}$

Bryson, 182

Buchu, ro6

Calculus, $\mathrm{r}_{5}, 8 \mathrm{r}, \mathrm{I}_{50}$

Cancer of prostate, 82

Capsule of prostate, 4, 21

Caput Gallinaginis, 16

Castration, 179

Catheter fever, i i 7

Catheterisation, frequency of, 124

Catheters, $x \times 3$

cleansing of, I I 5

dangers of, $\operatorname{Ir}_{3}$

effect on bladder of, 94

kinds of, I 13

tying in, 137

Causes of enlargement, 37

Chills, II 7

Chloride of calcium, 149

Chronic inflammation of prostate, 82

Clothing, ro3.

Cocaine, 128

Congestion, 55

effects of, $72,176,181$

Cord, ligature of, 192

Cupping, 146 
Curling, 192

Cystitis, 96, I3 I

drainage in, 138

prevention of, 126

Cystoscope, 89

Cystotomy, perineal, ${ }^{3} 8$

preliminary, 164

supra-pubic, r 39

Development of prostate, II

Diagnosis, $8 \mathrm{r}$

Diet, Ior

Digitalin, $\mathbf{r}_{4} 6$

Dilatation of urethra, ro 8

Distension of bladder, 143

Dittel, 153, 163

Drainage of bladder, 138

apparatus for, $14 \mathrm{I}$

choice of method, 138

perineal, $\mathbf{I} 70$

permanent, 138

Dribbling of urine, 67

Effects of enlargement, 52, 63

Ejaculatory ducts, 3

Electricity, I I2, 173

Enlargement, age of commencement,

35

analogy with uterine fibroids, 37

and atheroma, 39

and inflammation, 60

and renal degeneration, 63,98

as compensation, $4 \mathrm{r}$

causes of, 34

effect on bladder, 52

on kidneys, 63

of castration, $\mathbf{I} 79$

of section of vasa, 193

of pressure, ro7

exemption of old age, 37

frequency of, 30

histology of, 20

influence of, on urethra, 29

on urination, 66

measurement of, 90

nature of, 43

of lateral lobes, 30

of median lobe, 30
Enlargement, rate of, 23

relation of testes to, 43

shape of, 26

symptoms of, 66

varieties of, 26

Ergot, 104

Eucalyptus, 106

Examination of urine, 95

per rectum, 83

per urethram, 89

Exercise, 103

External urethrotomy, I3 8

Faulds, 183

Fever, II7

Frequency of catheterisation, 126 of enlargement, 30

of micturition, 73

Freudenberg, 172

Freyer, 156

Function of prostate, 13

Galvanism, I I 2

Galvano-cautery, $\mathbf{I}^{2} 2$

Germicides, 135

Glandular structure, 8

Griffiths, 9, 44, r82

Growth, rate of, 23

Guaiacol, 128

Guthrie, I 7, I69

Guyon, I16, I29

Hæmaturia, $\mathbf{I}_{4} 8$

Hæmorrhage, 149

Hæmorrhoids, 77

Halle, IIg

Harrison, 4I

Henle's muscle, II

Histology of enlargement, 20

Homology of prostate, 13

Hunter, 177

Hydrastis Canadensis, Io6

Hypertrophy of bladder, 55

Incontinence of urine, 70

Instillation, 129

Intermittent micturition, 67

Involuntary micturition, 70

Iodine terchloride, 135

Iodoform, 135

Irritability of bladder, 126 
Irritability of bladder, treatment of, 128

Kidneys, degeneration of, 98 effect of enlargement on, $\boldsymbol{6}_{3}$

Lateral lobes, enlargement of, 30 frequency of, 33 influence on bladder, 52 treatment of, 160

Lubricants, II6

McGill, 35, I54, I69

McGuire, I4 1

Measurements of urethra, 9I

Median lobe, 5 enlargement of, 27 frequency of, 30 influence of, 35

Melchior, 97, II5, II9

Micturition, frequency of, 66 intermittent, 67 involuntary, 70 nocturnal, 75

Morris, I4I

Mortality after prostatectomy, r6o after orchidectomy, $\mathrm{x} 86$

Muscular theory of enlargement, 4I

Nerves, section of. 192

Nicoll, II6, I64

Nitrate of silver, 135

Nux vomica, In4

Orchidectomy, 179

Orifice of bladder, changes in, 53, 88 action of, $\mathrm{r} 8$

Otis, $15^{6}$

Overflow, 70

Pain, 77

Parker Syms, 167

Pelvic fascia, 2

Perineal distance, 168

drainage, $13^{8}$

incision, 164

prostatectomy, I62

prostatomy, r 70

tapping, 138

Physical characters of enlargement, 25

Piperazine, 105

Plexus, prostatic, 6

Polyuria, 123

Prolapse, 77
Prostate, capsule of, $4,2 I$

concretions, 14

development, II

examination of, 83

function, $\mathrm{r}_{3}$

homologies, 13

influence of testes on, 43

median lobe of, 5

relations of, 2

secretion of, 13

sheath of, 2

size of, I

stroma, 5, 8, 15, 21

structure of, 4

tumours of, 23

vessels, 6

weight, $\mathbf{r}$

Prostatectomy, combined, 164

cases suited to, 157

followed by atrophy, $15^{8}$

mortality after, $16 \mathbf{r}$

perineal, 162

supra-pubic, 154

urethral, $\mathbf{1}_{72}$

Prostatic concretions, $I_{4}$

congestion, 55

glands, origin of, I2

plexus, 8

tumours, 23

utricle, II

Prostatometer, 9I

Prostatomy, 170

Proust, 167

Purgatives, 103

Quinine, 106, 132

Ramm, $\mathbf{x} 78$

Rate of growth, 23

Rectal bag, I54, 139

symptoms, 77

tapping 147

Rectum, examination of, 84

Recurrence, frequency of, ${ }_{5} 8$

Relation to testes, 2, 43

Renal degeneration, causes of, 64

diagnosis, 98

effects of, 63

Residual urine, 68 
Residual urine, amount of, $92, I_{4}, I_{2}$ I cause of, 69

treatment of, II8, I2I

Resins, 106

Retention, 7 $\mathbf{I}$, prevention of, I Io treatment of, 143

Rigors, II7

Robson, Mayo, I55

Rovsing, I I9

Salicylic acid, 135

Salol, x06, 132

Sandal oil, 106, $x_{32}$

Sanmetto, ro6

Secretion of prostate, 14

Sedatives, 127

Senility as cause, 34

Septic cystitis, I3I

nephritis, 122

organisms, II9, I3I

Septicæmia, I $2 \mathbf{I}$

Sexual power, 77

Shape of enlargement, 84

Shattock, 4

Sheath of prostate, 2

Shock, I22

Size of enlargement, 25, 9 I

Sounds, ro7

Spermatic cord, ligature of, Ig2

Sphincter, 17

Sterilisation, II5

Strangury, $98, \mathbf{1} 28$

Stroma of prostate, $5,8,15,21$

Structure of gland, 4

Strychnia, 104, III

Sugar, 105

Suppression of urine, 122

Supra-pubic cystotomy, I39

prostatectomy, 153

tapping, ${ }_{13} 8$

urethra, $\mathbf{x}_{4} \mathrm{I}$

Symptoms of enlargement, 66

Tampon, 157

Tapping, 138

per rectum, 147

Testes, influence of, 44

Thompson, Sir H., 2, 5, 25, 30, 33,

37,139
Tobin, $\mathrm{r}_{5} 6$

Tone of bladder, 93, $x$ 10

Toxæmia, IIg

Trendelenburg, 140, ${ }_{54}$

Trigone, effects of enlargements on, 52

Turpentine, 106

Unilateral orchidectomy, $\mathrm{I} 89$

Ureters, muscles of, 3

Urethra, artificial, I4I

development of, II

dilatation, 128

direction, 87

effects of enlargement on, 52

examination of, 85

length of, 86

maintenance of, 107

measurement of, $9 \mathrm{I}$

position, 2

stretching, Iog

structure, xo

Urethral operations, ${ }^{7} 72$

Urethrotomy, $\mathbf{1 3}^{8}, \mathbf{x}_{59}$

Urinary sediments, 95

fever, $\mathrm{II}_{7}$

Urination, frequency of, 66

Urine, decomposition of, 96

effect on, 78

examination of, 95

incontinence, 70

quantity, 122

reaction, I05

residual, $68,92, \mathrm{II}_{4}, \mathrm{I}_{21}$

retention, $7 \mathrm{I}$, 1 ro

suppression, 123

Urotropin, xo6

Uterus, homology of, 13

Utricle, II

Valvular outgrowths, diagnosis of, 88 treatment of, 156

Vasa deferentia, section of, $x 93$

Veru montanum, Io

Velpeau, 37

Vessels of prostate, 6

Washing out bladder, 133

Watson's tube, $x 70$

Weight of normal gland, $x$

White, $x_{55}, I_{7} 8$

Zuckerkandl, 164 


\section{CATALOGUE OF WORKS}

PUBLISHED BY

\section{H. K. LEWIS, \\ 136 GOWER STREET, LONDON, W.C. ESTABLISHED 1844.}

Telegrams: PUBLICAVIT, LONDON.

Telephone: 10721 CENTRAL.

A. C. ABBOT'T, м.D.

Professor of Hygiene and Bacteriology, University of Pennsylvania.

THE PRINCIPLES OF BACTERIOLOGY : A Practical Manual for Students and Physicians. Sixth Edition, with III Illustrations, 26 being coloured, post 8vo, i2s. 6d. nett.

H. ALDERSMITH, M.B. LOND., F.R.C.s. Medical Officer, Christ's Hospital, West Horsham.

RINGWORM AND ALOPECIA AREATA: Their Pathology, Diagnosis, and Treatment. Fourth Edition, enlarged, with new Illustrations, demy 8 vo, ios. 6d.

W. H. ALLCHIN, M.D. LOND., F.R.C.P.

University Scholar in Medicine; Member of the Senate and late Examiner in Medicine in the University; Life Governor of University Coliege; Senior Physician to the Westminster Hospital.

AN ACCOUNT OF THE RECONSTRUCTION OF THE UNIversity of London. PART I.-From the Foundation of the University to the Appointment of the First Royal Commission, I825 to 1888 . Imperial 8vo, 2s. 6d. net. [fust published.

IZETT ANDERSON, M.D. EDIN.

Extraordinary Member of the Royal Medical Society of Edinburgh; Honorary Member of the Institute of 7 amaica.

YELLOW FEVER IN THE WEST INDIES. Crown 8vo, 3s.6d.

JAMES ANDERSON, M.D., F.R.C.P.

Late Assistant Physician to the London Hospital, \& $r$.

NOTES ON MEDICAL NURSING from the Lectures given to the Probationers at the London Hospital. Edited by E. F. LAMPorT, Associate of the Royal Sanitary Institute. With an Introductory Biographical Notice by the late Sir Andrew Crark, Bart. Third Edition, with Glossary, crown 8 vo, 2s. $6 \mathrm{~d}$.

G. GRANVILLE BANTOCK, M.D.. F.R.c.s. EDIN. Surgeon to the Samaritan Free Hospital for Women and Children.

ON THE TREATMENT OF RUPTURE OF THE FEMALE Perineum Immediate and Remote. Second Edition, with Illustrations, 8vo, 3s. $6 \mathrm{~d}$.

A PLEA FOR EARLY OVARIÓTOMY. Demy 8vo, 2s, 
FANCOURT BARNES, M.D., M.R.C.P.

Physieian to the Chelsea Hospital for Women; Obstetric Physician to the Great Northern Hospital, \&c

A GERMAN-ENGLISH DICTIONARY OF WORDS AND Terms used in Medicine and its Cognate Sciences. Square 12mo, Roxburgh binding, 9 s.

\section{JAMES BARR, M.D.}

Physician to the Northern Hospital, Liverpool; Medical Officer of Her Majesty's Prison, Kirkdale, \&c.

THE TREATMENT OF TYPHOID FEVER, and reports of fifty-five consecutive cases with only one death. With Introduction by Sir W. T. Gairdner, M.D., LL.D., Professor of Medicine in the University of Glasgow. With Illustrations, demy $8 \mathrm{vo}, 6 \mathrm{~s}$.

ASHLEY W. BARRETT, M.B. LOND., M.R.C.S., L.D.S.E. Consulting Dental Surgeon to the London Hospital, and late Lecturer on Dental Surgery in the Medical School; Examiner in Dental Surgery to the Royal College of Surgeons, England.

DENTAL SURGERY FOR MEDICAL PRACTITIONERS AND

Students of Medicine. Fourth Edition, with Illustrations, crown 8vo, 3s. 6d.

[Lewis's Practical Series.] [Fust published.

H. CHARLTON BASTIAN, M.A., M.D., F.R.S., F.R.c.P.

Emeritus Professor of the Principles and Practice of Medicine in University College, London; Consulting Physician to University College Hospital, \&c.

\section{I.}

A TREATISE ON APHASIA AND OTHER SPEECH DEFECTS. With illustrations, medium 8 vo, 15 s.

II.

PARALYSES : CEREBRAL, BULBAR, AND SPINAL. A Manual of Diagnosis for Students and Practitioners. With numerous Illustrations, $8 \mathrm{vo}$, $12 \mathrm{~s} .6 \mathrm{~d}$.

III.

VARIOUS FORMS OF HYSTERICAL OR FUNCTIONAL Paralysis. Demy 8vo, 7 s. 6 d.

W. BATESON, M.A., F.R.S.

MENDEL'S PRINCIPLES OF HEREDITY. A Defonce, with a Translation of Mendel's original Paper on Hybridisation. Crown ovo, $4 \mathrm{~s}$. nett.

W. M. BEAUMONT.

Surgeon to the Bath Eye Infirniary.

THE SHADOW-TEST IN THE DIAGNOSIS AND ESTImation of Ametropia. Post $8 \mathrm{vo}, 2 \mathrm{~s}$. $6 \mathrm{~d}$. 


\section{F. E. BEDDARD, M.A., F.R.s.}

[See Cambridge Biological Series, page 5.

CHARLES E. BEEVOR, M.D. LOND., F.R.C.P.

Physician to the National Hospital for the Paralysed and Epileptic, the Great Northern Central Hospital, and the National Orthopadic Hospital.

DISEASES OF THE NERVOUS SYSTFM. A Handbook for Students and Practitioners. With Illustrations, crown 8vo, 1os. 6d.

[Lewis's Practical Series].

A. HUGHES BENNETT, M.D., M.r.c.P.

Physician to the Hospital for Epilepsy and Paralysis, Regent's Park, \&c.

A PRACTICAL TREATISE ON ELECTRO-DIAGNOSIS IN Diseases of the Nervous System. With Illustrations, 8vo, 8s. 6d.

E. H. BENNETT, ห.D., F.R.c.s.I.

Professor of Surgery, University of Dublin,

D. J. CUNNINGHAM, M.D., F.R.c.s.I.

Professor of A natomy and Chirurgery, University of Dublin.

THE SECTIONAL ANATOMY OF CONGENITAL CGCAL Hernia. With coloured plates, sm. folio, $5 \mathrm{~s} .6 \mathrm{~d}$.

HORATIO R. BIGELOW, M.D.

Permanent Hember of the A merican Medical Association, \&c.

PLAIN TALKS ON ELECTRICITY AND BATTERIES, WITH Therapeutic Index. With Illustrations, crown $8 \mathrm{vo}, 4 \mathrm{~s}$. $6 \mathrm{~d}$.

\section{DRS. BOURNEVILLE AND BRICON.}

MANUAL OF HYPODFRMIC MEDICATION.

Translated from the Second Edition, and Edited, with Therapeutic Index of Diseases, by ANDREW S. CURRIE, M.D. Edin., \&c. With Illustrations, crown 8 vo, 3s. 6d.

RUBERT BOYCE, F.R.s., x.B., x.R.c.s.

Professor of Pathology in University College, Liverpool.

A TEXT-BOOK OF MORBID HISTOLOGY for Students and Practitioners. With r3o coloured Illustrations, royal 8 vo, 3 Is. $6 \mathrm{~d}$. 
A. BROCA, M.D.

Chirurgeon des Hopitaux de Paris, Ec.

AND

F. LUBET-BARBON, M.D,

Ancien interne des Hopitaux de Paris.

MASTOID ABSCESSES AND THEIR TREATMENT.

Translated and edited by Henry J. CurTis, B.S. and M.D. (Lond.), F.R.C.S. (Eng.), formerly Assistant to the Professor of Pathology, University College, London, \&c. With coloured Illustrations, cr. 8vo, $6 \mathrm{~s}$.

O. A. BROWNE, M.A., M.D.

Physician to the Royal Hospital for Diseases of the Chest, \&c.

ANEURYSMS OF THE AORTA. 4to, 2s. 6d.

MARY BULLAR \& J. F. BULLAR, M.B. CANTAB., F.R.C.s. RECEIPTS FOR FLUID FOODS. I6mo, Is.

CHARLES H. BURNETT, A.M., M.D.

Emeritus Professor of Otology in the Philadelphia Polyclinic; Clinical Professor of Otology in the Woman's Medical College of Pennsylvania, \&c.

SYSTEM OF DISEASES OF THE EAR, NOSE AND THROAT.

By 45 Eminent American, British, Canadian and Spanish Authors. Edited by

Charles H. BURnetT. With Illustrations, in two imperial 8 vo vols., half morocco, $48 \mathrm{~s}$. nett.

DUDLEY W. BUXTON, M.D., B.S., M.R.c.P.

Administrator of Anasthetics and Lecturer in University College Hospital; Consulting Anesthetist to the National Hospital for Paralysis and Epilepsy, Queen's Square, \&c.

AN列STHEICS: THEIR USES AND ADMINISTRATION.

Fourth Edition, with Illustrations, crown 8vo. [LEWIS's Practicai. Serif..] [In the Press.

JAMES CALVERT, B.A.. B.SC., M.D. LOND.

Fellow of the Royal College of Fhysicians; Lecturer on Nateria Medica, Pharmacology, and Therapeutics to St. Bartholomew's Hospital.

PRACTICAL PHARMACY AND PRESCRIBING FOR STUdents of Medicine. Being the Course in Use at St. Bartholomew's Hospital. Second Edition, crown 8vo, interleaved, 4 s. $6 \mathrm{~d}$.

HARRY CAMPBELL, M.D., B.S. LOND., F.R.c.P. Physician to the North-West London Hospital.

I.

THE CAUSATION OF DISEASE: An Exposition of the ultimate factors which induce it. Demy $8 \mathrm{vo}, 12 \mathrm{~s} .6 \mathrm{~d}$.

II.

FLUSHING AND MORBID BLUSHING: Their Pathology and Treatment. With plates and wood engravings, royal 8vo, ros. $6 \mathrm{~d}$.

111.

DIFFERENCES IN THE NERVOUS ORGANISATION OF Man and Woman, Physiological and Pathological. Royal 8vo, I5s.

IV.

HEADACHE AND OTHER MORBID CEPHALIC SENSATIONS.

Roval 8vo, I2s. 6 d. 


\section{CAMBRIDGE BIOLOGICAL SERIES.}

(General Editor: A. E. Shipley, M.A., Fellow and Tutor of Christ's College).

THE YERTEBRATE SKELETON. By S. H. REYNOLDS, M. A. Trinity College, Cam. bridge; Lecturer and Demonstrator in Geology and Zoology at University College, Bristol. With numerous Illustrations, crown 8 vo, $12 \mathrm{~s} 6 \mathrm{~d}$.

PRACTICAL MORBID ANATOMY. By H. D. ROLLESTON, M.D., F.R.C.P., Fellow of St. John's College, Cambridge; Assistant Physician and Lecturer on Patholuky, St. George's Hos:pital, London; and A. A. KANTHACK, M.D., M.R.C.P., Lecturer on Pathology, St. Bartholomew's Hospital, London. Crown 8vo, 6s.

ZOOGEOGRAPHY. By F. E. BEDDARD, M.A., F.R.S. With Maps, crown 8vo, $6 \mathrm{~s}$.

PRACTICAL PHYSIOLOGY OF PLANTS. By F. DARIVIN, M.A., F.R.S., and E. H. ACTON, M.A. Third Edition, with Illustrations, crown $8 \mathrm{va}, 4 \mathrm{~s} .6 \mathrm{~d}$.

ELEMENTS OF BOTANY. By F. DARWIN, M.A., F.R.S. Second Edition, with numerous Illustrations, crown 8 vo, 4 s. $6 \mathrm{~d}$.

A MANUAL AND DICTIONARY OF THE FLOWERING PLANTS AND Ferns: Morphology, Natural History and Classification. Alphabeticaliy arranged by J. C. WILLiS, M.A., Director of the Royal Butanic Gardens, Ceylon, \&c. Secund edition, ros. $6 \mathrm{~d}$.

FOSSIL PLANTS, a Manual for Students of Botany and Geology. By A. C. SEWARD, M.A. F.R.S., St. John's College. In two volumes, demy 8vo. Vol. 1., with illustrations, I2s.

[Vol. II. just ready.

PALAEONTOLOGY-INYERTEBRATE. By HENRY WOODS, M.A., F.G.S., University Lecturer in Palao-zoology, Cambridge. Tnird Edition, revised and enlarged, with additional Illustrations, crown $8 v 0,6 \mathrm{~s}$.

OUTLINES OF YERTEBRATE PALAONTOLOGY FOR STUDENTS OF Zoology. By A. S. WUODWARD, M.A. With Illustrations, demy 8vo, I4s.

THE SOLUBLE FERMENTS AND FERMENTATION. By J. REYNOLDS GREEN, Sc.D., F.R.S., L'rolessor of Butany to the Pharmaceutical society of Great Britarr, \&c. Second Edition, 8vo, I2s.

zOOLOGY, an Elementary Textbook. By A. E. SHIPLFY, M.A., Fellow and Tutor of Christ's College, Cambridge. and E. W. MACBRIDE, M.A. (Cantab.), D.Sc. (Lond.). With numerous Illustrations, $8 v 0$, ios. $5 \mathrm{~d}$. net.

GRASSES : a Handbook for use in the Field and Laboratory. By H. MARSHALL WARD, Sc.D., F.R.S., Crown 8vo, 6s.

LECTURES ON THE HISTORY OF PHYSIOLOGY DURING THE SIXteenth, Seventeenth, and Eighteenth Centuries. By Sir M. FOSTER, K.C.B., M.P., M.D., \&c., Professor of Physiology in the University of Cambridge, \&c. 8vo, gs.

THE NATURAL HISTORY OF SOME COMMON ANIMALS. By O. H. LATTFR, M.A., Science Master at Charterhouse School. Crown 8vo, 5s. net.

THE CLASSIFICATION OF FLOWERING PLANTS. By A. B. RENDLE, M.A. (Cantab.), D.Sc. (Lond.), Assistant in Batany, British Museum. Vul. I., Introduction, Gymnosperms, Monocotyledons. 8vo, 10s. 6d. net.

[fust published.

TREES. By H. M. WARD, Sc.D., F.R.S., Professor of Botany in the University of Cambridge, \&c.

Vol. I., Buds and Twigs. Crown 8 vo, 4 s. 6 d. net.

[Fust publishet.

MORPHOLOGY AND ANTHROPOLOGY. By W. L. H. DUCKWORTH, M.A., Fellow ol Jesus College; University Lecturer in Physical Anthropology, \&c. 8vo, r5s. net

[Fust published.

STUDIES FROM THE ANTHROPOLOGICAL LABORATORY. ThE Anatomy School, Cambridge. By the same Author. 8vo, sos. thet.

THE MORPHOLOGY OF PLANTS. By J. C. WILLIS, M.A.

[f ust publishid.

[In the press.

\section{CAMBRIDGE GEOLOGICAL SERIES.}

HANDBOOK TO THE GEOLOGY OF CAMBRIDGESHIRE. For the use of Students. By F. R. COWPER REED, M.A., F.G.S., Assistant to the Woodwardian Professur of Geology. Crown 8vo, 7s. $6 \mathrm{~d}$.

PETROLOGY FOR STUDENTS: An Introduction to the Study of Rocks under the Microscope. By A. HARKER, M.A., F.G.S , Fellow of St. John's College; Demonstrator in Geology (Petrology) in the University of Cambridge. Third Edition, revised, cr. 8vo, 7s. 6d.

THE PRINCIPLES OF STRATIGRAPHICAL GEOLOGY. By J. E. MARR, M.A.,' F.R.S., Fellow and Lecturer of St. John's College, Cambridge, cr. 8vo, 6s,

A TREATISE ON CRYSTALLOGRAPHY. By W. J. LEWIS, M.A. Professor of Mineralogy in the University of Cambridge. Svo, Its. net. 


\section{CAMBRIDGE PHYSICAL SERIES.}

(General Editor: R. T. Glazebrook, M.A., F.R.S., Fellow of Trinity College ; Assistant Director of the Cavendish Laboratory).

HEAT AND LIGHT. By R. T. GLAZEBROOK, M.A. Crown 8vo, 5 s. The two Parts are also nublished separately. HeAT, 3s. Lighr, 3 s.

MECHANICS AND HYDROSTATICS. By the same Author. Crown 8vo, 8s. 6d. Also in separate Parts. Part 1.-DyNAmICS. 4s. Part II.-STATics, 3s. Part IIl.- Hydrostatics, ?s.

A TREATISE ON THE THEORY OF SOLUTION, INCLUDING PHENOMENA OF ELECTROLYSIS. By W. C. D. WHETHAM, M.A., F.R.S., Fellow of Trinity College. 8vo, sos. net.

M ECHANICS. By $]$. COX, M.A., F.R.S.C. 8vo, gs. net

ELECTRICITY AND MAGNETISM. By R. T. GLAZEBROOK, M.A., F.R.S. Crown 8vo,

CONDUCTION OF ELECTRICITY THROUGH GASES. By J. J. THOMSON, D.Sc., LL.D., F.R.S., Fellow of Trinity College, Cambridge. 8vo, $16 \mathrm{~s}$.

RADIO-ACTIYITY. By E. RUTHERFORD, Trinity College, Cambridge, Professor of Phvsics at McGill University, Montreal, 8vo, ros. 6d, net.

TREATISE ON THE THEORY OF ALTERNATING CURRENTS. By A. RUSSELL, M.A., M.I.E.E., late Scholar and Assistant Lecturer, Gonville and Cajus College, C.ambridge. Vol I., svo. i2s.

THE STUDY OF CHEMICAL COMPOSITION, an Account of its Must published. Historical Development. By IDA FREUND, Staff Lecturer and Associate, Newnham College, 8 vo, 38s. net.

SOUND. By J. W. CAPSTICK, M.A., Fellow of Trinity College.

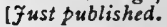
IIn preparation.

\section{J. W. CAPSTICK, м.A.}

[See Cambridge Physical Series, page 6.

ALFRED H. CARTER, M.D., M.sc. LOND.

Fellow of the Royal College of Physicians; Projessor of Medicine, University of Birmingham; Senior Physician to the Queen's Hospitat, Birmingham, \&c.

ELEMENTS OF PRACTICAL MEDICINE.

Eighth Edition, crown 8vo, Ios. 6d.

F. H. CHAMPNEYS, M.A., M.D. oxoN., F.R.C.P.

Physician-Accoucheur and Lecturer on Obstetric Medicine at St. Bartholomew's Hospital : Examiner in Obstetric Medicine in the University of Oxjord, \&c.

I.

LECTURES ON PAINFUL MENSTRUATION. The Harveian Lectures, 18go. Roy. 8vo, 7s. 6d.

EXPERIMENTAL RESEARCHES IN ARTIFICIAL RESPIRAtion in Stillborn Children, and Allied Subjects. Crown 8vo, 3s. 6d.

W. BRUCE CLARKE, M.A., M.B. oxoN., F.R.C.s. Assistant Surgeon to, and Senior Demonstrator of Anatomy and Operative Surgery at St. Bartholomew's Hospital; Surgeon to the West London Hospital, \& $\varepsilon$.

THE DIAGNOSIS AND TREATMENT OF DISEASES OF THE Kidney Amenable to direct Surgical Interference. With lllustrations, demy 8vo, 7 s. 6d.

ALEXANDER COLLIE, M.D. ABERD., M.R.C.P. LOND. Secretary of the Epidemiological Society for Germany and Russia, \&c.

ON FEVERS: THEIR HISTORY, ETIOLOGY, DIAGNOSIS, Prognosis, and Treatment. Illustrated with Coloured Plates, crown 8vo, 8s. 6d. 
E. TREACHER COLLINS, F.R.c.s.

Assistant Surgeon to the Royal London Ophthalmic Hospital, Moorfields; Hunterian Professor, Rojal College of Surgeons, England, $1893-94$.

RESEARCHES INTO THE ANATOMY AND PATHOLOGY OF the Eye. With ro Plates and 28 Figures in the Text, demy 8vo, 6s.

WALTER S. COLMAN, M.D., F.R.C.P. LOND. Assistant Physician to the National Hospital for the Paralysed and Epileptic, Ecc.

SECTION CUTTING AND STAINING: A Practical Introduction to Histological Methods for Students and Practitioners. Second Edition, with Illustrations, crown $8 \mathrm{vo}, 3 \mathrm{~s}$. $6 \mathrm{~d}$.

W. H. CORFIELD, M.A., M.D. oxoN., F.R.C.P. LOND.

Consulting Saritary Adviser to H.M. Office of Works; Hon. Sanitary Adviser to Universitv College Hospital; Projessor of Hygiene and Public Health in University College, London; Medical Officer of Health for St. George's, Hanover Square, \&c.

I."

DWFLIING HOUSES : their Sanitary Construction and Arrangements. Fourth Edition, with Illustrations, crown 8 vo, 3s. 6 d.

II.

DISEASE AND DEFECTIVE HOUSE SANITATION : Boing Two Lectures delivered before the Harveian Society of London. With Illustrations, crown $8 \mathrm{vo}, 2 \mathrm{~s}$.

THE ETIOLOGY OF TYP HOID FEVER AND ITS PREVEN. tion. Being the Milroy Lectures delivered at the Royal College of Physicians, 1902. Demy 8vo, 2s. 6d.

J. COX, M.A., F.R.c.s.

See Cambridge Physical Series, page 6.

SIDNEY COUPLAND, M.D., F.R.c.P.

Physician to the Middlesex Hospital, and Lecturer on Practical Medicine in the Medical School; late Examiner in Medicine at the Examining Board for England.

NOTES ON THE CLINICAL EXAMINATION OF THE BLOOD and Excreta. Third Edition, r2mo, rs. 6d.

\section{CHARLES CREIGHTON, M.A., M.D.}

Formerly Demonstrator of Anatomy in the University of Cambridge.

1.

A HISTORY OF EPIDEMICS IN BRITAIN. Vol. I., From A.D. $66_{4}$ to the Extinction of the Plague. Demy 8vo, 18s. Vol. II., From the Extinction of the Plague to the Present Time. Demy 8vo, 20 .

II.

ILLUSTRATIONS OF UNCONSCIOUS MEMORY IN DISease, including a Theory of Alteratives. Post 8vo, 6s. 
H. RADCLIFFE-CROCKER, M.D. LOND., F.R.C.P.

Physician for Diseases of the Skin in University College Hospital, \&c.

I.

DISEASES OF THE SKIN ; THEIR DESCRIPTION, PATHOlogy, Diagnosis, and Treatment. With special Reference to the Skin Eruptions of Children, and an Analysis of Fifteen Thousand Cases of Skin Disease. Third Edition, with 4 Plates and 112 Illustrations, 2 vols., med. 8 vo, 28s. nett.

\section{II.}

[Now ready.

THE CONDITIONS WHICH MODIFY THE CHARACTERS of Inflammations of the Skin, and their Influence on Treatment. Being the Lettsomian Lectures at the Medical Society of London, 1903. 8vo, 2s. 6d.

[Now ready.

F. DARWIN, M.A., F.R,s.

[See Cambridge Biological Series, page 5.

HERBERT DAVIES, M.D., F.R.c.P.

Late Consulting Physician to the London Hospitat.

THE MECHANISM OF THE CIRCULATION OF THE BLOOD through Organically Diseased Hearts. Edited by ARThur TeMPLer Davies, B.A. (Nat. Science Honours), M.D. Cantab., F.R.C.P., Physician to the Royal Hospital for Diseases of the Chest. Crown 8vo, 3s. $6 \mathrm{~d}$.

HORACE DOBELL, M.D.

Consulting Physician to the Royal Hospital for Diseases of the Chest, \&c.

ON DIET AND REGIMEN IN SICKNESS AND HEALTH, and on the Interdependence and Prevention of Diseases and the Diminution of their Fatality. Seventh Edition, 8vo, 5s. nett.

ROBERT W. DOYNE, r.R.c.s.

Surgeon to the Oxford Eye Hospital; Ophthalmic Surgeon to St. Fohn's Hospital, Cowley, and to the Bourton on Water Cottage Hospital.

NOTES ON THE MORE COMMON DISEASES OF THE EYE. With Test Types, crown 8vo, 29 .

W. L. H. DUCKWORTH, M.A.

[See Cambridge Biological Series, page 5.

PROF. A. DÜHRSSEN, M.D.

Professor in Midwifery and Gynceology in the University of Berlin.

A MANUAL OF GYN ECOLOGICAL PRACTICE FOR STUdents and Practitioners. Second English, translated and edited from the Sixth German edition, by JoHn W. TAYLOR, F.R.C.S., Professor of Gynæcology, the University of Birmingham, and Frederick EDGE, M.D. LoND., M.R.C.P., F.R.C.S., Surgeon to the Wolverhampton and District Hospital for Women. With 125 Illustrations, crown $8 \mathrm{vo}, 3$ s. 6d. nett.

A MANUAL OF OBSTETRIC PRACTICE FOR STUDENTS and Practitioners. Translated and edited from the Sixth German Edition, by JOHN W. TAYLOR and Frederick Edge. With Illustrations, crown 8vo, 3s. od.nett. 
ARTHUR W. EDIS, M.D. LOND., F.R.c.P.

Senior Physictan to the Chelsea Hospital for Women; Late Obstetric Physician to the Middlesex Hospital.

STERILITY IN WOMEN : including its Causation and Treatment. With 33 Illustrations, demy 8 vo, $6 \mathrm{~s}$.

EDWARD J. EDWARDES, M.D. LOND. Member of the Royal College of Physicians, London.

A CONCISE HISTORY OF SMALL-POX AND VACCINATION in Europe. Crown 8vo, 2s. 6d. nett.

W. ELDER, M.D., F.R.C.P. EDIN.

Physician to Leith Hospital.

APHASIA AND THE CEREBRAL SPEECH MECHANISM. With Illustrations, demy $8 \mathrm{vo}$, ros. $6 \mathrm{~d}$.

W. D'ESTE EMERY, M.D., B.Sc. LOND.

Assistant Bacteriologist to the Laboratories of the Royal Colleres of Physicians and Surgeons, London; formerly Lecturer on Pathology and Bacteriology in the University of Birmingham, \&c.

A HANDBOOK OF BACTERIOLOGICAL DIAGNOSIS FOR Practitioners (including Instructions for the Clinical Examination of the Blood). With 2 Coloured Plates and 33 Illustrations, crown 8vo, 5s. 6d.

[Lewis's Practical Series.]

W. SOLTAU FENWICK, M.D., B.s. LOND., M.R.c.P.

Physician to Out-patients at the Evelina Hospttal for Sick Children; Physician to the London Temperance Hosputal.

I.

DISORDERS OF DIGESTION IN INFANCY AND CHILDhood. With Illustrations, demy 8 vo, ros. $6 \mathrm{~d}$.

II.

THE DYSPEPSIA OF PHTHISIS: Its Varieties and Treatment, including a Description of Certain Forms of Dyspepsia associated with the Tubercular Diathesis. Demy 8vo, 6s.

DR. FERBER.

MODEL DIAGRAM OF THE ORGANS IN THE THORAX and Upper Part of the Abdomen. With Letter-press Description. In 4to, coloured, 5s. nett.

G. H. FIN K, M.R.c.s., L.S.A., LOND.

Major, Indian Medical Service (Retired).

CANCER, AND PRECANCEROUS CHANGES, their Origin and

Treatment. $8 \mathrm{vo}, 5 \mathrm{~s}$. 
W. H. RUSSELL FORSBROOK, M.D. LOND., M.R.c.s. Consulting Medical Officer to the Government of the Cape of Good Hope; formerly Surgical Registrar to Westminster Hospital.

A DISSERTATION ON OSTEO-ARTHRITIS. Demy 8vo, 5 s.

SIR M. FOSTER, к.с.в., м.P., м.р., етc.

[See Cambridge Biological Series, page 5.

J. MILNER FOTHERGILL, M.D., M.R.C.P.

Late Physician to the City of London Hospital for Diseases of the Chest, Victoria Park, \&c. I.

INDIGESTION AND BILIOUSNESS. Second Edition, post 8vo, 7s. 6d. 11.

GOUT IN ITS PROTEAN ASPECTS. Post 8vo, 7s. 6d. III.

THE TOWN DWELLER: His Needs and His Wants. With an Introduction by Sir B. W. RichaRdson, M.D., LL.D., F.R.S. Post 8vo, 3s. 6d.

R. HINGSTON FOX, M.D. BRUX., M.R.C.P. LOND.

WILLIAM HUNTER: Anatomist, Physician, Obstetrician, (1718-1783). With notices of his friends CULLEN, SMELLIE, Fothergill and Barllie. With seven Portrait.Prints, Chronological Chatt of Lite and Times, and View of Hunter's Birthplace, 8vo, 4s. 6d. nett.

IDA FREUND.

[See Cambridge Physical Series, page 6.

PROFESSOR DR. PAUL FÜRBRINGER.

Director of the Friedrichshain Hospital, Berlin, \&c.

TEXT-BOOK OF DISEASES OF THE KIDNEYS AND Genito-Urinary Organs. Translated by W. H. GilberT, M.D., Physician in Baden-Baden, \&c. Vol. I., demy 8vo, 7s. 6d. Vol. 1I., demy 8vo, Ios. 6d.

\section{SIR DOUGLAS GALTON.}

Late Royal Engineers, K.C.B., Hon. D.C.L., LL.D., F.R.S., Assoa Inst. C.E., M.I.Mech.E., F.S.A., F.G.S., F.L.S., F.C.S., F.R.G.S., \&̈c.

HEALTHY HOSPITALS. Observations on some points connected with Hospital Construction. With lllustrations, 8vo, ros. 6d.

JOHN HENRY GARRETT, м.D.

Licentiate in Sanitary Science and Diplomate in Public Health, Universities of Durham and Cambridge, \&c.

THE ACTION OF WATER ON LEAD; being an inquiry into the Cause and Mode of the Action and its Prevention. Crown 8vo, $4 \mathrm{~s}$. 6d.

ALFRED W. GERRARD, F.c.s.

Examiner to the Pharmacewtical Society, \&c.

ELEMENTS OF MATERIA MEDICA AND PHARMACY. With Supplement (189o). Crown 8vo, 8s. 6d. 
R. T. GLAZEBROOK, M.A., F.R.s.

[See Cambridge Physical Series, page 6.

E. W. GOODALL, M.D. LOND.

Medical Superintendent of the Eastern Hospital of the Metropolitan Asylums Board, formerly Medical Registrar to Guy's Hospital,

AND

J. W. WASHBOURN, c.M.G., M.D. LOND.

Fellow of the Royal College of Physicians; Physician to the London Fever Hospital ; Assistant Physician to Guy's Hospital, and Lecturer in the Medical School.

A MANUAL OF INFECTIOUS DISEASES. Illustrated with Plates, Diagrams, and Charts, demy $8 \mathrm{vo},{ }_{5} \mathrm{~s}$.

JAMES F. GOODHART, M.D. ABERD., F.R.C.P.

Physician to Guy's Hospital, and Consulting Physician to the Evelina Hospital for Sick Children.

ON COMMON NEUROSES : or the Neurotic Flement in Disease and its Rational Treatment. Second Edition, crown 8vo, 3s. 6d.

GEORGE M. GOULD, A.M., M.D.

Ophthalmic Surgeon to the Philadelphia Hospital, \&c.

THE STUDENT'S MEDICAL DICTIONARY : including all the words and phrases generally used in Medicine, with their proper pronunciation and definitions, based on recent medical literature. Eleventh Edition, with elaborate Tables and many Illustrations, $8 \mathrm{vo}, 14 \mathrm{~s}$. nett.

II.

A POCKET MEDICAL DICTIONARY, Giving the Pronunciation and Definition of 30,000 of the Principal Words used in Medicine and the Collateral Sciences. Fourth Edition, bound limp, 32mo, 5s. nett.

SIR W. R. GOWERS, M.D., F.R.c.P., M.R.c.s.

Physician to University College Hospital, \&c.

DIAGRAMS FOR THE RECORD OF PHYSICAL SIGNS. In books of 12 sets of figures, $1 \mathrm{~s}$.

LANDON C. GRAY, M.D.

Professor of Nervous and Mental Diseases in the New York Polyclinic; Visiting Physician to St. Mary's Hospital, \&c.

A TREATISE ON NERVOUS AND MENTAL DISEASES FOR Students and Practitioners of Medicine. With 168 illustrations, 8vo, 2Is.

WILLIAM GRAY, M.D., c.M. (EDIN.).

INFLUENZA, with Special Reference to some Peculiar Sym. ptoms. 8vo, 3s. 6d. 
J. REYNOLDS GREEN, sc.D., F.R.s.

[See Cambridge Bioloyical Series, page: 5 .

A. HILL GRIFFITH, M.D.

Surgeon, Manchester Rosal Eye Hospital.

\section{THE DIAGNOSIS OF INTRA-OCULAR GROWTHS. With} wuodcuts, ovo, is. $6 \mathrm{~d}$.

DR. JOSEF GRUBER.

Professor of Otology in the University of Vienna, Etc.

A TEXT-BOOK OF THE DISEASES OF THE EAR.

Translated from the Second German edition, and Edited, with additions, by Edward Law, M.D., C.M. Enin., M.R.C.S. Exg., Surgeon to the London Throat Hospital for Diseases of the Throal, Nose and Ear; and Coleman Jewell, M.B. LoND., M.R.C.S. ENG., late Surgeon ald Pashologist to the London Throat Hospital. Second Engli:h Edition, with 165 lllustrations, and 70 coloused figures on 2 lithographic plates, royal $8 \mathrm{vo}, 28 \mathrm{~s}$.

F. DE HAVILLAND HALL, M.D., F.R.C.P. LoND.

Physician to the Westminster Hospital; President of the Laryngological Society of London,

HERBERT TILLEY, M.D., B.S. LOND., F.R.c.s. ENG.

Surgeon to the Throat Hospital, Golden Square; Lectureron Diseuses of the Nose and 1 hroat, London Post-Gradtiate College und P'olycline.

DISEASES OF THE NOSE AND THROAT. Second edition, thoroughly revised, with 2 coloured Platcs and 80 Illustrations, crown 8 vo, ros. 6d.

[Lewis's Practical Serdes].

H. J. HAN.SEN.

AND

W. SORENSEN.

Fellow Royai Duntsh Academy, Copenhagen.

ON TWO ORDERS OF ARACHNIDA OPILIONES. Especially the Sub-order, Cyphophthalmi and Ricinuler, namely the Cryptostemmatsidae. With 9 plates, 4 to, i5s. nett.

[Fust published.

A. HARKER, M.A., F.g.s. .

[See Cambridge Geological Series, page 5.

VINCENT DORMER HARRIS, M.D. LOND., F.R.C.P. Physician to the City of London Hosfital for Disiases of the Chest, Victoria Park; Examiting I'hysician to the Rojal Nutional Huspital for Consumption and Liseases of the Chest, Ventnor, Ec., AND

EDWIN CLIFFORD BEALE, M.A., M.в. CANTAB., F.R.c.P. Physictan to the City of Lonuon Hospital for Diseases of the Chest, Vuctoria Park, and to the Great Northern Central Hospital, \&c.

THE TREATMENT OF PULMONARY CONSUMPTION.

A Practical Manual. Crown 8vo, ios. 6d. [Lewis's Practical Series].

\section{Drs. HARVEY and DAVIDSON'S}

SYLLABUS OF MATERIA MEDICA. Revised in accordance with the "British Pharnaconceia," 1898, by William Martindale, F.L.S., F.C.S., Member of Council of Pharmaceutical Society, and late Examiner; Joint Author of "The Extra Phamacopeia." Tenth Edition, foolscap ı6no, Is. ncıt. 


\section{W. S. HEDLEY, M.D.}

Medical Officer in charge of the Electro-Therapeutic Department of the London Hospital.

THE HYDRO-ELECTRIC METHODS IN INEDICINE.

Second Edition, with Illustrations, demy 8 vo, 4 s. 6d.

II.

CURRENT FROM THE MAIN: The Medical Employment of Electric Lighting Currents. With Illustrations, demy 8vo, 2s. 6d. III.

PRACTICAL MUSCLE-TESTING; AND, THE TREATMENT of Muscular Atrophies. With Illustrations, demy 8vo, 3s. $6 \mathrm{~d}$.

H. HELBING, F.c.s.

MODERN MATERIA MEDICA: For Medical Men, Pharmacists, and Students. Fourth Edition, 8vo, 8s. nett.

HERBERT T. HERRING, м.в., в.s. (DURH.), M.R.c.s.

THE STERILISATION OF URETHRAL INSTRUMENTS, and their Use in some Urinary Complaints. With lllustrations, demy $8 \mathrm{vo}, 5 \mathrm{~s}$.

[Now ready.

C. HIGGENS, F.R.c.s.

Ophthalmic Surgeon to Guy's Hospital; Lecturer on Ophthalmology at Guy's Hospital Medical School.

A MANUAL OF OPHTHALMIC PRACTICE.

Second Edition, revised and edited by A. W. Ormond, F.R.C.S.E., Assistant Surgeon, Royal Eye Hospital, Southwark, \&c. With 66 Illustrations, crown 8vo, 7 s. $6 \mathrm{~d}$.

[Lewis's Practical Series].

BERKELEY HILL, M.B. LOND., F.R.c.s.

Professor of Clinical Surgery in University College; Surgeon to University College Hospital and to the Lock Hospital;

ARTHUR COOPEER, L.R.c.P., M.R.C.S.

Surgeon to the Westminster General Dispensary.

SYPHILIS AND LOCAL CONTAGIOUS DISORDERS.

Second Edition, entirely re-written, royal 8vo, i8s.

JAMES HINSHELWOOD, M.A., M D., F.F.P.S. GLAS. Surgeon to the Glasgow Eye Infirmary.

LETTER-, WORD- AND MIND-BLINDNESS.

Crown 8 vo, 3 s.

FROM HOSPITAL WARD TO CONSULTING ROOM, with Notes by the Way; a Medical Autobiography. By a Graduate of the London University, Post 8 vo, 3s. $6 \mathrm{~d}$.

GEORGE LINDSAY JOHNSON, м.A., м.в., в.с. cANTAB. Clinical Assistant, late House Surgeon and Chloroformist, Royal Westminster Ophthalmic Hospital, for.

A NEW METHOD OF TREATING CHRONIC GLAUCOMA, based on Recent Researches into its Pathology. With Illustrations and coloured frontispiece, demy $8 \mathrm{vo}, 3 \mathrm{~s} .6 \mathrm{~d}$. 
H. LEWIS JONES, M.A., M.D., F.R.C.P.

Medical Officer in charge of the Electrical Department in St. Bartholomew's Hospital; President of the British Electro-Therapeutic Society, \&c.

MEDICAI ELECTRICITY. A Practical Handbook for Students and Practitioners. Fourth Edition, thoroughly revised and enlarged, with 12 plates and 168 Illustrations, demy $8 \mathrm{vo}, \mathrm{r} 2 \mathrm{~s} .6 \mathrm{~d}$. nett.

[Lewis's Practical Series]. [Fust published.

L. VERNON JONES, M.D.

GONORRHGAI ARTHRITIS, its Pathology, Symptoms, and Treatment. With Illustrations, crown 8 vo, 2s. $6 \mathrm{~d}$.

T. N. KELYNACK, M.D.

Pathologist to the Manchester Royal Infirmary; Demonstrator and Lecturer on Pathology in the Owens College.

A CONTRIBUTION TO THE PATHOLOGY OF THE VFRmiform Appendix. With Illustrations, large 8vo, 10s. $6 \mathrm{~d}$.

HENRY R. KENWOOD, м.B., D.P.H., F.c.s.

Professor of Hygiene and Public Health at University College, London, and Medical Officer of Health and Public Analyst for Stoke Newington and Finchley.

PUBLIC HEALTH IABORATORY WORK. The part on Public Health Bacteriological Work contributed by W. G. SAvage, M.D., B.Sc., D.P.H., Medical Officer of Health, Colchester, formerly Assistant to the Professor of Pathology, with Charge of the Bacteriological Department, University College, London, \&c. Third Edition, with Illustrations, cr. 8vo, Ios. 6d.

[Lewis's Practical Series].

NORMAN KERR, M.D., F.L.S.

President of the Society for the Study of Inebriety; Consulting Physiczan, Dalrymple Home for Inebriates, etc.

INEBRIETY OR NARCOMANIA: its Etiology, Pathology,

Treatment, and Jurisprudence. Third Edition, 8vo, $7 \mathrm{~s}$. 6d. nett.

S. A. KNOPF, M.D.

Physician to the Lung Department of the New York Throat and Nose Hospital; formerly Assistant Physician 30 Professor Dettwenler, Falkenstein Sanatorium, Germany, Ec.

PULMONARY TUBERCULOSIS: its Modern Prophylaxis and the Treatment in Special Institutions and at Home. With 76 Illustrations, royal $8 \mathrm{vo}, \mathrm{r}_{4} \mathrm{~s}$. nett.

DR. PHILALETHES KUHN.

Staff Surgeon to the Imperial Troops of the South West African Protectorate.

INOCULATION AGAINST MALARIA.

Translated by H. A. NesBit, M.A., with a Table of Curves. Svo, 2s. nett. 


\section{F. CHARLES LARKIN, F.R.c.s. ENG. Surgeon to the Stanley Hospital; late Assistant Lectuter in Physiology an University
College, Liverpool, AND \\ RANDLE LEIGH, M.B., B.Sc. LOND. Semior Demonstrator of Physiology in University College, Liverpool.}

OUTLINES OF PRACTICAL PHYSIOLOGICAL CHEMISTRY. Second Edition, with Illustrations, crown 8vo, paper 2s. 6d. rett, or cloth 3 s. nett.

O. H. LATTER, M.A.

[See Cambridge Biological Series, page 5.

\section{J. WICKHAM LEGG, F.r.c.P.}

Formerly Assistant Physician to Saint Bartholomew's Hospital, and Lecturer on Pathological A natomy in the Medical School.

I.

ON THE BILE, JAUNDICE, AND BILIOUS DISEASEB.

With Illustrations in chromo-lithography, roy. 8vo, 25s.

A GUIDE TO THE EXAMINATION OF THE URINE.

Sevent 2 Edition, edited and revised, by H. LEwIS Jones, M.D., M.A., F.R.C.P., Medical Officer in charge of the Electrical Department in St. Bartholomew's Hospital. With Illustrations, fcap. 8vo, 3s. 6d.

ARTHUR H. N. LEWERS, M.D. LOND., F.R.c.P. LOND.

Senior Obstetric Physician to the London Hospital, and Leturer on Midwifery, London Hospital Medical School; Examiner in Obstetric Medicine to the University of London; Examiner in Midwifery and Diseases of Women at the Conjoint Board of the Royal College of Phystcians of London, and of the Royal College of Surgeons of England, \&c. I.

CANCER OF THE UTERUS: A Clinical Monograph on its Diagnosis and Treatment. With the After Results in Seventy-Three Cases Treated by Radical Operation. With 3 coloured Plates and 5 I original Illus. trations, $8 \mathrm{vo}$, Ios. 6d. nett.

11.

A PRACTICAL TEXTBOOK OF THE DISEASES OF WOMEN. Sixth Edition, with 166 Illustrations and 4 coloured Plates, crown 8vo, Ios. 6d.

[LEwis's Practical Sir.rik.s]. [Fust fublished.

PROF. W. J. LEWIS, M.A.

[See Cambridge Geologica] Series, page 5.

LEWIS'S POCKET MEDICAL VOCABULARY. Second Edition, thoroughly revised, $32 \mathrm{mo}$, roan, $3 \mathrm{~s} .6 \mathrm{~d}$.

ROBERT LINDSAY, A.M., M.B., F.R.c.s.E. Retired Surgeon, A rmy Medical Department.

AN ESSAY ON MALARIA AND ITS CONSEQUENCES.

Crown 8vo, 4s. 
A. W. MACFARLANE, M.D., F.R.C.P. EDIN.

Examiner in Medical furisprudence in the University of Glasgow; Honorary Consulting Physician (Late Physician) Kilmarnock Infirmary.

INSOMNIA AND ITS THERAPEUTICS. Medium 8vo, 12s. 6d.

WILLIAM A. M‘KEOWN, M.D., м.сн.

Surgeon to the Ulster Eye, Ear, and Throat Hospital, Belfast; Member of the Senate of the Royal

University of Ireland; Lecturer on Ophthalmology and Otology, Queen's College, Belfast.

A TREATISE ON "UNRIPE" CATARACT, and its Successful Treatment by Operation, with Tables comprising ${ } 5_{5}$ Cases. With Illustrations, royal $8 \mathrm{vo}$, 12s. 6d. nett.

DONALD J. MACKINTOSH, м.в., м.v.o.

Medical Superintendent, Western Infirmary, Glasgow.

SKIAGRAPHIC ATLAS OF FRACTURES AND DISLOCAtions, with Notes on Treatment, for the use of Students. With 80 plates, demy. $4^{\text {to, }}$ I2s. 6d. nett.

\section{J. M. H. MACLEOD, M.A., M.D., M.R.C.P.}

Assistant Physician for Diseases of the Skin, Charing Cross Hospital; Physician for Diseases of the Skin, Victoria Hospital for Children; Lecturer on Skint Diseases, London Schocl of I ropical Medicine.

PRACTICAL HANDBOOK OF THE PATHOLOGY OF THE Skin. An Introduction to the Histology, Pathology, and Bacteriology of the Skin, with Special Reference to Technique. With 40 Plates, 8 being in Colours, from original Drawings, demy 8vo, 15 s. nett.

JOHN MACPHERSON, M.D.

Inspector-General of Hospitals H.M. Bengal Army (Retired).

ANNALS OF CHOLERA FROM THE EARLIEST PERIODS to the Year $18 \mathrm{r} 7$. With a map. Demy $8 \mathrm{vo}, 7 \mathrm{~s} .6 \mathrm{~d}$.

\section{A. COWLEY MALLEY, B.A., M.в., B.ch.1 т.c.D.}

PHOTO-MICROGRAPHY ; including a description of the Wet Collodion and Gelatino-Bromide Processes, together with the best methods of Mounting and Preparing Microscopic Objects for Photo-Micrography. Second Edition, with Photographs and Illustrations, crown 8 vo, $7 \mathrm{s.} 6 \mathrm{~d}$.

\section{SIR PATRICK MANSON, K.C.M.G., M.D., LL.D.}

\section{THE FILARIA SANGUINIS HOMINIS; AND CERTAIN} New Forms of Parasitic Disease in India, China, and Warm Countries. Illustrated with Plates and Charts, 8 vo, Ios. $6 \mathrm{~d}$.

J. E. MARR, M.A.

[See Cambridge Geological Series, page 5. 
JEFFERY A. MARSTON, M.D., C.B., F.R.C.s., M.R.c.P. LOND. Surgeon General Medical Staff (Retired).

NOTES ON TYPHOID FEVER: Tropical Life and its Sequelæ. Crown 8vo, 38. 6d.

\author{
VIILLIAM MARTINDALE, F.L.s., F.c.s. \\ Late President and Examiner of the Pharmaceutical Society, \\ AND \\ W. WYNN WESTCOTT, M.B. LOND., D.P.H. \\ H.M.'s Coroner for North-East London.
}

THE EXTRA PHARMACOPCEIA. Eleventh Edition, revised by W. HARRISON MARTINDALE, PH.D., F.C.S., and W. WYNN WeSTcotT, M.B., \&c., limp roan, med. $24 \mathrm{mo}, 9 \mathrm{~s}$. 6 d. nett.

\title{
WILLIAM MARTINDALE, F.c.s. \\ Late Examiner of the Pharmaceutical Soctety, \&c.
}

I.

COCA, AND COCAINE : their History, Medical and Economic Uses, and Medicinal Preparations. Fourth Edition, fcap. 8vo, 2s.

ANALYSES OF TWELVE THOUSAND PRESCRIPTIONS : being Statistics of the Frequency of Use therein of all Official and Unofficial Preparations. Fcap. 4to, 2s. 6d. nett.

\section{MATERIA MEDICA IABELS.}

Adapted for Public and Private Collections. Compiled from the British Pharmacopceia of 1898 and other sources. The Labels are arranged in Two Divi, sions:-

Division I.-Comprises Chemical Materia Medica, including Alcohols, Alka. loids, Sugars, and Neutral Bodies.

Division II.-Comprises, with few exceptions, Substances of Organized Struc. ture, obtained from the Vegetable and Animal Kingdoms.

On gummed paper, r2s. 6d. nett.

${ }^{*} *^{*}$ Specimens of the Labels, of which there are about 500, will be sent on application.

\section{S. E. MAUNSELL, L.R.c.s.I.}

Surgeon-Major, Medical Staff.

NOTES OF MEDICAL EXPERIENCES IN INDIA PRINCIpally with Reference to Diseases of the Eye. With Map, post 8vo, 3s. 6d.

C. W. MANSELL MOULLIN, M.D. oxoN., F.R.c.s.

Surgeon to and Lecturer on Surgery at the London Hospital; Examiner in Surgery at the University of Oxford, \&c.

\section{INFLAMMATION OF THE "BLADDER AND URINARY} Fever. 8vo, 5 s.

FNLARGEMENT OF THE PROSTATE: its Treatment and Radical Cure. Third edition, with plates, 8vo, 6s. [fust published,

SPRAINS; THEIR CONSEQUENCES AND TREATMENT. Second Edition, crown $8 \mathrm{vo}, 4 \mathrm{~s}$. 6d. 
GEORGE R. MURRAY, M.A., M.D. CAMB., F.R.C.P.

Heath Professor of Comparative Pathology in the University of Durham; Physician to the Royal Infirmary, Newcastle.

DISEASES OF THE THYROID GLAND. Part I., Myxadema and

Cretinism. With 25 Illustrations, demy $8 \mathrm{vo}, 7 \mathrm{~s} .6 \mathrm{~d}$.

WILLIAM MURRAY, M.D., F.R.C.P. LOND.

ROUGH NOTES ON REMEDIES. Fourth Edition, with additional Chapter on Rothbury as a Health Resort. Crown 8 vo, 4 s. nett.

II.

ILLUSTRATIONS OF THE INDUCTIVE METHOD IN MEDIcine. Crown 8 vo, 3 s. $6 \mathrm{~d}$.

WILLIAM MURRELL, M.D., F.R.C.P.

Physician to, and Lecturer on Clinical Medicine and Foint Lecturer on the principles and Practice of Medicine at, the Westminster Hospital; Examiner in Materia Medica in the University of Glasgow.

WHAT TO DO IN CASES OF POISONING. Ninth Edition, royal $32 \mathrm{mo}, 3 \mathrm{~s} .6 \mathrm{~d}$.

GEORGE OLIVER, M.D. LOND., F.R.C.P. LOND.

I.

BLOOD-PRESSURE AND TISSUE-LYMPH CIRCULATION : The Oliver-Sharpey Lectures, 1904, with addenda.

[Preparing.

II.

A CONTRIBUTION TO THE STUDY OF THE BLOOD AND BLOOD-PRESSURE; founded on Portions of the Croonian Lectures delivered before the Royal College of Physicians, London, I896, with Considerable Extensions. With Illustrations, demy 8 vo, $7 \mathrm{~s} .6 \mathrm{~d}$.

III.

PULSE-GAUGING : A Clinical Study of Radial Measurement and Pulse Pressure. With Illustrations, fcap. 8vo, 3s. 6d.

IV.

HARROGATE AND ITS WATERS: Notes on the Climate of Harrogate, and on the Chemistry of the Mineral Spring. With Map of the Wells, crown $8 \mathrm{vo}, 2 \mathrm{~s} .6 \mathrm{~d}$.

ON BEDSIDE URINE TESTING: a Clinical Guide to the Observation of Urine in the course of Work. Fourth Edition, fcap. 8vo, 3s. $6 \mathrm{~d}$.

- DR. A. ONODI.

Lecturer on Rhino-Laryngology in the University of Budapest.

THE ANATOMY OF THE NASAL CAVITY, AND ITS ACCESsory Sinuses. An Atlas for Practitioners and Students. Translated by St Clarr Thomson, M.D. Lond., F.R.C.S. EnG., M.R.C.P. Lond. With plates, small 4to; 6s. nett. 
SAML. OSBORN, F.R.c.s.

Knight of Grace of the Order of St. Fohn of Ferusalem; Lecturer and Examiner to the St. Fohn A mbulance Association; Surgeon to the Hospital for Women, Soho Square, Ec.

AMBULANCE LECTURES : FIRST AID TO THE INJURED. Fourth Edition, with Illustrations, fcap. 8 vo, 2 s.

AMBULANCE IECTURES: HOME NURSING AND HYgiene. Third Edition, with Illustrations, fcap. 8vo, 2s.

WILLIAM OSLER, M.D., F.R.C.P. LOND., F.R.S.

Regius Professor of Medicine, University of Oxford; President of the Association of American Physicians; Professor of Medicine, Fohns Hopkins University, and Physician-in-Chief fohns Hopkins Hospital, Baltimore.

I.

ÆQUANIMITAS. With other Addresses to Medical Students, Nurses, and Practitioners of Medicine, post 8vo, 7s. 6d. [Fust published.
[

II.

ON CHOREA AND CHOREIFORM AFFECTIONS. Large 8vo, 5 s.

THE CEREBRAL PALSIES OF CHILDREN. A Clinical study from the Infirmary for Nervous Diseases, Philadelphia. Demy 8vo, $5 \mathrm{~s}$.

WILLIAM OSLER, M.D., F.R.S., F.R.C.P. LOND. AND

THOMAS MCCRAE, M.B. TOR., L.R.C.P. LOND.

of the fohns Hopkins Hospital, Baltimore.

CANCER OF THE STOMACH ; a Clinical Study. With Illustrations, med. $8 \mathrm{vo}, 6 \mathrm{~s}$.

KURRE W. OSTROM.

Instructor in Massage and Swedish Movements in the Philadelphia Polyclinic and College for Graduates in Medicine.

MASSAGE AND THE ORIGINAL SWEDISH MOVEMENTS; their application to various diseases of the body. Fifth Edition, with I05 Illustrations, crown $8 \mathrm{vo}, 3 \mathrm{~s}$. $6 \mathrm{~d}$. nett.

CHARLES A. PARKER. F.R.c.s. EDIN.

Assistant Surgeon to the Hospital for Diseases of the Throat, Golden Square, London.

POST-NASAI, GROWTHS. Demy 8vo, 4s. $6 \mathrm{~d}$. 
ROBERT W. PARKER.

Serior Surgeon to the East London Hospital for Children; Surgeon to the German Hospital.

DIPHTHERIA: ITS NATURE AND TREATMENT, WITH Special Reference to the Operation, After-Treatment, and Complications of Tracheotomy. Third Edition, with Illustrations, 8vo, 6s.

LOUIS PARKES, M.D., D.P.H. LOND. UNIV.

Fellow of the Sanitary Institute; Lecturer on Pullic Health at St. George's Hospital Medical School; Medical Officer of Health for Chelsea,

AND

HENRY R. KENWOOD, M.B., D.P.H., F.c.s.

Fellow of the Sanitary Institute and Member of the Board of Examiners; Professor of Hygiene and Public Health at University College, London, oc.

HYGIENE AND PUBLIC HEALTH. Second edit., with 88 Illustrations, crown 8 vo, I2s.

[Lewis's Practical Series.]

LOUIS PARKES, M.D., D.P.H. LOND. UNIV.

INFECTIOUS DISEASES, NOTIFICATION AND PREVENtion. Fcap. 8vo, cloth, 2s. $6 \mathrm{~d}$; roan, 4 s. $6 \mathrm{~d}$.

LESLIE PHILLIPS, м.D.

Surgeon to the Birmingham and Mrdland Skin and Lock Hospital.

MEDICATED BATHS IN THE TREATMENT OF SKIN DISeases. Crown 8vo, 4s. 6d.

\section{HENRY G. PIFFARD, A.M., M.D.}

Clinical Professor of Dermatology, University of the City of New York; Surgeon in Charge of the New York Dispensary for Diseases of the Skin, \&c.

A PRACTICAL TREATISE ON DISEASES OF THE SKIN. With 50 full page Original Plates and 33 lllustrations in the Text, 4tu, $£ 2$ I2s. 6d. nett.

J. E. PLATT, M.s. LoND., F.R.C.s.

Honorary Surgeon to the Hulme Dispensary; Surgical Officer to the Cancer Hospital, Manchester, \&c.

A CONTRIBUTION TO THE SURGERY OF FRACTURES and Dislocations of the Upper Extremity, based upon an analysis of about 700 Consecutive Cases observed at the Manchester Royal Infirmary. With Illustrations, royal 8 vo, Ios.

G. V. POORE, M.D., F.R.C.P.

Professor of Medical furisprudence, University College; Assistant Physician to, and Physician in charge of the Throat Department of, University College Hospital.

LECTURES ON THE PHYSICAL EXAMINATION OF THE Mlouth and Throat. With an Appendix of Cases. 8vo, 3s. $6 \mathrm{~d}$. 
SIR RICHARD DOUGLAS POWELL, Bart., M.D. LoND., F.R.c.P. Physician Extra-ordinary to His Majesty the King; Physician to the Middlesex Hospital; Consulting Hhysician to the Brompton Hospital, Ec.

THE LUMLEIAN LECTURES ON THE PRINCIPLES WHICH Govern Treatment in Diseases and Disorders of the Heart. With culoured Diagrams, demy $8 \mathrm{vo}, 6 \mathrm{~s}$.

BY THE SAME AUTHOR

DISEASES OF THE LUNGS AND PLEUR坐, INCLUDING Consumption. Fourth Edition, with coloured plates and wood engravings, ¿vu, ISs.

TABLE OF PHYSICAL EXAMINATION OF THE LUNGS : with Note on International Numenclatute of Physical Signs (reprinted from SiR R. D. Powell's " Diseases of the Lungs"). On one sheet, 6d.

D'ARCY POWER, M.A., M.B. OXON., F.R.c.s. ENG.

Assistant Surgeon at St. Bartholomev's Hospital; Surgeon to the Victoria Hospital for Chilulren, Chelsea; Examiner in the University of Durham; Member of the Conjoint Examining Boaid of the Royal College of Physicians (Lond.) and of Surgeons (Eng.).

THE SURGICAL DISEASES OF CHILDREN AND THEIR Treatment by Modern Methods. With Illustrations, crown 8vo, ros. $6 \mathrm{~d}$.

[Lewis's Practical Series].

URBAN PRITCHARD, M.D. EDIN., F.R.C.s. ENG.

Professor of A ural Surgery at King's College, London; Aural Surgeon to King's College Hospital; Senior Surgeon to the Royal Ear Hospital.

HANDBOOK OF DISEASES OF THE EAR FOR THE USE of S.udents and Practitioners. Fourth Edition, with Illustrations, crown 8vo.

[Lewis's Practical Series]. [In preparation.

DR. THEODOR PUSCHMANN.

Public Professor in Ordinary at the Unversity of Vienna.

A HISTORY OF MEDICAL EDUCATION FROM THE MOST Remote to the Most Recent Times. Translated and edited by Evan H. HARE, M.A. Oxon., F.R.C.S. Eng., L.S.A. Demy 8vo, 2 Is.

C. H. RALFE, M.A., M.D. CAN'TAB., F.R.C.P. LOND. Assistant Physician to the London Hospital; Examiner in Medicine to the University of Durham, \&c., \&c.

A PRACTICAL TREATISE ON DISEASES OF THE KIDneys and,Urinary Derangements. With Illustrations, crown $8 \mathrm{vo}$, Ios. $6 \mathrm{~d}$.

[Lewis's Practical Series]. 
LOUIS BATHE RAWLING, M.в., B.c. (CANT.), F.R.c.s. (ENG.). Assistant Surgeon, and Senior Demonstrator of Anatomy, St. Bartholomew's Hospitat ; Assistant Surgeon to the German Hospital, Dalston; Hunterian Professor, Royal College of Surgeons, England.

LANDMARKS AND SURFACE MARKINGS OF THE HUMAN Body. Demy 8vo, 24 plates ( 3 in colour) 5 s. nett.

[Fust published.

F. R. COWPER REED, M.A., r.g.s.

[See Cambridge Geological Series, page 5.

H. A. REEVES, F.R.c.s. EDIN.

Senior Assistant Surgeon and Teacher of Practical Surgery at the London Hospital; Surgeon to the Royal Orthopadic Hospital.

BODILY DEFORMITIES AND THEIR TREATMENT: A Handbook of Practical Orthopædics. With Illustrations, crown 8vo, 8s. 6d.

[Lewis's Practical Series.]

A. B. RENDLE, M.A. (CANTab.), B.sc. (Lond.).

[See Cambridge Biological Series, page 5.

SIDNEY H. REYNOLDS, M.A.

[See Cambridge Biological Series, page 5.

SAMUEL RIDEAL, D.Sc. LOND., F.I.C., F.c.s.

Fellow of University College, London.

I.

PRACTICAL ORGANIC CHEMISTRY; The Detection and Properties of some of the more important Organic Compounds. Second edition, r $2 \mathrm{mo}, 2 \mathrm{~s} .6 \mathrm{~d}$.

PRACTICAL CHEMISTRY FOR MEDICAL STUDENTS, required at the First Examination of the Conjoint Examining Board in England. Fcap 8vo, 2s.

J. JAMES RIDGE, M.D. Medical Officer of Health, Enfield.

ALCOHOL AND PUBLIC HEALTH. Second Edition, crown 8vo, 2s.

E. A. RIDSDALE.

Associate of the Royal School of Mines.

COSMIC EVOLUTION; being Speculations on the Origin of our Environment. Fcap. 8vo, 3s. 
SYDNEY RINGER, M.D., F.R.S.

Holme Prufessor of Clinical Medicine in University College; Physician to University College Hospital, AND

HARRINGTON SAINSBURY, M.D., F.R.c.P.

Physitian to the Royal Free Hospital and the City of Lordon Hospital for Diseases of the Chest. Victoria Park.

A HANDBOOK OF THERAPEUTICS. Thirteenth Edition, thoroughly revised, 8 vo, $\mathbf{1 6 s .}$

SYDNEY RINGER, M.D., F.R.s.

ON THE TEMPERATURE OF THE BODY AS A MEANS of Diagnosis and Prognosis in Phthisis. Second Edition, small 8vo, 2s. 6d.

R. LAWTON ROBERTS, M.D. LOND., D.P.H. CAMB., M.R.C.S. ENG. Honorary Life Nember of, and Lecturer and Examiner to, the St. Fohn Ambulance Association; F.P. for County of Denbigh.

ILLUSTRATED LECTURES ON AMBULANCE WORK.

Fifth Edition, copiously Illustrated, crown 8vo, 2s. $6 \mathrm{~d}$.

ILLUSTRATED LECTURES ON NURSING AND HYGIENE. Third Edition, with Illustrations, crown $8 \mathrm{vo}, 2 \mathrm{~s}$. 6d.

FREDERICK T. ROBERTS, M.D., B.sc., F.R.c.P.

Professor of the Principles and Practice of Medicine in University College; Physiczan to University College Hospital; Consulting Physician to Brompton Consumption Hospital, \& c.

THE THEORY AND PRACTICE OF MEDICINE. Tenth Edition, with Illustrations, large 8vo. [In the press.

H. D. ROLLESTON, M.D., F.R.c.P.

[See Cambridge Biological Series, page 5.

ROBSON ROOSE, M.D., LL.D., F.C.S.

Fellow of the Royal College of Physicians in Edinburgh, sc.

LEPROSY AND ITS PREVENTION : as Illustrated by Norwegian Experience. Crown 8vo, 3s. 6d.

WILLIAM ROSE, M.B., B.s. LOND., F.R.c.s.

Professor of Surgery in King's College, London, and Surgeon to King's College Hospital.

HARELIP AND CLEFT PALATE. With Illustrations, demy 8vo, 6s. 
BERNARD ROTH, F.R.c.s.

Orthopadic Surgeon to the Royal Alexandra Children's Hospital, Brighton, Ec.

THE TREATMENT OF LATERAL CURVATURE OF THE Spine: with Appendix giving an Analysis of rooo Consecutive Cases treated by "Posture and Exercise" exclusively (without Mechanical Support). Second edition, with Photographic and other Illustrations, royal 8vo, 1os. $6 \mathrm{~d}$.

A. RUSSELL, M.A., M.I.E.E.

[See Cambridge Physical Series, page 6.

\section{PROF. E. RUTHERFORD.}

[See Cambridge Physical Series, page 6.

JOHN SAVORY.

Member of the Society of Apothecaries, London.

A COMPENDIUM OF DOMESTIC MEDICINE AND COMpanion to the Medicine Chest: Intended as a source of easy reference tor Clergymen, Master Mariners, and Travellers; and for Families resident at a distance from professional assistance. Tenth Edition, sm. 8vo, $5 \mathrm{~s}$.

DR. B. S. SCHULTZE.

Professor of Gynecology; Director of the Lying-in Hospital, and of the Gynecological Clinic at fena.

THE PATHOLOGY AND TREATMENT OF DISPLACEments of the Uterus. Translated by J. J. MACAN, M.A., M.R.C.S., and edited by A. V. Macan, M.B., M.Ch., Master of the Rotunda Lying-in Hospital, Dublin. With I2o Illustrations, medium 8vo, I2s. $6 \mathrm{~d}$.

A. C. SEWARD, M.A., F.g.s.

[See Cambridge Biological Series, page 5.

A. E. SHIPLEY, M.A.,

AND

E. W. MACBRIDE, M.A.

[See Cambridge Biological Series, page 5 .

G. E. SHUTTLEWORTH, в.A., м.D.

Medical Examiner of Defective Children, School Board for London; late Medical Superintendent, Royal Albert Asylum for Idiots and Imbeciles of the Northern Counties, Lancaster, \&c.

MENTALLY-DEFICIENT CHILDREN : THEIR TREATMENT and Iraining. Second edition, with Illustrations, crown 8 vo, 5s. nett.

DAVID SIME, M.D.

RABIES: Its Place Amongst Germ Diseases, and its Origin in the Animal Kingdom. Royal 8vo, Ios. 6d. nett. 
FRANCIS W. SMITH, M.в., B.s.

THE SALINE WATERS OF LEAMINGTON. Second Edition, with Illustrations, crown $8 \mathrm{vo}$, is. nett.

\section{J. LEWIS SMITH, M.D.}

Physician to the New York Foundling Asylum; Clinical Professor of Diseases of Children in Bellevue Hospital Medical College.

A TREATISE ON THE MEDICAL AND SURGICAL DISeases of Infancy and Childhood. Eighth Edition, with 273 Illustrations and four plates, large $8 \mathrm{vo}, 2 \mathrm{Is}$.

E. HUGH SNELL, M.D., B.Sc. LOND.

Diplomate in Public Health of the University of Cambridge; Medical Officer of Health to the City of Coventry; late London County Council Medical Officer to the Blackwall Tunnel.

COMPRESSED AIR ILLNESS, OR SO-CALLED CAISSON

Disease. With Illustrations, demy $8 \mathrm{vo}$, ros. $6 \mathrm{~d}$.

JOHN KENT SPENDER, M.D. LOND.

Physician to the Royal Mineral Water Hospital, Bath.

THE EARLY SYMPTOMS AND THE EARLY TREATMENT of OSTEO-ARTHRITIS, commonly called Rheumatoid Arthritis, with special reference to the Bath Thermal Waters. Sm. 8vo, 2s. $6 \mathrm{~d}$.

\section{LOUIS STARR, M.D.}

Physician to the Children's Hospital, Philadelphia, \&e.

HYGIENE OF THE NURSERY. Including the General Regimen and Feeding of Infants and Children; Massage, and the Domestic Management of the Ordinary Emergencies of Early Life. Sixth Edition; with Illustrations, crown 8 vo, 3s. $6 \mathrm{~d}$.

JOHN LINDSAY STEVEN, M.D. Assistant Physician and Pathologist, Glasgow Royal Infirmary ; Physician for Out-patients, Royal Hospital for Sick Children, Glasgow, \&c.

THE PATHOIOGY OF MEDIASTINAL TUMOURS. With special reference to Diagnosis. With Plates, 8vo, 4s. 6d. 
JUKES DE STYRAP, M.R.C.P.I., ETC.

Physician-Extraordinary, late Physician in Ordinary, to the Salop Infirmary ; Consultung Physician to the South Salop and Montgomeryshire Infirmaries, etc.

I.

THE YOUNG PRACTITIONER: WITH PRACTICAL HINTS and Instructive Suggestions, as Subsidiary Aids, for his Guidance on Entering into Private Practice. Demy 8vo, 7s. 6d. nett.

II.

A CODE OE MEDICAL ETHICS: WITH GENERAL AND Special Rules for the Guidance of the Faculty and the Public in the Complex Relations of Professional Life. Fourth Edition, demy 8vo, 3s. 6d. nett.

III.

MEDICO-CHIRURGICAL TARIFES. Fifth Edition, revised and enlarged, fcap. 4to, 2s. nett.

IV.

THE YOUNG PRACTITIONER: HIS CODE AND TARIFF. Being the above three works in one volume. Demy 8 vo, ros. 6d. nett.

C. W. SUCKLING, M.D. LoND., M.R.c.P.

Professor of Materia Medica and Therapeutics at the Queen's College, Physician to the Queen's Hospital, Birmingham, etc.

I.

ON THE DIAGNOSIS OF DISEASES OF THE BRAIN Spinal Cord, and Nerves. With Illustrations, crown 8 vo, 8s. $6 \mathrm{~d}$.

II.

ON THE TREATMENT OF DISEASES OF THE NERVOUS System. Crown 8vo, 7s. 6d.

J. BLAND-SUTTON, F.R.c.s.

Assistant Surgeon to the Middlesex Hospital; Examiner in Anatomy for the Fellowship to the Royal College of Surgeons, England.

LIGAMENTS: THEIR NATURE AND MORPHOLOGY.

Third Edition, with numerous Illustrations, post 8 vo, 4 s. 6d. [Now ready.
[Ne

HENRY R. SWANZY, A.M., M.B., F.R.C.S.I.

Surgeon to the Royal Victoria Eye and Ear Hospital, and Ophthalmic Surgeon to the Adelaide Hospital, Dublin; Ex-President of the Ophthalmological Society of the United Kingdom.

A HANDBOOK OF THE DISEASES OF THE EYE AND their Treatment. Eighth Edition, illustrated with 168 wood-engravings, colour tests, \&c., small 8 vo, $12 \mathrm{~s}$. 6 d.

[Now ready. 


\title{
ALBERT TAYLOR.
}

Member Royal Sanitary Institute; late Demonstrator to the Students of the Sanitary Institute; Sanitary Inspector, City of Westminster; Late Chief Sanitary Inspector to the Vestry of St. George, Hanover Square, \&c.

THE SANITARY INSPECTOR'S HANDBOOK. Fourth Edition, with Illustrations, crown $8 \mathrm{vo}, 6 \mathrm{~s}$.

[Fust published.

\author{
H. COUPLAND TAYLOR, M.D. \\ Fellow of the Royal Meteorological Society.
}

WANDERINGS IN SEARCH OF HEALTH, OR MEDICAL and Meteorological Notes on Various Foreign Health Resorts. With Illustrations, crown $8 \mathrm{vo}, 6 \mathrm{~s}$.

JOHN W. TAYLOR, F.R.C.S. ENG.

Professor of Gynacology in the University of Birmingham; Sellior In-patient Surgeon to the Birmingham ard Midland Hospital for Women; Consulting Surgeon to the Wolverhampton Hospital for Women; Consulting Gynacological Surgeon to the Birmingham Skin and Lock Hospital, \&c.

EXTRA-UTERINE PREGNANCY. A Clinical and Operative

Study. With Illustrations, demy 8 vo, 7 s. $6 \mathrm{~d}$.

J. J. THOMSON, D.sc., LL.D., F.R.s.

[See Cambridge Physical Series, page 6.

J. C. THOROWGOOD, M.D.

Assistant Physician to the City of London Hospital for Diseases of the Chest.

THE CLIMATIC TREATMENT OF CONSUMPTION AND Chronic Lung Diseases. Third Edition, post 8vo, 3s. $6 \mathrm{~d}$.

HERBERT TILLEY, M.D., B.s. LOND., F.R.C.s. ENG.

Surgeon to the Throat Hospital, Golden Square; Lecturer on Diseases of the Nose and Throat, London Post-Graduate College and Polyclinic.

PURULENT NASAL DISCHARGES, their Diagnosis and Treatment. Second Edition, with Illustrations, crown 8vo, 4 s. mett.

M. TUCHMANN, M.R.c.s. ENG., M.D. wURzBURg. Formerly Surgeon to Out.Patients, German Hospital, Dalston.

THE EXPLORATION OF THE URETHRA AND BLADDER. With 26 Illustrations, demy 8 vo, 5 s.

A. DUNBAR WALKER, м.D., с.м.

THE PARENT'S MEDICAL NOTE BOOK. Oblong post 8vo, cloth, x8. 6d.

E. W. AINLEY WALKER, M.A., D.M. (OXON.).

Fellow and Praelector of University College, Oxford; Late Gordon Lecturer in Experimental Pathology at Guy's Hospital; formerly Radcliffe Travelling Fellow in the University of Oxford, Ec.

THE GENERAL PATHOLOGY OF INFLAMMATION, INfection, and Fever, being the Gordon Lectures for 1902 . Crown 8vo, $4 \mathrm{~s}$. 6d. nett. 
A. J. WALL, M.D. LOND.

Fellow of the Royal College of Surgeons of England; of the Medical Staff of H. M. Indian Armv (Retired List).

ASIATIC CHOLERA: its History, Pathology, and Modorn Treatment. Demy 8 vo, $6 \mathrm{~s}$.

H. MARSHALL WARD, Sc.D., F.R.s.

[See Cambridge Biological Series, page 5.

W. SPENCER WATSON, B.M. LOND., F.R.c.s. ENG.

Surgean to the Throat Department of the Great Northern Hospizal; Senior Surgeon to the Royat South London Ophthalmic Hospital.

DISEASES OF THE NOSE AND ITS ACCESSORY CAVITIES. Second Edition, with Illustrations, demy 8vo, 12s. 6d.

II.

THE ANATOMY AND DISEASES OF THE IACHRYMAL Passages. With Illustrations, demy 8vo, 2s. $6 \mathrm{~d}$.

III.

EYEBALL-TENSION : Its Effects on the Sight and its Treatment. With woodcuts, post 8 vo, $2 \mathrm{~s}$. $6 \mathrm{~d}$.

W. WYNN WESTCOTT, м.в.

H.M.'s Coroner for North-East London.

SUICIDE; its History, Literature, Jurisprudence, and Prevention. Crown 8 vo, $6 \mathrm{~s}$.

FRANK J. WETHERED, M.D.

Medical Registrar to the Middlesex Hospital, and Demonstrator of Practical Medicine in the Middleses Hospital Medical School; late Assistant Physician to the City of London Chest Hospital, Victoria Park.

MEDICAL MICROSCOPY. A Guide to the Use of the Microscope in Medical Practice. With Illustrations, crown 8vo, gs.

[Lewis's Practical Series].

W. C. D. WHETHAM, м.A.

[See Cambridge Physical Series, page 6.

L. R. WILBERFORCE, м.A.

T. C. FITZPAPARICK, M.A.

Demonstrators at the Cavendish Laboratory, Cambridge.

A LABORATORY NOTE-BOOK OF ELEMENTARY PRACtical Physics. I. Mechanics and Hrdrostatics. II. Heat and Optics. III. Magnetism axd Electricity. Quarto, paper covers, Is. each. 
SIR JOHN WILLIAMS, BART., M.D., F.R.c.P.

Consulting Physician to University College Hospital; Physician Accoucheur to H.R.H.

CANCER OF THE UTERUS: Being the Harveian Lectures for x886. Illustrated with Lithographic Plates, royal 8vo, ros. 6d.

W. WILLIAMS, M.A., M.D., D.P.H. (OXON.).

Medical Officer of Health to the Glamorgan County Council; Lecturer in Public Health to the University Colleye of South Wales and Monmouthshire, Cardiff; Examnes in

State Medicine to the Unversity of London, E.c.

DEATHS IN CHILDBED: A Preventable Mortality, being the Milroy Lectures for $590_{4}$. Demy $8 \mathrm{vo}, 2 \mathrm{~s}$. 6d. nett.

R. T. WILLIAMSON, M.D. LOND., F.R.c.P.

Medical Registrar, Royal Infirmary, and A ssistant in Medicine, Owens College, Manchester.

ON THE RELATION OF DISEASES OF THE SPINAL CORD to the Distribution and Lesions of the Spinal Blood Vessels. Royal 8vo, 2s.

J. C. WILLIS, M.A.

[See Cambridge Biological Series, page 5.

E. T. WILSON, M.B. OXON., F.R.C.P. LOND.

Physician to the Cheltenham General Hospital; Associate Metropolitan Association of Medical Officers of Health.

DISINFECTANTS AND ANTISEPTICS: HOW TO USE THEM. 4oth Thousand. In Packets of one doz. price is., by post is. xd.

¡Thoroughly revised.

BERTRAM C. A. WINDLE, F.R.s., sc.D., M.D., M.A. DUBL.

Professor of Anatomy in the University of Birmingham; sometime Examiner in Anatomy in the Universities of Cambridge, A berdeen, and Durham.

A HANDBOOK OF SURFACE ANATOMY AND LANDMARKS. Third Edition, Illustrated with plain and coloured figures, post $8 \mathrm{vo}, 4 \mathrm{~s}$. nett.

[Now ready.

EDWARD WOAKES, M.D. LOND.

Senior A ural Surgeon, London Hospital; Lecturer on Diseases of the Ear, London Hospital Medical College.

ON DEAFNESS, GIDDINESS AND NOISES IN THE HEAD.

Fourth Edition, Part i., with Illustrations, 8vo, Ios. 6 d. 
HENRY WOODS, B.A., F.G.S.

[See Cambridge Biological Series, page 5 .

\title{
A. S. WOODWARD, M.A.
}

[See Cambridge Biological Series, page 5.

\author{
OSWALD ZIEMSSEN, M.D. \\ Knight of the Iron Cross, and of the Prussian Order of the Crown.
}

\section{THE TREATMENT OF CONSTITUTIONAL SYPHILIS. Post 8vo, 3s. $6 \mathrm{~d}$.}

Lewis's Diet Charts.

Price 5s. per packet of roo charts (assorted) post free.

A suggestive set of Diet Tables for the use of Physicians, for handing to patients afterconsultation, modified to suit individual requirements, for Albuminuria, Anæmia and Debility, Constipation, Diabetes, Diarrhcea, Dyspepsia, Eczema, Fevers, Gall Stones, Gout and Gravel, Heart Disease (chronic), Nervous Diseases, Obesity, Phthisis, Rbeumatism (chronic), and Blank Cbart for otber diseases.

A special leaflet on the Diet and Management of Infants is sold separately, price 7s. 6d. per roo, or rs. per dozen, post free.

Lewis's Handy Temperature Chart.

25s. per rooo, $\mathrm{r}_{4} \mathrm{~s}$. per $500,3 \mathrm{~s} .6 \mathrm{~d}$. per $100,2 \mathrm{~s}$. per 50 , is. per 20 , carriage free. Arranged for three weeks, and specially ruled on back for recording observations on Urine.

\section{Lewis's Four-Hour Temperature Chart.}

25s. per 1000, I4s. per 500, 3s. $6 \mathrm{~d}$. per 100, $2 \mathrm{~s}$. per 50, Is. per 20, carriage free.

This form has been drawn up to meet the requirements of a chart on which the temperature and other observations can be recorded at intervals of four hours. They will be found most convenient in hospital and private practice. Each chart will last a week.

\section{Lewis's Blood Pressure and Pulse Chart.}

25s. per I000; I4s. per 500; 3s. 6d. per 100; 2s. per 50; Is. per 20, carriage free.

Clinical Chart for Temperature Observations, etc.

Arranged by W. RIGDEN, M.R.C.S. 50s. per 1000, 28s. per 500, I5s. per 250, $7 \mathrm{~s}$. per 100, or Is. per dozen, carriage free.

Each Chart is arranged for four weeks, and is ruled at the back for making notes of Cases. They are convenient in size, and are suitable botb for hospital and private practice.

Lewis's Nursing Chart.

25s. per 1000 , 14 s. per 500,3 s. 6 d. per 100, 2s. per 5o, or Is. per 20, post free.

This Chart affords a ready method of recording the progress of the case from day to day. Printed on both sides.

*** Boards to hold either of the above Charts, price is.

Lewis's Clinical Chart, specially designed for use with the Visiting

List. This Temperature Chart is arranged for four weeks and measures $6 \times 3$

inches. 20s. per 1000, IIs. 6d. per 500, 2S. 6d. per 100, 6d. per dozen, post free.

\section{Chart for Recording the Examination of Urine.}

40s. per IO00; 25s. per 500; I5s. per $250 ; 7 \mathrm{~s} .6 \mathrm{~d}$. per I00; Is. per Io.

This Chart is designed for the use of Medical Men, Analysts, and others making examinations of the Urine of patients and affords a very ready and convenient method of recording the results of the examination. 


\section{LEWIS'S PRACTICAL SERIES.}

Under this title a Series of Monographs is published, embracing the various branches of Medicine and Surgery. The volumes are written by well known Hospital Physicians and Surgeons, recognised as authorities in the subjects of which they treat. The works are of a THOROUGHLY PRACTICAL nature, calculated to meet the requirements of the practitioner and student and to present the most recent information in a compact form.

A FANDBOOK OF BACTERIOLOGICAL DIAGNOSIS FOR PRACTYTIONERS (including Instructions for the Clinical Examination of the Blood). By W. D'ESTE EMEKY, M.D., B.Sc. Lond., Assistant Bacteriologist to the Laboratories of the Royal Colleges of Physicians and Surgeons, London, \&c. With 2 Coloured Plates and 33 Illustrations, crown $8 v 0,5 s .6 \mathrm{~d}$.

DISEASES OF THE NERYOUS SYSTEM: A Handbook for Students and Practitioners. By CHARLES E. BEEVOR, M.D., LonD., F.R.C.P., Physician to the National Hospital for the Paralysed and Epileptic, the Great Northern Central Hospital, and the National Orthopædic Hospital. With lllustrations, crown 8vo, Ios. $6 \mathrm{~d}$.

THE TREATMENT OF PULMONARY CONSUMPTION. BY VINCENT D. HARRIS, M.D. LOND., F.R.C.P., Physician to the City of London Hospital for Diseases of the Chest, Victoria Park, \&c., and E. CLIFFORD BEALE, M.A., M.B. CANTAB., F.R.C.P., Physician to the City of London Hospital for Diseases of the Chest, Victoria Park, \&c. Crown 8vo, 1os. 6d.

THE SURGICAL DISEASES OF CHILDREN AND THEIR TREATMENT BY Modern Methods. By D'ARCY POWER, M.A., M.B. Oxon., F.R.C.S. Eng., Assistant Surgeon at St. Bartholomew's Hospital, \&c. With Illustrations, crown 8vo, 10s. $6 \mathrm{~d}$.

DISEASES OF THE NOSE AND THROAT. By F. de HAVILLAND HALL, M.D., F.R.C.P Lond., Physician to the Westminster Hospital, and HERBERT TILLEY, M.D., B.S. Lond., F.R.C.S. Eng., Surgeon to the Throat Hospital, Golden Square, \&c. Second edition, with two coloured plates and 80 illustrations, crown 8 vo, Ios. $6 \mathrm{~d}$.

PUBLIC HEACTH LABORATORY WORK. BY HENRY R. KENWOOD, M.B., D.P.H., F.C.S., Professor of Hygiene and Public Health, University College, \&c. Third Edition. (Part VII. contributed by W. G. SAVAGE, M.D., \&c.). With lllustrations, cr. 8vo, 1os. 6d.

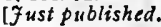

MEDICAL MICROSCOPY: A Guide to the use of the Microscope in Medical Practice. By FRANK J. WETHERED, M.D., M.R.C.P., Demonstrator of Practical Medicine in the Middlesex Hospital Medical School, \&c. With Illustrations, crown 8vo, gs.

MEDICAL ELECTRICITY: A Practical Handbook for Students and Praotitioners. By H. LEWIS JONES, M.A., M.D., F.R.C.P. Fourth edition, with 168 lllustrations, demy 8vo, i2s $6 \mathrm{~d}$. nett.

[fust published.

HYGIENE AND PUBLIC HEALTH. By LOUIS PARKES, M.D. D.P.H. LoND, UNIV, Fellow of the Sanitary Institute, and HENRY R. KENWOOD, M.B., D.P.H., F.C S., Professor of Hygiene and Public Health, University College. Second Edition, with 88 Illustrations, cr. 8vo, I2s.

MANUAL OF OPHTHALMIC PRACTICE. By C. HIGGENS, F.R.C.S., Ophthalmic Surgeon to Guy's Hospital; Lecturer on Ophthalmology at Guy's Hospital Medical School. Second Edition, revised and edited by A. W. ORMOND, F.R.C.S.E., \&c. With lllustrations, cr. 8 vo, $7 \mathrm{~s} .6 \mathrm{~d}$.

[Fust published.

A PRACTICAL TEXTBOOK OF THE DISEASES OF WOMEN. BY ARTHUR H. N. LEWERS, M.D. Lond., F.R.C.H. Lond., Senicr Obstetric Physician to the London Hospital, etc. Sixth Edition, with Illustrations, crown 8vo, ros. 6d. [Fust published.
[Fu

ANASTHETICS: Their Uses and Administration. By DUDLEY W. BUXTON, M.D., B.S., M.R.C.P., Administrator of Anæsthetics and Lecturer in University College Hospital, \&c. Fourth Edition, with Illustrations, crown 8 vo.

In the press.

ON FEYERS: Their History, Etiology, Diagnosis, Prognosis, and Treatment. By ALEXANDER COLLIE, M.D. A berd., M.R.C.P. Illustrated with Coloured Plates, crown 8 vo, 8s. 6d.

HANDBOOK OF DISEASES OF THE EAR: For the Use of Students and Practitioners. By URBAN PKITCHAKD, M.D. Edin., F.K.C.S. Eng., Protessor of Aural Surgery at King's College, London. Fourth Edition, with lllustrations, crown 8vo. [In proparation.

A PRACTICAL TREATISE ON DISEASES OF THE KIDNEYS AND Urinary Derangements. By C. H. RALFE, M.A., M.D., F.R.C.P., Assistant Physician to the London Hospital, \&c. With Illustrations, cr. 8vo, ros. 6d.

DENTAL SURGERY FOR MEDICAL PRACTYTIONERS AND STUDENTS of Medicine. By ASHLEY W. BARKETT, M.B. Lond., M.K.C.S., L.D.S., Consulting Dental Surgeon to the London Hospital. Fourth edition, with 1llustrations, cr, 8vo, 3s. $6 \mathrm{~d}$.

[Fust published.

PRACTICAL DEFORMYTIES AND THEIR TREATMENT. A Handbook of Practical Urthopedics. By H. A. REEVES, F.R.C.S. Edin., Senior Assistant Surgeon at the London Hospital, etc. With 1llustrations, cr. 8vo, 8s. 6d. 


\title{
THE NEW SYDENHAM SOCIETYYS PUBLICATIONS.
}

\author{
President:-W. OsLer, M.D., LL.D., F.R.S. \\ Honorary Secretary:-Jonathan Hutchinson, EsQ., F.R.S. \\ Treasurer:-Henry Power, EșQ., F.R.C.S. - \\ $\therefore$ \\ ANNUAL SUBSCRIPTION, ONE GUINEA.
}

The Society is now issuing an important "Atlas of Clinical Medicine, Surgery, and Pathology," designed to form a pictorial guide to diagnosis. A volume of Selected Monographs, or a translation of some important Continental work, will be issued from time to time.

Amongst the works issued are Limbeck's "Pathology of the Blood," Helferich "On Fractures and Dislocations," Laveran's "Paludism," Pozzi's "Gynecology," Spiegelberg's "Midwifery," works by Charcot, Duchenne, Begbie, Billroth, Graves, Koch, Hebra, Guttmann, and a valuable and exbaustive "Lexicon of Medicine and the Allied Sciences," just completed.

The Annual Report, with full list of works published, prospectus of the new Atlas of Clinical Illustrations of Medicine and Pathology, and all further information will be sent on application.

\section{PERIODICAL WORKS PUBLISHED BY H. K. LEWIS.}

THE BRITISH JOURNAL OF DERMATOLOGY. Under the direction of H. G. Brooke, H. Radcliffe-Crocker, T. Colcott Fox, J. Galloway, E. Graham Little, Sir Stephen Mackenzie, Malcolm Morris, J. J. Pringle, J. H. Sequeira, A. Shillitoe, E. Stainer, J. Herbert Stowers, Arthur Whitfield; Edited by J. M. H. MacLeod. Published monthly, xs. Annual Subscription, 12s. post free.

THE GLASGOW MEDICAL JOURNAL. Edited by John Lindsay Steven, M.D., and T. K. Monro, M.D. Published Monthly. Annual Subscription, 2os., post free. Single numbers, 2s. each.

LIVERPOOL MEDICO-CHIRURGICAL JOURNAL, including the Proceedings of the Liverpool Medical Institution. Published twice yearly, 3s. 6d. each number.

TRANSACTIONS OF THE DERMATOLOGICAL SOCIETY OF GREAT BRITAIN AND IRELAND. Vols. I. to X., royal 8vo, 5s. each.

NORTH LONDON OR UNIVERSITY COLLEGE HOSPITAL REPORTS, SURGICAL CASES. 190r, 8vo, 3s. 6d. nett.

THE THERAPEUTIC GAZETTE. A Monthly Journal, devoted to the Science of Pharmacology, and to the introduction of New Therapeutic Agents. Edited by Dr. H. A. Hare and Dr. E. Martin. Annual Subscription, ros., post free.

MIDDLESEX HOSPITAL, REPORTS OF THE MEDICAL, SURGICAL, AND PATHOLOGICAL REGISTRARS. Demy 8vo, 2s. 6d, nett. each volume.

${ }_{*}^{*}$ MR. LEwis is in constant communication with the leading publishing firms in America, and has transactions with them for the sale of his publications in that country. Advantageous arrangements are made in the interests of Authors for the publishing of their works in the United States.

Mr. Lewis's publications can be procured of all Booksellers in any part of the world. 
